\title{
Hyper-Distorted Tungsten Allyl Complexes and their Stereoselective \\ Deprotonation to Form Dihapto-Coordinated Dienes
}

Daniel P. Harrison, Adam C. Nichols-Nielander, Victor E. Zottig, Laura Strausberg, Rebecca J. Salomon, Carl O. Trindle, Michal Sabat, ${ }^{\%}$ T. Brent Gunnoe, Diana A. Iovan, "William H. Myers, ${ }^{\#}$ and W. Dean Harman $^{*}$

Department of Chemistry, University of Virginia, Charlottesville, Virginia 22904, and \#Department of Chemistry, University of Richmond, Richmond, Virginia 27173

${ }^{\%}$ Nanoscale Materials Characterization Facility Department of Materials Science and Engineering University of Virginia, Charlottesville, VA 22904

E-mail: wdh5z@virginia.edu

Table of Contents

\begin{tabular}{|c|c|}
\hline S1-S2 & Supplemental Information Title Page, Table of Contents \\
\hline S2 & Figure S1, Acronyms \\
\hline S4-S16 & Experimental Details \\
\hline S4 & {$\left[\mathrm{TpW}(\mathrm{NO})\left(\mathrm{PMe}_{3}\right)\left(2,3-\eta^{2}\right.\right.$-cyclohexan-2-en-1-ylium)][OTf]. 3.} \\
\hline S5 & $\mathrm{TpW}(\mathrm{NO})\left(\mathrm{PMe}_{3}\right)\left(1,2-\eta^{2}\right.$-cyclohexa-1,3-diene). 4p, 4d. \\
\hline S5 & Single isomer synthesis of $\mathbf{4 d}$. \\
\hline S6 & $\mathrm{TpW}(\mathrm{NO})\left(\mathrm{PMe}_{3}\right)\left(3,4-\eta^{2}-(1-(\right.$ pyridin-1(2H)-yl)ethanone)). 7d. \\
\hline S7 & {$\left[\mathrm{TpW}(\mathrm{NO})\left(\mathrm{PMe}_{3}\right)\left(2,3-\eta^{2}-(\right.\right.$ cyclohex-2-en-1-yltrimethylphosphonium)](OTf). 8c. } \\
\hline S7 & {$\left[\mathrm{TpW}(\mathrm{NO})\left(\mathrm{PMe}_{3}\right)\left(2,3-\eta^{2}\right.\right.$-cyclopent-2-en-ylium)][OTf]. 9.} \\
\hline S8 & $\mathrm{TpW}(\mathrm{NO})\left(\mathrm{PMe}_{3}\right)\left(1,2-\eta^{2}\right.$-cyclopenta-1,3-diene): 10p, 10d. \\
\hline S9 & {$\left[\mathrm{TpW}(\mathrm{NO})\left(\mathrm{PMe}_{3}\right)\left(2,3-\eta^{2}\right.\right.$-propan-1-ylium)][OTf]. exo-11, endo-11. } \\
\hline S10 & TpW(NO)(PMe $)\left(1,2-\eta^{2}\right.$-(2,3-dimethylbut-3-en-2-ylium)][OTf]. exo-12, endo-12. \\
\hline S11 & $\mathrm{TpW}(\mathrm{NO})\left(\mathrm{PMe}_{3}\right)\left(5,6-\eta^{2}-4-\right.$ methylcyclohexa-2,4-dienone). 17. \\
\hline S11 & [TpW(NO)(PMe 3$)\left(2,3-\eta^{2}\right.$-1-methyl-4-oxocyclohex-2-en-1-ylium)][OTf]. 18. \\
\hline S12 & {$\left[\mathrm{TpW}(\mathrm{NO})\left(\mathrm{PMe}_{3}\right)\left(2,3-\eta^{2}-(4-o x o c y c l o h e x-2-e n-1-y l i u m)\right][\mathrm{OTf}] .20 p\right.$.} \\
\hline S13 & {$\left[\mathrm{TpW}(\mathrm{NO})\left(\mathrm{PMe}_{3}\right)\left(2,3-\eta^{2}\right.\right.$-(4-oxocyclohex-2-en-1-ylium)][OTf]. 20d (and 20p). } \\
\hline S13 & {$\left[\mathrm{TpW}(\mathrm{NO})\left(\mathrm{PMe}_{3}\right)\left(2,3-\eta^{2}-(4-(\right.\right.$ dimethyliminio)cyclohex-2-en-1-ylium))](OTf) $2.21 \mathrm{p}$.} \\
\hline S14 & $\mathrm{TpW}(\mathrm{NO})\left(\mathrm{PMe}_{3}\right)\left(\mathrm{Exo}-1,2-\eta^{2}-2,3-\right.$ dimethylbutadiene). 22 (exo-22). \\
\hline S14 & $\mathrm{TpW}(\mathrm{NO})\left(\mathrm{PMe}_{3}\right)\left(1,2-\eta^{2}-2,3-\right.$ dimethylbutadiene). Endo-22, Exo-22. \\
\hline S15 & $\mathrm{TpW}(\mathrm{NO})\left(\mathrm{PMe}_{3}\right)\left(1,2-\eta^{2}-2,3-\right.$ dimethylbut-1-ene). endo-23, exo-23. \\
\hline S16 & $\mathrm{Na} / \mathrm{Hg}$ amalgam reduction of 12. \\
\hline S17-S33 & ${ }^{1} \mathrm{H}$ and ${ }^{13} \mathrm{C}$ NMR spectra of new compounds. \\
\hline S17 & Compound 3: ${ }^{1} \mathrm{H}$ and ${ }^{13} \mathrm{C}$ NMR $\left(\mathrm{CD}_{3} \mathrm{CN}\right)$ \\
\hline S18 & Compound $4 \mathrm{p}, 4 \mathrm{~d}:{ }^{1} \mathrm{H}$ and ${ }^{13} \mathrm{C}$ NMR $\left(\mathrm{CDCl}_{3}\right)$ \\
\hline S19 & Compound $\mathbf{4 d :}{ }^{1} \mathrm{H}$ NMR $\left(\mathrm{CDCl}_{3}\right)$ \\
\hline S20 & Compound 7d: ${ }^{1} \mathrm{H}$ and ${ }^{13} \mathrm{C} \mathrm{NMR}\left(\mathrm{CDCl}_{3}\right)$ \\
\hline
\end{tabular}




\begin{tabular}{|c|c|}
\hline S21 & Compound 8c: ${ }^{1} \mathrm{H}$ and ${ }^{13} \mathrm{C}$ NMR $\left(\mathrm{CDCl}_{3}\right)$ \\
\hline S22 & Compound 10p,10d: ${ }^{1} \mathrm{H}$ and ${ }^{13} \mathrm{C} \mathrm{NMR}\left(\mathrm{CDCl}_{3}\right)$ \\
\hline S23 & Compound Exo-11, Endo-11: ${ }^{1} \mathrm{H}$ and ${ }^{13} \mathrm{C}$ NMR $\left(\mathrm{CD}_{3} \mathrm{CN}\right)$ \\
\hline S24 & Compound Exo-12, Endo-12: ${ }^{1} \mathrm{H}$ and ${ }^{13} \mathrm{C}$ NMR $\left(\mathrm{CD}_{3} \mathrm{CN}\right)$ \\
\hline S25 & Compound 17: ${ }^{1} \mathrm{H}$ and ${ }^{13} \mathrm{C} \mathrm{NMR}\left(\mathrm{CDCl}_{3}\right)$ \\
\hline S26 & Compound 18: ${ }^{1} \mathrm{H}$ and ${ }^{13} \mathrm{C}$ NMR $\left(\mathrm{CD}_{3} \mathrm{CN}\right)$ \\
\hline S27 & Compound 20p: ${ }^{1} \mathrm{H}$ and ${ }^{13} \mathrm{C}$ NMR $\left(\mathrm{CD}_{3} \mathrm{CN}\right)$ \\
\hline S28 & Compound 20d (and 20p): ${ }^{1} \mathrm{H}$ and ${ }^{13} \mathrm{C} \mathrm{NMR}\left(\mathrm{CD}_{3} \mathrm{CN}\right)$ \\
\hline S29 & Compound 21p: ${ }^{1} \mathrm{H}$ and ${ }^{13} \mathrm{C}$ NMR $\left(\mathrm{CD}_{3} \mathrm{CN}\right)$ \\
\hline S30 & Compound Exo-22: ${ }^{1} \mathrm{H}$ and ${ }^{13} \mathrm{C}$ NMR $\left(\mathrm{CDCl}_{3}\right)$ \\
\hline S31 & Compound Exo-22, Endo-22: ${ }^{1} \mathrm{H}$ and ${ }^{13} \mathrm{C}$ NMR $\left(\mathrm{CDCl}_{3}\right)$ \\
\hline S32 & Compound Exo-23, Endo-23: ${ }^{1} \mathrm{H}$ and ${ }^{13} \mathrm{C}$ NMR $\left(\mathrm{CDCl}_{3}\right)$ \\
\hline S33 & ${ }^{1} \mathrm{H} N M R$ of $\mathrm{Na} / \mathrm{Hg}$ reduction of Exo-12, Endo-12 \\
\hline S34 & $\begin{array}{c}\text { Table S1: Calculated Structural Energy and Number of Associated Imaginary } \\
\text { Vibrational Frequencies for Compounds Numbers in Article }\end{array}$ \\
\hline S35 & $\begin{array}{l}\text { Table S2: Calculated Structural Energy and Number of Associated Imaginary } \\
\text { Vibrational Frequencies for Theoretical Tungsten Complexes }\end{array}$ \\
\hline S36 & Cartesian coordinates and Bond Connectivity for Calculated Structures \\
\hline S36 & PDB Data: Table of Contents \\
\hline S116 & Larger image of the $\mathrm{HOMO}$ of $\left\{\mathrm{TpW}(\mathrm{NO})\left(\mathrm{PMe}_{3}\right)\right\}$ \\
\hline S132-S133 & $\begin{array}{c}\text { Figure S2: Energy versus reaction coordinate diagrams for distorted allyl isomers } \\
\text { and selected transition state structures }\end{array}$ \\
\hline S134 & Supplemental Information References \\
\hline
\end{tabular}

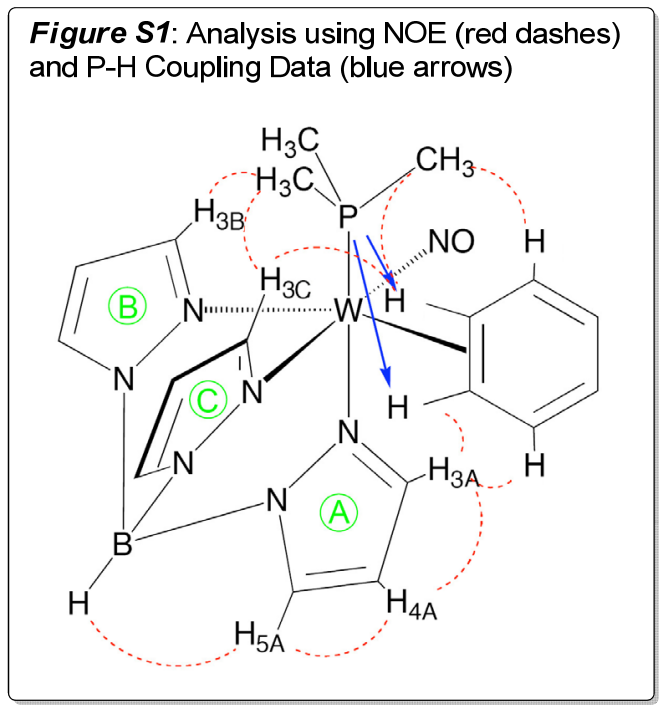

Acronyms: cd - coordination diasteriomer; cdr - coordination diasteriomer ratio; DCM dichloromethane; DME - 1,2-dimethoxyethane; MeCN - acetonitrile; DMA - N,Ndimethylacetamide; $\mathrm{Et}_{2} \mathrm{O}$ - diethyl ether; P - proximal; D - distal; CV - cyclic voltammetry; $\mathrm{NEt}_{3}$ - triethyl amine 


\section{Experimental Details.}

General Experimental Methods. NMR spectra were obtained on a 300, 500, or $600 \mathrm{MHz}$ spectrometer (Varian INOVA or Bruker Avance). All chemical shifts are reported in ppm and proton and carbon shifts are referenced to tetramethylsilane (TMS) utilizing residual ${ }^{1} \mathrm{H}$ or ${ }^{13} \mathrm{C}$ signals of the deuterated solvents as an internal standard. Phosphorus NMR signals are referenced to $85 \% \mathrm{H}_{3} \mathrm{PO}_{4}(\delta=0.00)$ using a triphenylphosphate external standard $(\delta=-16.58)$. Coupling constants $(J)$ are reported in hertz $(\mathrm{Hz})$. Infrared spectra (IR) were recorded as a glaze on a MIDAC Prospect Series (Model PRS) spectrometer fitted with a Horizontal Attenuated Total Reflectance (HATR) accessory (Pike Industries), or on a Nicolet Avatar 360 FT-IR spectrometer equipped with an ASI-DiComp diamond anvil ATR assembly. Electrochemical experiments were performed under a dinitrogen atmosphere using a BAS Epsilon EC-2000 potentiostat. Cyclic voltammetry data were aquired at ambient temperature $\left(\sim 25^{\circ} \mathrm{C}\right)$ at 100 $\mathrm{mV} / \mathrm{s}$ in a standard three-electrode cell with a glassy carbon working electrode, $N, N$ dimethylacetamide (DMA) or acetonitrile (MeCN) solvent (unless otherwise specified), and tetrabutylammonium hexafluorophosphate (TBAH) electrolyte (approx. $0.5 \mathrm{M}$ ). All potentials are reported versus NHE (Normal Hydrogen Electrode) using cobaltocenium hexafluorophosphate $\left(E_{1 / 2}=-0.78 \mathrm{~V}\right)$, ferrocene $\left(E_{1 / 2}=+0.55 \mathrm{~V}\right)$, or decamethylferrocene $\left(E_{1 / 2}=\right.$ $+0.04 \mathrm{~V}$ ) as an internal standard. The peak-to-peak separation was less than $100 \mathrm{mV}$ for all reversible couples. Elemental analyses (EA) were obtained from Atlantic Microlabs and agree to within $0.4 \%$ for $\mathrm{C}, \mathrm{H}$, and $\mathrm{N}$. High resolution electrospray ionization mass spectrometry (ESI-MS) analyses were obtained from the University of Richmond from samples dissolved in acetonitrile then mixed 3:1 with $0.1 \mathrm{M}$ aqueous sodium trifluoroacetate ( $\mathrm{NaTFA})$ using [ $\left.\mathrm{Na}(\mathrm{NaTFA})_{\mathrm{x}}\right]^{+}$ clusters as an internal standard. Data are reported for the dominant peaks in the isotopic envelope as their observed and calculated masses and their percentage abundance relative to the parent ion, followed by the difference between the observed and calculated masses in ppm, and the ion analyzed, e.g. (obs'd (\%), calc'd (\%), ppm, $\left.(M+Z)^{+}\right)$, where $Z^{+}=$proton or sodium ion. Unless otherwise noted, all synthetic reactions were performed in a glovebox under a dry nitrogen atmosphere. $\mathrm{CH}_{2} \mathrm{Cl}_{2}$ and benzene were purified by passage through a column packed with activated alumina. Other solvents and liquid reagents were thoroughly purged with dry nitrogen prior to use. Deuterated solvents were used as received from Cambridge Isotopes. Pyrazole (pz) protons of the (tris-pyrazolyl)borate, Tp, ligand were uniquely assigned using a combination of 2-dimensional NMR experiments and phosphorousproton coupling (see Figure S1 in supplemental information). ${ }^{1}$ When unambiguous assignments were not possible, pz protons were labeled as Tp protons. Coordination diastereomers are described as either proximal ( $p$ ) or distal (d) based on the proximity of a defining feature (e.g., the "carbocationic" center of an allyl ligand) to the $\mathrm{PMe}_{3}$ ligand. Synthesis of compounds $\mathrm{TpW}(\mathrm{NO})\left(\mathrm{PMe}_{3}\right)\left(\eta^{2}\right.$-benzene), ${ }^{1} 5,{ }^{2} \mathbf{7 p},{ }^{3} 9,{ }^{4} 14,{ }^{5}$ and $19^{6}$ have been previously reported. 19 can be isolated as a single isomer or a mixture of coordination diastereomers. ${ }^{6}$

DFT Calculations. Initial structures were built in Spartan $^{7}$ and optimized with the extended version of the PM3 semi-empirical method available in that package, or in GAUSSVIEW (5.0.8) with the PM6 semi-empirical method in GAUSSIAN 09. ${ }^{8} 9$ These structures were refined stepwise in Spartan and Gaussian using B3LYP and a series of basis functions incorporating LANL2 pseudopotentials and associated basis functions provided in those packages or directly 
from the PM6 structures. The most demanding calculations reported here put the LANL2DZ pseudopotential and its basis only on the $\mathrm{W}$ atom, and used the 6-31G(d) basis for all other atoms.

For transition state structures, vibrational analysis revealed the presence of a single imaginary frequency. In all other cases, vibrational analyses verified that optimized structures were located at local minima, with the presence of only real frequencies.

Many of the systems calculated herein have very soft vibrational modes. This has the consequence that in many cases reports from vibrational calculations showed small violations of the convergence criteria on the predicted root-mean-square and/or maximum displacement, for structures which had satisfied all convergence criteria in the optimization step. This unsatisfactory behavior can be remedied by reoptimization, computing the force matrix at each optimization step, and using the UltraFine grid for numerical integrations. Our spot checks showed that structures and zero point vibrational energies were unchanged by this expensive refinement. For this reason we believe that the structures, calculated energies, and zero-point energy values computed with default convergence criteria (FinGrid) for optimization, are reliable for comparisons reported here.

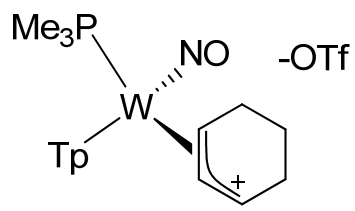

[TpW(NO)(PMe $)\left(2,3-\eta^{2}\right.$-cyclohexan-2-en-1-ylium)][OTf]. 3. 1,3-Cyclohexadiene (1.05 g, 13.1 $\mathrm{mmol}$ ) was added to an oven-dried test tube containing a heterogeneous yellow solution of $\mathrm{TpW}(\mathrm{NO})\left(\mathrm{PMe}_{3}\right)\left(\eta^{2}\right.$-benzene) $(0.525 \mathrm{~g}, 0.904 \mathrm{mmol})$ in $\mathrm{DME}(2.71 \mathrm{~g})$. The solution was added to a $60{ }^{\circ} \mathrm{C}$ oil bath and allowed to stir. Upon warming the solution became a brown-slightly purple homogeneous solution. After $1.5 \mathrm{~h}$, the solution was removed from the warm bath and allowed to cool for 5 minutes. A solution of HOTf $(0.135 \mathrm{~g}, 0.900 \mathrm{mmol})$ in MeCN $(0.695 \mathrm{~g})$ was added to the solution to make a yellow solution that precipitated a yellow solid from the shortly thereafter. After $2 \mathrm{~h} 15 \mathrm{~min}$, the yellow precipitate was collected on a $15 \mathrm{~mL}$ medium porosity fritted funnel, washed with $\sim 4 \times 0.2 \mathrm{~g}$ DME, and placed under vacuum $(0.403 \mathrm{~g}, 0.550 \mathrm{mmol}, 61 \%$ yield). ${ }^{1} \mathrm{H} N M R\left(\mathrm{CD}_{3} \mathrm{CN}, \delta\right): 8.41(\mathrm{~d}, J=2.0,1 \mathrm{H}, \mathrm{PzB} 3), 8.13(\mathrm{~d}, J=2.0,1 \mathrm{H}, \mathrm{PzA} 3), 8.03$ (d, $J=2.0$, 1H, PzB5), 7.97 (d, J = 2.0, 1H, PzC5), 7.96 (d, $J=2.0,1 \mathrm{H}, \mathrm{PzC} 3$ ), 7.81 (d, $J=2.0,1 \mathrm{H}, \mathrm{PzA} 5), 6.59$ $(\mathrm{m}, 1 \mathrm{H}, \mathrm{H} 1), 6.55(\mathrm{t}, J=2.0,1 \mathrm{H}, \mathrm{PzC} 4), 6.53(\mathrm{t}, J=2.0,1 \mathrm{H}, \mathrm{PzB} 4), 6.34(\mathrm{t}, J=2.0,1 \mathrm{H}, \mathrm{PzA} 4), 5.13$ $(\mathrm{t}, J=7.4,1 \mathrm{H}, \mathrm{H} 2), 4.38\left(\mathrm{dtt}, J=7.4,1.6,{ }^{3} J_{\mathrm{PH}}=14.5,1 \mathrm{H}, \mathrm{H} 3\right), 3.34(\mathrm{~m}, 1 \mathrm{H}, \mathrm{H} 6), 3.26(\mathrm{~m}, 2 \mathrm{H}$, $\mathrm{H}^{\prime} / \mathrm{H} 4$ ), 2.47 (dddd, J = 15.4, 10.8, 6.4, 1.4, 1H, H4'), 1.59 (m, 1H, H5), 1.33 (m, 1H, H5'), 1.20 (d, $\left.J_{\mathrm{PH}}=9.8,9 \mathrm{H}, \mathrm{PMe}_{3}\right) .{ }^{13} \mathrm{C} N M R\left(\mathrm{CD}_{3} \mathrm{CN}, \delta\right): 142.3(\mathrm{PzA} 3), 146.4(\mathrm{PzB} 3), 143.2(\mathrm{PzC} 3), 139.6(\mathrm{PzA} 5)$, 139.5 (PzB5/PzC5), 138.3 (C1), 109.5/109.0 (PzB4/PzC4), 108.1 (PzA4), 103.8 (C2, d, $\left.{ }^{2} J_{P C}=3.5\right)$, $70.0\left(\mathrm{C} 3, \mathrm{~d}, J_{\mathrm{PC}}=12.6\right), 27.1(\mathrm{C} 4), 27.0(\mathrm{C} 6), 26.9(\mathrm{C} 5), 13.4\left(\mathrm{PMe}_{3}, \mathrm{~d},{ }^{1} J_{\mathrm{PC}}=32.7\right) .{ }^{31} \mathrm{P} \mathrm{NMR}$ $\left(\mathrm{CDCl}_{3}, \delta\right):-7.85\left(J_{\mathrm{WP}}=273\right) . \mathrm{IR}: v_{\mathrm{BH}}=2522 \mathrm{~cm}^{-1}, v_{\mathrm{NO}}=1635 \mathrm{~cm}^{-1}$. CV $(\mathrm{MeCN}): E_{\mathrm{p}, \mathrm{a}}=+1.83 \mathrm{~V}, E_{\mathrm{p}, \mathrm{c}}=$ -0.95. ESI-MS: obs'd (\%), calc'd (\%), ppm (M-OTf) ${ }^{+}: 582.1675$ (89.7), 582.1672 (86.8), 0.5; 583.1697 (63.2), 583.1698 (79.3), 0.1; 584.1698 (100), 584.1695 (100), 0.5; 585.1759 (52.2), 585.1739 (40.1), 3.3; 586.1731 (100), 586.1728 (84.9), 0.5. Anal. Calc'd for $\mathrm{C}_{19} \mathrm{H}_{30} \mathrm{BF}_{3} \mathrm{~N}_{7} \mathrm{O}_{4} \mathrm{PSW}$ : C, 31.04; H, 4.11; N, 13.34. Found: C, 31.26; H, 3.90; N, 13.33. 


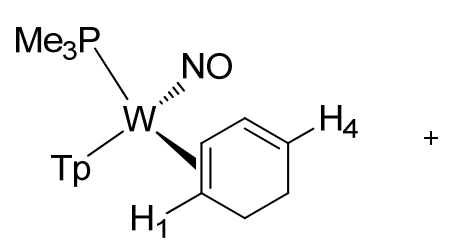

$\mathrm{P}($ roximal)

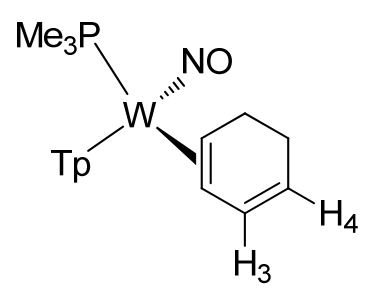

$\mathrm{D}$ (istal)

TpW(NO)(PMe $)$ (1,2- $\eta^{2}$-cyclohexa-1,3-diene). 4p, 4d. 1,3-Cyclohexadiene (0.261 g, $\left.3.3 \mathrm{mmol}\right)$ was added to a homogeneous yellow solution of $\operatorname{TpW}(\mathrm{NO})\left(\mathrm{PMe}_{3}\right)\left(\eta^{2}\right.$-benzene) $(0.151 \mathrm{~g}, 0.260$ $\mathrm{mmol})$ in DME $(3.0 \mathrm{~g})$, and allowed to stir at room temperature for 20 hours. The resulting dark brown solution was precipitated over a stirring mixture of $20 \mathrm{~mL}$ ether and $50 \mathrm{~mL}$ hexanes. The brown/purple precipitate was filtered over a $30 \mathrm{~mL}$ fine-porosity fritted funnel and discarded. The yellow filtrate was evaporated to dryness and dissolved in minimal DME. About $0.5 \mathrm{~mL}$ acetonitrile was added to the solution, which stirred ten minutes and evaporated to dryness. 1 $\mathrm{mL}$ acetonitrile was triturated with the residue; ether was added dropwise to encourage precipitation. Majority of solvent was evaporated, but did not give precipitation. Residue was dissolved in minimal DME and precipitated dropwise over $40 \mathrm{~mL}$ stirring water. A pale tan solid was filtered over a $15 \mathrm{~mL}$ fine-porosity fritted funnel and stored overnight in a desiccator to give $0.054 \mathrm{~g}\left(0.093 \mathrm{mmol}, 36 \%\right.$ yield). ${ }^{1} \mathrm{H} \mathrm{NMR}\left(\mathrm{CDCl}_{3}, \delta\right): 8.25(\mathrm{~d}, J=2.0,1 \mathrm{H}, \mathrm{PzA} 3(\mathrm{~d})), 8.08(\mathrm{~m}$, $1 \mathrm{H}, \operatorname{PzB} 3(\mathrm{p}+\mathrm{d})), 8.05(\mathrm{~d}, J=2.0,1 \mathrm{H}, \operatorname{PzA} 3(\mathrm{p})), 7.70(\mathrm{~d}, J=2.0,1 \mathrm{H}, \operatorname{PzB} 5(\mathrm{p})), 7.68(\mathrm{~d}, J=2.0,1 \mathrm{H}$, $\operatorname{PzB} 5(\mathrm{~d})), 7.61(\mathrm{~d}, J=2.0,1 \mathrm{H}, \operatorname{PzA5}(\mathrm{p})), 7.57(\mathrm{~d}, J=2.0,1 \mathrm{H}, \operatorname{PzA5}(\mathrm{d})), 7.34(\mathrm{~d}, J=2.0,1 \mathrm{H}$, PzC3(d)), $7.28(\mathrm{~d}, J=2.0,1 \mathrm{H}, \mathrm{PzC}(\mathrm{p})), 6.29(\mathrm{t}, J=2.0,1 \mathrm{H}, \mathrm{PzB} 4(\mathrm{~d})), 6.27(\mathrm{t}, J=2.0,1 \mathrm{H}, \mathrm{PzB} 4(\mathrm{p}))$, $6.21(\mathrm{t}, J=2.0,1 \mathrm{H}, \mathrm{PzA} 4(\mathrm{p})), 6.18(\mathrm{t}, J=2.0,1 \mathrm{H}, \mathrm{PzA} 4(\mathrm{~d})), 6.17 / 6.16(\mathrm{t}, J=2.0,1 \mathrm{H}, \mathrm{PzC} 4(\mathrm{p}+\mathrm{d}))$, $1.28\left(\mathrm{~d}, J=8.3,9 \mathrm{H}, \mathrm{PMe}_{3}(\mathrm{p})\right), 1.27\left(\mathrm{~d}, J=8.2,9 \mathrm{H}, \mathrm{PMe}_{3}(\mathrm{~d})\right)$, Distal Diene (d-Major isomer): 6.68 (ddd, $J=9.0,5.6,2.9,1 \mathrm{H}, \mathrm{H3}), 5.23$ (ddd, $J=9.0,6.6,2.0,1 \mathrm{H}, \mathrm{H} 4), 3.65(\mathrm{~m}, 1 \mathrm{H}, \mathrm{H} 6), 2.66(\mathrm{~m}, 1 \mathrm{H}$, H1/H6'), $2.45(m, 1 H, H 5), 1.94\left(m, 1 H, H 5^{\prime}\right), 1.68(m, 1 H, H 2)$, Proximal Diene (p-minor isomer): 6.48 (ddd, $J=9.0,4.8,2.7,1 \mathrm{H}, \mathrm{H} 3), 5.24(\mathrm{~m}, 1 \mathrm{H}, \mathrm{H} 4), 3.33(\mathrm{~m}, 1 \mathrm{H}, \mathrm{H} 6$ (anti)), 2.89 (ddd, $J=10.3$, $\left.5.0,{ }^{3} J_{\mathrm{PH}}=14.2,1 \mathrm{H}, \mathrm{H} 2\right), 2.66(\mathrm{~m}, 1 \mathrm{H}, \mathrm{H} 6(\mathrm{syn})), 2.45(\mathrm{~m}, 1 \mathrm{H}, \mathrm{H} 5), 1.95\left(\mathrm{~m}, 1 \mathrm{H}, \mathrm{H} 5{ }^{\prime}\right), 1.38(\mathrm{~d}, J=$ 10.1, 1H, H1). ${ }^{13} \mathrm{C}$ NMR $\left(\mathrm{CDCl}_{3}, \delta\right): 144.5$ (PzA3(d)), 143.4/143.3 (PzB3(p,d)), 142.1 (PzA3(p)), 140.2/140.1 (PzC3(p,d)), 136.4 (Tp5), 136.2 (Tp5), 135.6 (2 Tp5's), 135.2 (Tp5), 134.8 (Tp5), 106.3 (Tp4), 106.1 (Tp4), 105.7 (Tp4), 105.5 (3 Tp4's), 14.0 (d, ${ }^{1} J_{P C}=27.8, \mathrm{PMe}_{3}(\mathrm{p})$ ), $13.4\left(\mathrm{~d},{ }^{1} J_{\mathrm{PC}}\right.$ = 27.8, $\left.\mathrm{PMe}_{3}(\mathrm{~d})\right)$, Distal Diene: 133.4 (C3), 120.8 (C4), 56.6 (d, JPC =11.9, C1), 50.8 (C2), 23.5 (C5), 21.6 (C6), Proximal Diene: 130.8 (C3), 120.8 (C4), 55.4 (C1), 50.8 (d, JPC = 8.4, C2), 26.5 (C6), $23.5(C 5) .{ }^{31}$ P NMR: $\left(C^{2} C_{3}, \delta\right):-9.75\left(J_{W P}=286 \mathrm{~Hz}\right),-11.94\left(J_{W P}=284 \mathrm{~Hz}\right) . I R: v_{B H}=2488 \mathrm{~cm}^{-1}, v_{N O}$ $=1554 \mathrm{~cm}^{-1} . \mathrm{CV}$ (DMA): $E_{\mathrm{p}, \mathrm{a}}=+0.44 \mathrm{~V}$. ESI-MS: obs'd (\%), calc'd (\%), ppm, $(\mathrm{M}+\mathrm{H})^{+}: 594.1664$ (86.9), 594.1672 (86.2), 1.3; 595.1688 (90), 595.1698 (79.6), 1.7; 596.1687 (100), 596.1696 (100), 1.4; 597.1751 (45.4), 597.1739 (40.8), 2.0; 598.1749 (77.5), 598.1728 (84.6), 3.5.

Single isomer synthesis of $\mathbf{4 d}$. To separate oven-dried test tubes, $3(0.099 \mathrm{~g}, 0.135 \mathrm{mmol})$ in $\mathrm{CHCl}_{3}(2.02 \mathrm{~g})$ and DBU $(0.192 \mathrm{~g}, 1.261 \mathrm{mmol})$ in $\mathrm{CHCl}_{3}(2.01 \mathrm{~g})$ were added to a $0{ }^{\circ} \mathrm{C}$ cold bath 
and allowed to equilibrate. After 10 minutes, the heterogeneous allyl solution was quickly added to the DBU solution to become homogeneous and pale yellow after a few seconds. The reaction was removed from the cold bath and glovebox after 10 minutes and allowed to warm to room temperature for 10 minutes. The solution was diluted with $75 \mathrm{~mL} \mathrm{Et}_{2} \mathrm{O}$, extracted with $5 \times 25 \mathrm{~mL}$ of $\mathrm{NaOH}\left(1 \mathrm{M}\right.$, aqueous), back-extracted with $2 \times 25 \mathrm{~mL} \mathrm{Et}_{2} \mathrm{O}$, dried with $\mathrm{MgSO}_{4}$, filtered through a $60 \mathrm{~mL}$ coarse porosity fritted funnel, and the solvent removed to produce a yellow solid. The material was moved to the a tared 4 dram vial with DCM (5x1 mL), and the solvent removed to produce a yellow solid that was free flowing once scraped with a spatula $(0.073 \mathrm{~g}$, $0.125 \mathrm{mmol}, 93 \%$ yield). The solid was pure via NMR and produced a single isomer of the cyclohexadiene complex (10:1 cdr).

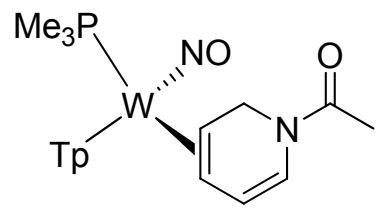

TpW(NO)(PMe $\left.)_{3}\right)\left(3,4-\eta^{2}\right.$-(1-(pyridin-1(2H)-yl)ethanone)). 7d. A solution of HOTf $(0.121 \mathrm{~g}, 0.806$ $\mathrm{mmol})$ in $\mathrm{MeCN}(6.31 \mathrm{~g})$ was added to a vial containing $7 \mathrm{p}(0.501 \mathrm{~g}, 0.800 \mathrm{mmol})$ to make a yellow homogenous solution. After 1 minute, the solution was transferred to a vial containing morpholine ( $0.087 \mathrm{~g}, 0.999 \mathrm{mmol})$. After 10 minutes, a some crystalline material began coating the reaction vial. The solution remained undisturbed for $23 \mathrm{~h}$, when the reaction solution was decanted away from the solid. The precipitate was washed with MeCN ( $2 \times \sim 0.3 \mathrm{~g})$ and placed under vacuum. After several days under vacuum, the yellow crystalline material was scraped from the reaction vial and transferred to a new vial $\left(0.364 \mathrm{~g}, 0.581 \mathrm{mmol}, 73 \%\right.$ yield). ${ }^{1} \mathrm{H}$ NMR $\left(\mathrm{CDCl}_{3}, \delta\right): 8.24(\mathrm{~d}, J=2.0,1 \mathrm{H}, \mathrm{PzA} 3), 8.02(\mathrm{~d}, J=2.0,1 \mathrm{H}, \mathrm{PzB} 3), 7.71(\mathrm{~m}, 2 \mathrm{H}, \mathrm{PzB} 5 / \mathrm{Tp}), 7.58(\mathrm{~d}, J$ $=2.0,1 \mathrm{H}, \mathrm{Tp}), 7.31(\mathrm{~d}, J=2.0,1 \mathrm{H}, \mathrm{PzC}), 6.29(\mathrm{t}, J=2.0,1 \mathrm{H}, \mathrm{PzB} 4), 6.19(\mathrm{t}, J=2.0,2 \mathrm{H}$, PzA4/PzB4), 6.14 (dd, $J=7.4,5.8,1 \mathrm{H}, \mathrm{H} 5), 5.95$ (d, $J=7.4,1 \mathrm{H}, \mathrm{H} 6), 5.34$ (d, $J=13.0,1 \mathrm{H}$, H2(syn)), 4.63 (dd, $J=13.0,3.2,1 \mathrm{H}, \mathrm{Hs}$ (anti)), 2.97 (ddd, $J=10.8,10.2,3.2,1 \mathrm{H}, \mathrm{H} 3$ ), 2.15 (s, 3H, Amide-Me), 1.63 (ddd, $J=10.2,5.8,1.8,1 \mathrm{H}, \mathrm{H} 4), 1.24$ (d, $\left.J=8.2,9 \mathrm{H}, \mathrm{PMe}_{3}\right) .{ }^{13} \mathrm{C} \mathrm{NMR}\left(\mathrm{CDCl}_{3}, \delta\right)$ : 169.1 (Amide-CO), 144.3 (PzA3), 143.6 (PzB3), 140.2 (PzC3), 136.6 (Tp), 136 (Tp), 135.2 (Tp), 119.8 (C5), 117.8 (C6), 106.4 (PzB4), 105.9/105.7 (PzA4/PzC4), 59.4 (C3, d, J = 13.4), 45.7 (C4), 44.2 (C2), 23.2 (Amide-Me), $13.7\left(\mathrm{PMe}_{3}, \mathrm{~d}, J=27.9\right) .{ }^{31} \mathrm{P} \mathrm{NMR}\left(\mathrm{CDCl}_{3}, \delta\right):-9.19\left(J_{\mathrm{WP}}=281\right)$. IR: $v_{\mathrm{BH}}$ $=2488 \mathrm{~cm}^{-1}, v=1643 \mathrm{~cm}^{-1}, v=1616 \mathrm{~cm}^{-1}, v_{\mathrm{NO}}=1562 \mathrm{~cm}^{-1}$. CV (DMA): $E_{\mathrm{p}, \mathrm{a}}=+0.34 \mathrm{~V}$. ESI-MS: obs'd (\%), calc'd (\%), ppm, $(\mathrm{M}+\mathrm{Na})^{+}: 647.1559$ (80.8), 647.155 (85.9), 1.5; 648.1585 (91.6), 648.1575 (79.6), 1.5; 649.158 (100), 649.1573 (100), 1.1; 650.1621 (39.9), 650.1616 (41.2), 0.7; 651.1618 (94.6), 651.1606 (84.6), 1.9. Anal. Calc'd for $\mathrm{C}_{19} \mathrm{H}_{28} \mathrm{BN}_{8} \mathrm{O}_{2} \mathrm{PW}: \mathrm{C}, 36.45 ; \mathrm{H}, 4.51 ; \mathrm{N}$, 17.90; Found: $\mathrm{C}, 36.60 ; \mathrm{H}, 4.52 ; \mathrm{N}, 17.94$. 


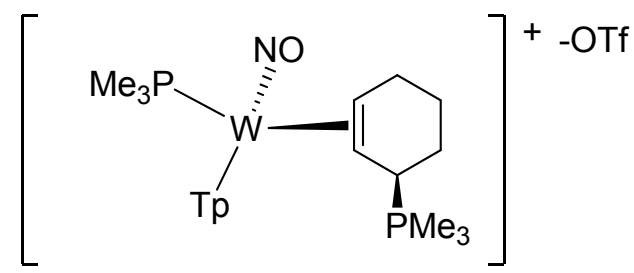

[TpW(NO)(PMe $)\left(2,3-\eta^{2}\right.$-( cyclohex-2-en-1-yltrimethylphosphonium)](OTf). 8c. $\mathrm{PMe}_{3}(0.037 \mathrm{~g}$, $0.473 \mathrm{mmol}$ ) was added to a heterogeneous yellow solution of $3(0.036 \mathrm{~g}, 0.049 \mathrm{mmol})$ in $\mathrm{CHCl}_{3}$ $(2.01 \mathrm{~g})$ to become homogeneous and pale yellow. After two minutes, the stirring reaction solution was diluted with $20 \mathrm{~mL} \mathrm{Et}{ }_{2} \mathrm{O}$ to precipitate a white solid that was collected on a $15 \mathrm{~mL}$ medium porosity fritted funnel. The white residue on the reaction flask was dissolved in $1 \mathrm{~mL}$ $\mathrm{CHCl}_{3}$ and precipitated with $20 \mathrm{mLEt}{ }_{2} \mathrm{O}$. The solid was collected on the same $15 \mathrm{~mL}$ medium porosity fritted funnel, washed with $2 \times 7 \mathrm{~mL} \mathrm{Et}_{2} \mathrm{O}$ and placed under vacuum $(0.022 \mathrm{~g}, 0.027$ mmol, 55 \% yield). ${ }^{1} \mathrm{H}$ NMR $\left(\mathrm{CDCl}_{3}, \delta\right): 8.00$ (d, $\left.J=2.0,1 \mathrm{H}, \mathrm{PzB} 3\right), 7.98(\mathrm{~d}, J=2.0,1 \mathrm{H}, \mathrm{PzA} 3), 7.74$ (d, J = 2.0,1H, PzC5), 7.70 (d, J=2.0,1H, PzB5), 7.68 (d, $J=2.0,1 \mathrm{H}, \mathrm{PzA} 5), 7.29$ (d, $J=2.0,1 \mathrm{H}$, PzC3), 6.30+6.29 (t, $J=2.0,2 \mathrm{H}, \mathrm{PzB} 4 / \mathrm{PzC} 4), 6.22(\mathrm{t}, J=2.0,1 \mathrm{H}, \mathrm{PzA} 4), 3.60\left(\mathrm{ddd},{ }^{2} J_{\mathrm{PH}}=8.9, J=\right.$ 6.7, 6.7, 1H, H1), $3.24(\mathrm{~m}, 1 \mathrm{H}, \mathrm{H} 4), 2.77\left(\mathrm{~m}, 2 \mathrm{H}, \mathrm{H} 3+\mathrm{H} 4{ }^{\prime}\right), 2.07(\mathrm{~m}, 1 \mathrm{H}, \mathrm{H} 6), 1.87(\mathrm{~m}, 1 \mathrm{H}, \mathrm{H} 5), 1.64$ $\left(\mathrm{d},{ }^{2} \mathrm{~J}_{\mathrm{PH}}=13.3,9 \mathrm{H}, \mathrm{C}-\mathrm{PMe}_{3}\right), 1.60\left(\mathrm{~m}, 1 \mathrm{H}, \mathrm{H} 6^{\prime}\right), 1.5\left(\mathrm{~m}, 1 \mathrm{H}, \mathrm{H} 5^{\prime}\right), 1.22\left(\mathrm{~d},{ }^{2} J_{\mathrm{PH}}=8.3,9 \mathrm{H}, \mathrm{W}-\mathrm{PMe}_{3}\right)$, $0.62\left(\mathrm{dd},{ }^{3} J_{\mathrm{PH}}=22.9, J=11.1,1 \mathrm{H}, \mathrm{H} 2\right) .{ }^{13} \mathrm{C} \mathrm{NMR}\left(\mathrm{CDCl}_{3}, \delta\right): 143.3$ (PzB3), 141.9 (PzA3), 140.1 (PzC3), 137.5 (PzA5), 137.1 (PzC5), 136.6 (PzB5), 129.9 (q, ${ }^{1} \mathrm{~J}_{\mathrm{FC}}=320$, triflate), 107.0/106.7/106.6 $(P z A 4 / P z B 4 / P z C 4), 49.7\left(d,{ }^{2} J_{P C}=11.3, C 3\right), 45.4\left(d, J_{P C}=3.0, C 2\right), 33.4\left(d,{ }^{1} J_{P C}=42.6, C 1\right), 29.1$ $\left(d, J_{P C}=3.3, C 4\right), 23.1\left(d,{ }^{2} J_{P C}=3.6, C 6\right), 21.4\left(d,{ }^{3} J_{P C}=9.5, C 5\right), 13.8\left(d,{ }^{1} J_{P C}=28.3, W-P M e_{3}\right), 6.9$ $\left.\left(d,{ }^{1} J_{\mathrm{PC}}=53.5, \mathrm{C}-\mathrm{PMe}\right)_{3}\right) .{ }^{31} \mathrm{P} \mathrm{NMR}\left(\mathrm{CDCl}_{3}, \delta\right):-10.04\left(J_{\mathrm{WP}}=286\right), 35.41\left(\mathrm{C}-\mathrm{PMe}_{3}\right) . \mathrm{IR}: \mathrm{V}_{\mathrm{BH}}=2481$ $\mathrm{cm}^{-1}, \mathrm{v}_{\mathrm{NO}}=1542 \mathrm{~cm}^{-1}$. CV (MeCN): $E_{\mathrm{p}, \mathrm{a}}=+0.54 \mathrm{~V}$. ESI-MS: obs'd (\%), calc'd (\%), ppm, $\mathrm{M}+$ : 658.2128 (81.8), 658.2114 (85.1), 2.1; 659.2139 (95.5), 659.214 (80.1), 0.1; 660.2156 (100), 660.2138 (100), 2.7; 661.2296 (31.8), 661.2180 (42.3), 17.5; 662.2158 (72.7), 662.2171 (84), 1.9.

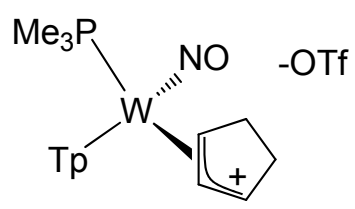

[TpW(NO)(PMe $\left.{ }_{3}\right)\left(2,3-\eta^{2}\right.$-cyclopent-2-en-ylium)][OTf]. 9. A solution of HOTf $(0.058 \mathrm{~g}, 0.386$ $\mathrm{mmol})$ in $\mathrm{MeCN}(0.25 \mathrm{~g})$, was added to a solution of $10 \mathrm{p}, \mathrm{d}(0.195 \mathrm{~g}, 0.343 \mathrm{mmol})$ in $\mathrm{MeCN}(0.33$ g) to make a deep yellow homogeneous solution. After $30 \mathrm{~s}$, the reaction solution was added to $125 \mathrm{~mL}$ of stirring $\mathrm{Et}_{2} \mathrm{O}$ to precipitate a tan-yellow solid that was collected on a $15 \mathrm{~mL}$ medium porosity fritted funnel, washed with $2 \times 7 \mathrm{~mL} \mathrm{Et}{ }_{2} \mathrm{O}$, and placed under vacuum $(0.183 \mathrm{~g}, 0.254$ mmol, $74 \%$ yield). CV (MeCN): $E_{p, a}=+1.77 \mathrm{~V}, E_{\mathrm{p}, \mathrm{c}}=-0.93 \mathrm{~V}$. Characterization for 9 has previously been published. 


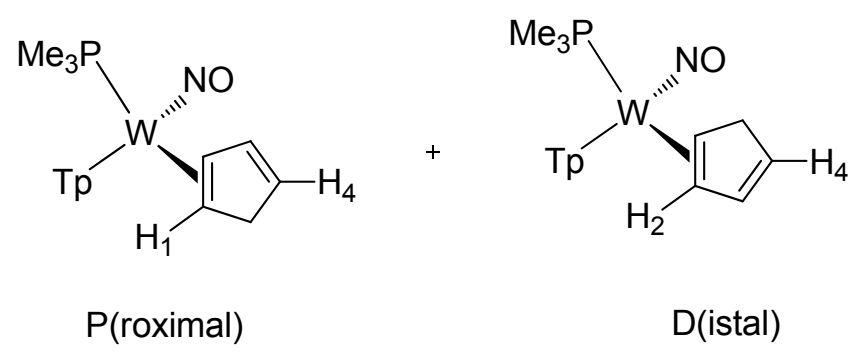

TpW(NO)(PMe $)\left(1,2-\eta^{2}\right.$-cyclopenta-1,3-diene): 10p, 10d. To a flame-dried test tube with a stir bar was added $\mathrm{TpW}(\mathrm{NO})\left(\mathrm{PMe}_{3}\right)\left(\eta^{2}\right.$-benzene) $(0.100 \mathrm{~g}, 0.172 \mathrm{mmol})$ and $\mathrm{DME}(2.0 \mathrm{~g})$, to give a homogeneous yellow solution, to which cyclopentadiene $(0.212 \mathrm{~g}, 3.207 \mathrm{mmol})$ was added. The test tube was placed in a $67^{\circ} \mathrm{C}$ oil bath for 16 hours. A dark brown solution was cooled to room temperature and precipitated over a mixture of $37 \mathrm{~mL}$ hexanes and $13 \mathrm{~mL}$ ether. A brown/purple precipitate was filtered using a $15 \mathrm{~mL}$ fine-porosity fritted funnel and discarded. The light yellow filtrate was evaporated to dryness and dissolved in minimal dichloromethane for transfer to a 4 dram vial with stirbar. The dichloromethane was evaporated and $2 \mathrm{~mL}$ ether added to the vial and allowed to stir overnight. An orange liquid was carefully pipeted out of the vial, leaving a pale tan solid. One $\mathrm{mL}$ of hexanes and 5 drops ether was added to the vial and allowed to stir overnight. One $\mathrm{mL}$ of hexanes was added to the vial and the pale tan solid was filtered over a $2 \mathrm{~mL}$ fine-porosity fritted funnel to give $0.058 \mathrm{~g}(0.102 \mathrm{mmol}, 60 \%$ yield $) .{ }^{1} \mathrm{H}$ $\operatorname{NMR}\left(\mathrm{CDCl}_{3}, \delta\right): 8.42$ (d, J = 1.7, 1H, PzA3(p)), 8.25 (d, J = 1.7, 1H, PzA3(d)), 8.05 (d, J = 1.8, $1 \mathrm{H}$, PzB3(p)), $8.04(d, 1 H, P z B 3(d)), 7.72(m, 1 H, P z B 5(p+d)), 7.67(m, 1 \mathrm{H}, \operatorname{PzC}(p+d)), 7.61(d, J=2.0$, 1H, PzA5(p)), $7.59(d, 1 H, \operatorname{PzA5}(d)), 7.28(m, 1 \mathrm{H}, \operatorname{PzC5}(p+d)), 6.29(\mathrm{~m}, 1 \mathrm{H}, \operatorname{PzB} 4(\mathrm{p}+\mathrm{d})), 6.23(\mathrm{~m}$, $1 \mathrm{H}, \operatorname{PzA} 4(\mathrm{p}+\mathrm{d})), 6.15(\mathrm{~m}, 1 \mathrm{H}, \mathrm{PzC} 4(\mathrm{p}+\mathrm{d})), 1.31\left(\mathrm{~d}, J=7.5,9 \mathrm{H}, \mathrm{PMe}_{3}(\mathrm{p})\right), 1.27(\mathrm{~d}, J=7.7,9 \mathrm{H}$, $\left.\mathrm{PMe}_{3}(\mathrm{~d})\right)$, Proximal diene ( $\mathrm{p}$ - Major isomer): $6.41(\mathrm{dd}, J=4.8,1.9,1 \mathrm{H}, \mathrm{H} 3), 5.37(\mathrm{~m}, 1 \mathrm{H}, \mathrm{H} 4)$, $4.50(\mathrm{~m}, 1 \mathrm{H}, \mathrm{H} 5), 3.95\left(\mathrm{~m}, 1 \mathrm{H}, \mathrm{H} 5^{\prime}\right), 3.72(\mathrm{dd}, J=12.5,7.3,1 \mathrm{H}, \mathrm{H} 2), 2.15(\mathrm{~m}, 1 \mathrm{H}, \mathrm{H} 1)$, Distal diene (d - minor isomer): $6.68(\mathrm{dd}, J=5.0,2.1,1 \mathrm{H}, \mathrm{H} 3), 5.30(\mathrm{~m}, 1 \mathrm{H}, \mathrm{H} 4), 4.54(\mathrm{~m}, 1 \mathrm{H}, \mathrm{H5}), 3.54(\mathrm{~m}$, $1 \mathrm{H}, \mathrm{H5}^{\prime}$ ), 3.40 (dt, $\left.J=14.0,7.0,1 \mathrm{H}, \mathrm{H} 1\right), 2.44$ (dd, $\left.J=7.4,2.3,1 \mathrm{H}, \mathrm{H} 2\right) .{ }^{13} \mathrm{C} \mathrm{NMR}\left(\mathrm{CDCl}_{3}, \delta\right): 144.4$ (Tp), 144.3 (Tp), 143.9 (Tp), 141.2 (Tp), 140.9 (Tp), 140.6 (Tp), 136.3 (Tp), 136.2 (Tp), 135.8 (Tp), 135.7 (Tp), 135.1 (Tp), 134.8 (Tp), 106.4 (Tp), 106.3 (Tp), 105.9 (Tp), 105.7 (Tp), 105.6 (Tp), $105.5(\mathrm{Tp}), 14.5\left(\mathrm{~d}, \mathrm{~J}_{\mathrm{P}-\mathrm{C}}=27.5,3 \mathrm{C}, \mathrm{PMe}_{3}\right), 14.1\left(\mathrm{~d}, \mathrm{~J}_{\mathrm{PC}}=27.1,3 \mathrm{C}, \mathrm{PMe}_{3}\right)$, Proximal diene: 136.4 (C3), 123.0 (C4), 67.9 (C2), 57.3 (C1), 43.0 (C5), Distal diene: 138.0 (C3), 121.9 (C4), 66.4 (C2), $58.6(C 1), 43.2(C 5) \cdot{ }^{31} \mathrm{P} \mathrm{NMR}\left(\mathrm{CDCl}_{3}, \delta\right):-11.11\left(J_{\mathrm{WP}}=280\right),-11.88\left(J_{\mathrm{WP}}=290\right)$. IR: $v_{\mathrm{BH}}=2484 \mathrm{~cm}^{-}$ ${ }^{1}, v_{\mathrm{NO}}=1554 \mathrm{~cm}^{-1} . \mathrm{CV}(\mathrm{DMA}): E_{\mathrm{p}, \mathrm{a}}=+0.36$ V. HRMS: Overlapping signals for hydride loss $(\mathrm{M}-\mathrm{H})+$ and protonation $(\mathrm{M}+\mathrm{H})+$ complicated the spectrum and caused overlapping $\mathrm{M} / \mathrm{Z}$ peaks to not fit within the acceptable 5 ppm difference from that of the calculated. ESI-MS ((M-H)+): obs'd (\%), calc'd (\%), ppm: 566.1355 (61.8), 566.1359 (87.3), 0.7; 567.139 (56.2), 567.1385 (79.1), 0.9; 568.1419 (100), 568.1382 (100), 6.5; 569.1484 (45.2), 569.1427 (39.3), 10.0; 570.1451 (89.3), 570.1415 (85.2), 6.3. ESI-MS ((M+H) $)$ : 568.1419 (112), 568.1515 (87.3), 16.9; 569.1484 (50.7), 569.1541 (79.1), 10.1; 570.1451 (100), 570.1538 (100), 15.4; 571.1514 (22.7), 571.1583 (39.3), 12.2; 572.1573 (36.2), 572.1571 (85.2), 0.3. 

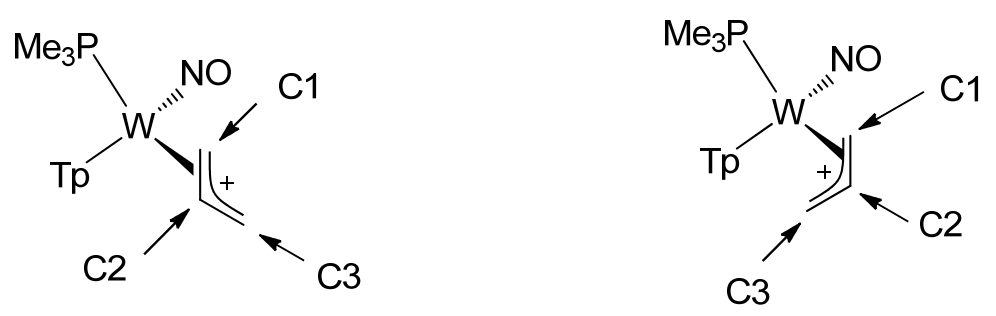

[TpW(NO)(PMe $)\left(2,3-\eta^{2}\right.$-propan-1-ylium)][OTf]. exo-11, endo-11. Diallyl ether (0.92 g, 9.4 $\mathrm{mmol}$ ) was added to a flame dried test tube containing $\mathrm{TpW}(\mathrm{NO})\left(\mathrm{PMe}_{3}\right)\left(\eta^{2}\right.$-benzene) $(0.506 \mathrm{~g}$, $0.871 \mathrm{mmol})$ in DME $(2.62 \mathrm{~g})$ to make a homogeneous yellow solution that was allowed to stir in a $57{ }^{\circ} \mathrm{C}$ oil bath. After $1.5 \mathrm{~h}$, the dark purple-brown solution was removed from the warm bath. A solution of HOTf $(0.127 \mathrm{~g}, 0.846 \mathrm{mmol})$ in $\mathrm{MeCN}(0.640 \mathrm{~g})$ was added to the reaction solution to make a dark yellow solution. The reaction solution was placed in a $0{ }^{\circ} \mathrm{C}$ cold bath overnight. After $15 \mathrm{~h}$, the solution was removed from the cold bath and added to $100 \mathrm{~mL}$ of stirring $\mathrm{Et}_{2} \mathrm{O}$. The tan-yellow precipitate was then collected on a $30 \mathrm{~mL}$ medium porosity fritted funnel, washed with $2 \times 15 \mathrm{~mL} \mathrm{Et}{ }_{2} \mathrm{O}$ and placed under vacuum $(0.298 \mathrm{~g}, 0.430 \mathrm{mmol}, 49 \%$ yield). ${ }^{1} \mathrm{H}$ NMR $\left(\mathrm{CD}_{3} \mathrm{CN}, \delta\right): 8.4(\mathrm{~d}, J=2.0,1 \mathrm{H}, \mathrm{PzB} 3(\mathrm{exo})), 8.16(\mathrm{~d}, J=2.0,1 \mathrm{H}, \mathrm{PzA} 3(\mathrm{exo})), 8.1(\mathrm{~s}(\mathrm{br}), 2 \mathrm{H}$, 2 Tp's), 8.08 (d, $J=2.0,1 \mathrm{H}, \mathrm{PzB} 3($ endo)), 8.00/7.99 (m, 2H, PzC5(exo)/Tp), 7.98 (d, $J=2.0,1 \mathrm{H}$, PzA3(endo)), 7.89 (m, 2H, PzC3(exo)/Tp), $7.81(\mathrm{~d}, J=2.0,1 \mathrm{H}, \operatorname{PzA} 5(\mathrm{exo})), 7.71(\mathrm{~d}, J=2.0,1 \mathrm{H}$, $\mathrm{PzC}($ endo)), 6.55/6.54/6.52/6.50 ( $\mathrm{t}, J=2.0,4 \mathrm{H}, \mathrm{PzC} 4($ endo) $/ 3 \mathrm{Tp} 4 \mathrm{~s}), 6.38(\mathrm{t}, J=2.0,1 \mathrm{H}$, PzC4(exo)), 6.35 (t, $J=2.0,1 \mathrm{H}, \operatorname{PzA} 4($ exo $)), 1.24\left(\mathrm{~d}, J=10.4,9 \mathrm{H}, \mathrm{PMe}_{3}(\mathrm{exo})\right), 1.21$ (d, $J=10.1$, $9 \mathrm{H}, \mathrm{PMe}_{3}$ (endo)), Exo Isomer: 5.36 (ddddd, $J=14.6,13.3,8.3,8.0,{ }^{3} J_{\mathrm{PH}}=1.8,1 \mathrm{H}, \mathrm{H} 3$ ), 5.05 (ddd, $J=14.6,1.0,{ }^{3} J_{\mathrm{PH}}=2.6,1 \mathrm{H}, \mathrm{H} 5$ ), 4.83 (dddd, $\left.J=8.3,2.9,1.1,{ }^{3} J_{\mathrm{PH}}=1.2,1 \mathrm{H}, \mathrm{H} 4\right), 3.83$ (dddd, $J=$ 8.0, 2.9, 2.9, ${ }^{3} J_{\mathrm{PH}}=13.4,1 \mathrm{H}, \mathrm{H} 2$ ), 2.57 (ddddd, $J=13.3,2.9,1.1,1.0,{ }^{3} J_{\mathrm{PH}}=8.8,1 \mathrm{H}, \mathrm{H} 1$ ), Endo Isomer: 6.33 (ddddd, $J=14.3,10.5,7.8,7.4,{ }^{3} J_{\mathrm{PH}}=1.1,1 \mathrm{H}, \mathrm{H} 3$ ), 4.53 (ddddd, $J=7.8,3.2,1.2$, $0.8,3 \mathrm{JPH}=1.0,1 \mathrm{H}, \mathrm{H} 4$ ), 3.70 (dddd, $J=7.4,3.6,3.2,3 \mathrm{JPH}=14.3,1 \mathrm{H}, \mathrm{H} 2$ ), 3.60 (dddd, $J=14.3$, $1.2,0.5,{ }^{3} J_{\mathrm{PH}}=1.9,1 \mathrm{H}, \mathrm{H} 5$ ), 2.35 (ddddd, $J=10.5,3.6,0.8,0.5,3 \mathrm{JPH}=9.4,1 \mathrm{H}, \mathrm{H} 1$ ). ${ }^{13} \mathrm{C} \mathrm{NMR}$ $\left(\mathrm{CD}_{3} \mathrm{CN}, \delta\right.$ ): 149.0 (PzA3(exo)), 147.9 (PzA3(endo)), 146.9 (d, $\left.{ }^{4} J_{\mathrm{PC}}=2.4, \mathrm{PzB} 3(\mathrm{exo})\right), 145.3\left(\mathrm{~d},{ }^{4} \mathrm{~J}_{\mathrm{PC}}\right.$ = 2.4, PzB3(endo)), 144.4 (PzC3(endo)), 144.1 (PzC3(exo)), 140.5/140.1/139.8/139.2 (6 Tp5's), 109.4/108.8/108.6/108.4/108.1 (6 Tp4's), 13.7 (d, $J_{\mathrm{PC}}=33.8, \mathrm{PMe}_{3}(\mathrm{exo})$ ), 13.0 (d, $\mathrm{J}_{\mathrm{PC}}=33.8$, $\left.\mathrm{PMe}_{3}(\mathrm{endo})\right)$, Allyl ligand signals for the Exo Isomer: 115.1 (d, $\left.J_{\mathrm{PC}}=5.8, \mathrm{C} 3\right), 100.9\left(\mathrm{~d}, J_{\mathrm{PC}}=2.4\right.$, $\mathrm{C2}$ ), $60.7\left(\mathrm{~d}, J_{\mathrm{PC}}=11.3, \mathrm{C} 1\right)$. Allyl ligand signals for the Endo Isomer: $120.6\left(\mathrm{~d}, J_{\mathrm{PC}}=5.8, \mathrm{C} 3\right), 100.1$ $\left(d, J_{\mathrm{PC}}=1.8, \mathrm{C} 2\right), 62.3\left(\mathrm{~d}, J_{\mathrm{PC}}=12.5, \mathrm{C} 1\right) .{ }^{31} \mathrm{P} N M R\left(\mathrm{CD}_{3} \mathrm{CN}, \delta\right):-2.91\left(J_{\mathrm{WP}}=252\right),-7.40\left(J_{\mathrm{WP}}=256\right)$. IR: $v_{\mathrm{BH}}=2515 \mathrm{~cm}^{-1}, v_{\mathrm{NO}}=1647 \mathrm{~cm}^{-1} . \mathrm{CV}(\mathrm{MeCN}): E_{\mathrm{p}, \mathrm{a}}=+2.07 \mathrm{~V}, E_{\mathrm{p}, \mathrm{c}}=-1.08 \mathrm{~V}$. ESI-MS: obs'd (\%), calc'd (\%), ppm, (M-OTf) ${ }^{+}: 542.1376$ (84.4), 542.1359 (88.4), 3.2; 543.1397 (64.4), 543.1384 (78.5), 2.3; 544.1392 (100), 544.1381 (100), 1.9; 545.1441 (32.2), 545.1427 (37.8), 2.5; 546.1428 (87.2), 546.1414 (85.9), 2.6. 


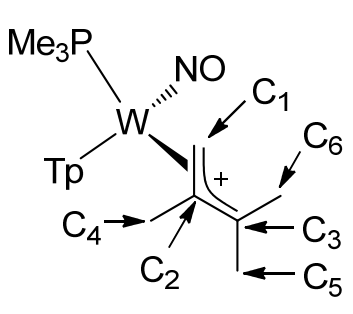

Exo

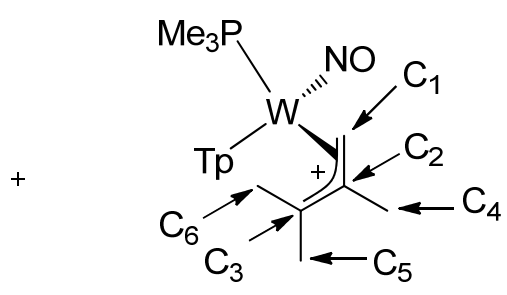

Endo

TpW(NO)(PMe $)\left(1,2-\eta^{2}\right.$-(2,3-dimethylbut-3-en-2-ylium)][OTf]. exo-12, endo-12. In a flame dried test tube, 2,3-dimethylbutadiene $(0.91 \mathrm{~g}, 11.1 \mathrm{mmol})$ was added to a homogeneous yellow solution of TpW(NO) $\left(\mathrm{PMe}_{3}\right)\left(\eta^{2}\right.$-benzene) $(0.501 \mathrm{~g}, 0.862 \mathrm{mmol})$ in DME $(2.62 \mathrm{~g})$. The tube was added to a $57{ }^{\circ} \mathrm{C}$ oil bath and allowed to stir. The dark purple-brown solution was removed from the warm bath after $1.5 \mathrm{~h}$. An HOTf $(0.130 \mathrm{~g}, 0.866 \mathrm{mmol})$ in MeCN $(0.62 \mathrm{~g})$ was added to the solution to make a dark yellow solution. After 45 minutes the solution becaome heterogeneous. The solution was allowed to stir for an additional $15 \mathrm{~h}$ and the yellow precipitate was collected on a $15 \mathrm{~mL}$ medium porosity fritted funnel. The precipitate was washed with $~ 3 \times 0.3 \mathrm{~g}$ DME and placed under vacuum $(0.298 \mathrm{~g}, 0.405 \mathrm{mmol}, 47 \%$ yield $) .{ }^{1} \mathrm{H}$ $\operatorname{NMR}\left(\mathrm{CD}_{3} \mathrm{CN}, \delta\right)$ : Endo: 8.28 (d, $\left.J=2.0,1 \mathrm{H}, \mathrm{PzC} 3\right), 8.18(\mathrm{~d}, J=2.0,1 \mathrm{H}, \mathrm{PzB} 3), 8.04(\mathrm{~d}, J=2.0,1 \mathrm{H}$, PzC5), $7.92(\mathrm{~d}, J=2.0,1 \mathrm{H}, \mathrm{PzA} 5), 7.87(\mathrm{~d}, J=2.0,1 \mathrm{H}, \mathrm{PzB} 5), 7.67(\mathrm{~d}, J=2.0,1 \mathrm{H}, \mathrm{PzA} 3), 6.55(\mathrm{t}, J=$ 2.0, $1 \mathrm{H}, \mathrm{PzC} 4), 6.40$ (m(overlap), $2 \mathrm{H}, \mathrm{PzB} 4), 6.35$ (t, $J=2.0,1 \mathrm{H}, \mathrm{PzA} 4), 3.36$ (dd, $J=7.2,{ }^{3} J_{\mathrm{PH}}=$ 10.9, $1 \mathrm{H}, \mathrm{H} 1$ ), $3.12\left(\mathrm{dd}, J=7.2,{ }^{3} \mathrm{~J}_{\mathrm{PH}}=10.1,1 \mathrm{H}, \mathrm{H} 1^{\prime}\right), 2.25(\mathrm{~s}, 3 \mathrm{H}, \mathrm{H} 6), 1.92(\mathrm{~s}, 3 \mathrm{H}, \mathrm{H} 5) 1.34(\mathrm{~s}, 3 \mathrm{H}$, $\mathrm{H} 4), 1.27\left(\mathrm{~d}, J_{\mathrm{PH}}=9.8,9 \mathrm{H}, \mathrm{PMe}_{3}\right.$ ). Exo: $8.07(\mathrm{~m}, 2 \mathrm{H}, \mathrm{PzC} 5 / \mathrm{PzC} 3), 7.99(\mathrm{~d}, J=2.0,1 \mathrm{H}, \mathrm{PzA} 5), 7.93$ (d, $J=2.0,1 \mathrm{H}, \mathrm{PzB} 3$ ), 7.91 (d, $J=2.0,1 \mathrm{H}, \mathrm{PzB} 5), 7.83$ (d, $J=2.0,1 \mathrm{H}, \mathrm{PzA} 3), 6.53$ (t, $J=2.0,1 \mathrm{H}$, PzC4), 6.4 (m(overlap), 1H, PzA4), 6.38 (t, $J=2.0,1 \mathrm{H}, \mathrm{PzB} 4), 3.71$ (dd, $J=6.3,{ }^{3} J_{\mathrm{PH}}=15.2,1 \mathrm{H}$, $\mathrm{H} 1), 2.80\left(\mathrm{dd}, J=6.3,{ }^{3} J_{\mathrm{PH}}=5.7,1 \mathrm{H}, \mathrm{H} 1^{\prime}\right), 2.38(\mathrm{~s}, 3 \mathrm{H}, \mathrm{H} 6), 1.78(\mathrm{~s}, 3 \mathrm{H}, \mathrm{H} 5), 1.28\left(\mathrm{~d}, J_{\mathrm{PH}}=9.8,9 \mathrm{H}\right.$, $\left.\mathrm{PMe}_{3}\right), 1.00$ (s, 3H, H4). ${ }^{13} \mathrm{C} N M R\left(\mathrm{CD}_{3} \mathrm{CN}, \delta\right)$ : Endo: 176.8 (C3), 147.9 (PzA3), 145.8 (d, J = 2.4, PzB3), 145.1 (PzC3), 140.7 (PzA5), 139.6/139.5 (PzB5/PzC5), 122.2 (q, $J_{C F}=321 \mathrm{~Hz}$, Triflate), 108.7 (PzB4), 108.4 (PzC4), 108.2 (PzA4), 102.3 (d, $\left.J_{P C}=3.8, C 2\right), 65.2$ (d, $\left.J_{P C}=14.2, C 1\right), 29.1$ (C5), 27.0 (C6), 22.0 (C4), 13.0 (d, 1 J $J_{P C}=32.6$, PMe3). Exo: 152.8 (C3), 147.4 (PzA3), 145.4/145.3 (PzB3/PzC3), 141.5 (PzA5), 139.9/139.8 (PzB5/PzC5), $112.6\left(\mathrm{~d}, J_{\mathrm{PC}}=4.6, \mathrm{C} 2\right), 108.8(\mathrm{PzC} 4)$, 108.6/108.5 (PzA4/BzB4), 65.2 (d, JPC 14.2, C1), 27.4 (C5), 24.5 (C4), 24 (C6), $13.2\left(\mathrm{~d},{ }^{1} J_{P C}=\right.$ 32.8, $\left.\mathrm{PMe}_{3}\right) .{ }^{31} \mathrm{P} N M R\left(\mathrm{CD}_{3} \mathrm{CN}, \delta\right):-4.09\left(J_{\mathrm{WP}}=259\right),-7.35\left(J_{\mathrm{WP}}=258\right) . \mathrm{IR}: v_{\mathrm{BH}}=2511 \mathrm{~cm}^{-1}, v_{\mathrm{NO}}=$ $1624 \mathrm{~cm}^{-1}$. CV (MeCN): $E_{\mathrm{p}, \mathrm{a}}=+1.93 \mathrm{~V}, E_{1 / 2}=-0.78 \mathrm{~V}, E_{1 / 2}=-1.66 \mathrm{~V}$. ESI-MS: obs'd (\%), calc'd (\%), ppm, $\mathrm{M}^{+}: 584.1828$ (71.7), 584.1828 (86.8), 0.1; 585.1858 (65.7), 585.1854 (79.3), 0.6; 586.1863 (100), 586.1852 (100), 1.9; 587.1899 (33), 587.1896 (40.1), 0.5; 588.1903 (62.3), 588.1884 (84.9), 3.1. Anal. Calc'd for $\mathrm{C}_{19} \mathrm{H}_{30} \mathrm{BF}_{3} \mathrm{~N}_{7} \mathrm{O}_{4}$ PSW: $\mathrm{C}, 31.04 ; \mathrm{H}, 4.11 ; \mathrm{N}, 13.34$; Found: $\mathrm{C}, 31.17 ; \mathrm{H}$, 4.29; N, 13.50. 


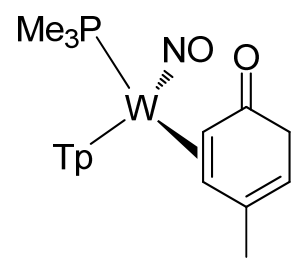

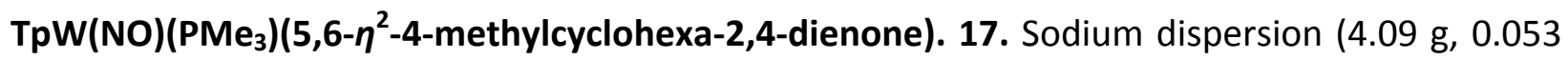
$\mathrm{mmol}, 30-35 \%$ in wax) was added to a $2 \mathrm{~L}$ round-bottom flask containing a stir bar and was stirred in $40 \mathrm{~mL}$ of hexanes for $20 \mathrm{~min}$. The hexanes was decanted. The sodium wax dispersion was stirred in an additional $40 \mathrm{~mL}$ of hexanes for $20 \mathrm{~min}$ and the hexanes decanted. Benzene $(400 \mathrm{~mL})$ was added to the round-bottom flask containing $\mathrm{TpW}(\mathrm{NO})(\mathrm{PMe}) \mathrm{Br}(5.997 \mathrm{~g}, 0.0103$ mol). After $24 \mathrm{~h}$, the reaction was filtered through $2 \mathrm{~cm}$ of Celite in a $350 \mathrm{~mL}$ medium porosity fritted funnel into a $2 \mathrm{~L}$ filter flask, containing a stirbar and $p$-cresol $(20.798 \mathrm{~g}, 0.1923 \mathrm{mmol})$. The Celite was washed with $200 \mathrm{~mL}$ of benzene. After $24 \mathrm{~h}$, the reaction mixture was chromatographed on silica $(3 \mathrm{~cm}$ ) in a $350 \mathrm{~mL}$ medium porosity fritted funnel by first eluting with toluene $(200 \mathrm{~mL})$, then $\mathrm{Et}_{2} \mathrm{O}(800 \mathrm{~mL})$, then EtOAc $(1 \mathrm{~L})$. A separate brown band came off of the column with each change in eluent. The EtOAc fraction solvent was removed in vacuo, dissolved in $30 \mathrm{~mL}$ of $\mathrm{DCM}$, and added to $500 \mathrm{~mL}$ of stirring hexanes. A tan precipitate was collected $\left(2.553 \mathrm{~g}, 0.0419 \mathrm{~mol}, 41 \%\right.$ yield). ${ }^{1} \mathrm{H}$ NMR $\left(\mathrm{CDCl}_{3}, \delta\right): 7.97$ (d, $\left.1 \mathrm{H}, J=2.0, \mathrm{PzB} 3\right), 7.92$ $(\mathrm{d}, 1 \mathrm{H}, J=2.0, \mathrm{PzA} 3), 7.80(\mathrm{~d}, 1 \mathrm{H}, J=2.0, \mathrm{PzC} 5), 7.72(\mathrm{~d}, 1 \mathrm{H}, J=2.0, \mathrm{PzB} 5), 7.64(\mathrm{~d}, 1 \mathrm{H}, J=2.0$, PzA5), $7.38(\mathrm{~d}, 1 \mathrm{H}, J=2.0, \mathrm{PzC} 3), 6.29$ (t overlaps with PzB4, $1 \mathrm{H}, J=2.0, \mathrm{PzC}$ ), 6.28 (t overlaps with PzC4, 1H, J = 2.0, PzB4), 6.17 (t, $1 \mathrm{H}, J=2.0, \mathrm{PzA} 4), 4.82$ (br s, 1H, H3), 3.51 (d, $1 \mathrm{H}, J=22.3$, $\mathrm{H} 2$ ), 3.37 (ddd, $\left.1 \mathrm{H},{ }^{3} J_{\mathrm{PH}}=12.0, J=9.1,2.5, \mathrm{H} 6\right), 2.97\left(\mathrm{~d}, 1 \mathrm{H}, J=22.3, \mathrm{H} 2^{\prime}\right), 1.93(\mathrm{~d}, 1 \mathrm{H}, J=9.1$, $\mathrm{H} 5), 1.55$ (s, 3H, Me), $1.26\left(\mathrm{~d}, 9 \mathrm{H},{ }^{2} \mathrm{~J}_{\mathrm{PH}}=8.9, \mathrm{PMe}_{3}\right) .{ }^{13} \mathrm{C} \mathrm{NMR}\left(\mathrm{CDCl}_{3}, \delta\right): 208.7$ (s, C1), 143.9 (s, PzA3), 143.6 (s, PzB3), 140.4 (s, PzC3), 139.3 (s, C4), 136.9 (s, PzC5) 136.3 (s, PzB5), 136.0 (s, PzA5), 111.6 (s, C3), 106.6 (s, PzB4 or PzC4), 106.4 (s, PzB4 or PzC4), 105.6 (s, PzA4), 64.9 (s, C5), $58.6\left(\mathrm{~d},{ }^{2} J_{\mathrm{PC}}=6.8, \mathrm{C} 6\right), 40.5(\mathrm{~s}, \mathrm{C} 2), 25.1(\mathrm{~s}, \mathrm{Me}), 13.1\left(\mathrm{~d},{ }^{1} \mathrm{~J}_{\mathrm{PC}}=28.3, \mathrm{PMe}_{3}\right) .{ }^{31} \mathrm{P} \mathrm{NMR}\left(\mathrm{CD}_{3} \mathrm{CN}, \delta\right)$ : $-12.30\left(J_{\mathrm{WP}}=273\right) . \mathrm{CV}(\mathrm{DMA}): E_{\mathrm{p}, \mathrm{a}}=+0.68 \mathrm{~V} . \mathrm{IR}: \mathrm{v}_{\mathrm{BH}}=2495 \mathrm{~cm}^{-1}, \mathrm{v}_{\mathrm{CO}}=1620 \mathrm{~cm}^{-1}, \mathrm{v}_{\mathrm{NO}}=1566 \mathrm{~cm}^{-}$ 1. ESI-MS: obs'd (\%), calc'd (\%), ppm, $(\mathrm{M}+\mathrm{H})^{+}: 610.1614$ (66.6), 610.1621 (86.1), 1.1; 611.1649 (73.4), 611.1647 (79.5), 0.3; 612.1639 (100), 612.1645 (100), 1.0; 613.1681 (39.3), 613.1688 (40.9), 1.1; 614.1685 (80.2), 614.1677 (84.7), 1.3.

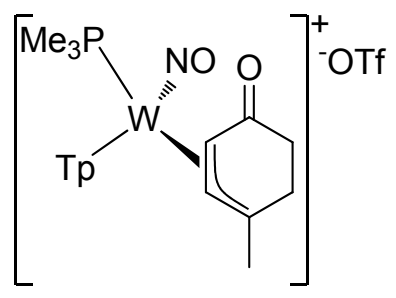

[TpW(NO)(PMe $)\left(2,3-\eta^{2}\right.$-1-methyl-4-oxocyclohex-2-en-1-ylium)][OTf]. 18. HOTf (0.018 g, 0.122 $\mathrm{mmol}$ ) in $1 / 2 \mathrm{~mL}$ MeCN was added to a vial containing 17 (0.050 g, $0.082 \mathrm{mmol})$ in $1 / 2 \mathrm{~mL}$ of $\mathrm{MeCN}$. After 5 minutes, the dark yellow homogeneous solution was added to $75 \mathrm{~mL}$ of stirring $\mathrm{Et}_{2} \mathrm{O}$ resulting in a brown precipitate. The precipitate was collected on a $15 \mathrm{~mL}$ fine porosity fritted funnel and rinsed with $3 \times 5 \mathrm{~mL} \mathrm{Et}{ }_{2} \mathrm{O}\left(0.040 \mathrm{~g}, 0.052 \mathrm{mmol}, 63 \%\right.$ yield). ${ }^{1} \mathrm{H} \mathrm{NMR}\left(\mathrm{CD}_{3} \mathrm{CN}\right.$, 
б): $8.16(\mathrm{~d}, 1 \mathrm{H}, J=2.0, \mathrm{PzB} 3), 8.10(\mathrm{~d}, 1 \mathrm{H}, J=2.0, \mathrm{PzC} 5), 8.02(\mathrm{~d}, 1 \mathrm{H}, J=2.0, \mathrm{PzC} 3), 7.97(\mathrm{~d}, 1 \mathrm{H}, J$ $=2.0$, PzB5), 7.94 (d, $1 \mathrm{H}, J=2.0, \mathrm{PzA} 5), 7.67(\mathrm{~d}, 1 \mathrm{H}, J=2.0, \mathrm{PzA} 3), 6.61(\mathrm{t}, 1 \mathrm{H}, J=2.0, \mathrm{PzC}$ ), 6.48 (t, $1 \mathrm{H}, J=2.0$, PzB4) $6.36(\mathrm{t}, 1 \mathrm{H}, J=2.0, \mathrm{PzA} 4), 4.74\left(\mathrm{ddd}, 1 \mathrm{H},{ }^{3} J_{\mathrm{PH}}=13.7, J=6.5,1.3, \mathrm{H3}\right), 4.69$ (dd, $1 \mathrm{H}, J=6.5,1.5, \mathrm{H} 2$ ), 3.33 (ddddd, $1 \mathrm{H}, J=21.7,9.8,2.1,1.5,1.3, \mathrm{H} 6$ ), 3.26 (ddd, $J=21.7,9.0$, 6.8, $1 \mathrm{H}, \mathrm{H6} 6^{\prime}$ ), 2.42 (ddd, $1 \mathrm{H}, J=19.0,9.8,6.8, H 5^{\prime}$ ), 2.25 (ddd, $\left.1 \mathrm{H}, J=19.0,9.0,2.1, H 5^{\prime}\right), 1.89$ (s, $3 \mathrm{H}, \mathrm{Me}), 1.19\left(\mathrm{~d}, 9 \mathrm{H},{ }^{2} \mathrm{~J}_{\mathrm{PH}}=10.6, \mathrm{PMe}_{3}\right) .{ }^{13} \mathrm{C}$ NMR $\left(\mathrm{CD}_{3} \mathrm{CN}, \delta\right): 201.2(\mathrm{~s}, \mathrm{C} 4), 184.1(\mathrm{~s}, \mathrm{C} 1), 146.6$ (s, PzA3), 145.9 (s, PzB3), 143.5 (s, PzC3), 140.9 (s, PzA5), 140.3 (s, PzB5), 139.9 (s, PzC5) 109.4 (s, PzB4), 109.2 (s, PzC4), 108.4 (s, PzA4), 96.9 (d, $J=2.5, C 2), 73.1\left(d,{ }^{2} J_{W P}=10.7, C 3\right), 32.9$ (s, C5 or C6), $32.9(\mathrm{~s}, \mathrm{C} 5$ or $\mathrm{C6}), 30.0(\mathrm{~s}, \mathrm{Me}), 12.9\left(\mathrm{~d},{ }^{1} \mathrm{~J}_{P C}=32.8, \mathrm{PMe}_{3}\right) .{ }^{31} \mathrm{P} \mathrm{NMR}\left(\mathrm{CDCl}_{3}, \delta\right):-0.59$ $\left(J_{\mathrm{WP}}=267\right)$. CV (MeCN): $E_{\mathrm{p}, \mathrm{a}}=+2.10 \mathrm{~V}, E_{\mathrm{p}, \mathrm{c}}=-0.67 \mathrm{~V}$. IR: $v_{\mathrm{BH}}=2510 \mathrm{~cm}^{-1}, v=1608 \mathrm{~cm}^{-1}$ (broad). ESI-MS: obs'd (\%), calc'd (\%), ppm, $\mathrm{M}^{+}: 610.1615$ (103.3), 610.1621 (86.1), 0.9; 611.1628 (81.8), 611.164 (79.5), 3.1; 612.1636 (100), 612.1644 (100), 1.3; 613.168 (54.2), 613.1688 (40.9), 0.1; 614.1680 (68.7), 614.1677 (84.7), 0.6.

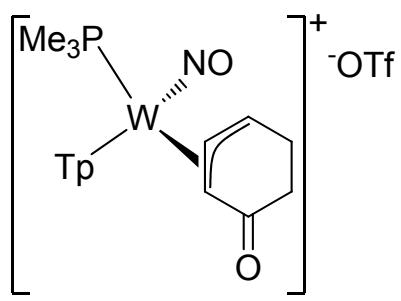

[TpW(NO)(PMe $)$ (2,3- $\eta^{2}$-(4-oxocyclohex-2-en-1-ylium)][OTf]. 20p. HOTf (0.025-0.030 g, 0.167$0.200 \mathrm{mmol}$ ) was added to a pale yellow homogeneous solution of $19 \mathrm{p}(0.015 \mathrm{~g}-0.027 \mathrm{~g}, 0.025-$ $0.045 \mathrm{mmol})$ in $\mathrm{CD}_{3} \mathrm{CN}(0.45-0.55 \mathrm{~g})$ to immediately become dark yellow-brown. The solution was transferred to an NMR tube, removed from a glovebox, frozen in $\mathrm{N}_{2(1)}$ and thawed just prior to inserting a sample into the NMR spectrometer set to $235 \mathrm{~K} .{ }^{1} \mathrm{H},{ }^{13} \mathrm{C},{ }^{31} \mathrm{P}, \mathrm{COSY}, \mathrm{NOESY}, \mathrm{HSQC}$, and $\mathrm{HMBC}$ data was collected at this temperature. Several minor species were produced in $<1: 10$ ratio to $20 \mathrm{p} .{ }^{1} \mathrm{H}$ NMR $\left(\mathrm{CD}_{3} \mathrm{CN}, \delta, 235 \mathrm{~K}\right): 8.29$ (d, $\left.J=2.0,1 \mathrm{H}, \mathrm{PzB}\right), 8.20(\mathrm{~d}, J=2.0,2 \mathrm{H}$, PzC3+PzC5), 8.10 (d, $J=2.0,1 \mathrm{H}, \mathrm{PzB} 5$ ), 8.02 (d, $J=2.0,1 \mathrm{H}, \mathrm{PzA} 5), 7.25(\mathrm{~d}, J=2.0,1 \mathrm{H}, \mathrm{PzA} 3$ ), $7.03(\mathrm{~m}, 1 \mathrm{H}, \mathrm{H} 1), 6.64(\mathrm{t}, J=2.0,1 \mathrm{H}, \mathrm{PzC} 4), 6.57(\mathrm{t}, J=2.0,1 \mathrm{H}, \mathrm{PzB} 4), 6.38(\mathrm{t}, J=2.0,1 \mathrm{H}, \mathrm{PzA} 4)$, $6.05(\mathrm{~m}, 1 \mathrm{H}, \mathrm{H} 2), 4.24(\mathrm{~d}, J=5.3,1 \mathrm{H}, \mathrm{H} 3), 3.72(\mathrm{dd}, J=21.5,10.5,1 \mathrm{H}, \mathrm{H} 6), 3.19$ (dd, $J=21.5$, $\left.10.2,1 \mathrm{H}, \mathrm{H} 6^{\prime}\right), 3.13(\mathrm{dd}, J=22.3,10.5,1 \mathrm{H}, \mathrm{H} 5), 2.61\left(\mathrm{dd}, J=22.3,10.2,4.2,1 \mathrm{H}, \mathrm{H} 5^{\prime}\right), 1.12\left(\mathrm{~d},{ }^{2} J_{\mathrm{PH}}\right.$ $\left.=10.4,9 \mathrm{H}, \mathrm{PMe}_{3}\right) .{ }^{13} \mathrm{C}$ NMR $\left(\mathrm{CD}_{3} \mathrm{CN}, \delta, 235 \mathrm{~K}\right): 209.9$ (C4), 145.1 (d, J = 2.5, PzB3), 145.0 (PzC3), 143.0 (PzA3), 140.7 (PzA5+PzB5+PzC5), 135.8 (C1), 119.9 ( $\left({ }^{1}{ }^{1} J_{\text {CF }}=320\right.$, OTf), 109.9 (PzB4), 109.4 (PzC4), 108.6 (PzA4), 93.2 (C2), 66.8 (C3), 26.3 (C5), 23.7 (C4), 11.7 (d, $\left.{ }^{1} \mathrm{JPC}_{\mathrm{PC}}=34.3, \mathrm{PMe}_{3}\right) .{ }^{31} \mathrm{P}$ $\operatorname{NMR}\left(\mathrm{CD}_{3} \mathrm{CN}, \delta, 298 \mathrm{~K}\right):-1.91\left(J_{\mathrm{WP}}=243\right)$.

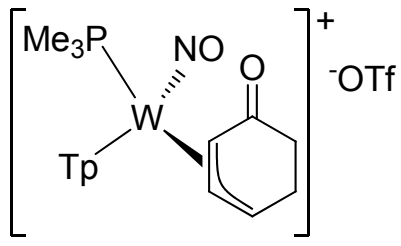


[TpW(NO)(PMe $)\left(2,3-\eta^{2}\right.$-(4-oxocyclohex-2-en-1-ylium)][OTf]. 20d (and 20p). HOTf (0.025-0.030 $\mathrm{g}, 0.167-0.200 \mathrm{mmol}$ ) was added to a pale yellow homogeneous solution of a mixture of coordination isomers of $19 \mathrm{p}, \mathrm{d}\left(2.5: 1 ; 0.020-0.028 \mathrm{~g}\right.$, 0.034-0.047 mmol) in $\mathrm{CD}_{3} \mathrm{CN}(\sim 0.5 \mathrm{~g})$ to make a dark yellow-brown solution. The solution was transferred to an NMR tube, removed from a glovebox, frozen in $\mathrm{N}_{2(1)}$ and thawed just prior to inserting a sample into the NMR spectrometer set to $235 \mathrm{~K} .{ }^{1} \mathrm{H},{ }^{13} \mathrm{C},{ }^{31} \mathrm{P}, \mathrm{COSY}, \mathrm{NOESY}, \mathrm{HSQC}$, and $\mathrm{HMBC}$ data was collected at this temperature and revealed an allylic mixture in 2.2:1 ratio. Due to multiple overlapping signals from the Major isomer, only the phenol ligand resonances of 20q are reported. ${ }^{1} \mathrm{H}$ NMR $\left(\mathrm{CD}_{3} \mathrm{CN}, \delta, 235 \mathrm{~K}\right): 6.75$ (broad, $\left.1 \mathrm{H}, \mathrm{H} 1\right), 5.66\left(\mathrm{dd},{ }^{2} J_{\mathrm{PH}}=11.0, J=5.7,1 \mathrm{H}, \mathrm{H} 2\right), 5.28(\mathrm{dd}, J=7.7$, 5.7, $1 \mathrm{H}, \mathrm{H} 3$ ), 3.70 (burried, $1 \mathrm{H}, \mathrm{H6}$ ), 3.47 (dd, $J=22.0,9.5,1 \mathrm{H}, \mathrm{H6}$ ) , 3.26 (ddd, $J=21.4,11.7$, 2.9, 1H, H5), 2.85 (dd, $\left.J=21.4,9.5,1 \mathrm{H}, \mathrm{H} 5^{\prime}\right) .{ }^{13} \mathrm{C}$ NMR $\left(\mathrm{CD}_{3} \mathrm{CN}, \delta, 235 \mathrm{~K}\right): 212.7$ (C4), $148.3(\mathrm{C} 1)$, $98.9\left(\mathrm{~d},{ }^{2} J_{\mathrm{PC}}=2.2, \mathrm{C} 2\right), 63.4\left(\mathrm{~d},{ }^{2} \mathrm{~J}_{\mathrm{PC}}=5.9, \mathrm{C} 3\right), 28.1(\mathrm{C} 5), 24.4(\mathrm{C} 6) .{ }^{31} \mathrm{P} N \mathrm{NMR}\left(\mathrm{CD}_{3} \mathrm{CN}, \delta, 298 \mathrm{~K}\right):-$ $0.37\left(J_{\mathrm{WP}}=252\right)$.

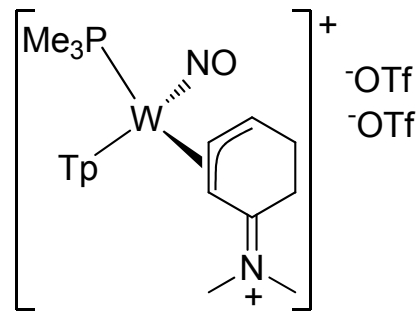

[TpW(NO)(PMe 3 )(2,3- $\eta^{2}$-(4-(dimethyliminio)cyclohex-2-en-1-ylium))](OTf) 2. 21p. A solution of HOTf $(0.023 \mathrm{~g}, 0.15 \mathrm{mmol})$ in $\mathrm{CH}_{3} \mathrm{CN}(1.07 \mathrm{~g})$, was added to a polypropylene vial containing 14 $(0.103 \mathrm{~g}, 0.13 \mathrm{mmol})$ to make a homogeneous yellow solution. After ${ }^{31} \mathrm{P} \mathrm{NMR}$ analysis confirmed the completion of the reaction, the reaction solution was transferred to vial and the solvent evaporated under reduced pressure. The yellow film was dissolved in $\mathrm{CH}_{2} \mathrm{Cl}_{2}$ and added to $50 \mathrm{~mL}$ of stirring hexanes to precipitate a pale yellow solid. The solid was dried in vacuo $(0.105 \mathrm{~g}, 0.114 \mathrm{mmol}, 85 \%)$. Note: every piece of glassware used throughout the coarse of the reaction was flame dried immediately before use and the DCM was dried by passage through basic $\mathrm{Al}_{2} \mathrm{O}_{3} .{ }^{1} \mathrm{H}$ NMR $\left(\mathrm{CD}_{3} \mathrm{CN}, \delta\right): 8.22(\mathrm{~d}, J=2.0,1 \mathrm{H}, \mathrm{Tp}), 8.20(\mathrm{~d}, J=2.0,1 \mathrm{H}, \mathrm{Tp}), 8.19(\mathrm{~d}, J=2.0$, $1 \mathrm{H}, \mathrm{Tp}), 8.09(\mathrm{~d}, J=2.0,1 \mathrm{H}, \mathrm{Tp}), 8.07(\mathrm{~d}, J=2.0,1 \mathrm{H}, \mathrm{Tp}), 7.17(\mathrm{~m}, 1 \mathrm{H}, \mathrm{H} 4), 7.12(\mathrm{~d}, J=2.0,1 \mathrm{H}$, $\mathrm{Tp}), 6.65(\mathrm{t}, J=2.0,1 \mathrm{H}, \mathrm{Tp} 4), 6.58(\mathrm{t}, J=2.0,1 \mathrm{H}, \mathrm{Tp} 4), 6.47(\mathrm{t}, J=2.0,1 \mathrm{H}, \mathrm{Tp} 4), 5.92(\mathrm{broad}, 1 \mathrm{H}$, $\mathrm{H} 3), 3.93(\mathrm{~d}, \mathrm{~J}=6.4,1 \mathrm{H}, \mathrm{H} 2), 3.69(\mathrm{~m}, 1 \mathrm{H}, \mathrm{H} 5), 3.56(\mathrm{~s}, 3 \mathrm{H}, \mathrm{N}-\mathrm{Me}), 3.32(\mathrm{~m}, 1 \mathrm{H}, \mathrm{H} 5), 2.91(\mathrm{dd}, J=$ 20.1, 9.5, 1H, H6), 2.74 (s, 3H, N-Me'), 2.47 (dd, $J=20.1,6.2,1 \mathrm{H}, \mathrm{H} 6), 1.22$ (d, J = 10.1, 9H, $\left.\mathrm{PMe}_{3}\right) .{ }^{13} \mathrm{C} \mathrm{NMR}\left(\mathrm{CD}_{3} \mathrm{CN}, \delta\right): 182.8$ (C1), 145.5 (Tp), 145.0 (Tp), 142.4 (Tp), 140.9 (Tp), 140.7 (Tp), 140.5 (Tp), 133.3 (C4), 109.8 (Tp4), 109.5 (Tp4), 109.0 (Tp4), 94.9 (C3), 62.6 (C2), 44.0 (NMe), 43.7 (NMe), $24.7(\mathrm{C} 5), 24.5(\mathrm{C} 6), 12.4\left(\mathrm{~d},{ }^{2} \mathrm{~J}_{\mathrm{PC}}=34.0, \mathrm{PMe}_{3}\right) .{ }^{31} \mathrm{P}\left(\mathrm{CD}_{3} \mathrm{CN}, \delta\right):-5.29\left(J_{\mathrm{PW}}=248\right) . \mathrm{CV}$ (MeCN): $E_{\mathrm{p}, \mathrm{a}}=+2.35 \mathrm{~V}, E_{\mathrm{p}, \mathrm{c}}=-0.30 \mathrm{~V}$. IR: $v_{\mathrm{BH}}=2506 \mathrm{~cm}^{-1}, v=1678 \mathrm{~cm}^{-1}, v=1581 \mathrm{~cm}^{-1}$ (broad). ESI-MS: Sample was too unstable to collect HRMS data. 


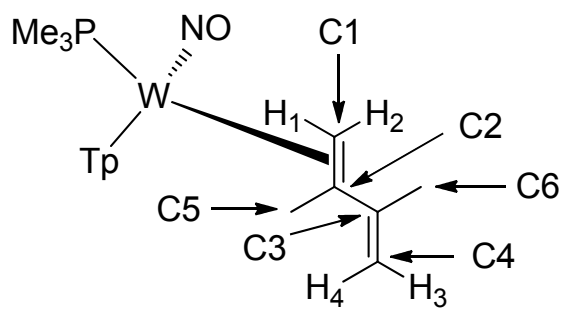

TpW(NO)(PMe $)_{3}$ (Exo-1,2- $\boldsymbol{\eta}^{2}$-2,3-dimethylbutadiene). 22 (exo-22). $\mathrm{NEt}_{3}$ (0.100 g, $0.988 \mathrm{mmol}^{\text {) }}$ was added to a heterogeneous yellow solution of $12(0.101 \mathrm{~g}, 0.137 \mathrm{mmol})$ in $\mathrm{CHCl}_{3}(6.68 \mathrm{~g})$ to make a homogeneous solution that was added to a $54{ }^{\circ} \mathrm{C}$ oil bath and was allowed to stir. After 1 hour, the yellow solution was remove from the oil bath and glovebox. The solution was diluted with $100 \mathrm{~mL} \mathrm{Et}_{2} \mathrm{O}$ and extracted with $4 \times 25 \mathrm{~mL} \mathrm{NaHCO}_{3}$ (saturated, aqueous). The water layer was back-extracted with $2 \times 25 \mathrm{~mL} \mathrm{Et}{ }_{2} \mathrm{O}$. The organic layer was dried with $\mathrm{MgSO}_{4}$, filtered through a $60 \mathrm{~mL}$ coarse porosity fritted funnel and the solvent removed to yield a pale yellow solid (0.079 g, $0.135 \mathrm{mmol}, 98 \%$ yield; uncorrected for small amount of residual DCM). ${ }^{1} \mathrm{H}$ NMR $\left(\mathrm{CDCl}_{3}, \delta\right): 8.33$ (d, J = 2.0, 1H, PzA3), 8.10 (d, J = 2.0, 1H, PzB3), 7.7 (d, J = 2.0, 1H, PzC5), 7.66 (d, $J=2.0,1 \mathrm{H}, \mathrm{PzB} 5), 7.64(\mathrm{~d}, J=2.0,1 \mathrm{H}, \mathrm{PzA} 5), 7.58(\mathrm{~d}, J=2.0,1 \mathrm{H}, \mathrm{PzC} 3), 6.25(\mathrm{t}, J=2.0,1 \mathrm{H}, \mathrm{PzB} 4)$, $6.21(\mathrm{t}, J=2.0,1 \mathrm{H}, \mathrm{PzC} 4), 6.17(\mathrm{t}, J=2.0,1 \mathrm{H}, \mathrm{PzA} 4), 4.71(\mathrm{br}(\mathrm{s}), 1 \mathrm{H}, \mathrm{H3}), 4.28(\mathrm{~d}, J=2.6,1 \mathrm{H}, \mathrm{H} 4)$, $2.55\left(\mathrm{dd},{ }^{3} J_{\mathrm{PH}}=10.3, \mathrm{~J}=5.9,1 \mathrm{H}, \mathrm{H} 2\right), 1.72(\mathrm{br}(\mathrm{s}), 3 \mathrm{H}, \mathrm{Me}-6), 1.44\left(\mathrm{dd},{ }^{3} J_{\mathrm{PH}}=9.0, \mathrm{~J}=5.9,1 \mathrm{H}, \mathrm{H} 1\right)$, $\left.1.35\left(\mathrm{~d},{ }^{2} J_{\mathrm{PH}}=8.1,9 \mathrm{H}, \mathrm{PMe} 3\right), 0.88(\mathrm{~d}, J=1.2,3 \mathrm{H}, \mathrm{Me}-5) \cdot{ }^{13} \mathrm{C} \mathrm{NMR}_{(\mathrm{CDCl}}, \delta\right): 157.1\left(\mathrm{~d},{ }^{3} J_{\mathrm{PC}}=1.5\right.$, C3), 143.4 (PzB3), 141.8 (PzC3), 141.0 (PzA3), 136.4 (PzC5), 135.8 (PzA5), 135.6 (PzB5), 106.9 (C4), 106.0 (PzB4), 105.7 (PzA4), $105.6(\mathrm{PzC} 4), 60.8(\mathrm{C} 2), 50.2\left(\mathrm{~d},{ }^{2} J_{\mathrm{PC}}=11.0, \mathrm{C} 1\right), 24.0\left(\mathrm{~d},{ }^{3} J_{P C}=\right.$ 1.7, C5), $21.2(C 6), 13.7\left(d,{ }^{1} J_{P C}=27.7, P M e 3\right) .{ }^{31} \mathrm{P} \mathrm{NMR}\left(\mathrm{CDCl}_{3}, \delta\right):-13.95\left(J_{\mathrm{WP}}=266\right) . \mathrm{IR}: \mathrm{v}_{\mathrm{BH}}=$ $2482 \mathrm{~cm}^{-1}, \mathrm{v}=1592 \mathrm{~cm}^{-1}, \mathrm{v}_{\mathrm{NO}}=1545 \mathrm{~cm}^{-1} . \mathrm{CV}(\mathrm{MeCN}): E_{\mathrm{p}, \mathrm{a}}=+0.37 \mathrm{~V}$. ESI-MS: obs'd (\%), calc'd (\%), ppm, (M+H) ${ }^{+}: 584.1828$ (93.4), 584.1828 (86.8), 0.0; 585.1861 (94.3), 585.1854 (79.3), 1.1; 586.185 (100), 586.1852 (100), 0.3; 587.1899 (47.6), 587.1896 (40.1), 0.5; 588.1888 (108.8), 588.1884 (84.9), 0.5 .

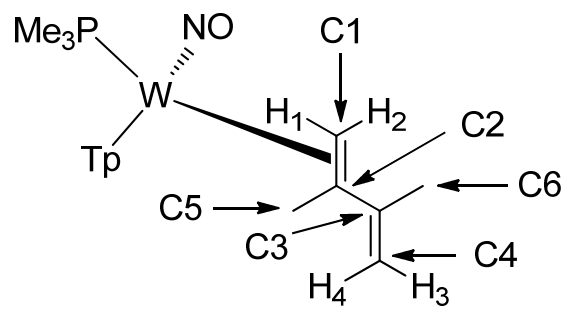

Exo

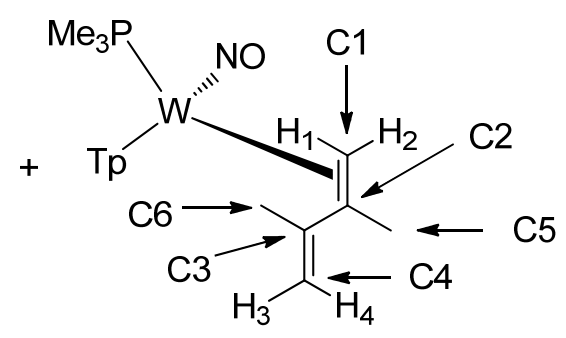

Endo

$\mathrm{TpW}(\mathrm{NO})\left(\mathrm{PMe}_{3}\right)\left(1,2-\eta^{2}-2,3-d i m e t h y l b u t a d i e n e\right)$. Endo-22, Exo-22. Isolated Endo:Exo ratio 1.6:1. Butadiene $(0.200 \mathrm{~g}, 2.44 \mathrm{mmol})$ was added to an oven-dried test tube containing a heterogeneous solution of $\mathrm{TpW}(\mathrm{NO})\left(\mathrm{PMe}_{3}\right)\left(\eta^{2}\right.$-benzene) $(0.105 \mathrm{~g}, 0.181 \mathrm{mmol})$ and DME (1.51 g). The solution was added to a $54{ }^{\circ} \mathrm{C}$ oil bath and allowed to stir for $1 \mathrm{~h} 40 \mathrm{~min}$. The solution was removed from the oil bath and diluted with $40 \mathrm{~mL}$ hexanes in a $125 \mathrm{~mL}$ filter flask to precipitate a small amount of material. As the solvent was removed and the solution cooled 
down, some brown precipitate formed. The solvent was removed completely. The residue was redissolved in $1 \mathrm{~mL} \mathrm{DCM}$ and $25 \mathrm{mLEt} \mathrm{E}_{2} \mathrm{O}$ was added to the solution followed by the addition of $25 \mathrm{~mL}$ of hexanes. The solution was cooled via evaporation for $\sim 5$ minutes and a brown precipitate formed and was collected on a $15 \mathrm{~mL}$ fine-porosity fritted funnel and was discarded. The filtrate solvent was removed in vacuo, the residue was transferred to a vial tared vial with $\mathrm{DCM}$, andthe solvent was removed in vacuo to produce a light brown solid $(0.084 \mathrm{~g}, 0.144$ mmol, $79 \%$ yield). ${ }^{1} \mathrm{H}$ NMR (CDCl $3, \delta$ ): Endo: $8.11(\mathrm{~d}, J=2.0,1 \mathrm{H}, \mathrm{PzA} 3), 8.03(\mathrm{~d}, J=2.0,1 \mathrm{H}$, PzB3), 7.64 (d, $J=2.0,2 \mathrm{H}, \mathrm{Tp} 5+\mathrm{Tp} 5), 7.62$ (d, J=2.0, 1H, Tp5), 7.44 (d, J=2.0, 1H, PzC3), 6.23 (t, $J=2.0,1 \mathrm{H}, \mathrm{PzB} 4), 6.18(\mathrm{t}, J=2.0,1 \mathrm{H}, \mathrm{PzA} 4), 6.12(\mathrm{t}, J=2.0,1 \mathrm{H}, \mathrm{PzC} 4), 4.52(\mathrm{~d}, J=2.5,1 \mathrm{H}, \mathrm{H} 4)$, $4.14(\mathrm{dd}, J=2.5,1.0,1 \mathrm{H}, \mathrm{H} 3), 2.28(\mathrm{~s}, 3 \mathrm{H}, \mathrm{Me}-5), 2.15\left(\mathrm{dd},{ }^{3} \mathrm{JPH}_{\mathrm{PH}}=11.1, J=5.4,1 \mathrm{H}, \mathrm{H} 1\right), 1.92(\mathrm{dd}$, $\left.3_{\mathrm{PH}}=8.7, J=5.4,1 \mathrm{H}, \mathrm{H} 2\right), 1.34\left(\mathrm{~d},{ }^{2} \mathrm{JPH}_{\mathrm{PH}}=8.1,9 \mathrm{H}, \mathrm{PMe}_{3}\right), 0.45(\mathrm{~d}, J=1.0,3 \mathrm{H}, \mathrm{Me}-6) .{ }^{13} \mathrm{C} \mathrm{NMR}$ $\left(\mathrm{CDCl}_{3}, \delta\right)$ : Endo: 155.1 (d, $\left.{ }^{3} J_{\mathrm{PC}}=2.4, \mathrm{C} 3\right), 143.7(\mathrm{~d}, J=1.6, \mathrm{PzB} 3), 143.3$ (PzA3), 142.6 (PzC3), 136.1 (Tp5), 135.8 (Tp5), 135.6 (Tp5), 108.1 (C4), 105.9/105.9 (PzA4/PzB4), 105.2 (PzC4), 60.9 (C2), $48.8\left(\mathrm{~d},{ }^{2} \mathrm{JPC}_{\mathrm{PC}}=11.1, \mathrm{C} 1\right), 29.9(\mathrm{C} 5), 21.1(\mathrm{C} 6), 13.9\left(\mathrm{~d},{ }^{1} \mathrm{~J}_{\mathrm{PC}}=27.8, \mathrm{PMe}_{3}\right) .{ }^{31} \mathrm{P} \mathrm{NMR}\left(\mathrm{CDCl}_{3}, \delta\right)$ : $-14.29\left(J_{\mathrm{WP}}=267 \mathrm{~Hz}\right)$. The exo isomer was characterized independently. IR: $v_{\mathrm{BH}}=2482 \mathrm{~cm}^{-1}, v=$ $1592 \mathrm{~cm}^{-1}, v_{\mathrm{NO}}=1545 \mathrm{~cm}^{-1} . \mathrm{CV}(\mathrm{MeCN}): E_{\mathrm{p}, \mathrm{a}}=+0.38 \mathrm{~V}$. ESI-MS: obs'd (\%), calc'd (\%), ppm, $(\mathrm{M}+\mathrm{H})^{+}: 584.1836$ (104.7), 584.1828 (86.8), 1.4; 585.1866 (69.4), 585.1854 (79.3), 1.9; 586.1877 (100), 586.1852 (100), 4.3; 587.1916 (42.1), 587.1896 (40.1), 3.5; 588.1899 (94.6), 588.1884 (84.9), 2.5 .

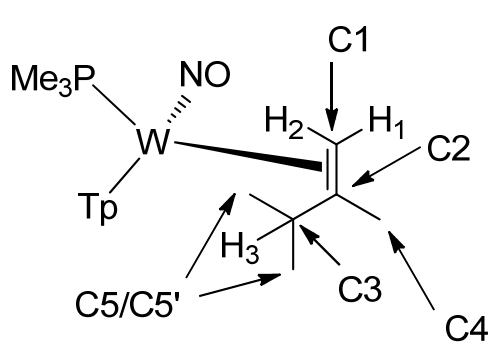

Endo (Major)

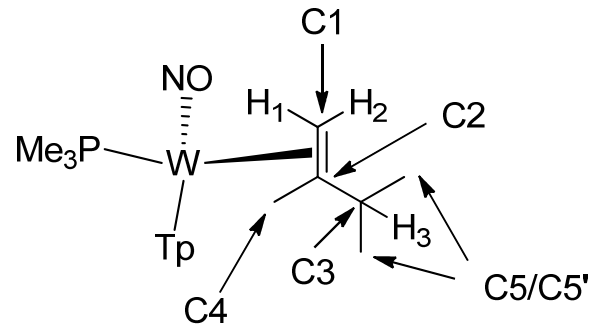

Exo (Minor)

$2.7: 1$

$\mathrm{TpW}(\mathrm{NO})\left(\mathrm{PMe}_{3}\right)\left(1,2-\eta^{2}-2,3\right.$-dimethylbut-1-ene). endo-23, exo-23. $\mathrm{NaBH}_{4}$ reduction of 12. $\mathrm{MeOH}(0.43 \mathrm{~g})$ was added to a vial containing $\mathrm{NaBH}_{4}(0.028 \mathrm{~g}, 0.740 \mathrm{mmol})$ and the heterogeneous solution quickly transferred to a vial containing a bright yellow solution of $\mathbf{1 2}$ $(0.021 \mathrm{~g}, 0.029 \mathrm{mmol})$ in $\mathrm{MeOH}(0.43 \mathrm{~g})$. Vigorous effervescence occurred and the solution color becoming pale yellow. After 20 minutes, the reaction solution was removed from the glovebox, diluted with $\mathrm{DCM}(15 \mathrm{~mL}$ ), extracted with $3 \times 15 \mathrm{~mL} \mathrm{NaHCO}$ (saturated, aqueous), back-extracted with $2 \times 15 \mathrm{~mL} \mathrm{DCM}$, dried with $\mathrm{MgSO}_{4}$, filtered through a $60 \mathrm{~mL}$ coarse-porosity fritted funnel, and the filtrate solvent removed in vacuo. The residue was transferred to a tared vial with $4 \times 2 \mathrm{~mL} D C M$, and upon evaporation of the solvent, a thin flaky tan solid was produced (0.012 g, $0.020 \mathrm{mmol}, 72$ \% yield; 2.7:1 Endo-23:Exo-23). ${ }^{1} \mathrm{H}$ NMR $\left(\mathrm{CDCl}_{3}, \delta\right)$ : Endo isomer: 8.20 (d, $J=2.0,1 \mathrm{H}$, PzA3), 7.95 (d, $J=2.0,1 \mathrm{H}$, PzB3), 7.69 (d, $J=2.0,1 \mathrm{H}$, PzC5), 7.67/7.63 (d+d, $J=$ 2.0, 1+1H, PzA5/PzB5), $7.42(\mathrm{~d}, J=2.0,1 \mathrm{H}, \mathrm{PzC} 3), 6.2(\mathrm{t}+\mathrm{t}, J=2.0,1+1 \mathrm{H}, \mathrm{PzA} 4 / \mathrm{PzB} 4), 6.18(\mathrm{t}, J=$ 
2.0, 1H, PzC4), 2.06 (s, 3H, Me-4), $1.98\left(\mathrm{dd},{ }^{3} J_{\mathrm{PH}}=10.0, J=4.9,1 \mathrm{H}, \mathrm{H} 1\right), 1.49\left(\mathrm{dd},{ }^{3} J_{\mathrm{PH}}=10.7, J=\right.$ 4.9, $1 \mathrm{H}, \mathrm{H} 2$ ), $1.33\left(\mathrm{~d},{ }^{2} J_{\mathrm{PH}}=7.9,9 \mathrm{H}, \mathrm{PMe}_{3}\right), 0.98(\mathrm{~d}, J=6.9,3 \mathrm{H}, \mathrm{Me}-5), 0.87$ (septet, $J=6.9,1 \mathrm{H}$, $\mathrm{H} 2$ ), $-0.23\left(\mathrm{~d}, J=6.9,3 \mathrm{H}, \mathrm{Me}-5^{\prime}\right)$, Selected minor isomer (Exo) signals:, $8.37(\mathrm{~d}, J=2.0,1 \mathrm{H}, \mathrm{PzA} 3$ ), $8.2(\mathrm{~d}, J=2.0,1 \mathrm{H}, \mathrm{PzB} 3), 7.67$ (d, $J=2.0,1 \mathrm{H}, \mathrm{PzB} 5), 7.58$ (d, $J=2.0,1+1 \mathrm{H}, \mathrm{PzA} 5 / \mathrm{PzC} 5), 6.26$ (t, $J$ $=2.0,1 \mathrm{H}, \mathrm{PzB} 4), 2.12(\mathrm{~m}, 1 \mathrm{H}, \mathrm{H} 3), 2.10\left(\mathrm{dd},{ }^{3} J_{\mathrm{PH}}=12.1, J=5.2,1 \mathrm{H}, \mathrm{H} 1\right.$ orH2), $1.35(\mathrm{~d}, J=6.9,3 \mathrm{H}$, Me-5), 1.29 (dd, $\left.{ }^{3} J_{\mathrm{PH}}=8.3, J=5.3,1 \mathrm{H}, \mathrm{H} 1 \mathrm{orH} 2\right), 1.24\left({ }^{2} J_{\mathrm{PH}}=8.3,9 \mathrm{H}, \mathrm{PMe}_{3}\right), 1.16(\mathrm{~d}, J=6.9,3 \mathrm{H}$, Me-5'), 1.01 (s, 2H, Me-4). $\left.{ }^{13} \mathrm{C} \mathrm{NMR} \mathrm{(} \mathrm{CDCl}_{3}, \delta\right)$ : Endo Isomer (Major): 143.6 (PzA3orPzB3), 143.5 (d, J = 1.8, PzA3orPzB3), 141.6 (PzC3), 136 (Tp5), 135.9 (Tp5), 135.3 (Tp5), 105.6 (Tp4+Tp4), 105.3 (Tp4), $60.7(\mathrm{C} 2), 50.0\left(\mathrm{~d},{ }^{2} J_{P C}=11.2, \mathrm{C} 1\right), 37$ (d, $\left.J=1.9, \mathrm{C} 3\right), 26.5(\mathrm{C} 5), 21.6$ (d, $\left.J=1.4, \mathrm{C} 4\right)$, $18.4\left(C^{\prime}\right), 13.9\left(d,{ }^{1} J_{P C}=27.5, P_{3}\right)$, Exo Isomer (Minor): 145.5 (PzA3), 143.4 (d, J = 1.5, PzB3), 141.8 (PzC3), 136.2 (Tp5), 135.3 (Tp5), 135.1 (Tp5), 105.9 (Tp4), 105.3 (Tp4), , 105.2 (Tp4), 62.3 $(C 2), 50.5\left(d,{ }^{2} J_{P C}=11.0, C 1\right), 41.8(C 3), 25.2(C 5), 24.2(C 5), 20.1(d, J=2.1, C 4), 13.1\left(d,{ }^{2} J_{P C}=\right.$ 27.9, $\mathrm{PMe}_{3}$ ). ${ }^{31} \mathrm{P} \mathrm{NMR}\left(\mathrm{CDCl}_{3}, \delta\right):-13.42$ (JPP $=264$; exo), -14.48 (JWP $=261$; Endo). CV (MeCN): $E_{\mathrm{p}, \mathrm{a}}=+0.24 \mathrm{~V}$. When the scan rate is increased to $>200 \mathrm{mV} / \mathrm{s}$ the $E_{\mathrm{p}, \mathrm{a}}$ become an $E_{1 / 2}=+0.20 \mathrm{~V}$. IR: $v_{\mathrm{BH}}=2485 \mathrm{~cm}^{-1}, v_{\mathrm{NO}}=1540 \mathrm{~cm}^{-1}$. ESI-MS: obs'd (\%), calc'd (\%), ppm, $(\mathrm{M}+\mathrm{H})^{+}: 586.2002$ (106.7), 586.1985 (86.8), 2.9; 587.2021 (79.3), 587.2011 (79.3), 1.8; 588.1998 (100), 588.2008 (100), 1.8; 589.2059 (41.8), 589.2052 (40.1), 1.1; 590.2064 (99.2), 590.2041 (84.9), 3.9.
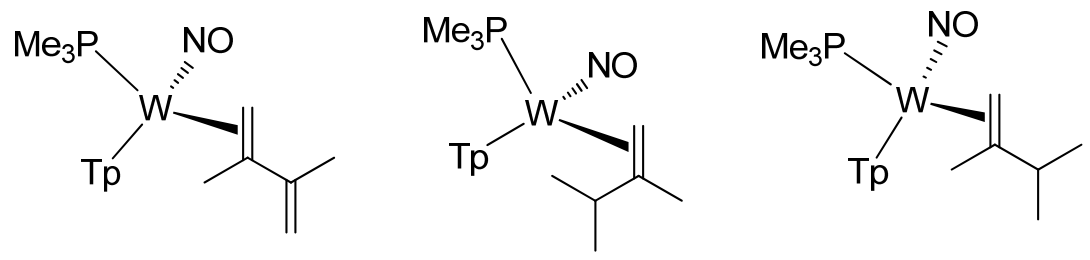

$\mathrm{Na} / \mathrm{Hg}$ amalgam reduction of $12 \mathrm{Na} / \mathrm{Hg}(1 \% ; 4.01 \mathrm{~g}, 1.740 \mathrm{mmol})$ was added to a yellow heterogeneous solution of $12(0.022 \mathrm{~g}, 0.030 \mathrm{mmol})$ and DME $(6.41 \mathrm{~g})$. The solution was allowed to stir and within 2 minutes the solution had become purple. After $2.25 \mathrm{~h}$, the murky purple solution was filtered through a pipette containing celite and the filtrate solvent removed in vacuo. The crude residue was dissolved in $\mathrm{CDCl}_{3}$ to produce a pale yellow solution and left a white solid in the evaporating flask. NMR: Three isomers are present in a 2.6:1:1.1 ratio (exo23:exo-22:endo-23). 
3, ${ }^{1} \mathrm{H}, \mathrm{CD}_{3} \mathrm{CN}$

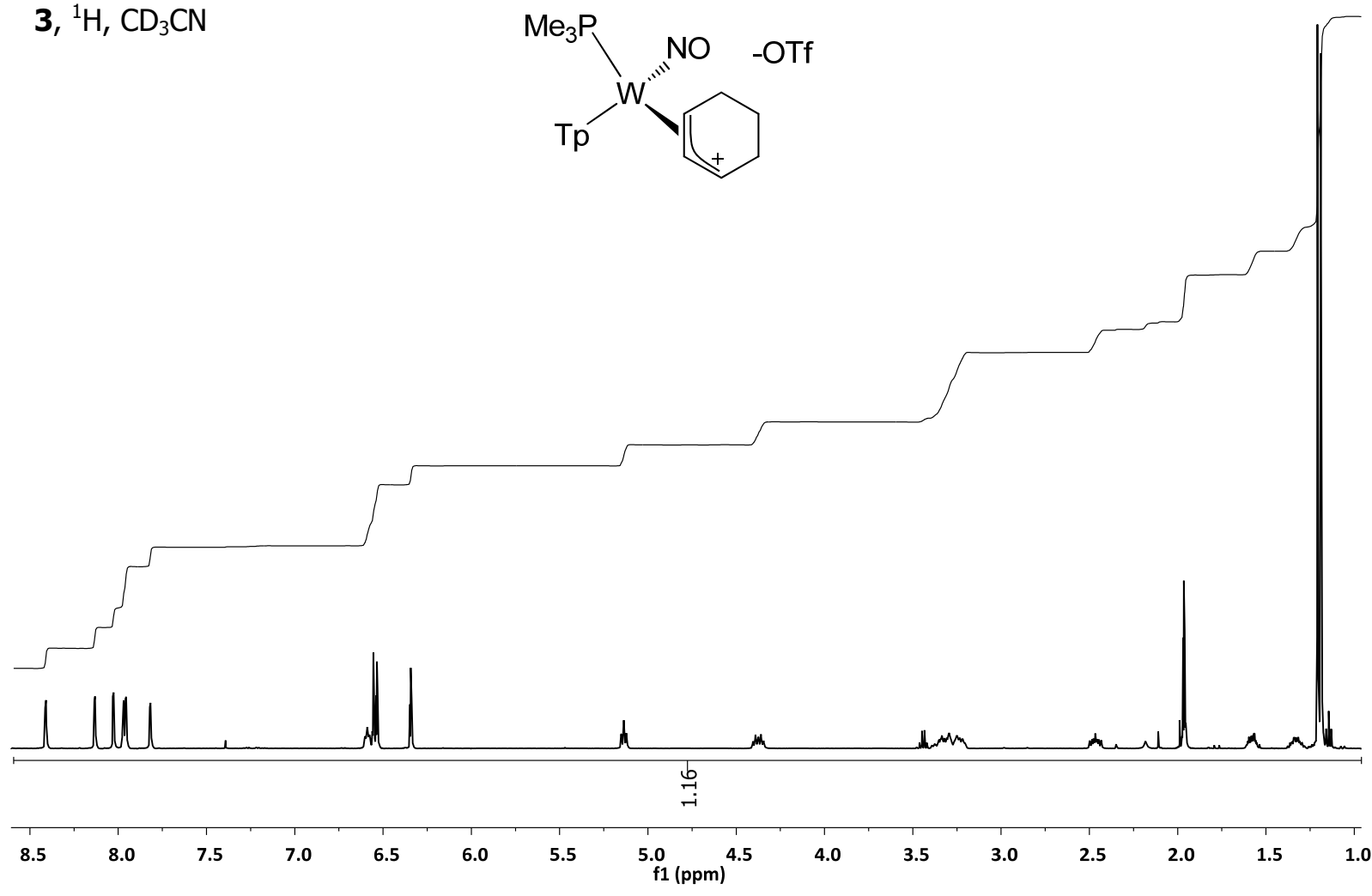

3, ${ }^{13} \mathrm{C}, \mathrm{CD}_{3} \mathrm{CN}$

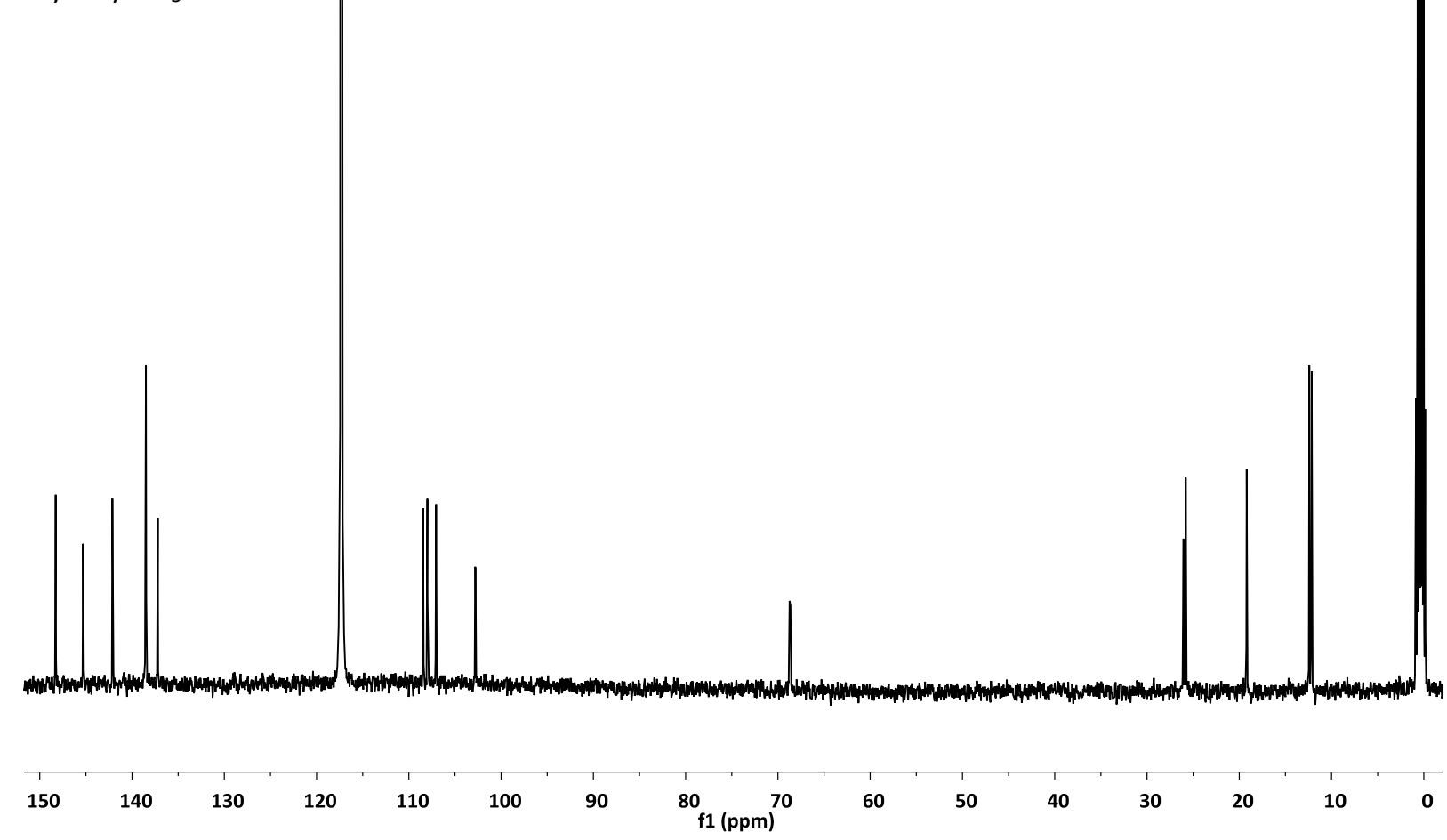




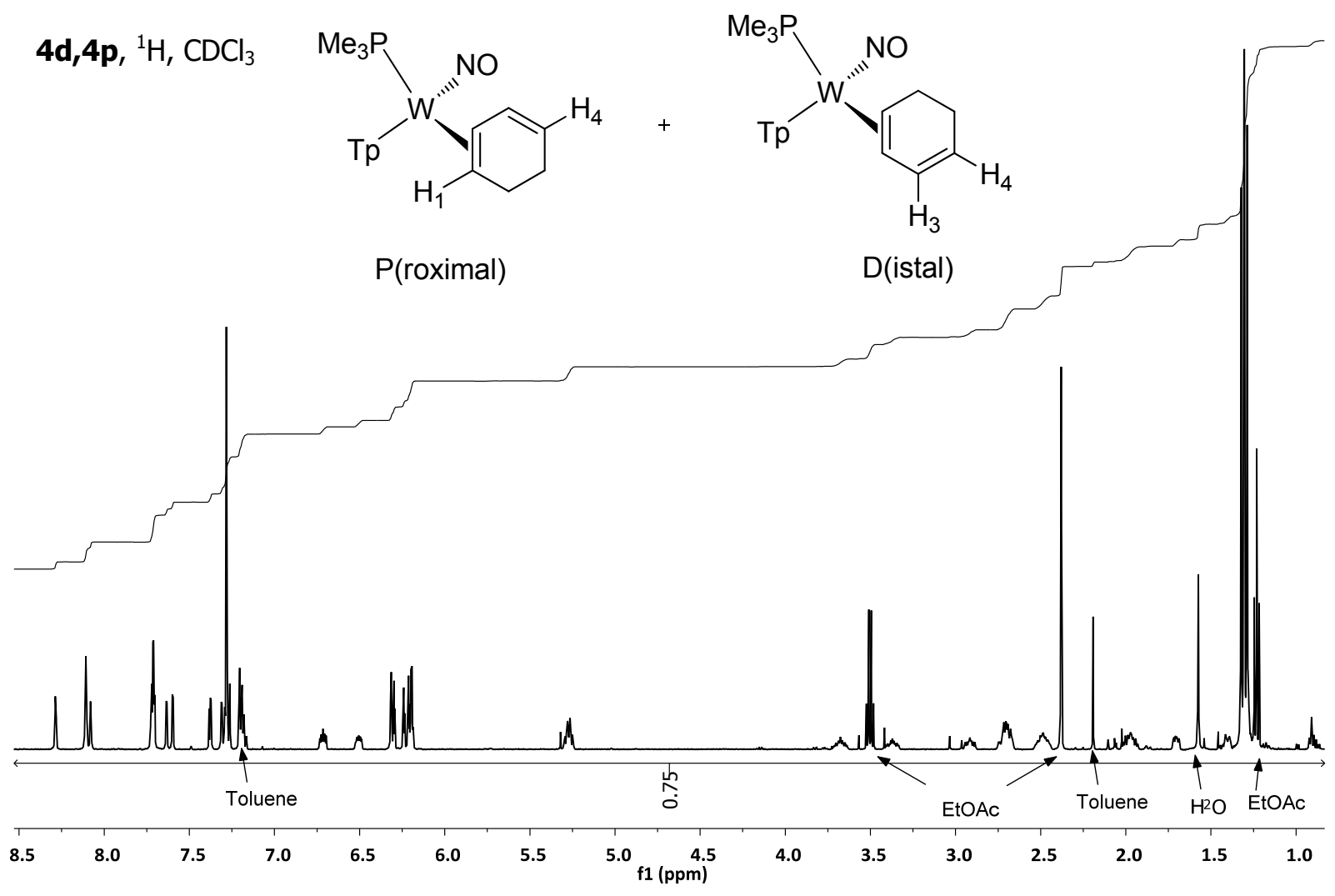

4d, $\mathbf{4 p},{ }^{13} \mathrm{C}, \mathrm{CDCl}_{3}$

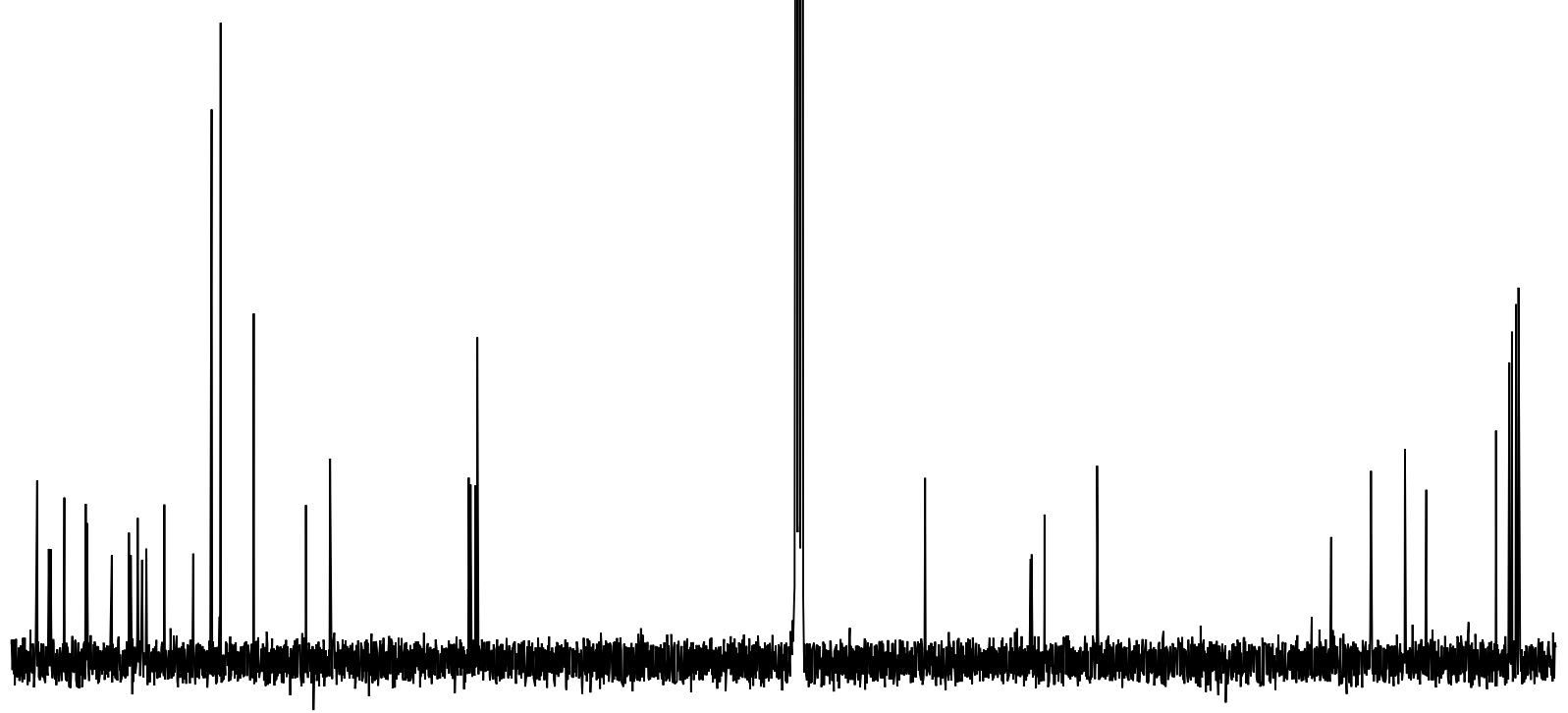

125 115 105 95 $8580 \quad 75$ 
4d, ${ }^{1} \mathrm{H}, \mathrm{CDCl}_{3}$

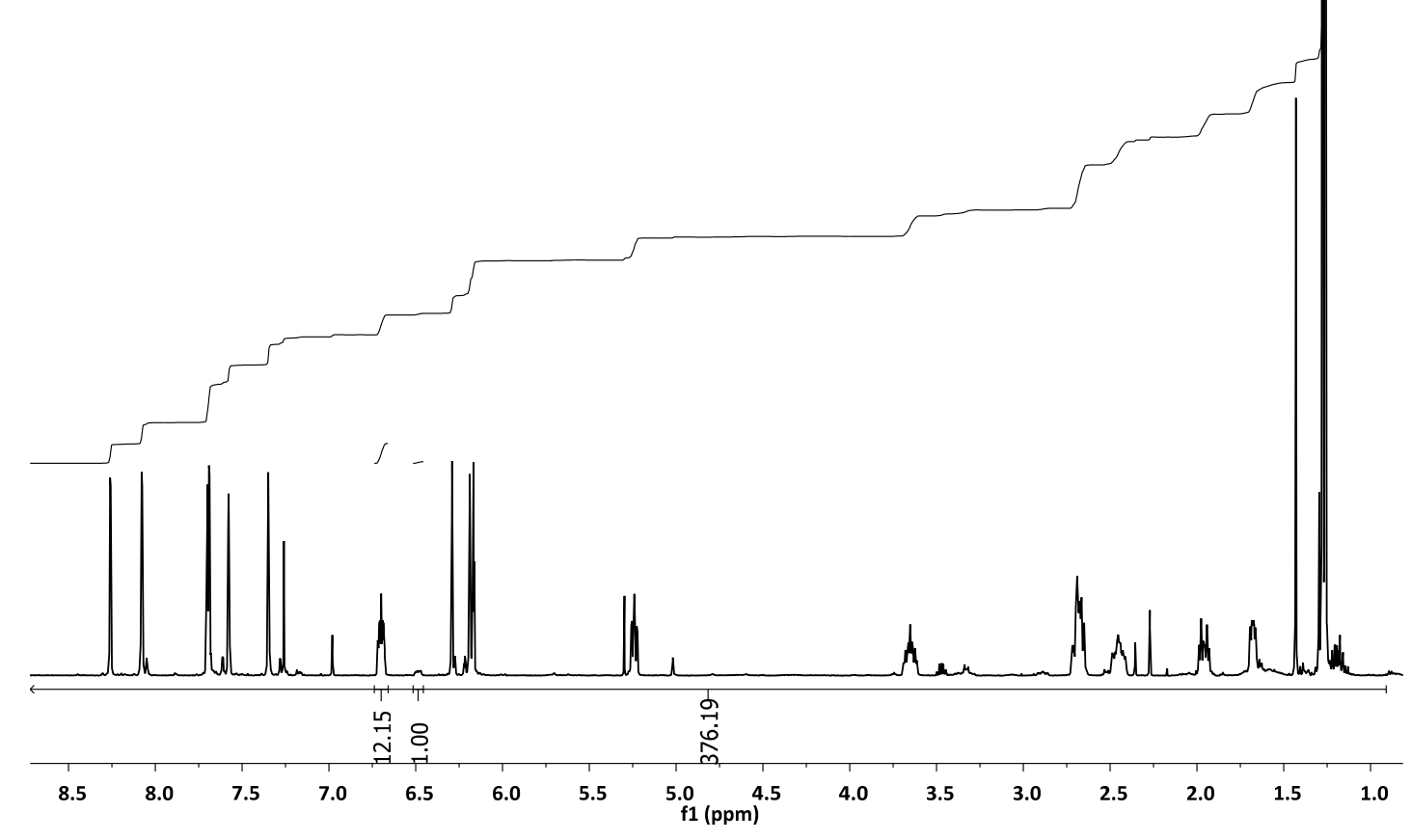




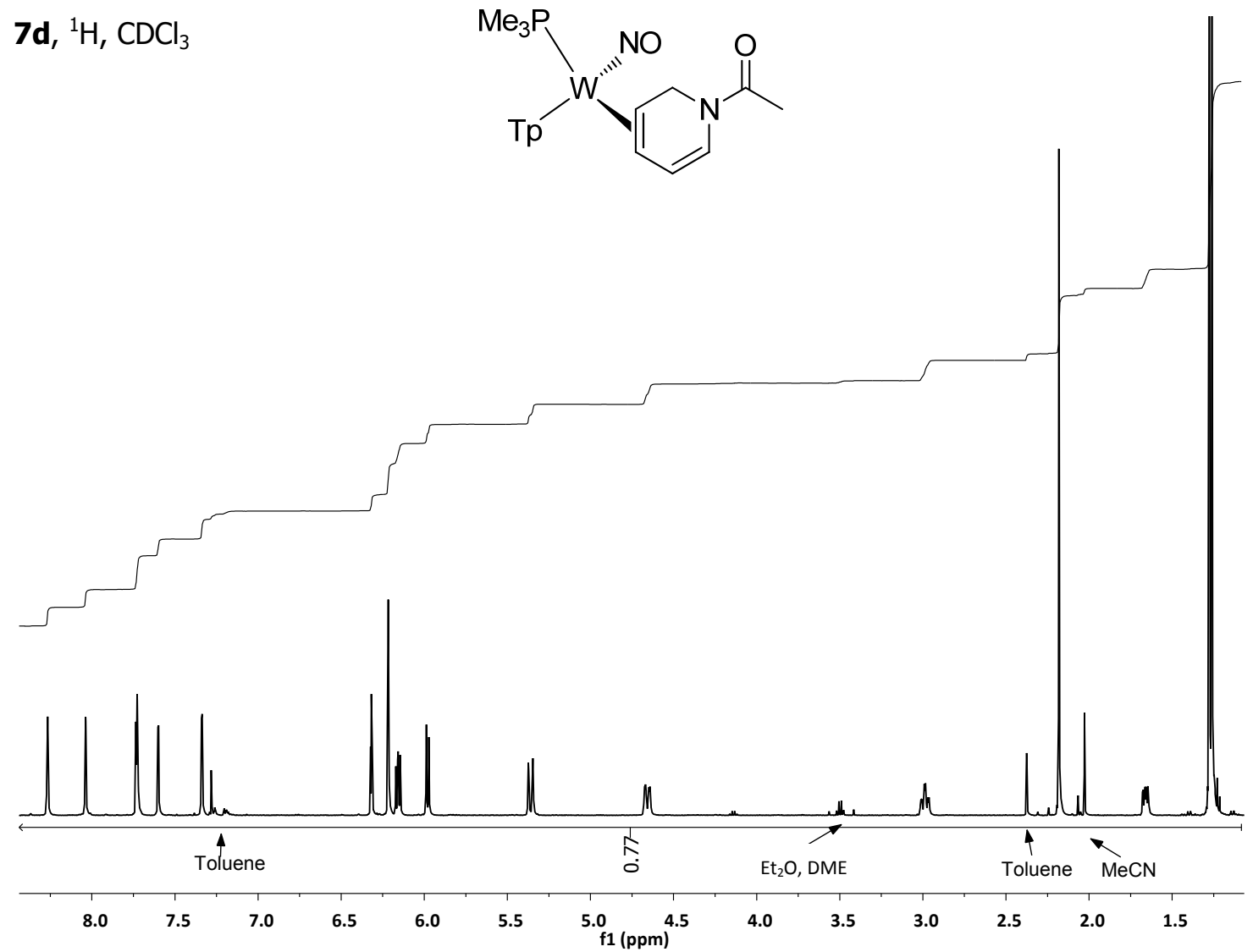

7d, ${ }^{13} \mathrm{C}, \mathrm{CDCl}_{3}$

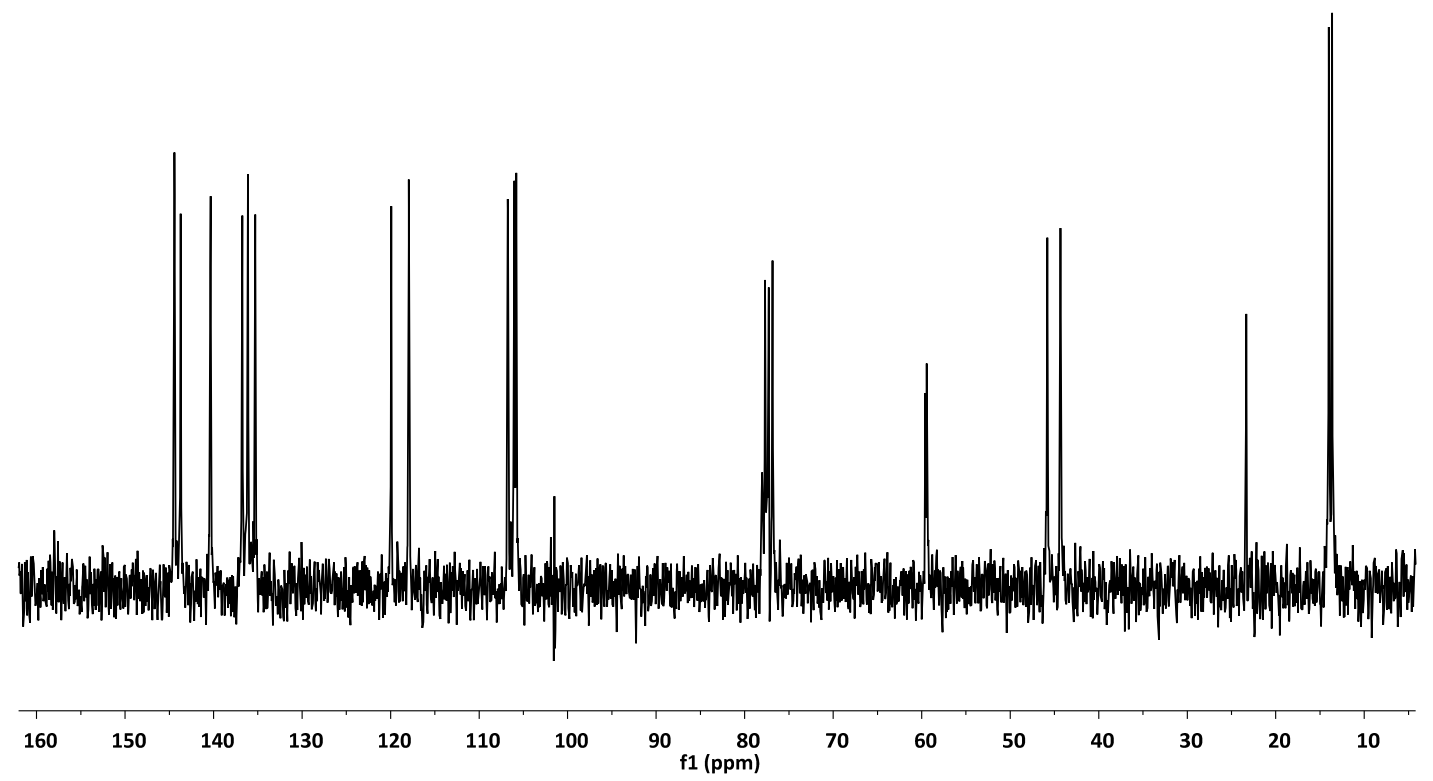


8c, ${ }^{1} \mathrm{H}, \mathrm{CDCl}_{3}$

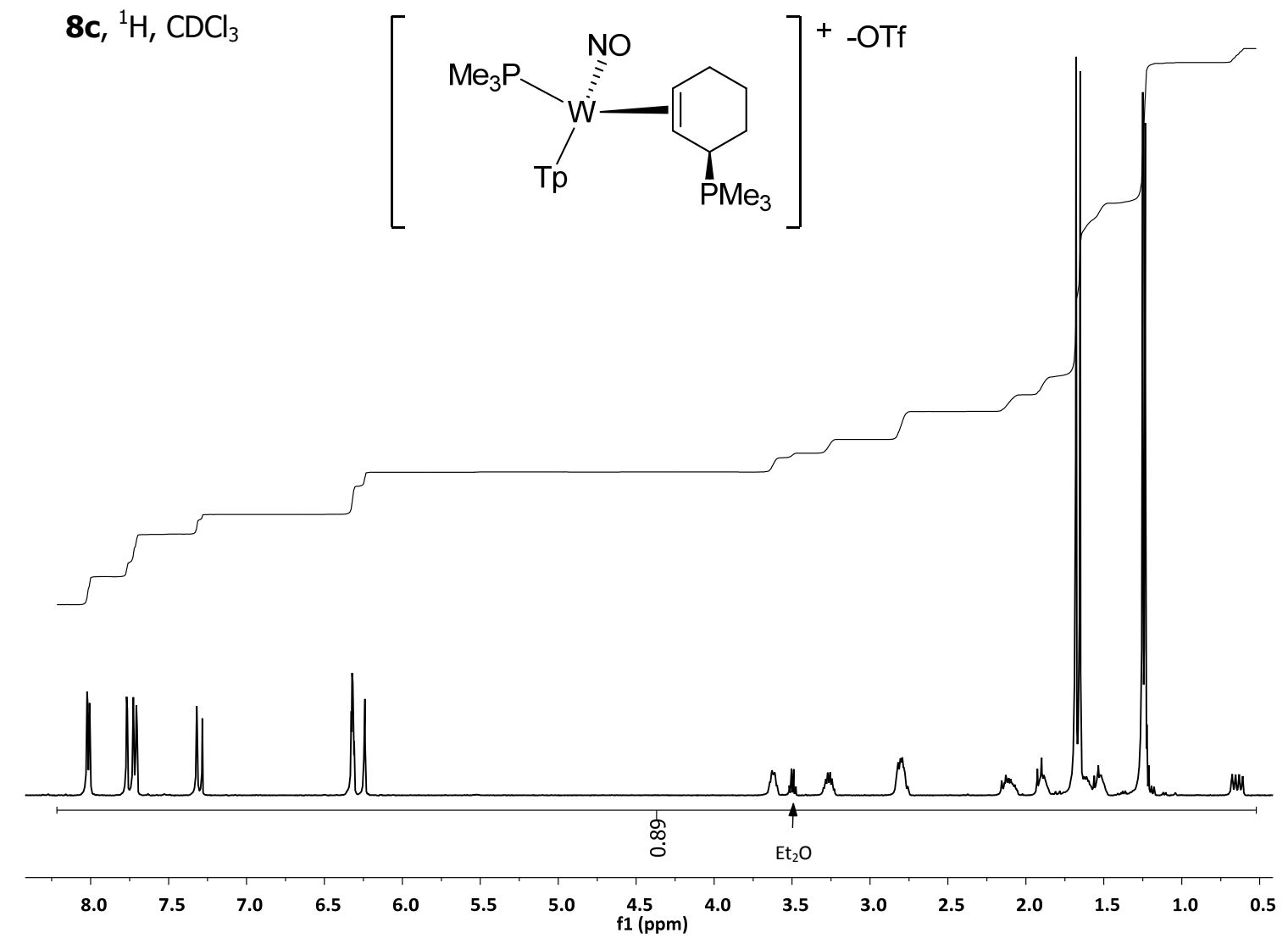

8c, ${ }^{13} \mathrm{C}_{,} \mathrm{CDCl}_{3}$

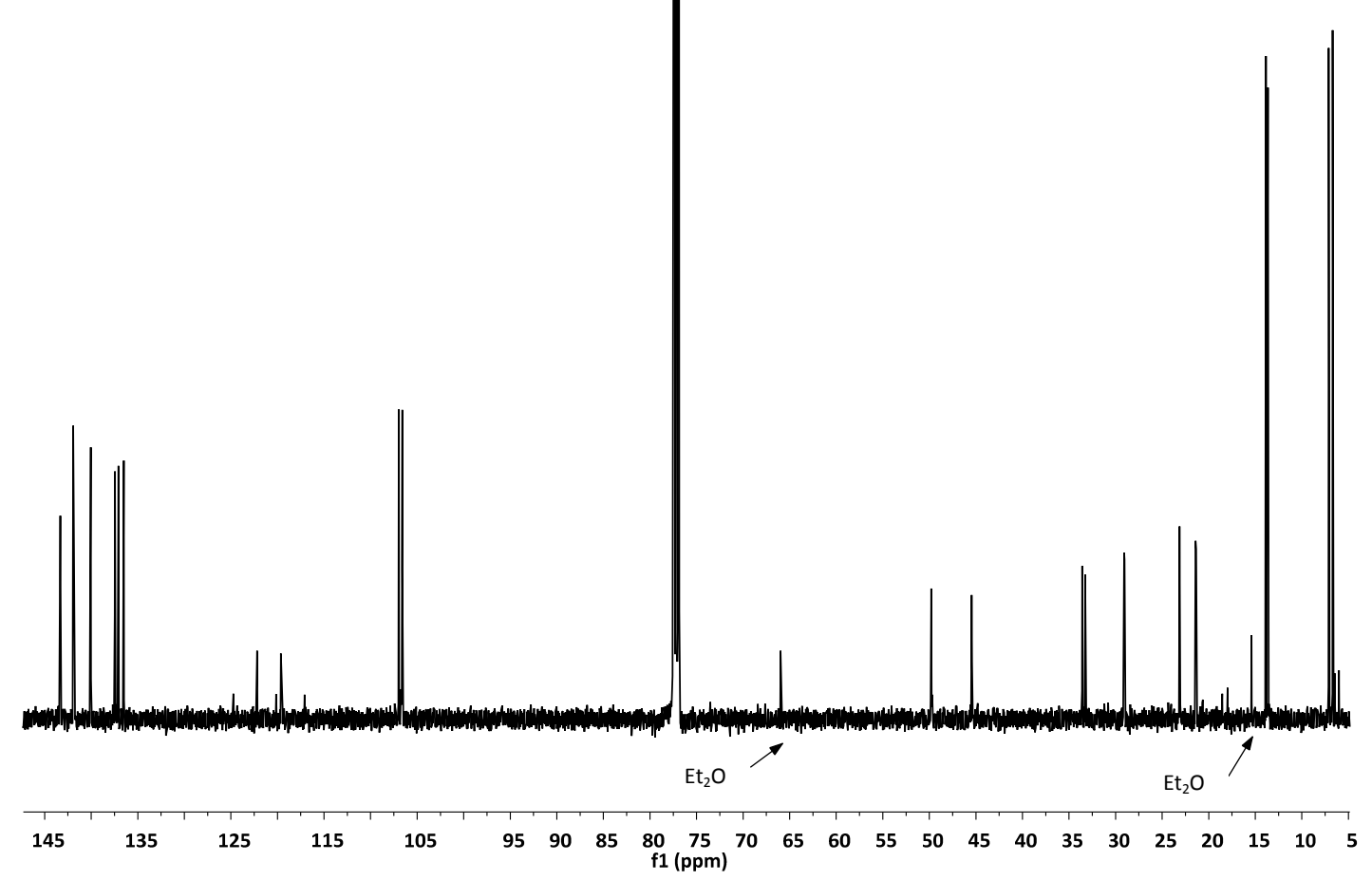




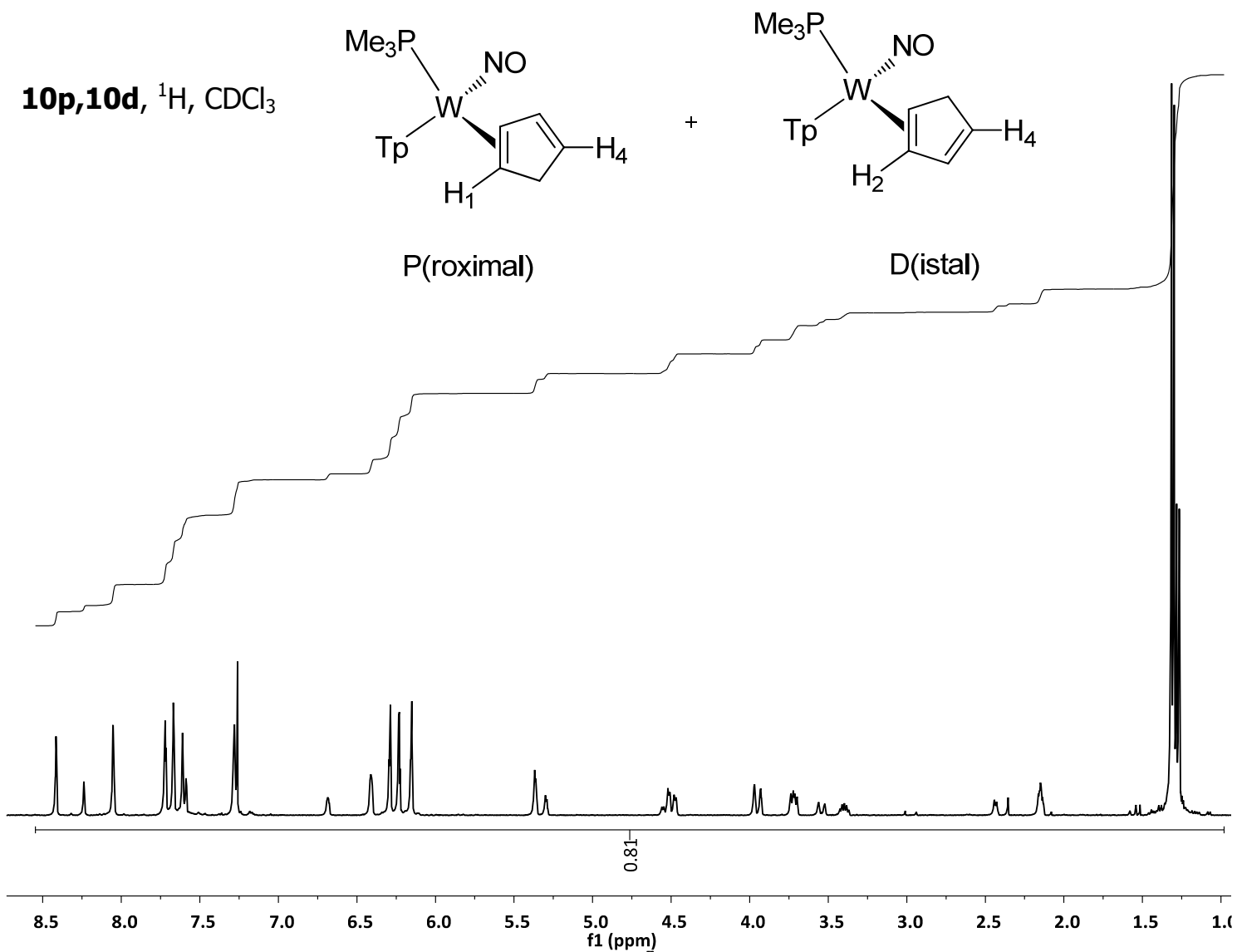

10p, 10d, ${ }^{13} \mathrm{C}, \mathrm{CDCl}_{3}$
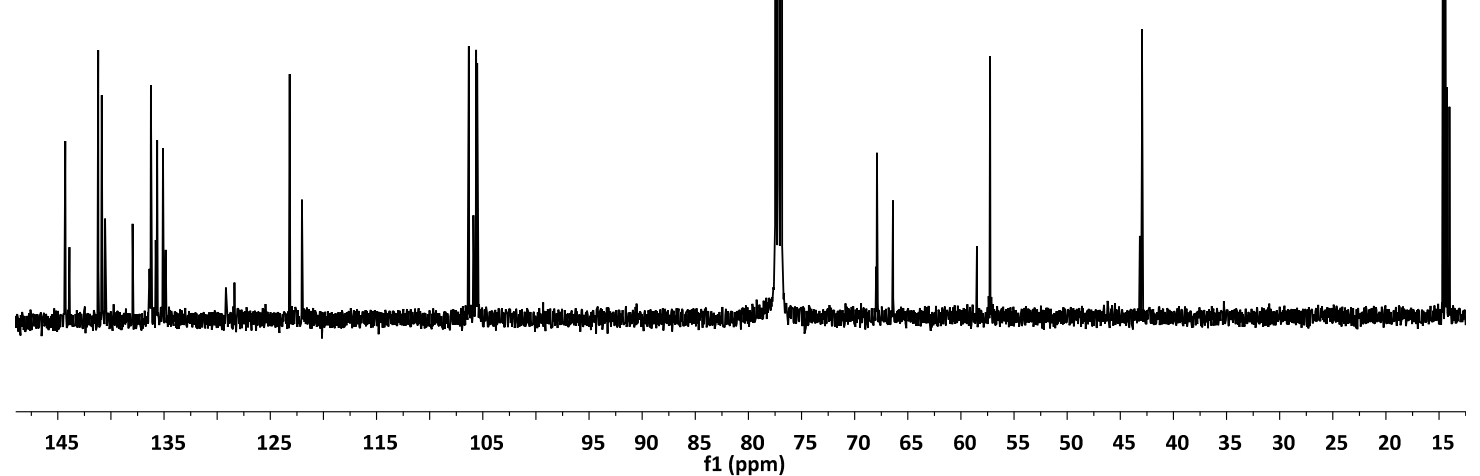
Exo-11,Endo-11, ${ }^{1} \mathrm{H}, \mathrm{CD}_{3} \mathrm{CN}$
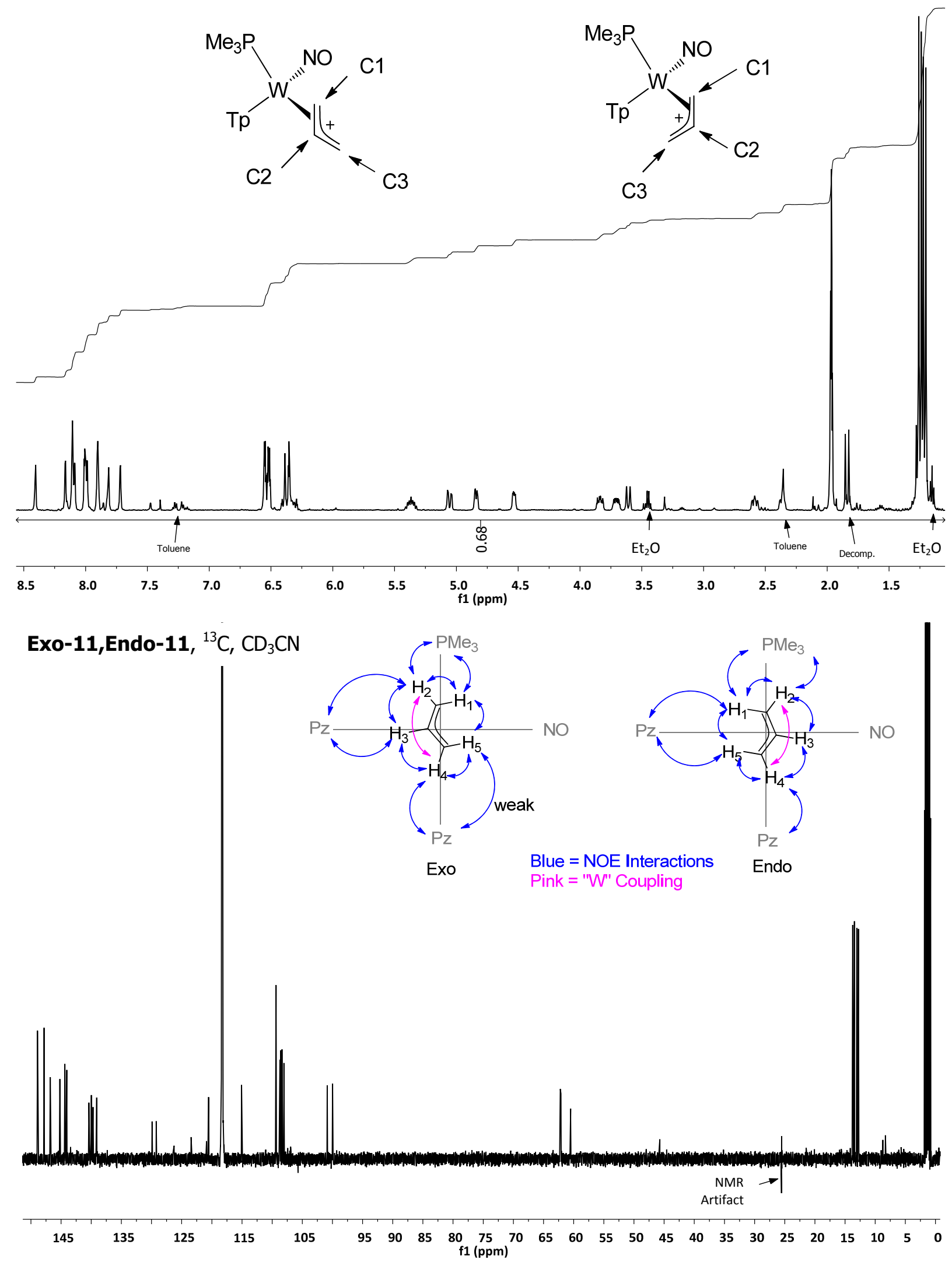
Exo-12,Endo-12, ${ }^{1} \mathrm{H}, \mathrm{CD}_{3} \mathrm{CN}$

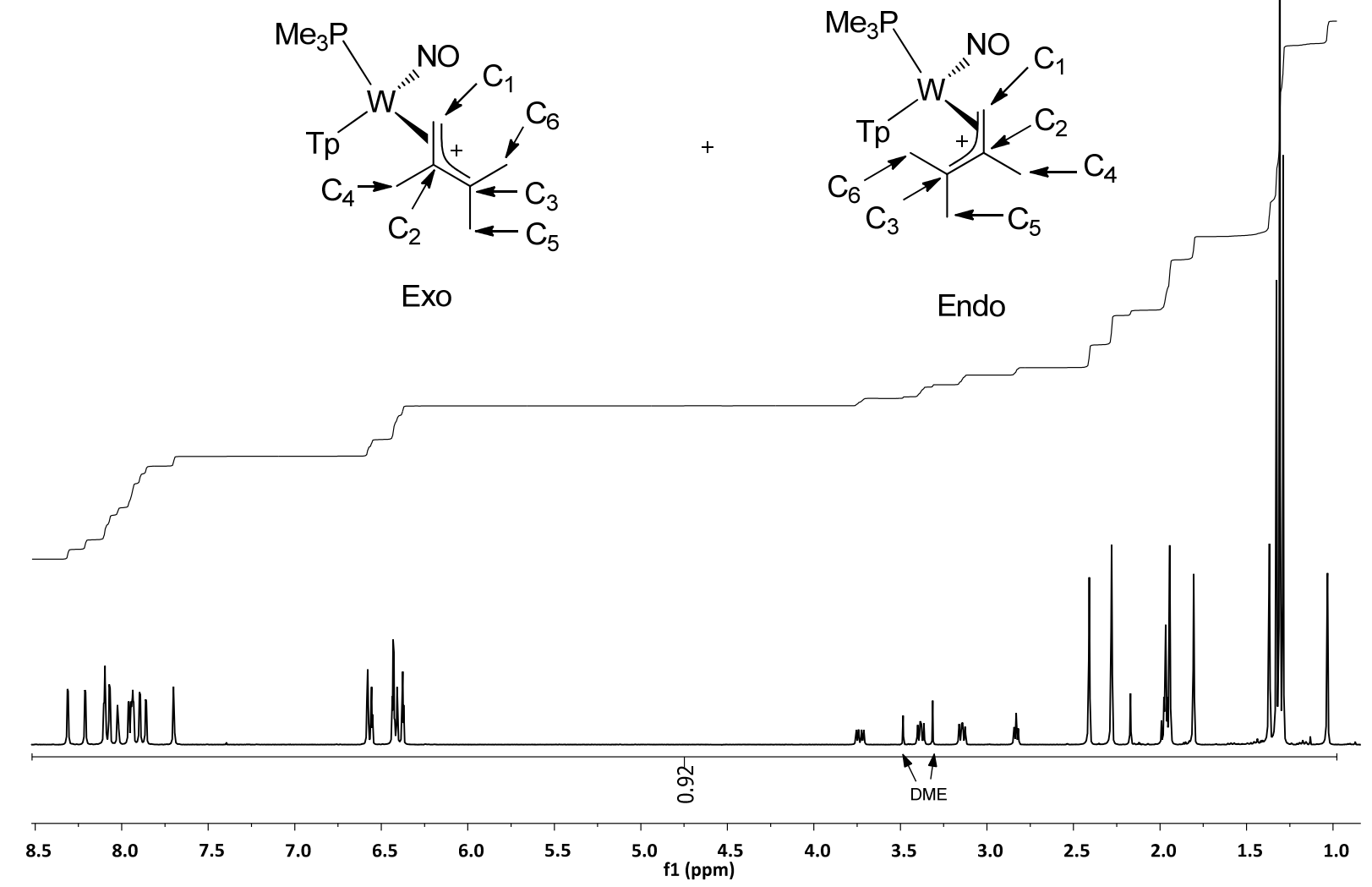

Exo-12,Endo-12, ${ }^{13} \mathrm{C}, \mathrm{CD}_{3} \mathrm{CN}$
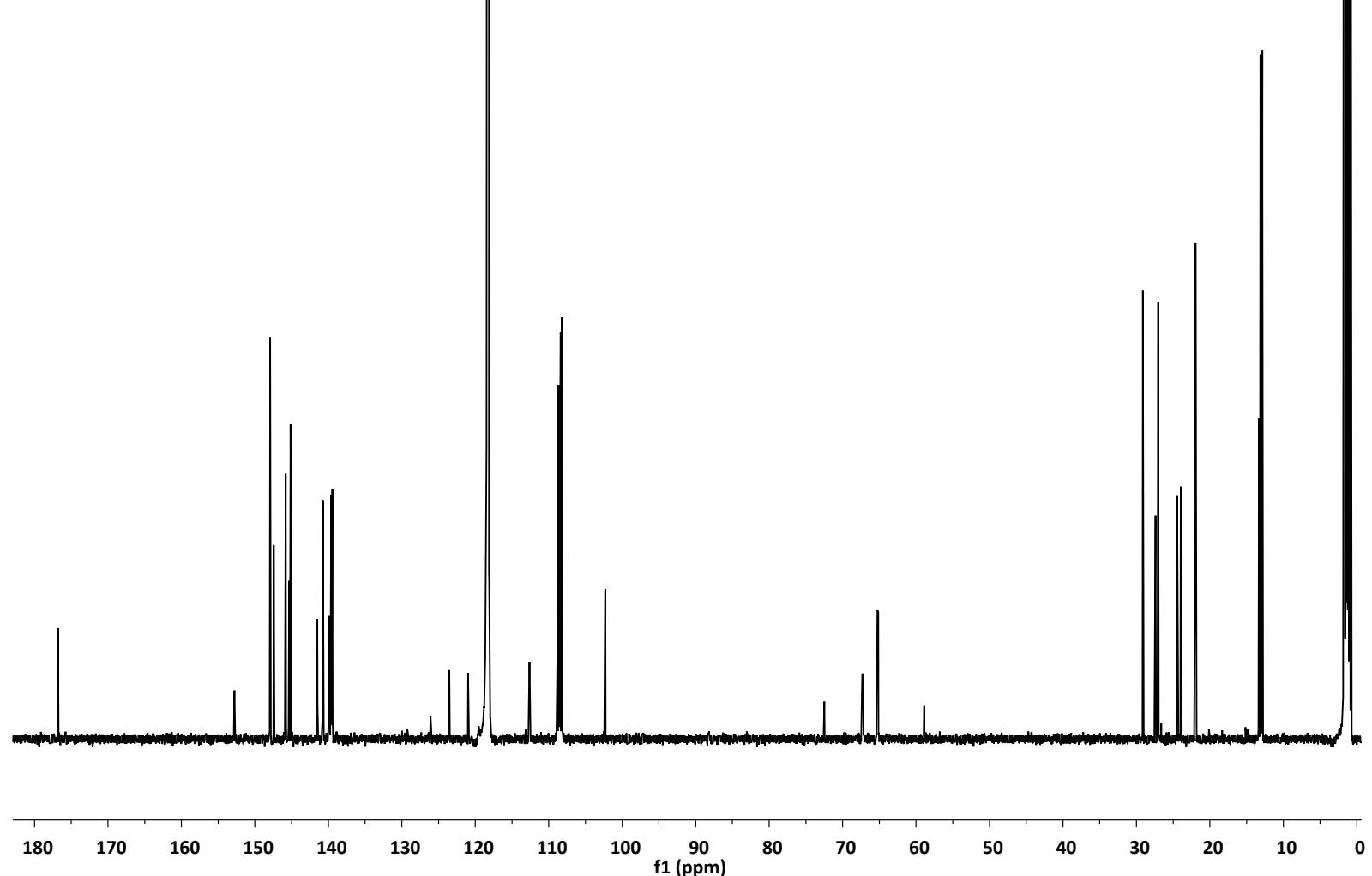
17, ${ }^{1} \mathrm{H}, \mathrm{CDCl}_{3}$
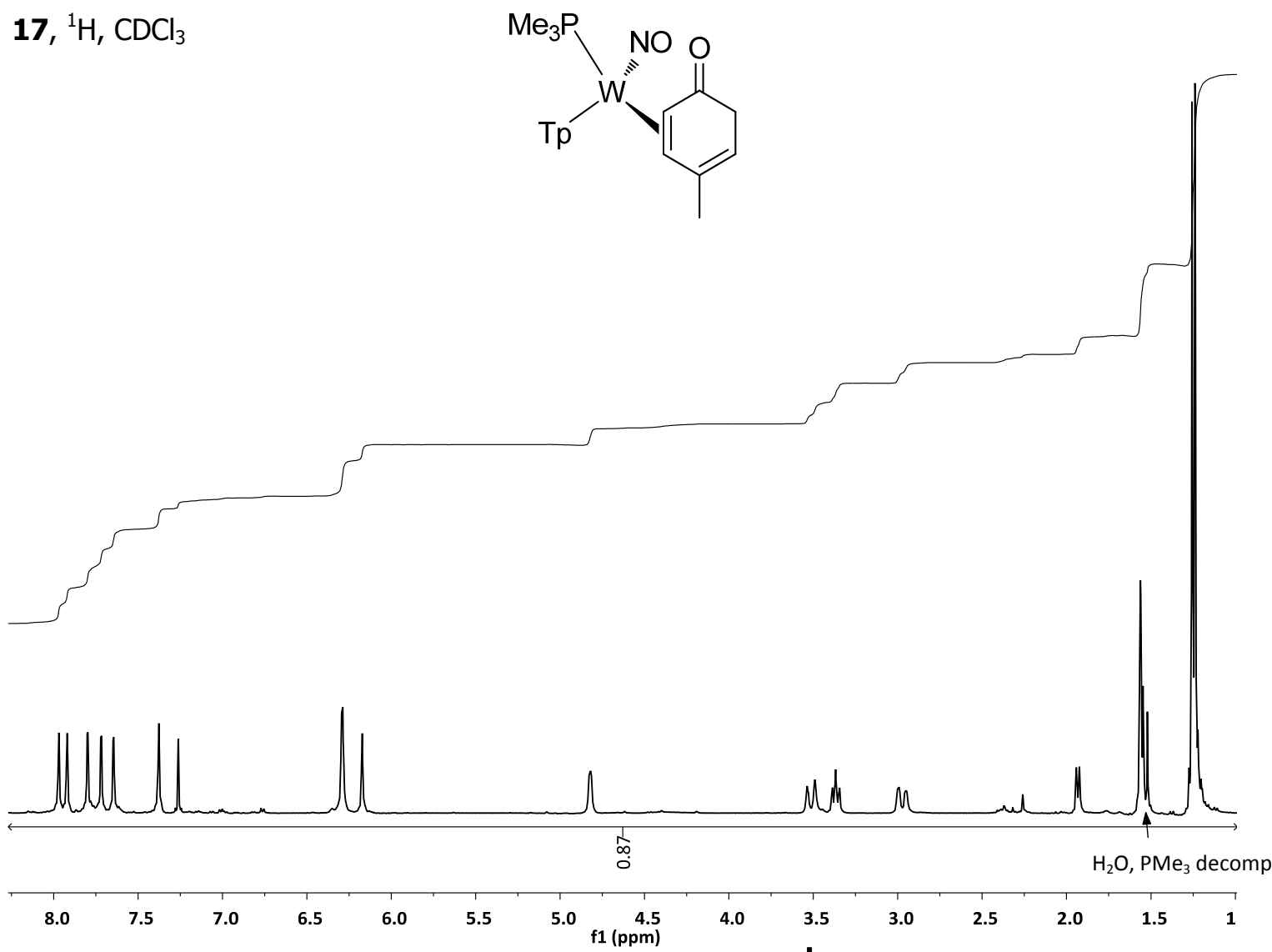

17, ${ }^{13} \mathrm{C}, \mathrm{CDCl}_{3}$

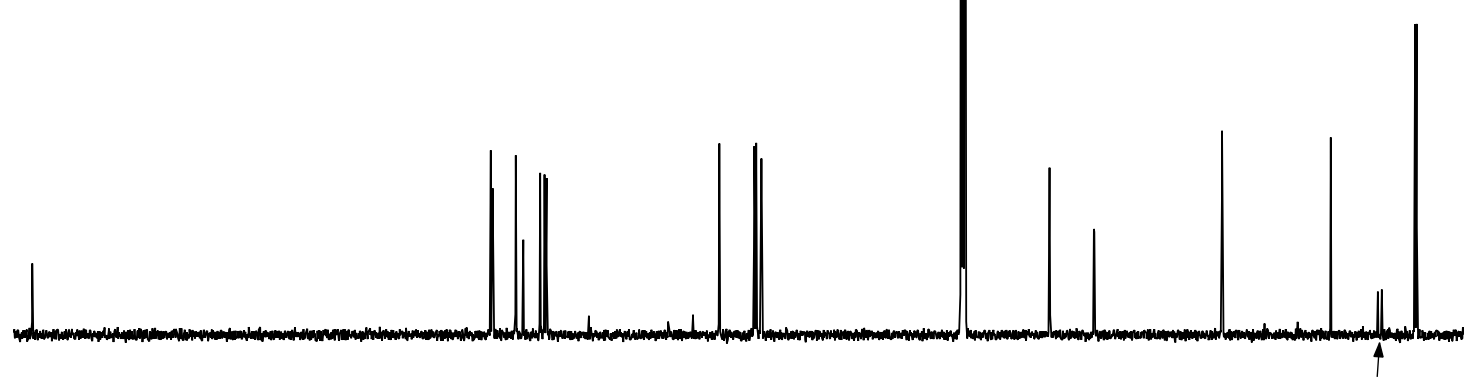

$\mathrm{PMe}_{3}$ decomp.

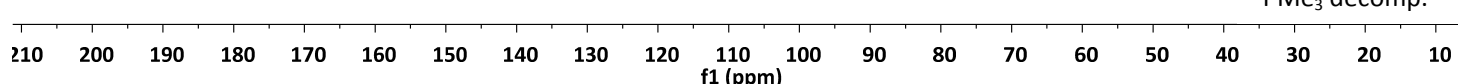




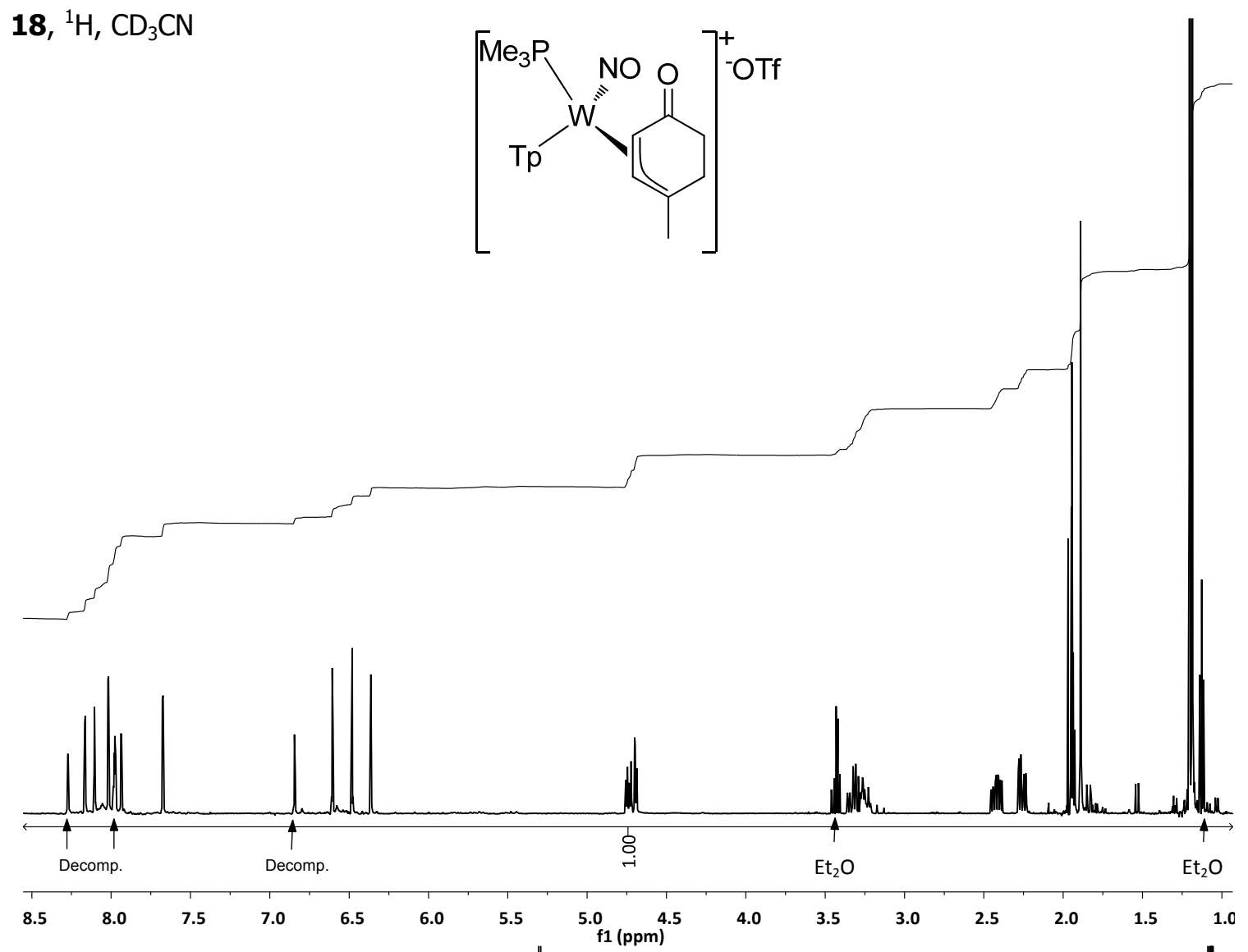

18, ${ }^{13} \mathrm{C}, \mathrm{CD}_{3} \mathrm{CN}$

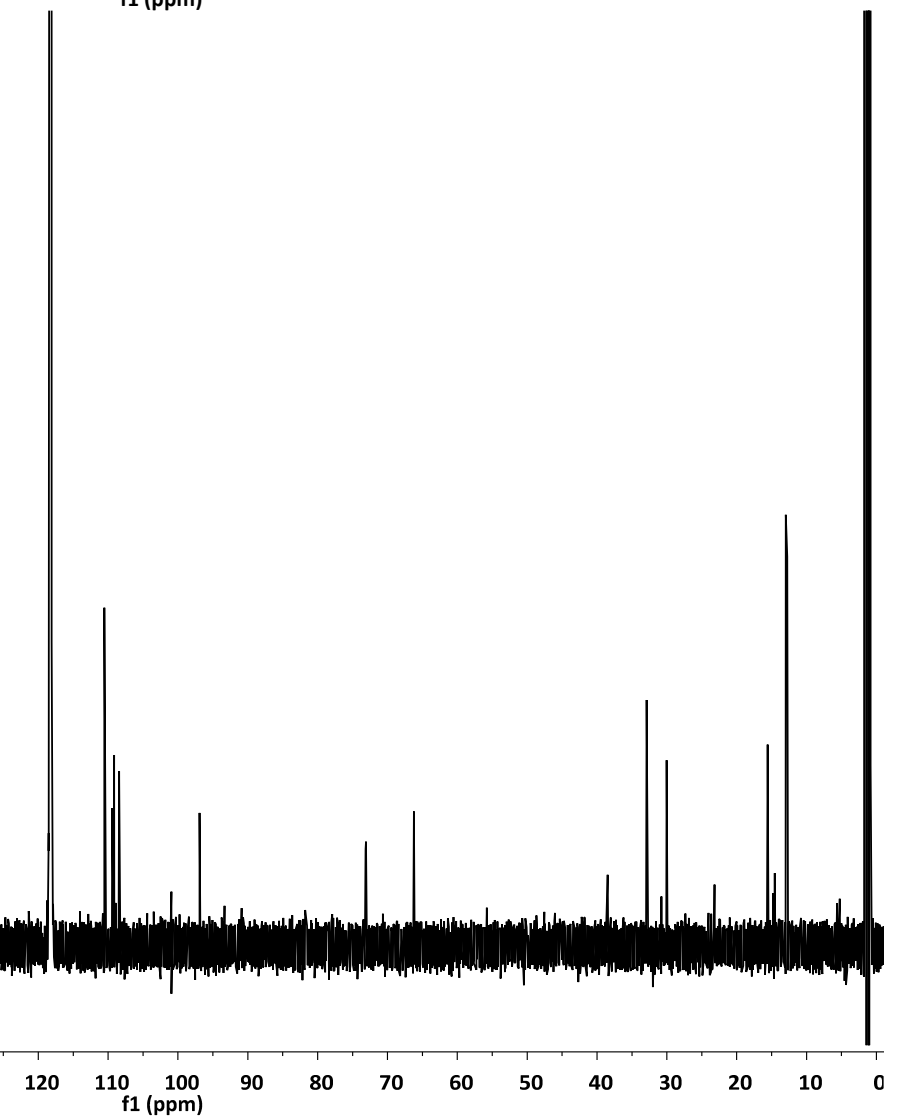




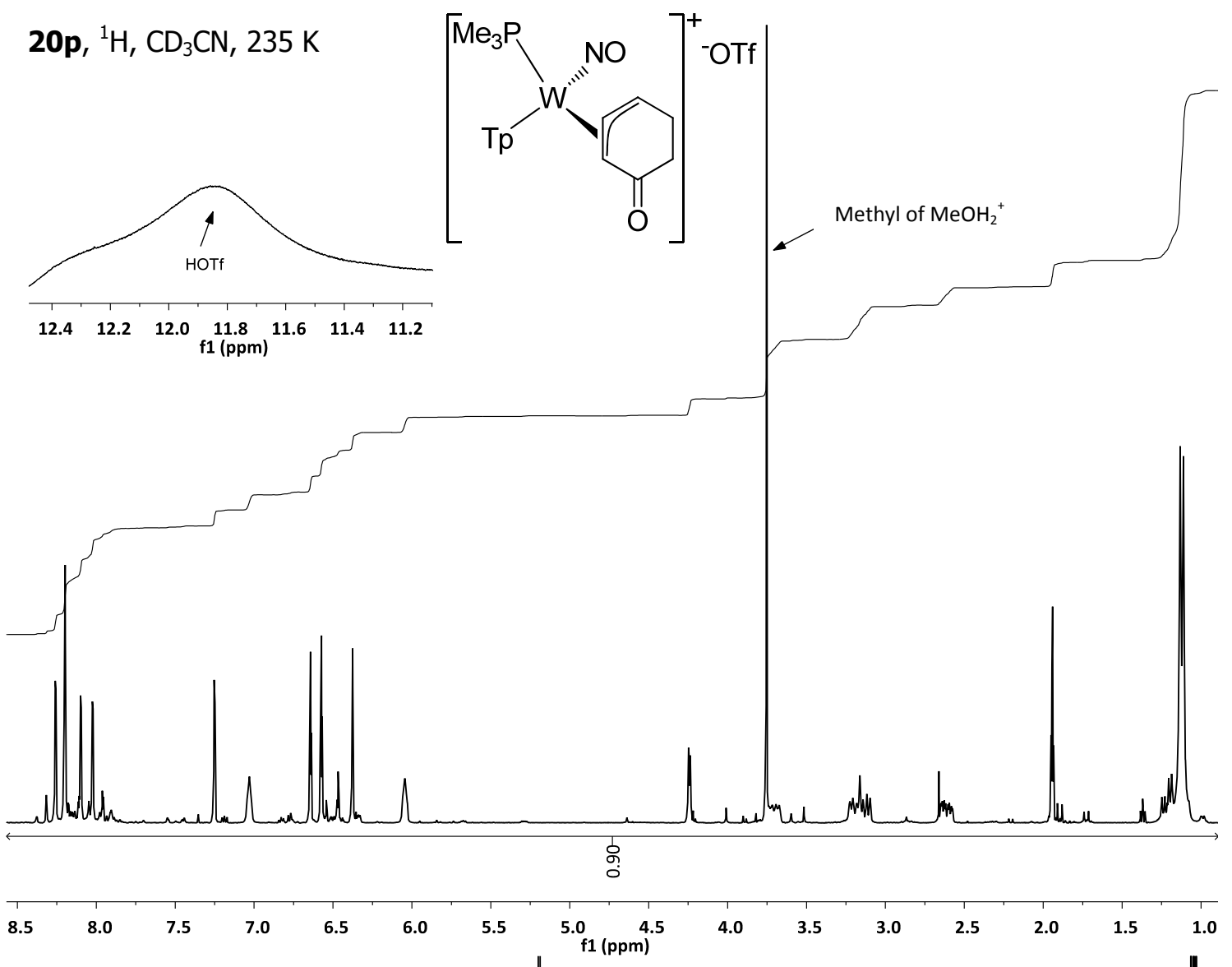

20p, ${ }^{13} \mathrm{C}, \mathrm{CD}_{3} \mathrm{CN}, 235 \mathrm{~K}$
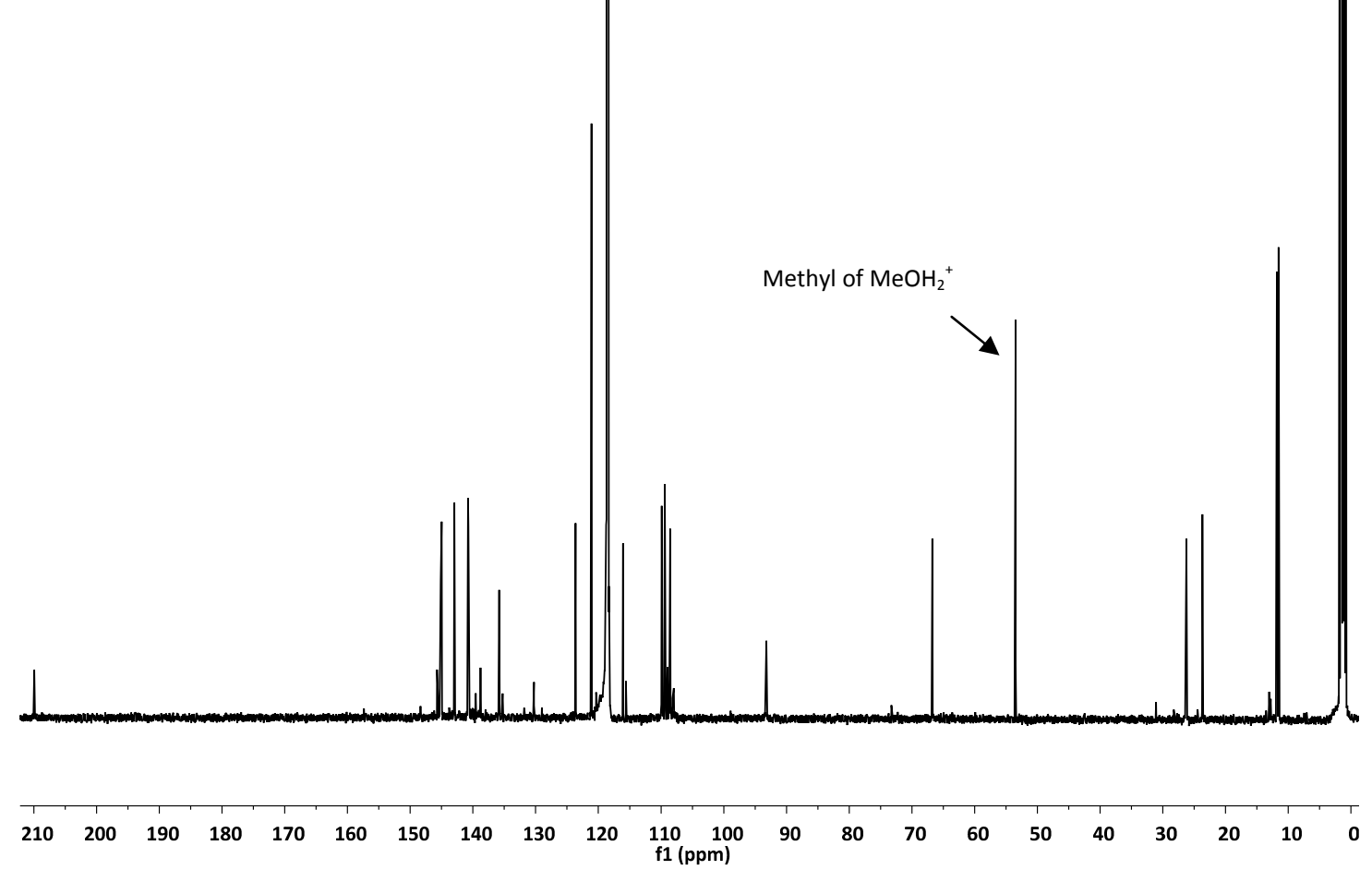
20p, 20d, ${ }^{1} \mathrm{H}, \mathrm{CD}_{3} \mathrm{CN}, 235 \mathrm{~K}$
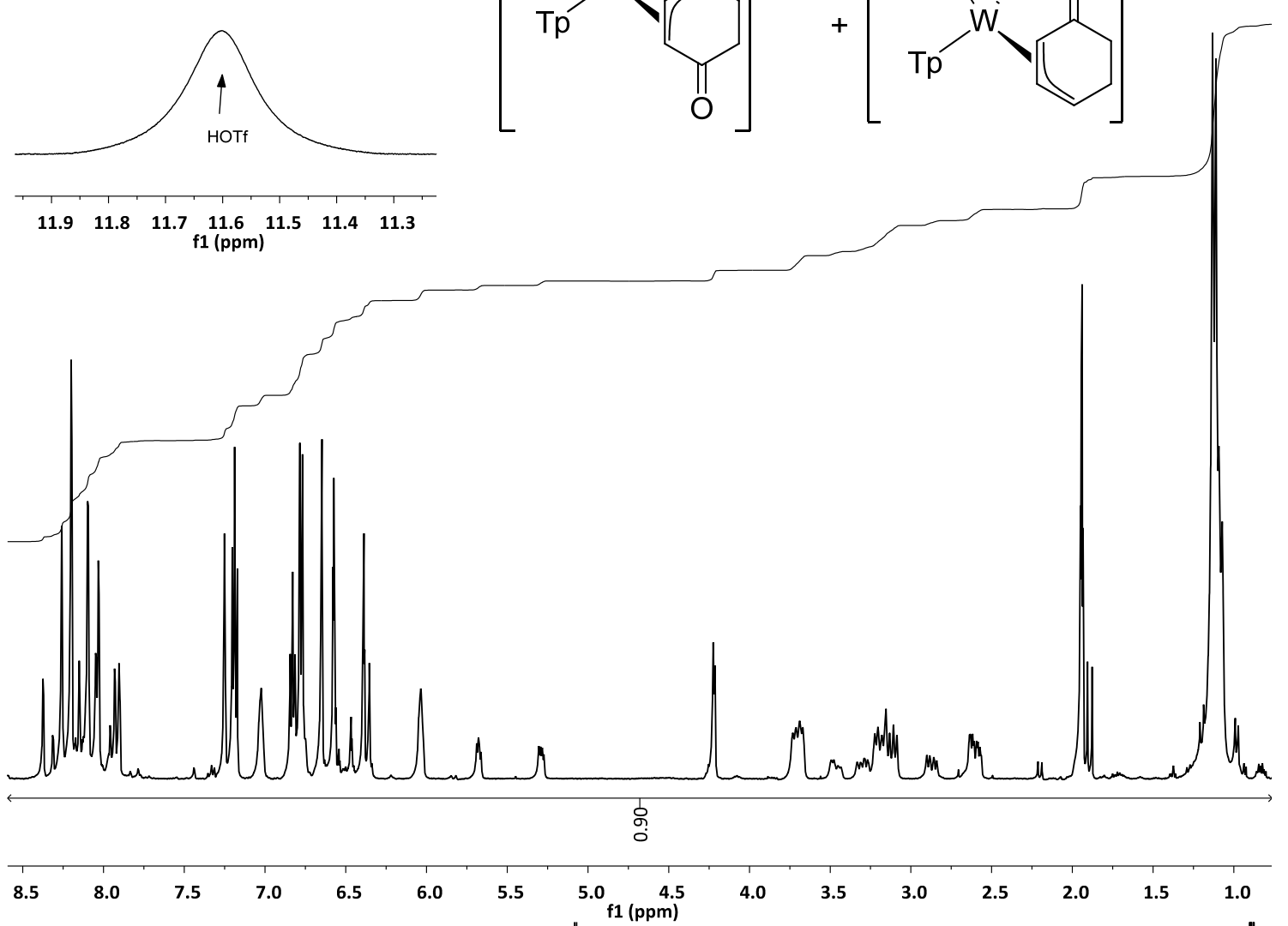

20p,20d, ${ }^{13} \mathrm{C}_{,} \mathrm{CD}_{3} \mathrm{CN}, 235 \mathrm{~K}$

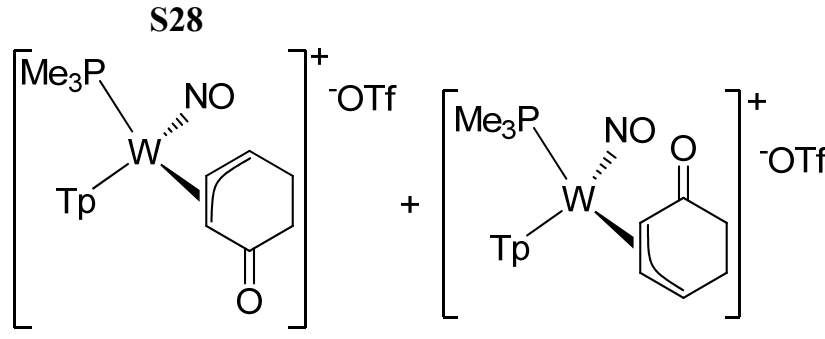


21p, ${ }^{1} \mathrm{H}, \mathrm{CD}_{3} \mathrm{CN}$
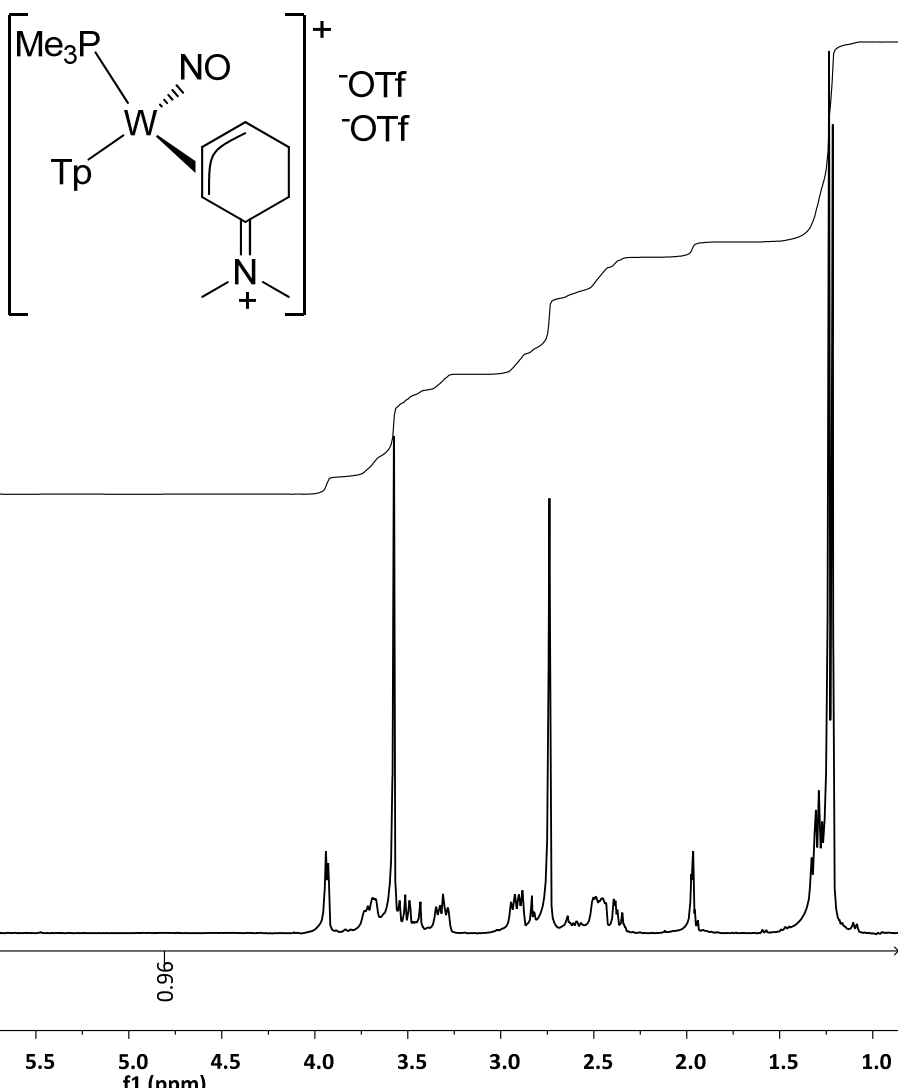

21p, ${ }^{13} \mathrm{C}, \mathrm{CD}_{3} \mathrm{CN}$

${ }_{\text {f1 }}^{5.0}(\mathrm{ppm})$

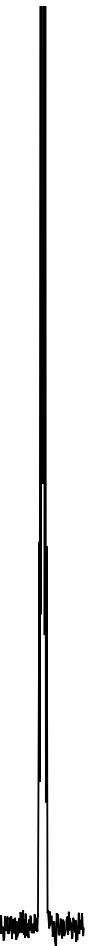

(n)
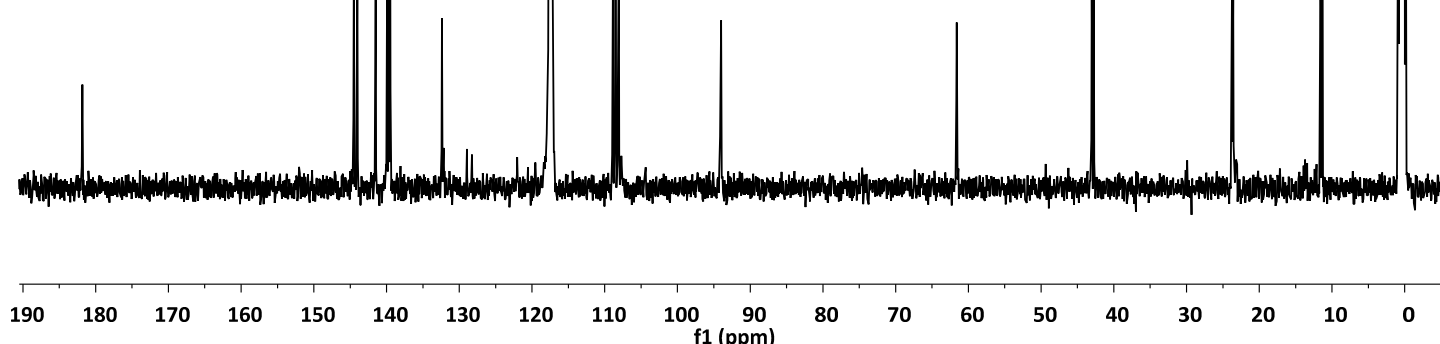
Exo-22, ${ }^{1} \mathrm{H}, \mathrm{CDCl}_{3}$
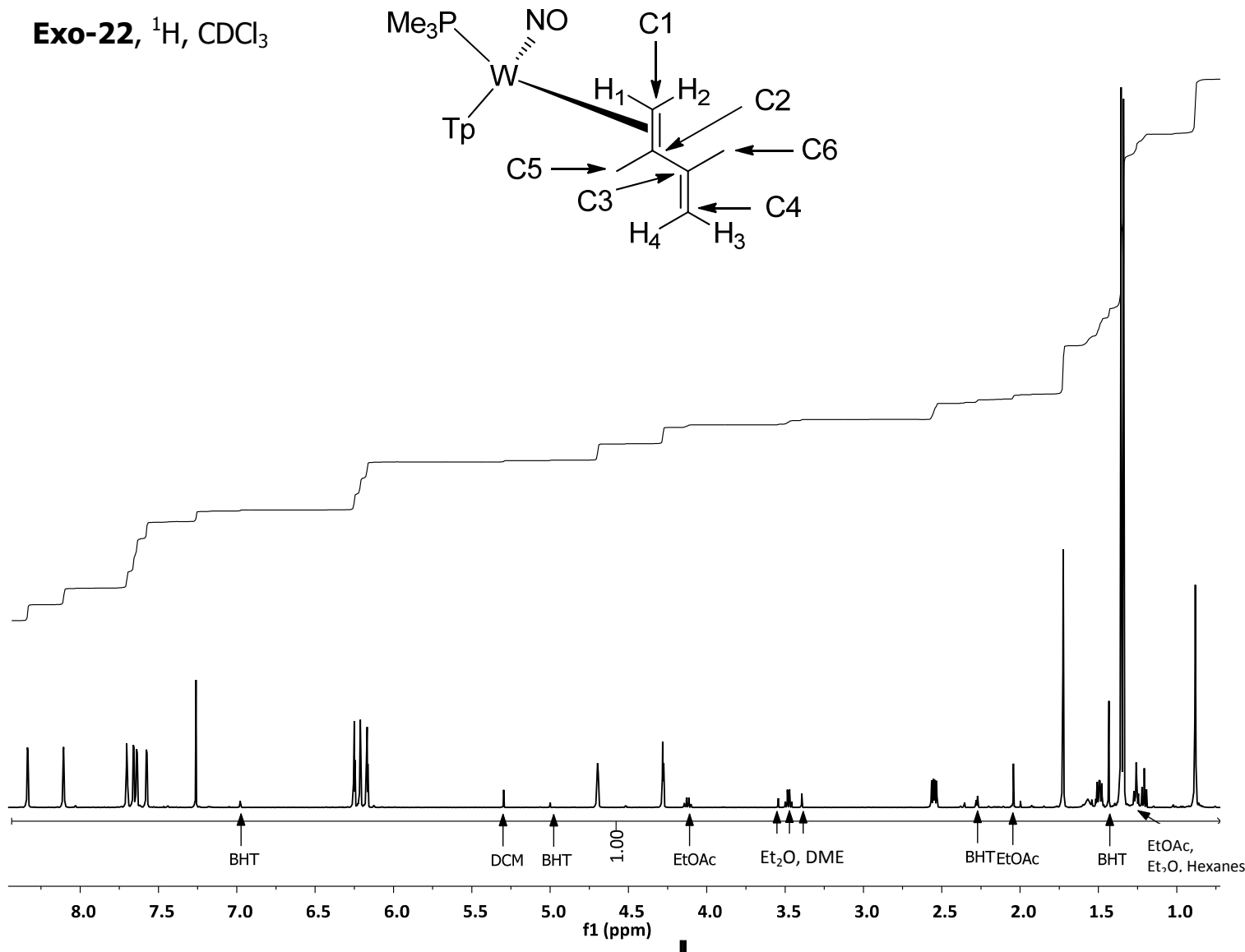

Exo-22, ${ }^{13} \mathrm{C}, \mathrm{CDCl}_{3}$

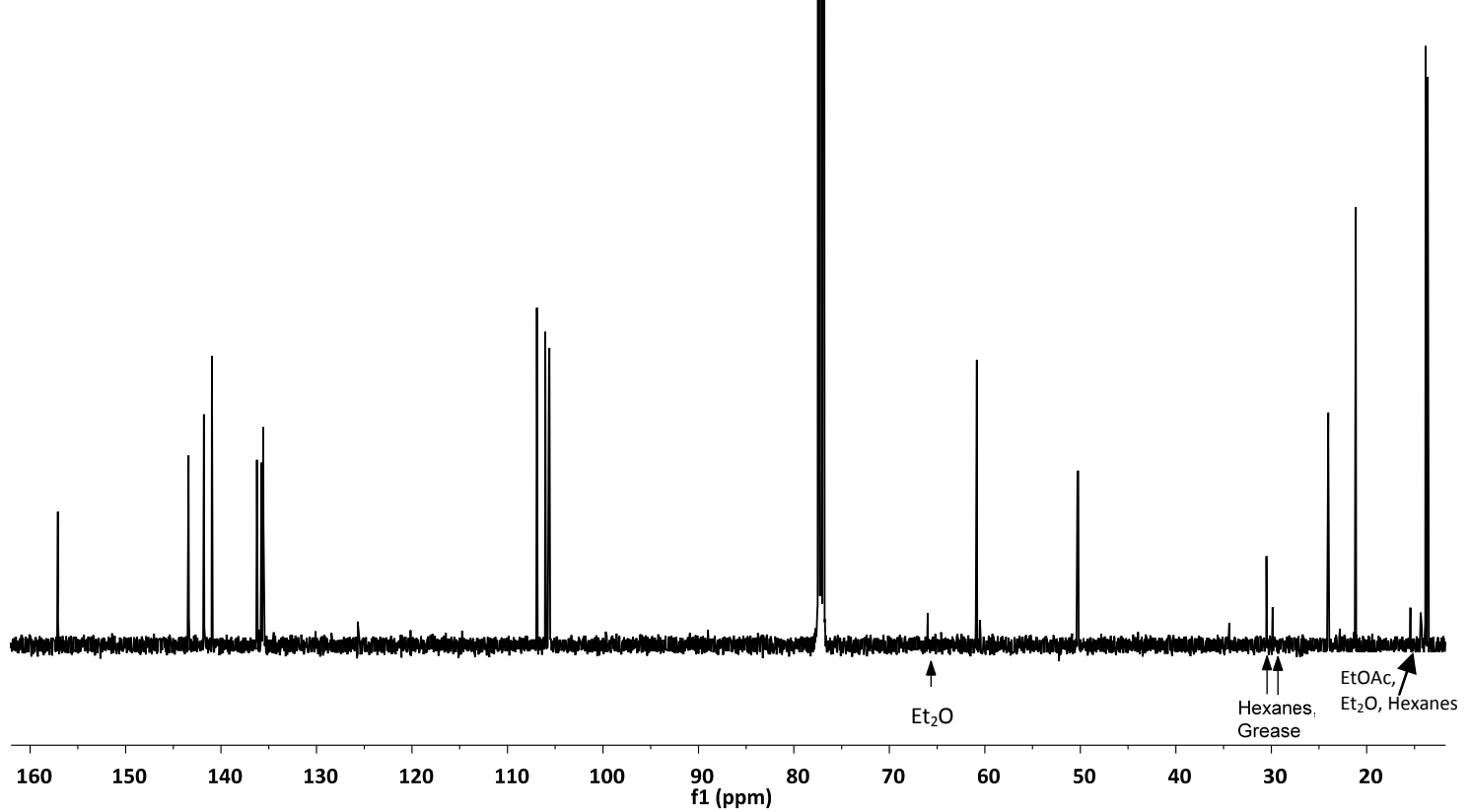


Exo-22, Endo-22, ${ }^{1} \mathrm{H}, \mathrm{CDCl}_{3}$

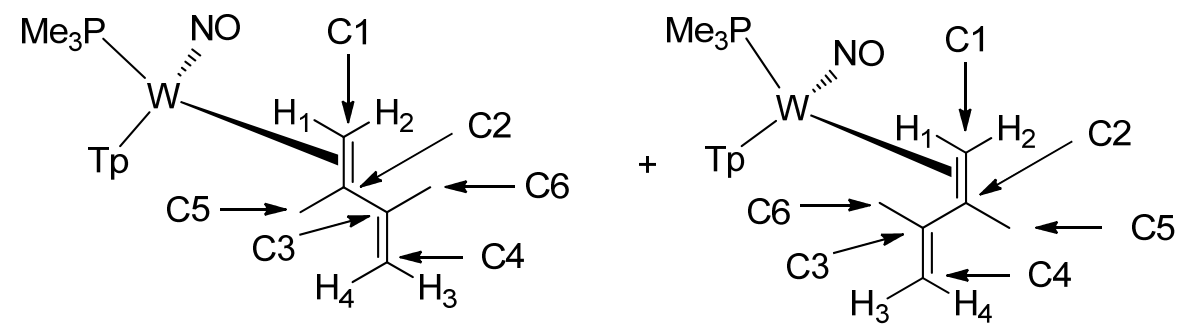

Exo
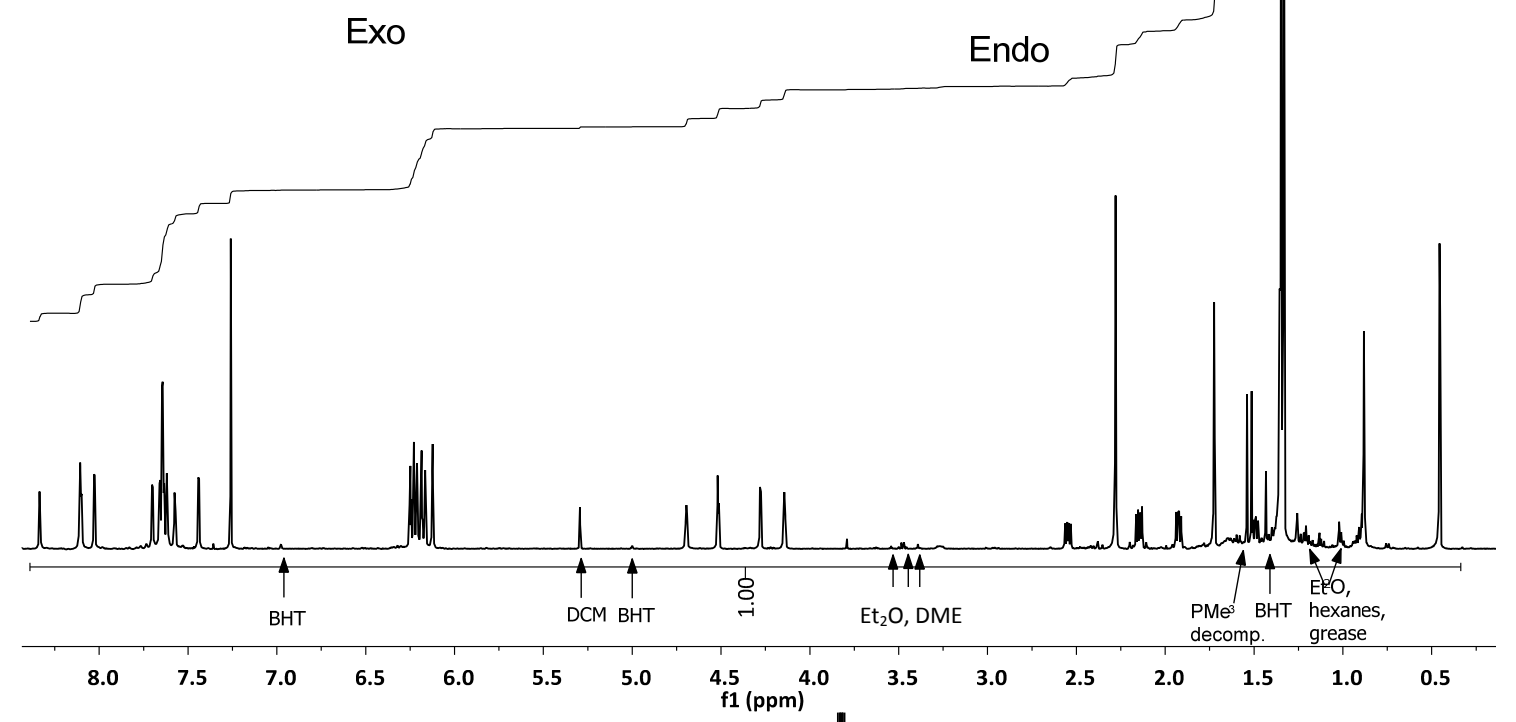

Exo-22, Endo-22, ${ }^{13} \mathrm{C}, \mathrm{CDCl}_{3}$

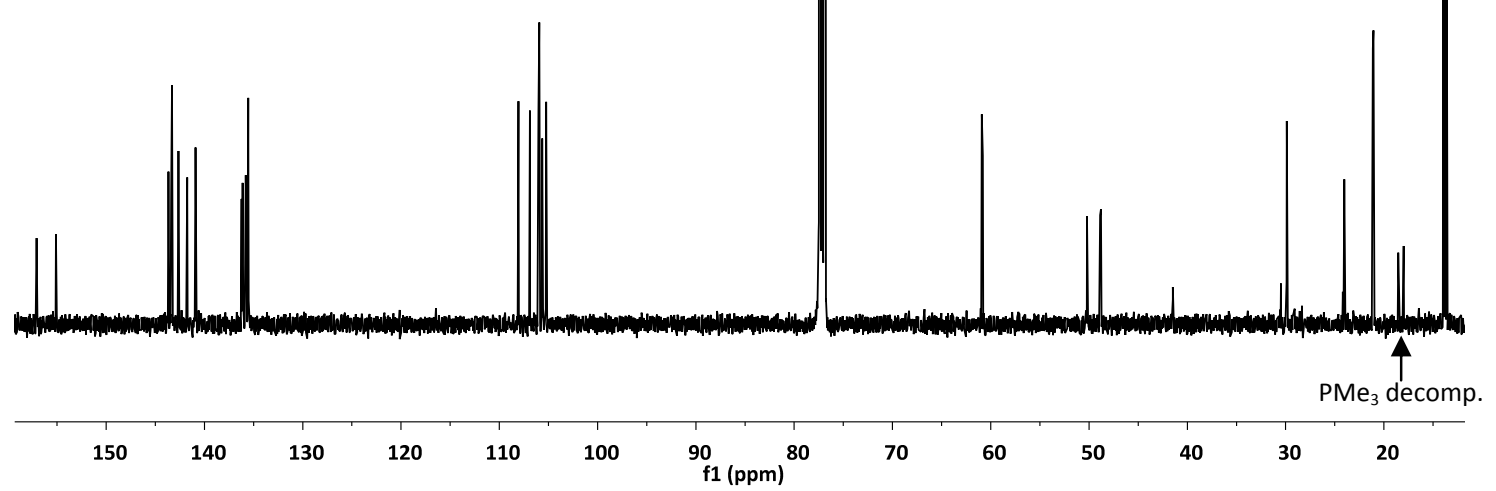


Exo-23, Endo-23, ${ }^{1} \mathrm{H}, \mathrm{CDCl}_{3}$

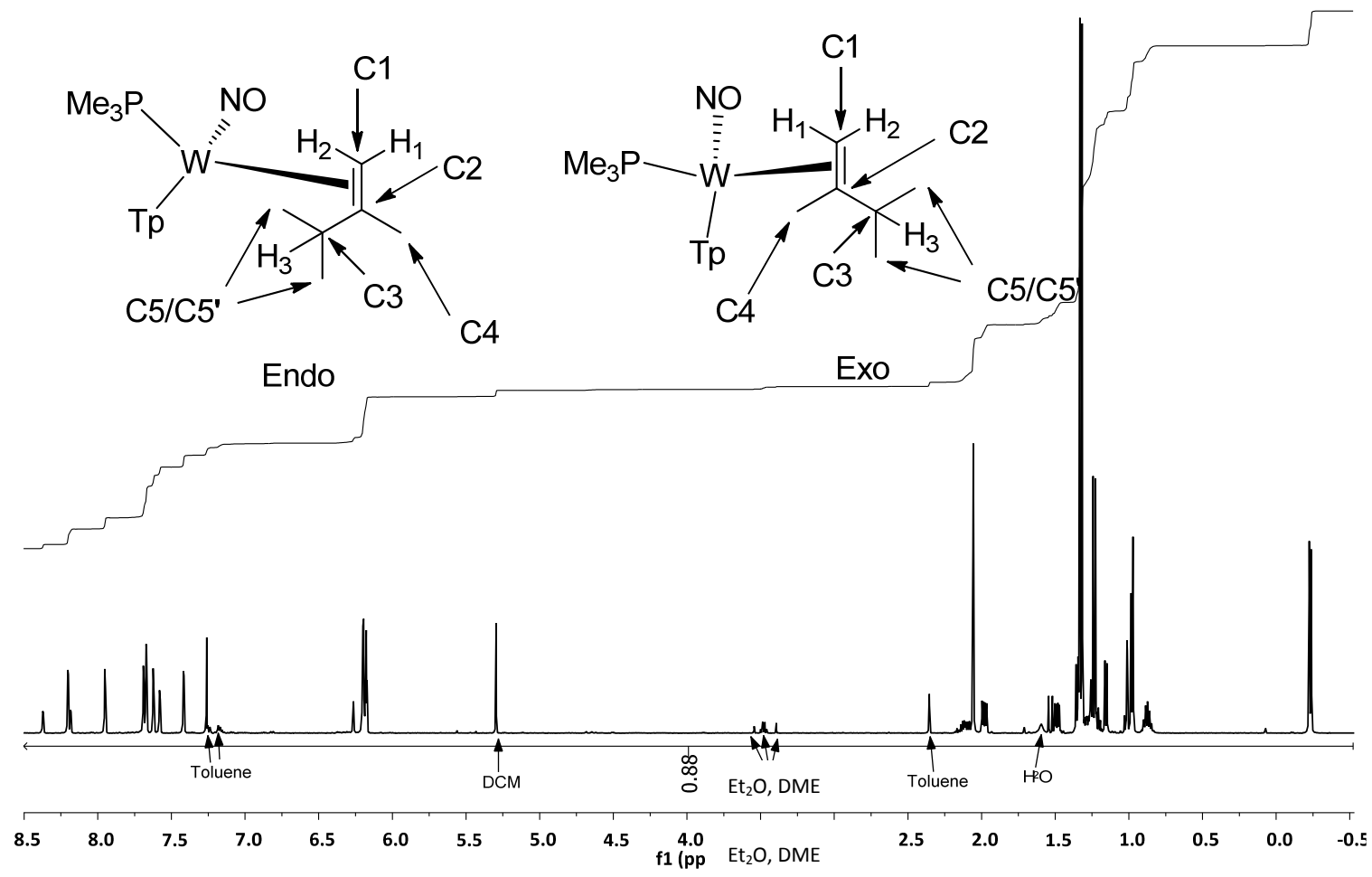

Exo-23, Endo-23, ${ }^{13} \mathrm{C}, \mathrm{CDCl}_{3}$

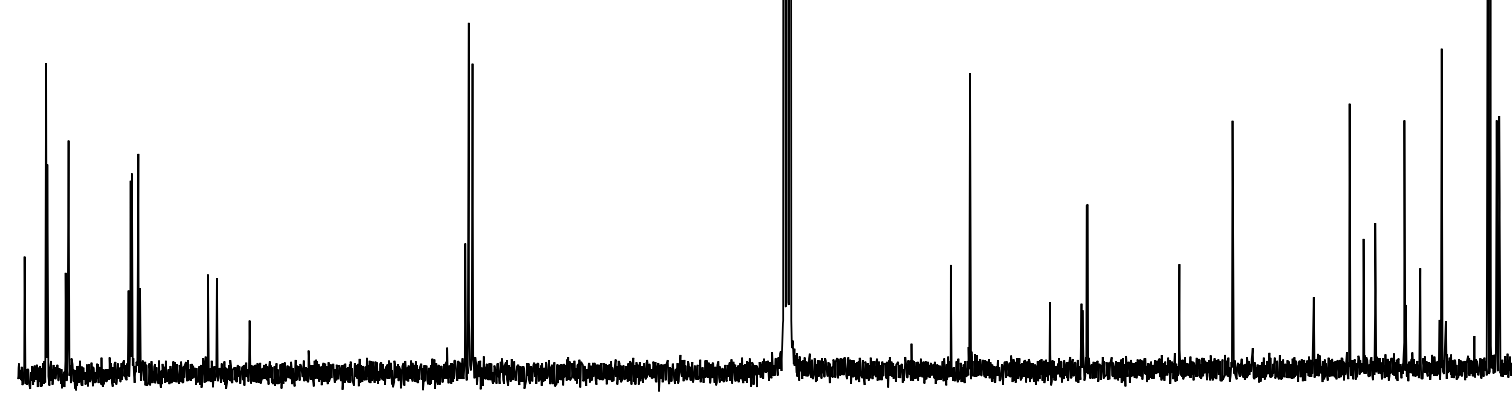

$\begin{array}{lllllllllllllllllllllllllllll}145 & 140 & 135 & 130 & 125 & 120 & 115 & 110 & 105 & 100 & 95 & 90 & 85 & \begin{array}{c}80 \\ f 1(\mathrm{ppm})\end{array} & 70 & 65 & 60 & 55 & 50 & 45 & 40 & 35 & 30 & 25 & 20 & 15\end{array}$ 
$\mathrm{Na} / \mathrm{Hg}$ Reduction of Exo-12 and Endo-12, ${ }^{1} \mathrm{H}, \mathrm{CDCl}_{3}$

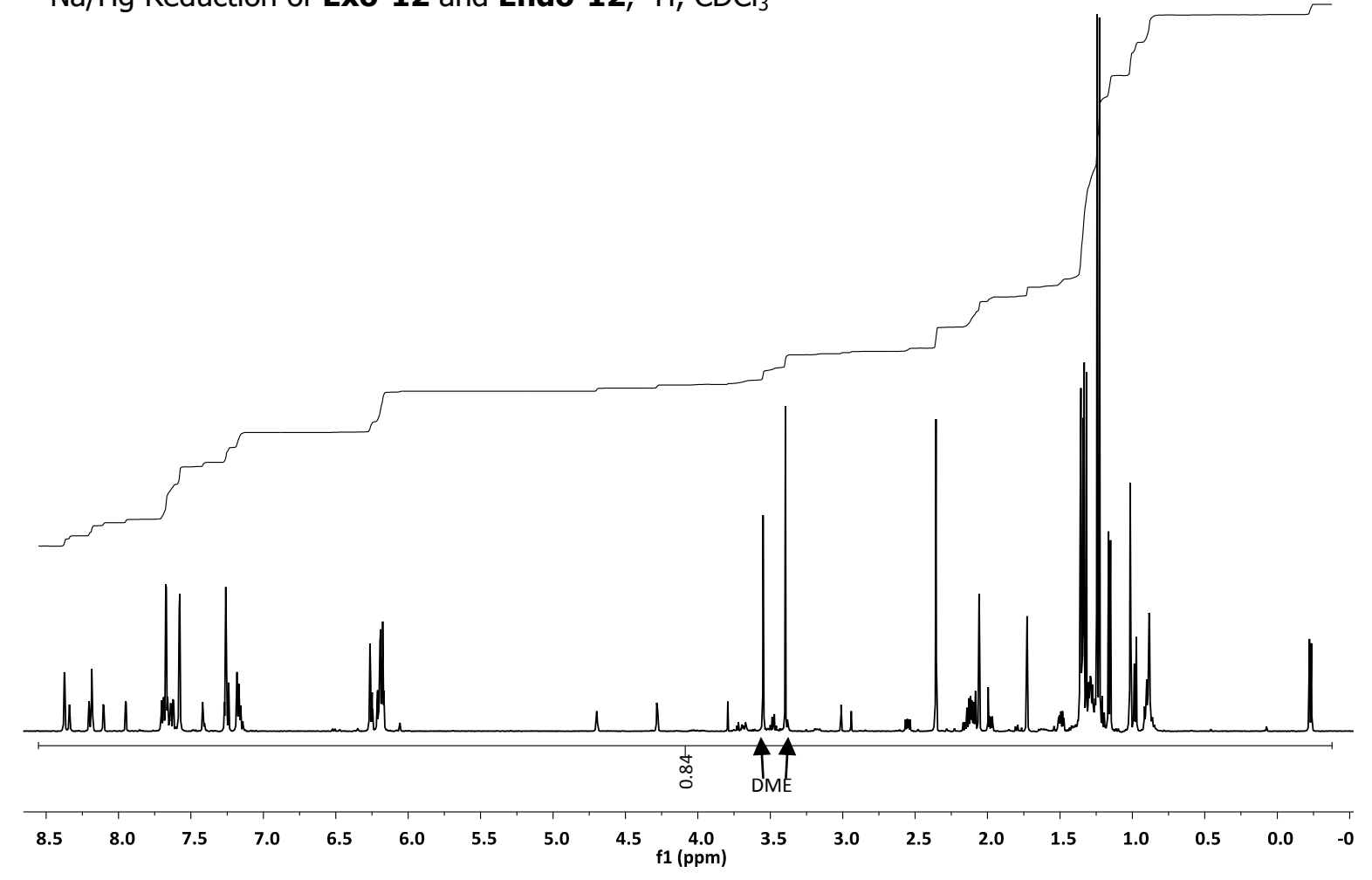


Table S1: Calculated Structural Energy and Number of Associated Imaginary Vibrational Frequencies for Compounds Numbered in Article Text.

\begin{tabular}{|c|c|c|c|}
\hline Compound & $\begin{array}{c}\text { Absolute Energy } \\
\text { (Hartree atomic units: au) }\end{array}$ & $\begin{array}{c}\text { Zero Point Corrected } \\
\text { Energy (au) }\end{array}$ & $\begin{array}{c}\text { Imaginary } \\
\text { Vibrational } \\
\text { Frequencies }\end{array}$ \\
\hline Exo 11 & -1478.465849 & -1478.064244 & 0 \\
\hline Endo 11 & -1478.465041 & -1478.06394 & 0 \\
\hline Exo 12 & -1596.408649 & -1595.923159 & 0 \\
\hline Endo 12 & -1596.406055 & -1595.920688 & 0 \\
\hline Exo 5 & -1763.887287 & -1763.394126 & 0 \\
\hline Exo 3d & -1595.200015 & -1594.733237 & 0 \\
\hline Exo $3 p$ & -1595.193581 & -1594.72727 & 0 \\
\hline Exo 3ts & -1595.190801 & -1594.723404 & 1 \\
\hline Endo 3 & -1595.183965 & -1594.716804 & 0 \\
\hline Exo 7d & -1763.477483 & -1762.998468 & 0 \\
\hline Exo 13 & -1708.124743 & -1707.663613 & 0 \\
\hline Exo 14 & -1727.971979 & -1727.455698 & 0 \\
\hline $11 \kappa$ & -1478.437402 & -1478.039955 & 0 \\
\hline $12 \mathrm{k}$ & -1596.387245 & -1596.571243 & 0 \\
\hline $12^{\cdot}$ & -1596.571243 & -1596.090644 & 0 \\
\hline $12^{-}$ & -1596.593895 & -1596.116703 & 0 \\
\hline $20 p$ & -1669.212687 & -1668.764512 & 0 \\
\hline $20 q$ & -1669.222634 & -1668.774821 & 0 \\
\hline $21 p$ & -1728.265948 & -1727.735814 & 0 \\
\hline $21 d$ & -1728.258679 & -1727.727965 & 0 \\
\hline
\end{tabular}


Table S2: Calculated Structural Energy and Number of Associated Imaginary Vibrational Frequencies for Theoretical Tungsten Complexes

\begin{tabular}{|c|c|c|c|}
\hline Theoretical Tungsten Complexes & $\begin{array}{c}\text { Absolute Energy } \\
\text { (Hartree atomic } \\
\text { units: au) }\end{array}$ & $\begin{array}{c}\text { Zero Point } \\
\text { Energy Corrected } \\
\text { Energy (au) }\end{array}$ & $\begin{array}{c}\text { Imaginary } \\
\text { Vibrational } \\
\text { Frequencies }\end{array}$ \\
\hline$\left[\left(\mathrm{NH}_{3}\right)_{5} \mathrm{~W}\left(\mathrm{C}_{3} \mathrm{H}_{5}\right)\right]^{+}$ & -467.7330205 & -467.468285 & 0 \\
\hline$\left[\left(\mathrm{NH}_{3}\right)_{4} \mathrm{~W}(\mathrm{NO})\left(\mathrm{C}_{3} \mathrm{H}_{5}\right)\right]^{2+}$ Terminus 1 & -540.8542368 & -540.614063 & 0 \\
\hline$\left[\left(\mathrm{NH}_{3}\right)_{4} \mathrm{~W}(\mathrm{NO})\left(\mathrm{C}_{3} \mathrm{H}_{5}\right)\right]^{2+}$ Terminus 2 & -540.8542377 & -540.614073 & 0 \\
\hline $\begin{array}{l}{\left[\left(\mathrm{NH}_{3}\right)_{4} \mathrm{~W}(\mathrm{NO})\left(\mathrm{C}_{3} \mathrm{H}_{5}\right)\right]^{2+}} \\
\text { Transition State }\end{array}$ & -540.8521158 & -540.612266 & 1 \\
\hline$\left[\left(\mathrm{NH}_{3}\right)_{4} \mathrm{~W}(\mathrm{NO})\left(\mathrm{C}_{6} \mathrm{H}_{9}\right)\right]^{2+}$ Terminus 1 & -657.5993325 & -657.294482 & 0 \\
\hline$\left[\left(\mathrm{NH}_{3}\right)_{4} \mathrm{~W}(\mathrm{NO})\left(\mathrm{C}_{6} \mathrm{H}_{9}\right)\right]^{2+}$ Terminus 2 & -657.5995332 & -657.294432 & 0 \\
\hline $\begin{array}{l}{\left[\left(\mathrm{NH}_{3}\right)_{4} \mathrm{~W}(\mathrm{NO})\left(\mathrm{C}_{6} \mathrm{H}_{9}\right)\right]^{2+}} \\
\text { Transition State }\end{array}$ & -657.5904836 & -657.28511 & 1 \\
\hline$\left\{\mathrm{TpW}(\mathrm{NO})\left(\mathrm{PMe}_{3}\right)\right\}$ & -1361.298575 & -1360.973183 & 0 \\
\hline$\left[\left(\mathrm{NH}_{3}\right)_{3} \mathrm{~W}\left(\mathrm{PMe}_{3}\right)(\mathrm{NO})\left(\mathrm{C}_{6} \mathrm{H}_{9}\right)\right]^{2+}$ Distal & -1062.156214 & -1061.774368 & 0 \\
\hline $\begin{array}{c}{\left[\left(\mathrm{NH}_{3}\right)_{3} \mathrm{~W}\left(\mathrm{PMe}_{3}\right)(\mathrm{NO})\left(\mathrm{C}_{6} \mathrm{H}_{9}\right)\right]^{2+}} \\
\text { Proximal }\end{array}$ & -1062.155776 & -1061.774086 & 0 \\
\hline $\begin{array}{c}{\left[\left(\mathrm{NH}_{3}\right)_{3} \mathrm{~W}\left(\mathrm{PMe}_{3}\right)(\mathrm{NO})\left(\mathrm{C}_{6} \mathrm{H}_{9}\right)\right]^{2+}} \\
\text { Transition State }\end{array}$ & -1062.149113 & -1061.766637 & 1 \\
\hline$\left[\mathrm{TpW}(\mathrm{NO})\left(\mathrm{NH}_{3}\right)\left(\mathrm{C}_{6} \mathrm{H}_{9}\right)\right]^{+}$Distal & -1190.658467 & -1190.267798 & 0 \\
\hline$\left[\mathrm{TpW}(\mathrm{NO})\left(\mathrm{NH}_{3}\right)\left(\mathrm{C}_{6} \mathrm{H}_{9}\right)\right]^{+}$Proximal & -1190.651300 & -1190.261245 & 0 \\
\hline
\end{tabular}


Cartesian coordinates and Bond Connectivity for Calculated Structures:

Protein database files (PDB) were created for each of the optimized structures using GaussView 5.0.8. The list of atoms, their Cartesian coordinates, and their connectivity are provided in the text of PDB files and presented here. Images from the optimized Gaussian structures are provided at the end of each of the connectivity tables.

PDB Data: Table of Contents

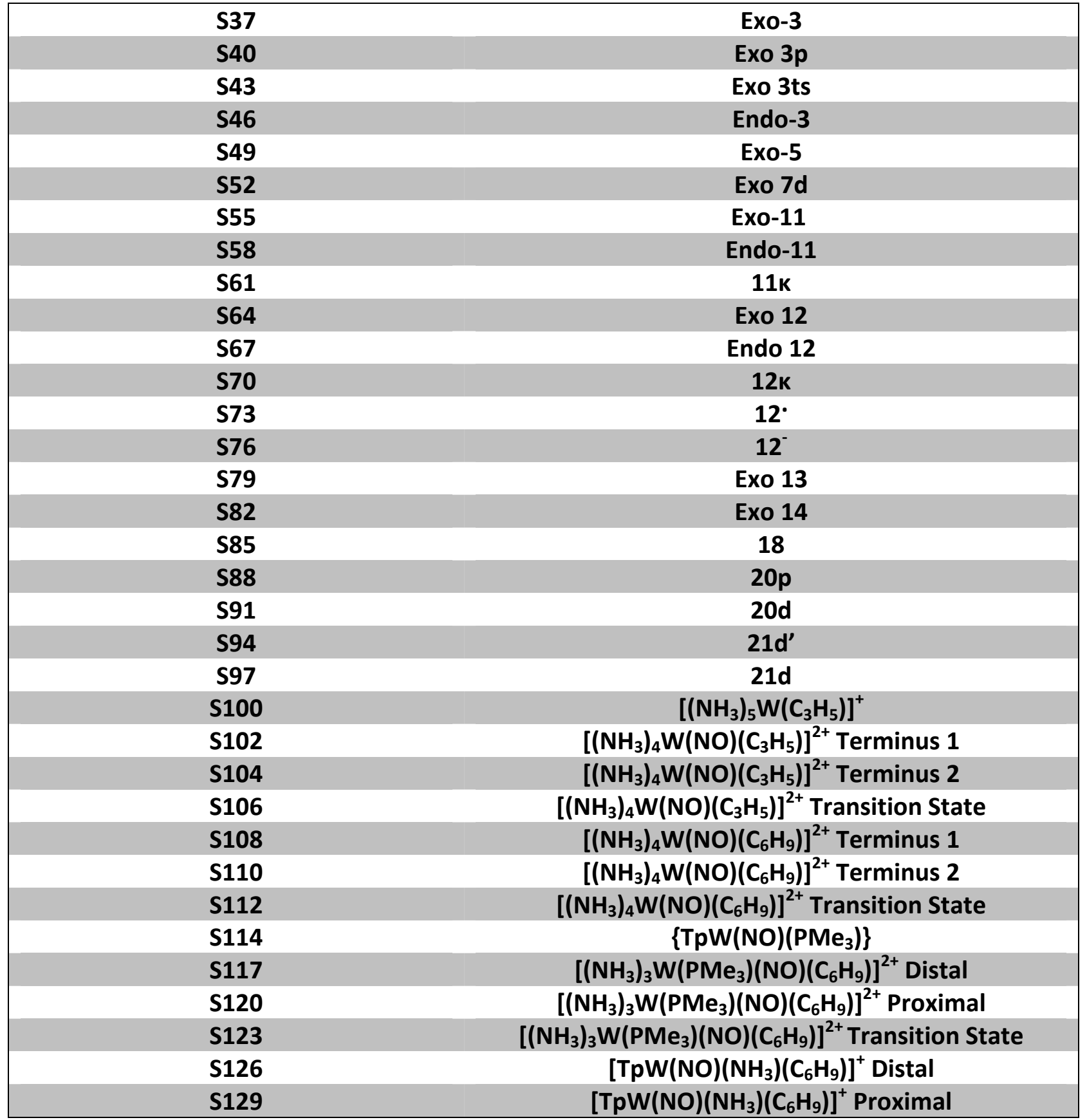




\begin{tabular}{|c|c|c|c|c|c|c|c|}
\hline TITLE & Exo-3 & & & & & & \\
\hline REMARK & 1 & File & eat & y Gauss & View 5. & 0.8 & \\
\hline HETATM & 1 & $B$ & 0 & 2.944 & -0.005 & 0.48 & \\
\hline HETATM & 2 & $\mathrm{~N}$ & 0 & 2.535 & -0.784 & -0.8 & \\
\hline HETATM & 3 & $\mathrm{~N}$ & 0 & 2.532 & 1.466 & 0.226 & \\
\hline HETATM & 4 & $\mathrm{~N}$ & 0 & 2.177 & -0.557 & 1.69 & \\
\hline HETATM & 5 & $\mathrm{H}$ & 0 & 4.124 & -0.088 & 0.66 & \\
\hline HETATM & 6 & $N$ & 0 & 1.258 & -0.684 & -1.269 & \\
\hline HETATM & 7 & C & 0 & 1.272 & -1.112 & -2.546 & \\
\hline HETATM & 8 & C & 0 & 2.56 & -1.516 & -2.905 & \\
\hline HETATM & 9 & C & 0 & 3.329 & -1.279 & -1.772 & \\
\hline HETATM & 10 & C & 0 & 2.656 & -1.068 & 2.842 & \\
\hline HETATM & 11 & C & 0 & 1.584 & -1.399 & 3.659 & \\
\hline HETATM & 12 & C & 0 & 0.456 & -1.047 & 2.915 & \\
\hline HETATM & 13 & $\mathrm{~N}$ & 0 & 0.815 & -0.537 & 1.72 & \\
\hline HETATM & 14 & C & 0 & 3.341 & 2.526 & 0.016 & \\
\hline HETATM & 15 & C & 0 & 2.585 & 3.575 & -0.488 & \\
\hline HETATM & 16 & C & 0 & 1.288 & 3.063 & -0.571 & \\
\hline HETATM & 17 & $\mathrm{~N}$ & 0 & 1.252 & 1.793 & -0.128 & \\
\hline HETATM & 18 & $\mathrm{H}$ & 0 & 0.369 & -1.089 & -3.137 & \\
\hline HETATM & 19 & $\mathrm{H}$ & 0 & 2.889 & -1.908 & -3.855 & \\
\hline HETATM & 20 & $\mathrm{H}$ & 0 & 4.387 & -1.41 & -1.596 & \\
\hline HETATM & 21 & $\mathrm{H}$ & 0 & 3.722 & -1.155 & 2.999 & \\
\hline HETATM & 22 & $\mathrm{H}$ & 0 & 1.617 & -1.826 & 4.651 & \\
\hline HETATM & 23 & $\mathrm{H}$ & 0 & -0.586 & -1.127 & 3.189 & \\
\hline HETATM & 24 & $\mathrm{H}$ & 0 & 4.397 & 2.456 & 0.232 & \\
\hline HETATM & 25 & $\mathrm{H}$ & 0 & 2.923 & 4.563 & -0.76 & \\
\hline HETATM & 26 & $\mathrm{H}$ & 0 & 0.391 & 3.541 & -0.936 & \\
\hline HETATM & 27 & W & 0 & -0.348 & 0.236 & -0.078 & \\
\hline HETATM & 28 & $P$ & 0 & -1.197 & -2.227 & -0.291 & \\
\hline HETATM & 29 & C & 0 & -2.363 & -2.94 & 0.952 & \\
\hline HETATM & 30 & $\mathrm{H}$ & 0 & -3.313 & -2.401 & 0.957 & \\
\hline HETATM & 31 & $\mathrm{H}$ & 0 & -1.925 & -2.902 & 1.954 & \\
\hline HETATM & 32 & $\mathrm{H}$ & 0 & -2.556 & -3.988 & 0.701 & \\
\hline HETATM & 33 & C & 0 & -2.077 & -2.563 & -1.878 & \\
\hline HETATM & 34 & $\mathrm{H}$ & 0 & -1.414 & -2.39 & -2.729 & \\
\hline HETATM & 35 & $\mathrm{H}$ & 0 & -2.938 & -1.896 & -1.978 & \\
\hline HETATM & 36 & $\mathrm{H}$ & 0 & -2.422 & -3.601 & -1.908 & \\
\hline HETATM & 37 & $C$ & 0 & 0.155 & -3.484 & -0.249 & \\
\hline HETATM & 38 & $\mathrm{H}$ & 0 & 0.859 & -3.331 & -1.069 & $\theta$ \\
\hline HETATM & 39 & $\mathrm{H}$ & 0 & -0.273 & -4.489 & -0.329 & \\
\hline HETATM & 40 & $\mathrm{H}$ & 0 & 0.697 & -3.404 & 0.698 & 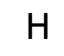 \\
\hline HETATM & 41 & $\mathrm{~N}$ & 0 & -1.207 & 0.684 & -1.578 & \\
\hline HETATM & 42 & 0 & 0 & -1.789 & 0.936 & -2.591 & \\
\hline
\end{tabular}




\begin{tabular}{|c|c|c|c|c|c|c|}
\hline HETATM & 43 & C & 0 & -1.613 & 2.488 & 0.727 \\
\hline HETATM & 44 & C & 0 & -1.519 & 1.397 & 1.578 \\
\hline HETATM & 45 & C & 0 & -2.851 & 2.743 & -0.095 \\
\hline HETATM & 46 & $\mathrm{H}$ & 0 & -2.676 & 2.619 & -1.171 \\
\hline HETATM & 47 & $\mathrm{H}$ & 0 & -0.818 & 1.421 & 2.407 \\
\hline HETATM & 48 & C & 0 & -4.006 & 1.844 & 0.383 \\
\hline HETATM & 49 & $\mathrm{H}$ & 0 & -4.86 & 1.939 & -0.295 \\
\hline HETATM & 50 & C & 0 & -3.592 & 0.366 & 0.505 \\
\hline HETATM & 51 & C & 0 & -2.248 & 0.205 & 1.244 \\
\hline HETATM & 52 & $\mathrm{H}$ & 0 & -2.247 & -0.556 & 2.018 \\
\hline HETATM & 53 & $\mathrm{H}$ & 0 & -4.334 & 2.2 & 1.367 \\
\hline HETATM & 54 & $\mathrm{H}$ & 0 & -3.116 & 3.8 & 0.037 \\
\hline HETATM & 55 & $\mathrm{H}$ & 0 & -0.916 & 3.307 & 0.87 \\
\hline HETATM & 56 & $\mathrm{H}$ & 0 & -4.369 & -0.172 & 1.062 \\
\hline HETATM & 57 & $\mathrm{H}$ & 0 & -3.562 & -0.083 & -0.493 \\
\hline \multicolumn{7}{|l|}{ END } \\
\hline CONECT & 1 & 2 & 3 & 4 & 5 & \\
\hline CONECT & 2 & 1 & 6 & 9 & & \\
\hline CONECT & 3 & 1 & 14 & 17 & & \\
\hline CONECT & 4 & 1 & 10 & 13 & & \\
\hline CONECT & 5 & 1 & & & & \\
\hline CONECT & 6 & 2 & 7 & & & \\
\hline CONECT & 7 & 6 & 8 & 18 & & \\
\hline CONECT & 8 & 7 & 9 & 19 & & \\
\hline CONECT & 9 & 8 & 2 & 20 & & \\
\hline CONECT & 10 & 4 & 11 & 21 & & \\
\hline CONECT & 11 & 10 & 12 & 22 & & \\
\hline CONECT & 12 & 11 & 13 & 23 & & \\
\hline CONECT & 13 & 4 & 12 & & & \\
\hline CONECT & 14 & 3 & 15 & 24 & & \\
\hline CONECT & 15 & 14 & 16 & 25 & & \\
\hline CONECT & 16 & 15 & 17 & 26 & & \\
\hline CONECT & 17 & 3 & 16 & & & \\
\hline CONECT & 18 & 7 & & & & \\
\hline CONECT & 19 & 8 & & & & \\
\hline CONECT & 20 & 9 & & & & \\
\hline CONECT & 21 & 10 & & & & \\
\hline CONECT & 22 & 11 & & & & \\
\hline CONECT & 23 & 12 & & & & \\
\hline CONECT & 24 & 14 & & & & \\
\hline CONECT & 25 & 15 & & & & \\
\hline CONECT & 26 & 16 & & & & \\
\hline CONECT & 27 & 41 & & & & \\
\hline CONECT & 28 & 29 & 33 & 37 & & \\
\hline
\end{tabular}




$\begin{array}{llllll}\text { CONECT } & 29 & 28 & 30 & 31 & 32 \\ \text { CONECT } & 30 & 29 & & & \\ \text { CONECT } & 31 & 29 & & & \\ \text { CONECT } & 32 & 29 & & & \\ \text { CONECT } & 33 & 28 & 34 & 35 & 36 \\ \text { CONECT } & 34 & 33 & & & \\ \text { CONECT } & 35 & 33 & & & \\ \text { CONECT } & 36 & 33 & & & \\ \text { CONECT } & 37 & 28 & 38 & 39 & 40 \\ \text { CONECT } & 38 & 37 & & & \\ \text { CONECT } & 39 & 37 & & & \\ \text { CONECT } & 40 & 37 & & & \\ \text { CONECT } & 41 & 27 & 42 & & \\ \text { CONECT } & 42 & 41 & & & \\ \text { CONECT } & 43 & 44 & 45 & 55 & \\ \text { CONECT } & 44 & 43 & 47 & 51 & \\ \text { CONECT } & 45 & 43 & 46 & 48 & 54 \\ \text { CONECT } & 46 & 45 & & & \\ \text { CONECT } & 47 & 44 & & & \\ \text { CONECT } & 48 & 45 & 49 & 50 & 53 \\ \text { CONECT } & 49 & 48 & & & \\ \text { CONECT } & 50 & 48 & 51 & 56 & 57 \\ \text { CONECT } & 51 & 50 & 44 & 52 & \\ \text { CONECT } & 52 & 51 & & & \\ \text { CONECT } & 53 & 48 & & & \\ \text { CONECT } & 54 & 45 & & & \\ \text { CONECT } & 55 & 43 & & & \\ \text { CONECT } & 56 & 50 & & & \\ \text { CONECT } & 57 & 50 & & & \\ & & & & & \end{array}$
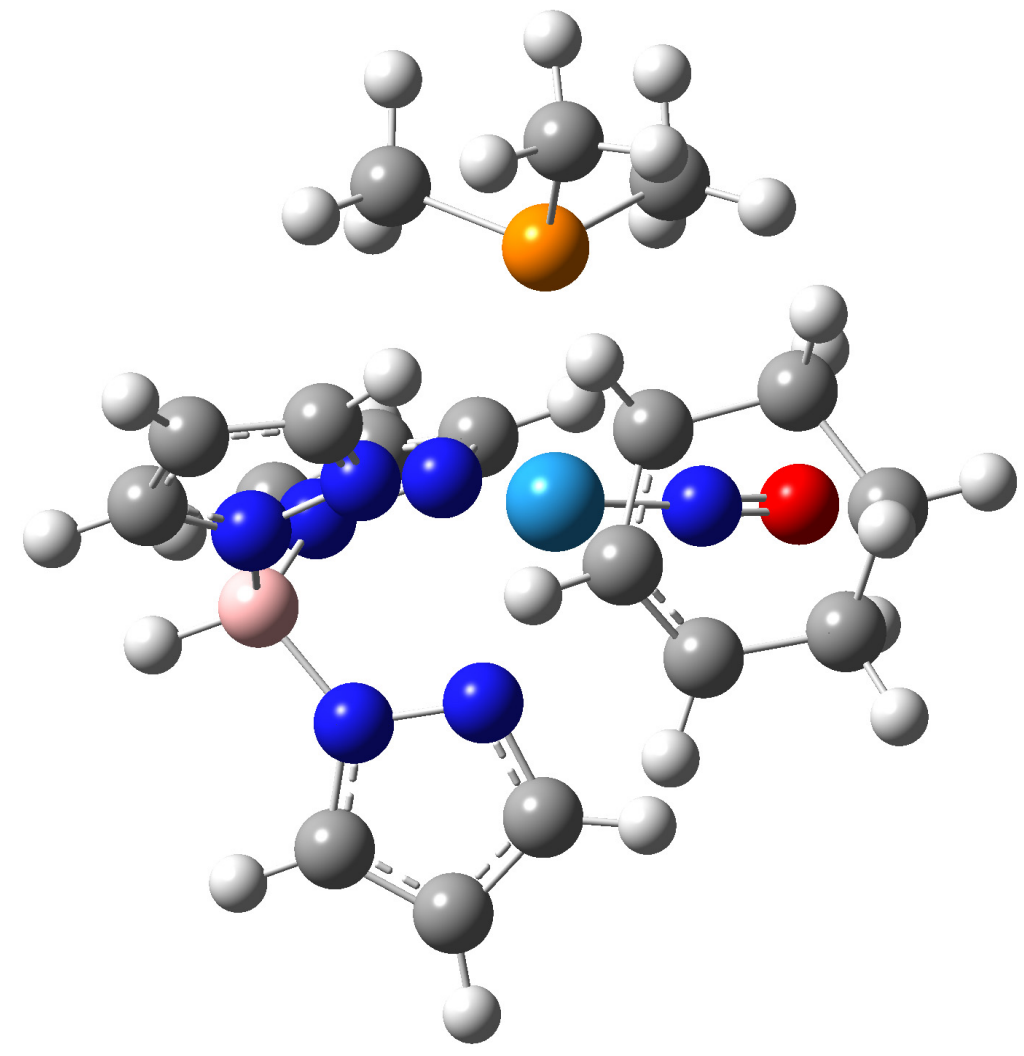


$\begin{array}{llllllll}\text { TITLE } & \text { Exo } & \text { 3p } & & & & & \\ \text { REMARK } & 1 & \text { File } & & & & & \\ \text { HETATM } & 1 & \mathrm{~W} & 0 & -0.419 & 0.151 & -0.044 & \mathrm{~W} \\ \text { HETATM } & 2 & \mathrm{~N} & 0 & -1.264 & 0.661 & -1.538 \mathrm{~N} \\ \text { HETATM } & 3 & \mathrm{O} & 0 & -1.748 & 0.951 & -2.59 & \mathrm{O} \\ \text { HETATM } & 4 & \mathrm{P} & 0 & -1.155 & -2.356 & -0.2 & \mathrm{P} \\ \text { HETATM } & 5 & \mathrm{C} & 0 & -2.245 & -2.755 & -1.634 & \mathrm{C} \\ \text { HETATM } & 6 & \mathrm{H} & 0 & -1.758 & -2.456 & -2.567 & \mathrm{H} \\ \text { HETATM } & 7 & \mathrm{H} & 0 & -2.458 & -3.828 & -1.674 & \mathrm{H} \\ \text { HETATM } & 8 & \mathrm{H} & 0 & -3.187 & -2.205 & -1.556 & \mathrm{H} \\ \text { HETATM } & 9 & \mathrm{C} & 0 & -2.043 & -3.12 & 1.232 & \mathrm{C} \\ \text { HETATM } & 10 & \mathrm{H} & 0 & -2.294 & -4.158 & 0.992 & \mathrm{H} \\ \text { HETATM } & 11 & \mathrm{H} & 0 & -1.392 & -3.12 & 2.111 & \mathrm{H} \\ \text { HETATM } & 12 & \mathrm{H} & 0 & -2.967 & -2.591 & 1.478 & \mathrm{H} \\ \text { HETATM } & 13 & \mathrm{C} & 0 & 0.257 & -3.531 & -0.374 \mathrm{C} \\ \text { HETATM } & 14 & \mathrm{H} & 0 & -0.118 & -4.56 & -0.371 & \mathrm{H} \\ \text { HETATM } & 15 & \mathrm{H} & 0 & 0.802 & -3.353 & -1.302 \mathrm{H} \\ \text { HETATM } & 16 & \mathrm{H} & 0 & 0.944 & -3.399 & 0.466 & \mathrm{H} \\ \text { HETATM } & 17 & \mathrm{~N} & 0 & 1.082 & 1.793 & -0.16 & \mathrm{~N} \\ \text { HETATM } & 39 & \mathrm{C} & 0 & 2.443 & -1.408 & -3.035 \mathrm{C} \\ \text { HETATM } & 40 & \mathrm{H} & 0 & 2.733 & -1.795 & -4.001 & \mathrm{H} \\ \text { HETATM } & 18 & \mathrm{~N} & 0 & 2.401 & 1.578 & 0.112 & \mathrm{~N} \\ \text { HETATM } & 41 & \mathrm{C} & 0 & 3.268 & -1.119 & -1.955 \mathrm{C} \\ \text { HETATM } & 19 & \mathrm{~N} & 0 & 1.205 & -0.62 & -1.332 \mathrm{~N} \\ \text { HETATM } & 20 & \mathrm{~N} & 0 & 2.508 & -0.653 & -0.941 & \mathrm{~N} \\ \text { HETATM } & 36 & \mathrm{H} & 0 & 4.339 & -1.196 & -1.842 \mathrm{H}\end{array}$




\begin{tabular}{|c|c|c|c|c|c|c|}
\hline HETATM & 43 & C & 0 & -2.036 & 0.438 & $1.609 \mathrm{C}$ \\
\hline HETATM & 44 & $\mathrm{H}$ & 0 & -1.86 & -0.315 & 2.368 \\
\hline HETATM & 45 & C & 0 & -3.04 & 0.238 & $0.671 \mathrm{c}$ \\
\hline HETATM & 46 & $\mathrm{H}$ & 0 & -3.577 & -0.708 & 0.674 \\
\hline HETATM & 47 & C & 0 & -1.24 & 1.631 & 1.478 \\
\hline HETATM & 48 & $\mathrm{H}$ & 0 & -0.495 & 1.782 & 2.257 \\
\hline HETATM & 49 & C & 0 & -2 & 2.898 & 1.091 \\
\hline HETATM & 50 & $\mathrm{H}$ & 0 & -1.305 & 3.716 & 0.88 \\
\hline HETATM & 51 & $\mathrm{H}$ & 0 & -2.565 & 3.215 & 1.98 \\
\hline HETATM & 52 & C & 0 & -3.71 & 1.355 & -0.08 \\
\hline HETATM & 53 & $\mathrm{H}$ & 0 & -4.685 & 1.46 & 0.425 \\
\hline HETATM & 54 & $\mathrm{H}$ & 0 & -3.953 & 1.043 & -1.103 \\
\hline HETATM & 55 & C & 0 & -2.958 & 2.705 & $-0.097 \mathrm{C}$ \\
\hline HETATM & 56 & $\mathrm{H}$ & 0 & -3.69 & 3.519 & -0.115 \\
\hline HETATM & 57 & $\mathrm{H}$ & 0 & -2.401 & 2.78 & -1.034 \\
\hline \multicolumn{7}{|l|}{ END } \\
\hline CONECT & 1 & 2 & & & & \\
\hline CONECT & 2 & 1 & 3 & & & \\
\hline CONECT & 3 & 2 & & & & \\
\hline CONECT & 4 & 5 & 9 & 13 & & \\
\hline CONECT & 5 & 4 & 6 & 7 & 8 & \\
\hline CONECT & 6 & 5 & & & & \\
\hline CONECT & 7 & 5 & & & & \\
\hline CONECT & 8 & 5 & & & & \\
\hline CONECT & 9 & 4 & 10 & 11 & 12 & \\
\hline CONECT & 10 & 9 & & & & \\
\hline CONECT & 11 & 9 & & & & \\
\hline CONECT & 12 & 9 & & & & \\
\hline CONECT & 13 & 4 & 14 & 15 & 16 & \\
\hline CONECT & 14 & 13 & & & & \\
\hline CONECT & 15 & 13 & & & & \\
\hline CONECT & 16 & 13 & & & & \\
\hline CONECT & 17 & 18 & 25 & & & \\
\hline CONECT & 18 & 17 & 23 & 29 & & \\
\hline CONECT & 19 & 20 & 37 & & & \\
\hline CONECT & 20 & 19 & 23 & 41 & & \\
\hline CONECT & 21 & 22 & 31 & & & \\
\hline CONECT & 22 & 21 & 23 & 35 & & \\
\hline CONECT & 23 & 18 & 20 & 22 & 24 & \\
\hline CONECT & 24 & 23 & & & & \\
\hline CONECT & 25 & 17 & 26 & 27 & & \\
\hline CONECT & 26 & 25 & & & & \\
\hline CONECT & 27 & 25 & 28 & 29 & & \\
\hline CONECT & 28 & 27 & & & & \\
\hline
\end{tabular}




$\begin{array}{llllll}\text { CONECT } & 29 & 18 & 27 & 30 \\ \text { CONECT } & 30 & 29 & & & \\ \text { CONECT } & 31 & 21 & 32 & 33 & \\ \text { CONECT } & 32 & 31 & & & \\ \text { CONECT } & 33 & 31 & 34 & 35 & \\ \text { CONECT } & 34 & 33 & & & \\ \text { CONECT } & 35 & 22 & 33 & 36 & \\ \text { CONECT } & 36 & 35 & & & \\ \text { CONECT } & 37 & 19 & 38 & 39 & \\ \text { CONECT } & 38 & 37 & & & \\ \text { CONECT } & 39 & 37 & 40 & 41 & \\ \text { CONECT } & 40 & 39 & & & \\ \text { CONECT } & 41 & 39 & 20 & 42 & \\ \text { CONECT } & 42 & 41 & & & \\ \text { CONECT } & 43 & 44 & 45 & 47 & \\ \text { CONECT } & 44 & 43 & & & \\ \text { CONECT } & 45 & 43 & 46 & 52 & \\ \text { CONECT } & 46 & 45 & & & \\ \text { CONECT } & 47 & 43 & 48 & 49 & \\ \text { CONECT } & 48 & 47 & & & \\ \text { CONECT } & 49 & 47 & 50 & 51 \\ \text { CONECT } & 50 & 49 & & & \\ \text { CONECT } & 51 & 49 & & & \\ \text { CONECT } & 52 & 45 & 53 & 54 \\ \text { CONECT } & 53 & 52 & & & \\ \text { CONECT } & 54 & 52 & & & \\ \text { CONECT } & 55 & 49 & 52 & 56 & \\ \text { CONECT } & 56 & 55 & & & \\ \text { CONECT } & 57 & 55 & & & \\ & & & \end{array}$




\begin{tabular}{|c|c|c|c|c|c|c|c|}
\hline \multirow{3}{*}{$\begin{array}{l}\text { TITLE } \\
\text { REMARK } \\
\text { HETATM }\end{array}$} & \multicolumn{7}{|c|}{ Exo 3ts } \\
\hline & \multirow{2}{*}{$\begin{array}{l}1 \\
1\end{array}$} & \multicolumn{6}{|c|}{ File created by GaussView 5.0.8 } \\
\hline & & B & 0 & 2.964 & -0.199 & 0.329 & $B$ \\
\hline HETATM & 2 & $\mathrm{~N}$ & 0 & 2.445 & -0.862 & -0.972 & $\mathrm{~N}$ \\
\hline HETATM & 3 & $\mathrm{~N}$ & 0 & 2.615 & 1.299 & 0.198 & $\mathrm{~N}$ \\
\hline HETATM & 4 & $\mathrm{~N}$ & 0 & 2.227 & -0.797 & 1.539 & \\
\hline HETATM & 5 & $\mathrm{~N}$ & 0 & 0.87 & -0.721 & 1.653 & $\mathrm{~N}$ \\
\hline HETATM & 6 & $\mathrm{C}$ & 0 & 0.559 & -1.333 & 2.815 & $\mathrm{C}$ \\
\hline HETATM & 7 & C & 0 & 1.708 & -1.8 & 3.454 & $\mathrm{C}$ \\
\hline HETATM & 8 & C & 0 & 2.746 & -1.435 & 2.606 & C \\
\hline HETATM & 9 & C & 0 & 3.155 & -1.289 & -2.037 & C \\
\hline HETATM & 10 & C & 0 & 2.311 & -1.366 & -3.138 & C \\
\hline HETATM & 11 & C & 0 & 1.07 & -0.938 & -2.661 & C \\
\hline HETATM & 12 & $\mathrm{~N}$ & 0 & 1.151 & -0.648 & -1.349 & $\mathrm{~N}$ \\
\hline HETATM & 13 & C & 0 & 3.453 & 2.349 & 0.06 & C \\
\hline HETATM & 14 & C & 0 & 2.713 & 3.468 & -0.291 & C \\
\hline HETATM & 15 & C & 0 & 1.395 & 3.01 & -0.358 & C \\
\hline HETATM & 16 & $\mathrm{~N}$ & 0 & 1.335 & 1.702 & -0.056 & $\mathrm{~N}$ \\
\hline HETATM & 17 & W & 0 & -0.366 & 0.236 & -0.012 & \\
\hline HETATM & 18 & $P$ & 0 & -1.43 & -2.142 & -0.279 & $\mathrm{P}$ \\
\hline HETATM & 19 & C & 0 & -1.98 & -3.116 & 1.197 & C \\
\hline HETATM & 20 & C & 0 & -2.897 & -2.176 & -1.395 & C \\
\hline HETATM & 21 & C & 0 & -0.295 & -3.392 & -1.031 & C \\
\hline HETATM & 22 & $N$ & 0 & -1.169 & 0.762 & -1.519 & $\mathrm{~N}$ \\
\hline HETATM & 23 & $\mathrm{O}$ & 0 & -1.609 & 0.972 & -2.611 & $\mathrm{O}$ \\
\hline HETATM & 24 & C & 0 & -1.138 & 2.141 & 1.258 & C \\
\hline HETATM & 25 & C & 0 & -1.664 & 0.961 & 1.818 & C \\
\hline HETATM & 26 & C & 0 & -2.09 & 3.114 & 0.58 & C \\
\hline HETATM & 27 & C & 0 & -3.245 & 2.462 & -0.206 & C \\
\hline HETATM & 28 & C & 0 & -3.714 & 1.093 & 0.335 & C \\
\hline HETATM & 29 & C & 0 & -2.691 & 0.309 & 1.132 & C \\
\hline HETATM & 30 & $\mathrm{H}$ & 0 & 4.144 & -0.356 & 0.444 & $\mathrm{H}$ \\
\hline HETATM & 31 & $\mathrm{H}$ & 0 & -0.467 & -1.411 & 3.143 & $\mathrm{H}$ \\
\hline HETATM & 32 & $\mathrm{H}$ & 0 & 1.777 & -2.319 & 4.398 & $\mathrm{H}$ \\
\hline HETATM & 33 & $\mathrm{H}$ & 0 & 3.813 & -1.581 & 2.689 & $\mathrm{H}$ \\
\hline HETATM & 34 & $\mathrm{H}$ & 0 & 4.212 & -1.492 & -1.94 & $\mathrm{H}$ \\
\hline HETATM & 35 & $\mathrm{H}$ & 0 & 2.563 & -1.675 & -4.141 & $\mathrm{H}$ \\
\hline HETATM & 36 & $\mathrm{H}$ & 0 & 0.138 & -0.811 & -3.193 & $\mathrm{H}$ \\
\hline HETATM & 37 & $\mathrm{H}$ & 0 & 4.515 & 2.221 & 0.215 & $\mathrm{H}$ \\
\hline HETATM & 38 & $\mathrm{H}$ & 0 & 3.075 & 4.468 & -0.475 & $\mathrm{H}$ \\
\hline HETATM & 39 & $\mathrm{H}$ & 0 & 0.496 & 3.555 & -0.603 & $\mathrm{H}$ \\
\hline HETATM & 40 & $\mathrm{H}$ & 0 & -2.708 & -2.586 & 1.814 & H \\
\hline HETATM & 41 & $\mathrm{H}$ & 0 & -1.111 & -3.364 & 1.813 & $\mathrm{H}$ \\
\hline HETATM & 42 & $\mathrm{H}$ & 0 & -2.438 & -4.049 & 0.853 & $\mathrm{H}$ \\
\hline
\end{tabular}




\begin{tabular}{|c|c|c|c|c|c|c|}
\hline HETATM & 43 & $\mathrm{H}$ & 0 & -2.693 & -1.587 & -2.295 \\
\hline HETATM & 44 & $\mathrm{H}$ & 0 & -3.764 & -1.739 & -0.893 \\
\hline HETATM & 45 & $\mathrm{H}$ & 0 & -3.137 & -3.205 & -1.68 \\
\hline HETATM & 46 & $\mathrm{H}$ & 0 & -0.068 & -3.149 & -2.069 \\
\hline HETATM & 47 & $\mathrm{H}$ & 0 & -0.771 & -4.378 & -0.992 \\
\hline HETATM & 48 & $\mathrm{H}$ & 0 & 0.644 & -3.426 & -0.471 \\
\hline HETATM & 49 & $\mathrm{H}$ & 0 & -3.047 & -0.628 & 1.545 \\
\hline HETATM & 50 & $\mathrm{H}$ & 0 & -1.193 & 0.502 & 2.679 \\
\hline HETATM & 51 & $\mathrm{H}$ & 0 & -0.304 & 2.595 & 1.784 \\
\hline HETATM & 52 & $\mathrm{H}$ & 0 & -1.552 & 3.806 & $-0.077 \vdash$ \\
\hline HETATM & 53 & $\mathrm{H}$ & 0 & -2.49 & 3.739 & 1.393 \\
\hline HETATM & 54 & $\mathrm{H}$ & 0 & -4.118 & 0.493 & -0.487 \\
\hline HETATM & 55 & $\mathrm{H}$ & 0 & -4.555 & 1.229 & 1.034 \\
\hline HETATM & 56 & $\mathrm{H}$ & 0 & -4.1 & 3.147 & -0.216 \\
\hline HETATM & 57 & $\mathrm{H}$ & 0 & -2.948 & 2.347 & -1.248 \\
\hline \multicolumn{7}{|l|}{ END } \\
\hline CONECT & 1 & 2 & 3 & 4 & 30 & \\
\hline CONECT & 2 & 1 & 9 & 12 & & \\
\hline CONECT & 3 & 1 & 13 & 16 & & \\
\hline CONECT & 4 & 1 & 5 & 8 & & \\
\hline CONECT & 5 & 4 & 6 & & & \\
\hline CONECT & 6 & 5 & 7 & 31 & & \\
\hline CONECT & 7 & 6 & 8 & 32 & & \\
\hline CONECT & 8 & 7 & 4 & 33 & & \\
\hline CONECT & 9 & 2 & 10 & 34 & & \\
\hline CONECT & 10 & 9 & 11 & 35 & & \\
\hline CONECT & 11 & 10 & 12 & 36 & & \\
\hline CONECT & 12 & 11 & 2 & & & \\
\hline CONECT & 13 & 3 & 14 & 37 & & \\
\hline CONECT & 14 & 13 & 15 & 38 & & \\
\hline CONECT & 15 & 14 & 16 & 39 & & \\
\hline CONECT & 16 & 3 & 15 & & & \\
\hline CONECT & 17 & 22 & & & & \\
\hline CONECT & 18 & 19 & 20 & 21 & & \\
\hline CONECT & 19 & 18 & 40 & 41 & 42 & \\
\hline CONECT & 20 & 18 & 43 & 44 & 45 & \\
\hline CONECT & 21 & 18 & 46 & 47 & 48 & \\
\hline CONECT & 22 & 17 & 23 & & & \\
\hline CONECT & 23 & 22 & & & & \\
\hline CONECT & 24 & 25 & 26 & 51 & & \\
\hline CONECT & 25 & 24 & 29 & 50 & & \\
\hline CONECT & 26 & 24 & 27 & 52 & 53 & \\
\hline CONECT & 27 & 26 & 28 & 56 & 57 & \\
\hline CONECT & 28 & 27 & 29 & 54 & 55 & \\
\hline
\end{tabular}




$\begin{array}{lllll}\text { CONECT } & 29 & 25 & 28 & 49 \\ \text { CONECT } & 30 & 1 & & \\ \text { CONECT } & 31 & 6 & & \\ \text { CONECT } & 32 & 7 & & \\ \text { CONECT } & 33 & 8 & & \\ \text { CONECT } & 34 & 9 & \\ \text { CONECT } & 35 & 10 & \\ \text { CONECT } & 36 & 11 & \\ \text { CONECT } & 37 & 13 & \\ \text { CONECT } & 38 & 14 & \\ \text { CONECT } & 39 & 15 & \\ \text { CONECT } & 40 & 19 & \\ \text { CONECT } & 41 & 19 & \\ \text { CONECT } & 42 & 19 & \\ \text { CONECT } & 43 & 20 & \\ \text { CONECT } & 44 & 20 & \\ \text { CONECT } & 45 & 20 & \\ \text { CONECT } & 46 & 21 & \\ \text { CONECT } & 47 & 21 & \\ \text { CONECT } & 48 & 21 & \\ \text { CONECT } & 49 & 29 & \\ \text { CONECT } & 50 & 25 & \\ \text { CONECT } & 51 & 24 & \\ \text { CONECT } & 52 & 26 & \\ \text { CONECT } & 53 & 26 & \\ \text { CONECT } & 54 & 28 \\ \text { CONECT } & 55 & 28 \\ \text { CONECT } & 56 & 27 \\ \text { CONECT } & 57 & 27 & \\ & & & \end{array}$
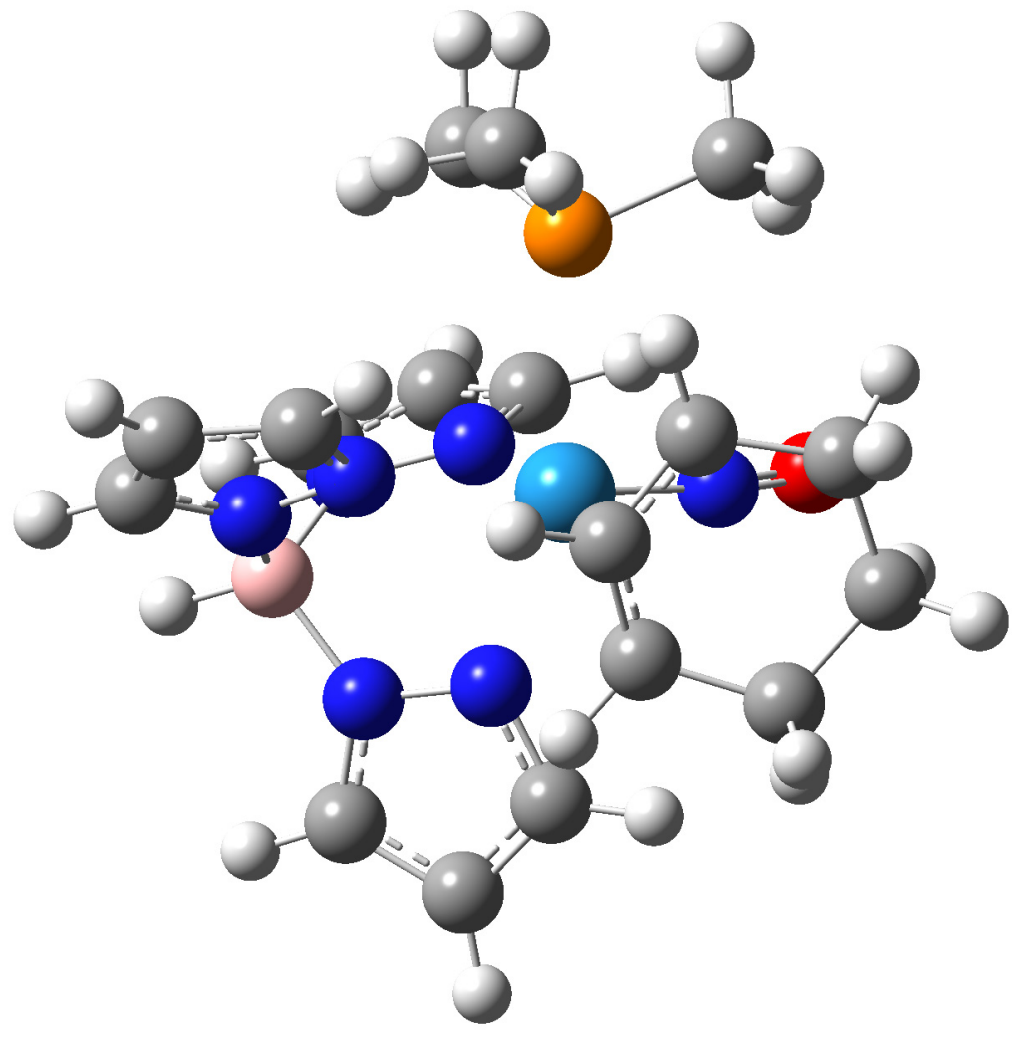
TITLE

HETATM

HETATM

HETATM

HETATM

HETATM

HETATM

HETATM

HETATM

HETATM

HETATM

HETATM

HETATM

HETATM

HETATM

HETATM

HETATM

HETATM

HETATM

HETATM

HETATIM

HETATM

HETATM 22

HETATM 23

HETATM 24

HETATM 25

HETATM 26

HETATM 27 W

$\begin{array}{llll}\text { HETATM } & 28 & P & 0\end{array}$

HETATM 29 C 0

$\begin{array}{llll}\text { HETATM } & 30 & \mathrm{H} & 0\end{array}$

$\begin{array}{llll}\text { HETATM } & 31 & \mathrm{H} & 0\end{array}$

$\begin{array}{llll}\text { HETATM } & 32 & \mathrm{H} & 0\end{array}$

HETATM 33 C 0

$\begin{array}{llll}\text { HETATM } & 34 & \mathrm{H} & 0\end{array}$

HETATM 35 H 0

HETATM 36 H 0

$\begin{array}{llll}\text { HETATM } & 37 & \mathrm{C} & 0\end{array}$

$\begin{array}{llll}\text { HETATM } & 38 & \mathrm{H} & 0\end{array}$

HETATM $39 \quad \mathrm{H} \quad 0$

HETATM $40 \quad \mathrm{H} \quad 0$

HETATM 41 N 0

$\begin{array}{llll}\text { HETATM } & 42 & 0 & 0\end{array}$ $\begin{array}{llll}-2.48 & -0.212 & 1.41 & B\end{array}$

$\begin{array}{llll}-2.291 & 1.22 & 0.843 \quad \mathrm{~N}\end{array}$

$\begin{array}{llll}-2.623 & -1.138 & 0.18 \quad \mathrm{~N}\end{array}$

$\begin{array}{lll}-1.234 & -0.584 & 2.225 \mathrm{~N}\end{array}$

$-3.459-0.2692 .096 \mathrm{H}$

$-1.2981 .453-0.063 \mathrm{~N}$

$\begin{array}{lll}-1.594 & 2.614 & -0.676 \mathrm{C}\end{array}$

$-2.7683 .156-0.149 \mathrm{C}$

$\begin{array}{llll}-3.183 & 2.231 & 0.8 & C\end{array}$

$-1.159-0.8163 .551 \quad C$

$\begin{array}{lllll}0.177 & -0.945 & 3.904 \quad C\end{array}$

$\begin{array}{lllll}0.869 & -0.769 & 2.705 \quad C\end{array}$

$\begin{array}{lllll}0.019 & -0.553 & 1.681 & N\end{array}$

$-3.724-1.781-0.264 \mathrm{C}$

$-3.5 \quad-2.205-1.567 \mathrm{C}$

$-2.21 \quad-1.76-1.864 \mathrm{C}$

$-1.679-1.125-0.804 \mathrm{~N}$

$-0.9632 .991-1.468 \mathrm{H}$

$-3.2544 .079-0.428 \mathrm{H}$

$\begin{array}{llll}-4.057 & 2.212 & 1.435 \quad \mathrm{H}\end{array}$

$\begin{array}{llll}-2.058 & -0.87 & 4.147 \quad \mathrm{H}\end{array}$

$0.587-1.142 \quad 4.883 \quad \mathrm{H}$

$\begin{array}{llll}1.932 & -0.803 & 2.536 \mathrm{H}\end{array}$

$\begin{array}{lllll}-4.59 & -1.881 & 0.374 \quad H\end{array}$

$-4.176-2.749-2.209 \mathrm{H}$

$-1.644-1.861-2.78 \quad H$

$0.255-0.063-0.547 \mathrm{~W}$

$\begin{array}{llll}1.733 & 1.94 & 0.259 & P\end{array}$

$\begin{array}{lllll}3.399 & 1.68 & 1.015 \quad C\end{array}$

$\begin{array}{llll}4.06 & 1.125 & 0.347 & \mathrm{H}\end{array}$

$\begin{array}{lllll}3.314 & 1.141 & 1.961 \quad \mathrm{H}\end{array}$

$\begin{array}{lllll}3.847 & 2.659 & 1.218 \quad \mathrm{H}\end{array}$

$2.137 \quad 3.084 \quad-1.131 \mathrm{C}$

$\begin{array}{lll}1.228 & 3.45 & -1.615 \mathrm{H}\end{array}$

$2.725 \quad 2.551 \quad-1.884 \mathrm{H}$

$\begin{array}{llll}2.713 & 3.941 & -0.767 \mathrm{H}\end{array}$

$\begin{array}{lllll}0.958 & 3.019 & 1.542 & \mathrm{C}\end{array}$

$\begin{array}{lllll}0.047 & 3.488 & 1.168 \quad \mathrm{H}\end{array}$

$\begin{array}{llll}1.665 & 3.799 & 1.842 \quad \mathrm{H}\end{array}$

$\begin{array}{lllll}0.703 & 2.414 & 2.417 & H\end{array}$

$\begin{array}{lllll}0.247 & 0.563 & -2.22 & \mathrm{~N}\end{array}$

$\begin{array}{llll}0.2 & 1.032 & -3.314 & 0\end{array}$ 


\begin{tabular}{|c|c|c|c|c|c|}
\hline HETATM & 43 & C & 0 & 1.601 & $-1.685-1.674$ \\
\hline HETATM & 44 & C & 0 & 2.463 & $-0.867-0.865$ \\
\hline HETATM & 45 & C & 0 & 0.8 & $-2.602-1.009$ \\
\hline HETATM & 46 & $\mathrm{H}$ & 0 & 0.012 & $-3.104-1.56$ \\
\hline HETATM & 47 & $\mathrm{H}$ & 0 & 1.476 & $-1.506-2.737$ \\
\hline HETATM & 48 & C & 0 & 3.229 & -1.5840 .26 \\
\hline HETATM & 49 & $\mathrm{H}$ & 0 & 4.293 & $-1.583-0.011$ \\
\hline HETATM & 50 & $\mathrm{H}$ & 0 & 3.181 & -1.0331 .2 \\
\hline HETATM & 51 & C & 0 & 1.259 & -3.2450 .283 \\
\hline HETATM & 52 & $\mathrm{H}$ & 0 & 0.699 & -2.8851 .15 \\
\hline HETATM & 53 & $\mathrm{H}$ & 0 & 1.032 & -4.3160 .209 \\
\hline HETATM & 54 & C & 0 & 2.776 & -3.0430 .458 \\
\hline HETATM & 55 & $\mathrm{H}$ & 0 & 3.097 & -3.4111 .438 \\
\hline HETATM & 56 & $\mathrm{H}$ & 0 & 3.281 & $-3.665-0.291$ \\
\hline HETATM & 57 & $\mathrm{H}$ & 0 & 3.064 & $-0.158-1.432$ \\
\hline \multicolumn{6}{|l|}{ END } \\
\hline CONECT & 1 & 2 & 3 & 4 & 5 \\
\hline CONECT & 2 & 1 & 6 & 9 & \\
\hline CONECT & 3 & 1 & 14 & 17 & \\
\hline CONECT & 4 & 1 & 10 & 13 & \\
\hline CONECT & 5 & 1 & & & \\
\hline CONECT & 6 & 2 & 7 & & \\
\hline CONECT & 7 & 6 & 8 & 18 & \\
\hline CONECT & 8 & 7 & 9 & 19 & \\
\hline CONECT & 9 & 2 & 8 & 20 & \\
\hline CONECT & 10 & 4 & 11 & 21 & \\
\hline CONECT & 11 & 10 & 12 & 22 & \\
\hline CONECT & 12 & 11 & 13 & 23 & \\
\hline CONECT & 13 & 4 & 12 & & \\
\hline CONECT & 14 & 3 & 15 & 24 & \\
\hline CONECT & 15 & 14 & 16 & 25 & \\
\hline CONECT & 16 & 15 & 17 & 26 & \\
\hline CONECT & 17 & 16 & 3 & & \\
\hline CONECT & 18 & 7 & & & \\
\hline CONECT & 19 & 8 & & & \\
\hline CONECT & 20 & 9 & & & \\
\hline CONECT & 21 & 10 & & & \\
\hline CONECT & 22 & 11 & & & \\
\hline CONECT & 23 & 12 & & & \\
\hline CONECT & 24 & 14 & & & \\
\hline CONECT & 25 & 15 & & & \\
\hline CONECT & 26 & 16 & & & \\
\hline CONECT & 27 & 41 & & & \\
\hline CONECT & 28 & 29 & 33 & 37 & \\
\hline
\end{tabular}




\begin{tabular}{|c|c|c|c|c|}
\hline CONECT & 29 & 28 & 30 & 31 \\
\hline CONECT & 30 & 29 & & \\
\hline CONECT & 31 & 29 & & \\
\hline CONECT & 32 & 29 & & \\
\hline CONECT & 33 & 28 & 34 & 35 \\
\hline CONECT & 34 & 33 & & \\
\hline CONECT & 35 & 33 & & \\
\hline CONECT & 36 & 33 & & \\
\hline CONECT & 37 & 28 & 38 & 39 \\
\hline CONECT & 38 & 37 & & \\
\hline CONECT & 39 & 37 & & \\
\hline CONECT & 40 & 37 & & \\
\hline CONECT & 41 & 27 & 42 & \\
\hline CONECT & 42 & 41 & & \\
\hline CONECT & 43 & 44 & 45 & 47 \\
\hline CONECT & 44 & 43 & 48 & 57 \\
\hline CONECT & 45 & 43 & 46 & 51 \\
\hline CONECT & 46 & 45 & & \\
\hline CONECT & 47 & 43 & & \\
\hline CONECT & 48 & 44 & 49 & 50 \\
\hline CONECT & 49 & 48 & & \\
\hline CONECT & 50 & 48 & & \\
\hline CONECT & 51 & 45 & 52 & 53 \\
\hline CONECT & 52 & 51 & & \\
\hline CONECT & 53 & 51 & & \\
\hline CONECT & 54 & 48 & 51 & 55 \\
\hline CONECT & 55 & 54 & & \\
\hline CONECT & 56 & 54 & & \\
\hline CONECT & 57 & 44 & & \\
\hline
\end{tabular}




\begin{tabular}{|c|c|c|c|c|c|c|c|}
\hline TITLE & Exo-5 & & & & & & \\
\hline REMARK & 1 & File & eat & y Gauss & View $5 .($ & 0.8 & \\
\hline HETATM & 1 & W & 0 & 0.014 & 0.143 & -0.118 & W \\
\hline HETATM & 2 & $\mathrm{~N}$ & 0 & 1.176 & 0.727 & 1.111 & $\mathrm{~N}$ \\
\hline HETATM & 3 & 0 & 0 & 1.868 & 1.105 & 2.006 & 0 \\
\hline HETATM & 4 & $P$ & 0 & 0.675 & -2.335 & 0.389 & $P$ \\
\hline HETATM & 5 & C & 0 & 2.011 & -2.479 & 1.648 & $C$ \\
\hline HETATM & 6 & $\mathrm{H}$ & 0 & 1.712 & -1.979 & 2.574 & $\mathrm{H}$ \\
\hline HETATM & 7 & $\mathrm{H}$ & 0 & 2.21 & -3.534 & 1.866 & $\mathrm{H}$ \\
\hline HETATM & 8 & $\mathrm{H}$ & 0 & 2.928 & -2.004 & 1.285 & $\mathrm{H}$ \\
\hline HETATM & 9 & C & 0 & 1.264 & -3.44 & -0.971 & $\mathrm{C}$ \\
\hline HETATM & 10 & $\mathrm{H}$ & 0 & 1.438 & -4.44 & -0.56 & $\mathrm{H}$ \\
\hline HETATM & 11 & $\mathrm{H}$ & 0 & 0.504 & -3.517 & -1.753 & $\mathrm{H}$ \\
\hline HETATM & 12 & $\mathrm{H}$ & 0 & 2.198 & -3.085 & -1.41 & $\mathrm{H}$ \\
\hline HETATM & 13 & C & 0 & -0.707 & -3.373 & 1.044 & C \\
\hline HETATM & 14 & $\mathrm{H}$ & 0 & -0.352 & -4.398 & 1.189 & $\mathrm{H}$ \\
\hline HETATM & 15 & $\mathrm{H}$ & 0 & -1.08 & -2.991 & 1.995 & $\mathrm{H}$ \\
\hline HETATM & 16 & $\mathrm{H}$ & 0 & -1.529 & -3.382 & 0.323 & $\mathrm{H}$ \\
\hline HETATM & 17 & $\mathrm{~N}$ & 0 & -1.343 & 1.918 & -0.053 & $\mathrm{~N}$ \\
\hline HETATM & 18 & $\mathrm{~N}$ & 0 & -2.699 & 1.747 & -0.064 & $\mathrm{~N}$ \\
\hline HETATM & 19 & $\mathrm{~N}$ & 0 & -1.377 & -0.323 & 1.521 & $\mathrm{~N}$ \\
\hline HETATM & 20 & $\mathrm{~N}$ & 0 & -2.731 & -0.277 & 1.358 & $\mathrm{~N}$ \\
\hline HETATM & 21 & $\mathrm{~N}$ & 0 & -1.614 & -0.715 & -1.46 & $\mathrm{~N}$ \\
\hline HETATM & 22 & $N$ & 0 & -2.926 & -0.514 & -1.147 & $\mathrm{~N}$ \\
\hline HETATM & 23 & B & 0 & -3.328 & 0.338 & 0.065 & B \\
\hline HETATM & 24 & $\mathrm{H}$ & 0 & -4.518 & 0.414 & 0.148 & $\mathrm{H}$ \\
\hline HETATM & 25 & $C$ & 0 & -1.124 & 3.236 & 0.114 & $C$ \\
\hline HETATM & 26 & $\mathrm{H}$ & 0 & -0.118 & 3.62 & 0.187 & $\mathrm{H}$ \\
\hline HETATM & 27 & C & 0 & -2.334 & 3.929 & 0.19 & $C$ \\
\hline HETATM & 28 & $\mathrm{H}$ & 0 & -2.481 & 4.991 & 0.314 & $\mathrm{H}$ \\
\hline HETATM & 29 & C & 0 & -3.305 & 2.944 & 0.08 & $\mathrm{C}$ \\
\hline HETATM & 30 & $\mathrm{H}$ & 0 & -4.384 & 3.008 & 0.103 & $\mathrm{H}$ \\
\hline HETATM & 31 & $C$ & 0 & -1.608 & -1.455 & -2.587 & $C$ \\
\hline HETATM & 32 & $\mathrm{H}$ & 0 & -0.679 & -1.745 & -3.053 & $\mathrm{H}$ \\
\hline HETATM & 33 & C & 0 & -2.911 & -1.735 & -3.003 & $C$ \\
\hline HETATM & 34 & $\mathrm{H}$ & 0 & -3.222 & -2.301 & -3.868 & $\mathrm{H}$ \\
\hline HETATM & 35 & $C$ & 0 & -3.717 & -1.116 & -2.058 & $C$ \\
\hline HETATM & 36 & $\mathrm{H}$ & 0 & -4.792 & -1.058 & -1.971 & $\mathrm{H}$ \\
\hline HETATM & 37 & $\mathrm{C}$ & 0 & -1.145 & -0.543 & 2.829 & $\mathrm{C}$ \\
\hline HETATM & 38 & $\mathrm{H}$ & 0 & -0.132 & -0.596 & 3.201 & $\mathrm{H}$ \\
\hline HETATM & 39 & $C$ & 0 & -2.352 & -0.663 & 3.52 & $C$ \\
\hline HETATM & 40 & $\mathrm{H}$ & 0 & -2.495 & -0.846 & 4.574 & $\mathrm{H}$ \\
\hline HETATM & 41 & $C$ & 0 & -3.33 & -0.475 & 2.55 & $C$ \\
\hline HETATM & 42 & $\mathrm{H}$ & 0 & -4.407 & -0.453 & 2.628 & $\mathrm{H}$ \\
\hline
\end{tabular}




\begin{tabular}{|c|c|c|c|c|c|c|}
\hline HETATM & 43 & $C$ & 0 & 1.468 & -0.378 & -1.797 \\
\hline HETATM & 44 & $\mathrm{H}$ & 0 & 1.173 & -1.205 & -2.437 \\
\hline HETATM & 45 & $C$ & 0 & 0.895 & 0.898 & -2.146 \\
\hline HETATM & 46 & $\mathrm{H}$ & 0 & 0.033 & 1.001 & -2.799 \\
\hline HETATM & 47 & C & 0 & 1.421 & 2.006 & -1.512 \\
\hline HETATM & 48 & $\mathrm{H}$ & 0 & 0.944 & 2.971 & -1.651 \\
\hline HETATM & 49 & C & 0 & 2.974 & -0.361 & -1.553 \\
\hline HETATM & 50 & $\mathrm{H}$ & 0 & 3.333 & -1.263 & -1.058 \\
\hline HETATM & 51 & $\mathrm{H}$ & 0 & 3.476 & -0.313 & -2.534 \\
\hline HETATM & 52 & C & 0 & 2.819 & 2.061 & -0.95 \\
\hline HETATM & 53 & $\mathrm{H}$ & 0 & 2.826 & 2.635 & -0.018 \\
\hline HETATM & 54 & $\mathrm{H}$ & 0 & 3.397 & 2.657 & -1.682 \\
\hline HETATM & 55 & $N$ & 0 & 3.411 & 0.756 & -0.722 \\
\hline HETATM & 56 & $C$ & 0 & 4.375 & 0.511 & 0.245 \\
\hline HETATM & 57 & 0 & 0 & 4.738 & -0.636 & 0.49 \\
\hline HETATM & 58 & C & 0 & 4.969 & 1.703 & 0.97 \\
\hline HETATM & 59 & $\mathrm{H}$ & 0 & 5.246 & 2.514 & 0.288 \\
\hline HETATM & 60 & $\mathrm{H}$ & 0 & 4.263 & 2.098 & 1.709 \\
\hline HETATM & 61 & $\mathrm{H}$ & 0 & 5.859 & 1.356 & 1.496 \\
\hline \multicolumn{7}{|l|}{ END } \\
\hline CONECT & 1 & 2 & & & & \\
\hline CONECT & 2 & 1 & 3 & & & \\
\hline CONECT & 3 & 2 & & & & \\
\hline CONECT & 4 & 5 & 9 & 13 & & \\
\hline CONECT & 5 & 4 & 6 & 7 & 8 & \\
\hline CONECT & 6 & 5 & & & & \\
\hline CONECT & 7 & 5 & & & & \\
\hline CONECT & 8 & 5 & & & & \\
\hline CONECT & 9 & 4 & 10 & 11 & 12 & \\
\hline CONECT & 10 & 9 & & & & \\
\hline CONECT & 11 & 9 & & & & \\
\hline CONECT & 12 & 9 & & & & \\
\hline CONECT & 13 & 4 & 14 & 15 & 16 & \\
\hline CONECT & 14 & 13 & & & & \\
\hline CONECT & 15 & 13 & & & & \\
\hline CONECT & 16 & 13 & & & & \\
\hline CONECT & 17 & 18 & 25 & & & \\
\hline CONECT & 18 & 17 & 23 & 29 & & \\
\hline CONECT & 19 & 20 & 37 & & & \\
\hline CONECT & 20 & 19 & 23 & 41 & & \\
\hline CONECT & 21 & 22 & 31 & & & \\
\hline CONECT & 22 & 21 & 23 & 35 & & \\
\hline CONECT & 23 & 22 & 18 & 20 & 24 & \\
\hline CONECT & 24 & 23 & & & & \\
\hline
\end{tabular}




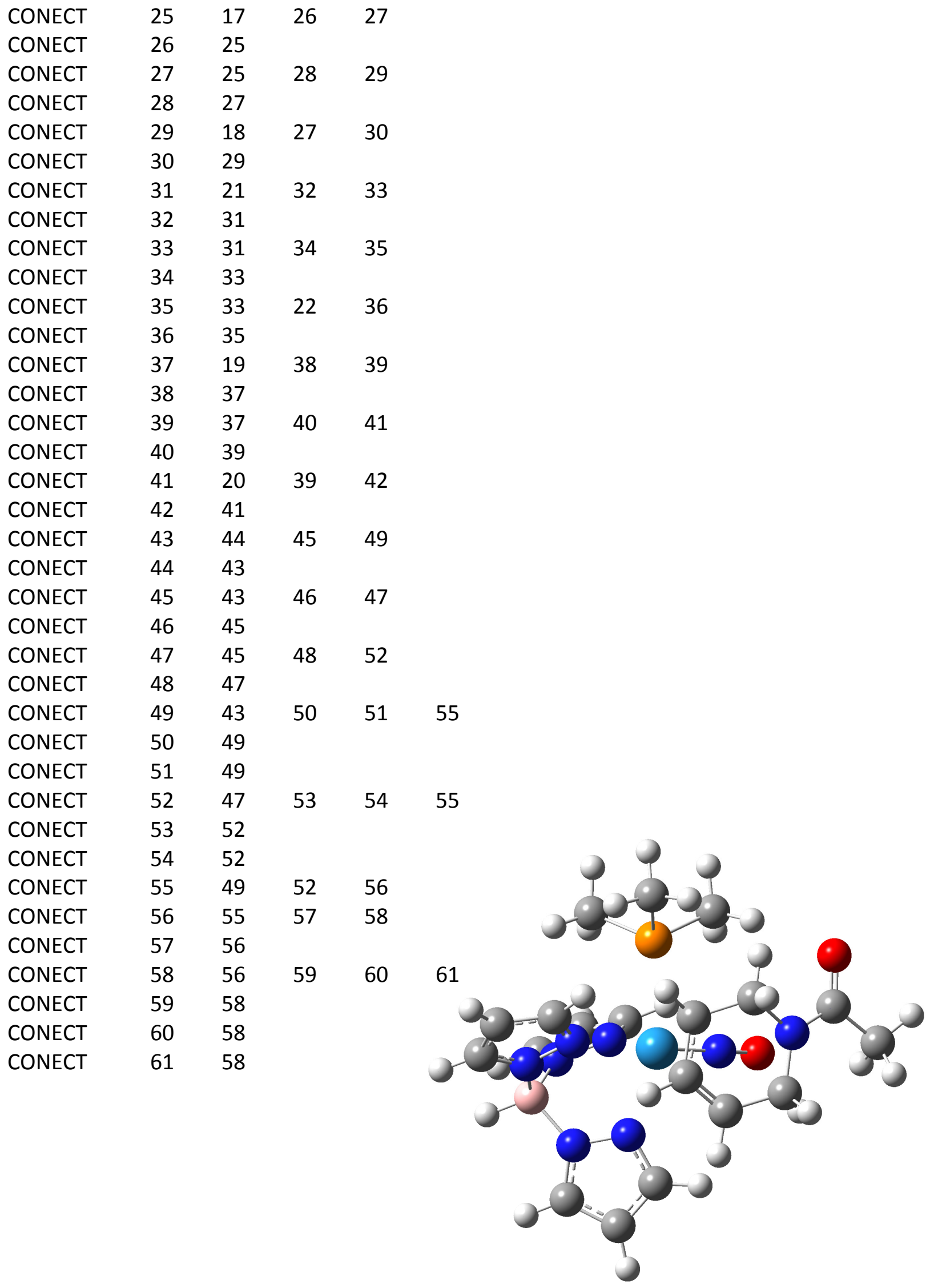




\begin{tabular}{|c|c|c|c|c|c|c|c|}
\hline TITLE & Exo & & & & & & \\
\hline REMARK & 1 & File & eat & y Gauss & View 5. & 0.8 & \\
\hline HETATM & 1 & $B$ & 0 & -3.365 & 0.438 & 0.035 & B \\
\hline HETATM & 2 & $\mathrm{~N}$ & 0 & -2.877 & -0.315 & 1.296 & $\mathrm{~N}$ \\
\hline HETATM & 3 & $\mathrm{~N}$ & 0 & -2.668 & 1.815 & 0.04 & $\mathrm{~N}$ \\
\hline HETATM & 4 & $\mathrm{~N}$ & 0 & -2.954 & -0.34 & -1.233 & $\mathrm{~N}$ \\
\hline HETATM & 5 & $\mathrm{H}$ & 0 & -4.558 & 0.568 & 0.064 & $\mathrm{H}$ \\
\hline HETATM & 6 & $\mathrm{~N}$ & 0 & -1.541 & -0.464 & 1.508 & $\mathrm{~N}$ \\
\hline HETATM & 7 & C & 0 & -1.389 & -0.845 & 2.784 & C \\
\hline HETATM & 8 & C & 0 & -2.637 & -0.964 & 3.411 & C \\
\hline HETATM & 9 & C & 0 & -3.552 & -0.608 & 2.43 & C \\
\hline HETATM & 10 & C & 0 & -3.743 & -0.817 & -2.219 & C \\
\hline HETATM & 11 & C & 0 & -2.938 & -1.399 & -3.187 & C \\
\hline HETATM & 12 & C & 0 & -1.635 & -1.229 & -2.703 & C \\
\hline HETATM & 13 & $\mathrm{~N}$ & 0 & -1.646 & -0.59 & -1.522 & $\mathrm{~N}$ \\
\hline HETATM & 14 & C & 0 & -3.209 & 3.046 & 0.185 & C \\
\hline HETATM & 15 & C & 0 & -2.181 & 3.967 & 0.312 & C \\
\hline HETATM & 16 & C & 0 & -1.009 & 3.2 & 0.238 & C \\
\hline HETATM & 17 & $N$ & 0 & -1.308 & 1.906 & 0.07 & $\mathrm{~N}$ \\
\hline HETATM & 18 & $\mathrm{H}$ & 0 & -0.396 & -0.993 & 3.186 & $\mathrm{H}$ \\
\hline HETATM & 19 & $\mathrm{H}$ & 0 & -2.842 & -1.255 & 4.43 & 1 \\
\hline HETATM & 20 & $\mathrm{H}$ & 0 & -4.629 & -0.528 & 2.459 & $\mathrm{H}$ \\
\hline HETATM & 21 & $\mathrm{H}$ & 0 & -4.816 & -0.704 & -2.16 & $\mathrm{H}$ \\
\hline HETATM & 22 & $\mathrm{H}$ & 0 & -3.247 & -1.872 & -4.107 & $\mathrm{H}$ \\
\hline HETATM & 23 & $\mathrm{H}$ & 0 & -0.699 & -1.525 & -3.151 & $\mathrm{H}$ \\
\hline HETATM & 24 & $\mathrm{H}$ & 0 & -4.282 & 3.172 & 0.189 & $\mathrm{H}$ \\
\hline HETATM & 25 & $\mathrm{H}$ & 0 & -2.265 & 5.036 & 0.442 & $\Pi$ \\
\hline HETATM & 26 & $\mathrm{H}$ & 0 & 0.025 & 3.503 & 0.292 & $\mathrm{H}$ \\
\hline HETATM & 27 & W & 0 & -0.002 & 0.072 & -0.051 & n \\
\hline HETATM & 28 & $P$ & 0 & 0.722 & -2.364 & 0.177 & $\mathrm{P}$ \\
\hline HETATM & 29 & C & 0 & 1.268 & -3.327 & -1.311 & C \\
\hline HETATM & 30 & $\mathrm{H}$ & 0 & 2.124 & -2.852 & -1.792 & $\mathrm{H}$ \\
\hline HETATM & 31 & $\mathrm{H}$ & 0 & 0.449 & -3.388 & -2.035 & $\mathrm{H}$ \\
\hline HETATM & 32 & $\mathrm{H}$ & 0 & 1.549 & -4.344 & -1.016 & $\mathrm{H}$ \\
\hline HETATM & 33 & C & 0 & 2.117 & -2.629 & 1.357 & C \\
\hline HETATM & 34 & $\mathrm{H}$ & 0 & 1.856 & -2.191 & 2.325 & $\mathrm{H}$ \\
\hline HETATM & 35 & $\mathrm{H}$ & 0 & 3.02 & -2.124 & 0.999 & $H$ \\
\hline HETATM & 36 & $\mathrm{H}$ & 0 & 2.321 & -3.698 & 1.488 & $\mathrm{H}$ \\
\hline HETATM & 37 & C & 0 & -0.573 & -3.532 & 0.812 & C \\
\hline HETATM & 38 & $\mathrm{H}$ & 0 & -0.898 & -3.243 & 1.813 & $\mathrm{H}$ \\
\hline HETATM & 39 & $\mathrm{H}$ & 0 & -0.184 & -4.556 & 0.844 & $\mathrm{H}$ \\
\hline HETATM & 40 & $\mathrm{H}$ & 0 & -1.444 & -3.5 & 0.151 & $\mathrm{H}$ \\
\hline HETATM & 41 & $\mathrm{~N}$ & 0 & 1.133 & 0.582 & 1.221 & N \\
\hline HETATM & 42 & 0 & 0 & 1.832 & 0.923 & 2.148 & \\
\hline
\end{tabular}




\begin{tabular}{|c|c|c|c|c|c|c|}
\hline HETATM & 43 & C & 0 & 0.921 & 1.279 & -1.71 \\
\hline HETATM & 44 & C & 0 & 1.475 & -0.059 & -1.733 \\
\hline HETATM & 45 & C & 0 & 1.836 & 2.383 & -1.372 \\
\hline HETATM & 46 & $\mathrm{H}$ & 0 & 1.52 & 3.407 & -1.55 \\
\hline HETATM & 47 & $\mathrm{H}$ & 0 & 0.133 & 1.524 & -2.422 \\
\hline HETATM & 48 & $\mathrm{H}$ & 0 & 1.129 & -0.704 & -2.543 \\
\hline HETATM & 49 & C & 0 & 3.059 & 2.169 & -0.852 \\
\hline HETATM & 50 & $\mathrm{H}$ & 0 & 3.724 & 2.974 & -0.564 \\
\hline HETATM & 51 & C & 0 & 2.989 & -0.157 & -1.59 \\
\hline HETATM & 52 & $\mathrm{H}$ & 0 & 3.444 & -0.001 & -2.584 \\
\hline HETATM & 53 & $N$ & 0 & 3.542 & 0.856 & -0.682 \\
\hline HETATM & 54 & C & 0 & 4.454 & 0.47 & 0.276 \\
\hline HETATM & 55 & $\mathrm{O}$ & 0 & 4.821 & -0.701 & 0.387 \\
\hline HETATM & 56 & C & 0 & 4.982 & 1.554 & 1.199 \\
\hline HETATM & 57 & $\mathrm{H}$ & 0 & 5.503 & 2.345 & 0.647 \\
\hline HETATM & 58 & $\mathrm{H}$ & 0 & 4.158 & 2.007 & 1.759 \\
\hline HETATM & 59 & $\mathrm{H}$ & 0 & 5.683 & 1.086 & 1.892 \\
\hline HETATM & 60 & $\mathrm{H}$ & 0 & 3.339 & -1.12 & -1.223 \\
\hline \multicolumn{7}{|l|}{ END } \\
\hline CONECT & 1 & 2 & 3 & 4 & 5 & \\
\hline CONECT & 2 & 1 & 6 & 9 & & \\
\hline CONECT & 3 & 1 & 14 & 17 & & \\
\hline CONECT & 4 & 1 & 10 & 13 & & \\
\hline CONECT & 5 & 1 & & & & \\
\hline CONECT & 6 & 2 & 7 & & & \\
\hline CONECT & 7 & 6 & 8 & 18 & & \\
\hline CONECT & 8 & 7 & 9 & 19 & & \\
\hline CONECT & 9 & 2 & 8 & 20 & & \\
\hline CONECT & 10 & 4 & 11 & 21 & & \\
\hline CONECT & 11 & 10 & 12 & 22 & & \\
\hline CONECT & 12 & 11 & 13 & 23 & & \\
\hline CONECT & 13 & 4 & 12 & & & \\
\hline CONECT & 14 & 3 & 15 & 24 & & \\
\hline CONECT & 15 & 14 & 16 & 25 & & \\
\hline CONECT & 16 & 15 & 17 & 26 & & \\
\hline CONECT & 17 & 3 & 16 & & & \\
\hline CONECT & 18 & 7 & & & & \\
\hline CONECT & 19 & 8 & & & & \\
\hline CONECT & 20 & 9 & & & & \\
\hline CONECT & 21 & 10 & & & & \\
\hline CONECT & 22 & 11 & & & & \\
\hline CONECT & 23 & 12 & & & & \\
\hline CONECT & 24 & 14 & & & & \\
\hline CONECT & 25 & 15 & & & & \\
\hline
\end{tabular}




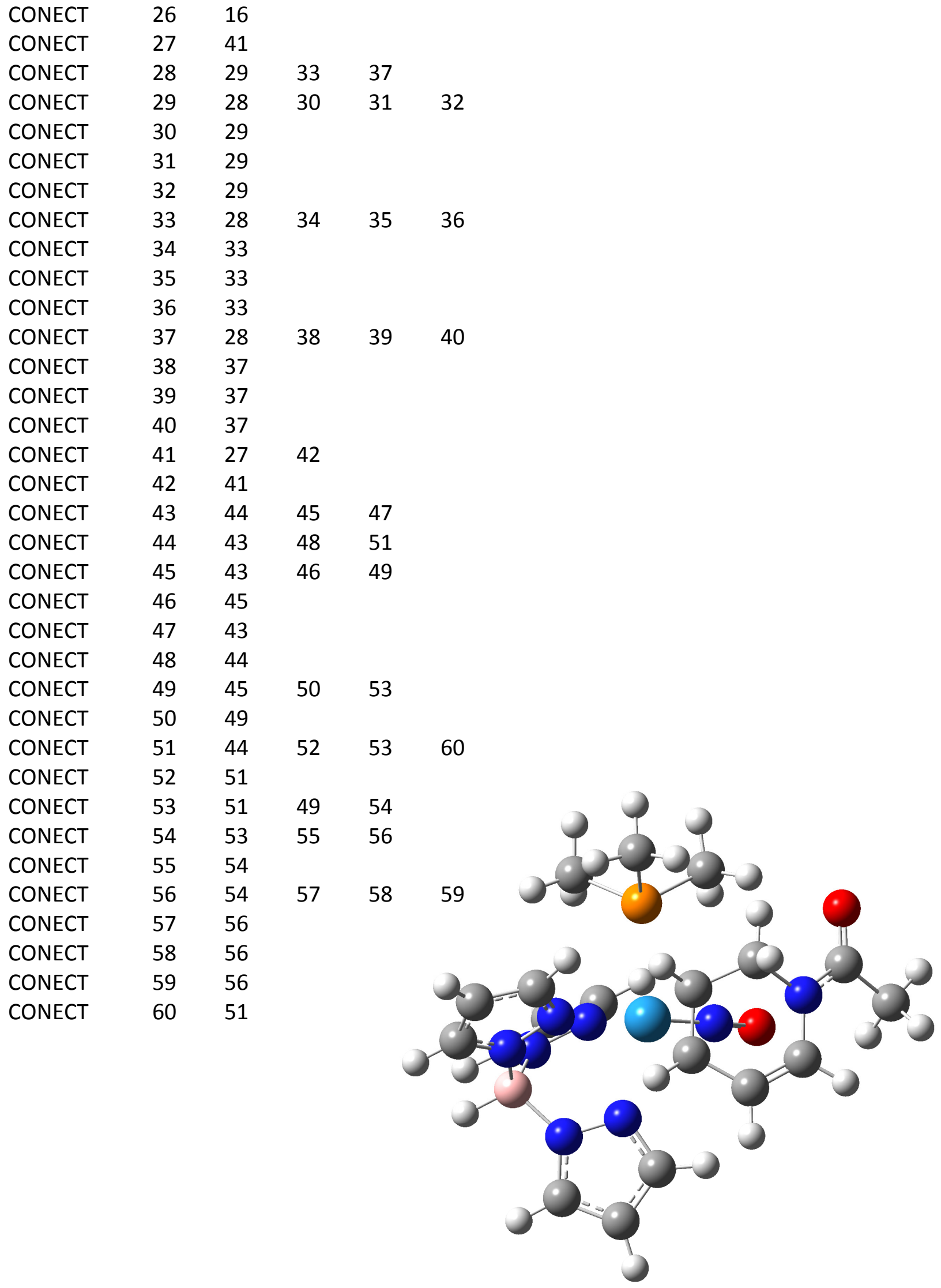


TITLE

REMARK

HETATM

HETATM

HETATM

HETATM

HETATM

HETATM

HETATM

HETATM

HETATM

HETATM

HETATM

HETATM

HETATM

HETATM

HETATM

HETATM

HETATM

HETATM

HETATM 19

HETATM 20

HETATM 21

HETATM 22

HETATM 23

HETATM 24

HETATM $25 \quad \mathrm{H}$

HETATM 26 H 0

$\begin{array}{llll}\text { HETATM } & 27 & W & 0\end{array}$

$\begin{array}{llll}\text { HETATM } & 28 & P & 0\end{array}$

$\begin{array}{llll}\text { HETATM } & 29 & \text { C } & 0\end{array}$

$\begin{array}{llll}\text { HETATM } & 30 & \mathrm{H} & 0\end{array}$

$\begin{array}{llll}\text { HETATM } & 31 & \mathrm{H} & 0\end{array}$

$\begin{array}{llll}\text { HETATM } & 32 & \mathrm{H} & 0\end{array}$

HETATM 33 C 0

$\begin{array}{llll}\text { HETATM } & 34 & \mathrm{H} & 0\end{array}$

$\begin{array}{llll}\text { HETATM } & 35 & \mathrm{H} & 0\end{array}$

HETATM 36 H

$\begin{array}{llll}\text { HETATM } & 37 & \mathrm{C} & 0\end{array}$

$\begin{array}{llll}\text { HETATM } & 38 & \mathrm{H} & 0\end{array}$

$\begin{array}{llll}\text { HETATM } & 39 & \mathrm{H} & 0\end{array}$

HETATM $40 \quad \mathrm{H} \quad 0$

HETATM $41 \quad \mathrm{~N} \quad 0$

$\begin{array}{llll}\text { HETATM } & 42 & 0 & 0\end{array}$ $\begin{array}{llll}2.185 & 1.562 & 0.26 \quad B\end{array}$

$\begin{array}{llll}1.324 & 1.37 & 1.54 & \mathrm{~N}\end{array}$

$\begin{array}{lll}2.766 & 0.163 & -0.058 \mathrm{~N}\end{array}$

$\begin{array}{lll}1.295 & 2.029 & -0.902 \mathrm{~N}\end{array}$

$\begin{array}{lllll}3.061 & 2.351 & 0.459 & \mathrm{H}\end{array}$

$\begin{array}{lllll}0.374 & 0.391 & 1.563 & N\end{array}$

$\begin{array}{lllll}0.072 & 0.165 & 2.856 & \mathrm{C}\end{array}$

$\begin{array}{lllll}0.811 & 1.016 & 3.68 & C\end{array}$

$\begin{array}{llll}1.6 & 1.752 & 2.803 \quad \mathrm{C}\end{array}$

$1.398 \quad 3.141-1.656 \mathrm{C}$

$0.396 \quad 3.118-2.618 \mathrm{C}$

$\begin{array}{lll}-0.29 & 1.926 & -2.378 \mathrm{C}\end{array}$

$\begin{array}{lll}0.254 & 1.265 & -1.337 \mathrm{~N}\end{array}$

$\begin{array}{lllll}4.047 & -0.248 & 0.05 & C\end{array}$

$\begin{array}{llll}4.081 & -1.633 & -0.017 \mathrm{C}\end{array}$

$2.744-2.01 \quad-0.158 \mathrm{C}$

$1.949-0.925-0.194 \mathrm{~N}$

$\begin{array}{llll}-0.643 & -0.599 & 3.123 \quad \mathrm{H}\end{array}$

$\begin{array}{lllll}0.789 & 1.077 & 4.757 \quad \mathrm{H}\end{array}$

$\begin{array}{lllll}2.35 & 2.506 & 2.992 & \mathrm{H}\end{array}$

$2.175 \quad 3.865-1.46 \quad H$

$0.199 \quad 3.853-3.384 \mathrm{H}$

$-1.1361 .516-2.911 \mathrm{H}$

$\begin{array}{llll}4.842 & 0.474 & 0.172 \quad \mathrm{H}\end{array}$

$\begin{array}{lllll}4.946 & -2.277 & 0.033 \quad \mathrm{H}\end{array}$

$2.321-3.002-0.226 \mathrm{H}$

$-0.271-0.652-0.256 \mathrm{~W}$

$\begin{array}{lllll}-2.476 & 0.571 & 0.448 & P\end{array}$

$-3.7611 .045-0.791 \mathrm{C}$

$-4.1950 .166-1.273 \mathrm{H}$

$-3.3341 .699-1.557 \mathrm{H}$

$\begin{array}{llll}-4.56 & 1.591 & -0.279 \mathrm{H}\end{array}$

$-3.463-0.4041 .662 \quad C$

$-2.876-0.6192 .557 \quad \mathrm{H}$

$-3.759-1.3571 .215 \quad H$

$\begin{array}{llll}-4.361 & 0.15 & 1.954 \quad \mathrm{H}\end{array}$

$\begin{array}{llll}-2.196 & 2.2 & 1.272 \quad \mathrm{C}\end{array}$

$\begin{array}{llll}-1.616 & 2.087 & 2.189 \quad \mathrm{H}\end{array}$

$\begin{array}{llll}-3.161 & 2.656 & 1.514 \quad H\end{array}$

$\begin{array}{llll}-1.652 & 2.863 & 0.593 \quad H\end{array}$

$\begin{array}{lllll}-0.771 & -2.054 & 0.742 & N\end{array}$

$\begin{array}{llll}-1.145 & -2.947 & 1.436 & \mathrm{O}\end{array}$ 


\begin{tabular}{|c|c|c|c|c|c|}
\hline HETATM & 43 & $\mathrm{C}$ & 0 & -0.526 & $-1.481-2.468 C$ \\
\hline HETATM & 44 & C & 0 & -1.795 & $-1.085-1.961 C$ \\
\hline HETATM & 45 & $\mathrm{C}$ & 0 & 0.154 & $-2.538-1.891 C$ \\
\hline HETATM & 46 & $\mathrm{H}$ & 0 & 1.183 & $-2.726-2.173 H$ \\
\hline HETATM & 47 & $\mathrm{H}$ & 0 & 0.01 & $-0.827-3.152$ ト \\
\hline HETATM & 48 & $\mathrm{H}$ & 0 & -2.289 & $-0.264-2.466$ ト \\
\hline HETATM & 49 & $\mathrm{H}$ & 0 & -0.374 & $-3.348-1.398$ ト \\
\hline HETATM & 50 & $\mathrm{H}$ & 0 & -2.475 & $-1.864-1.618$ \\
\hline \multicolumn{6}{|l|}{ END } \\
\hline CONECT & 1 & 2 & 3 & 4 & 5 \\
\hline CONECT & 2 & 1 & 6 & 9 & \\
\hline CONECT & 3 & 1 & 14 & 17 & \\
\hline CONECT & 4 & 1 & 10 & 13 & \\
\hline CONECT & 5 & 1 & & & \\
\hline CONECT & 6 & 2 & 7 & & \\
\hline CONECT & 7 & 6 & 8 & 18 & \\
\hline CONECT & 8 & 7 & 9 & 19 & \\
\hline CONECT & 9 & 2 & 8 & 20 & \\
\hline CONECT & 10 & 4 & 11 & 21 & \\
\hline CONECT & 11 & 10 & 12 & 22 & \\
\hline CONECT & 12 & 11 & 13 & 23 & \\
\hline CONECT & 13 & 4 & 12 & & \\
\hline CONECT & 14 & 3 & 15 & 24 & \\
\hline CONECT & 15 & 14 & 16 & 25 & \\
\hline CONECT & 16 & 15 & 17 & 26 & \\
\hline CONECT & 17 & 16 & 3 & & \\
\hline CONECT & 18 & 7 & & & \\
\hline CONECT & 19 & 8 & & & \\
\hline CONECT & 20 & 9 & & & \\
\hline CONECT & 21 & 10 & & & \\
\hline CONECT & 22 & 11 & & & \\
\hline CONECT & 23 & 12 & & & \\
\hline CONECT & 24 & 14 & & & \\
\hline CONECT & 25 & 15 & & & \\
\hline CONECT & 26 & 16 & & & \\
\hline CONECT & 27 & 41 & & & \\
\hline CONECT & 28 & 29 & 33 & 37 & \\
\hline CONECT & 29 & 28 & 30 & 31 & 32 \\
\hline CONECT & 30 & 29 & & & \\
\hline CONECT & 31 & 29 & & & \\
\hline CONECT & 32 & 29 & & & \\
\hline CONECT & 33 & 28 & 34 & 35 & 36 \\
\hline CONECT & 34 & 33 & & & \\
\hline CONECT & 35 & 33 & & & \\
\hline
\end{tabular}




$\begin{array}{llllll}\text { CONECT } & 36 & 33 & & & \\ \text { CONECT } & 37 & 28 & 38 & 39 & 40 \\ \text { CONECT } & 38 & 37 & & & \\ \text { CONECT } & 39 & 37 & & & \\ \text { CONECT } & 40 & 37 & & & \\ \text { CONECT } & 41 & 27 & 42 & & \\ \text { CONECT } & 42 & 41 & & & \\ \text { CONECT } & 43 & 44 & 45 & 47 & \\ \text { CONECT } & 44 & 43 & 48 & 50 & \\ \text { CONECT } & 45 & 43 & 46 & 49 \\ \text { CONECT } & 46 & 45 & & & \\ \text { CONECT } & 47 & 43 & & & \\ \text { CONECT } & 48 & 44 & & \\ \text { CONECT } & 49 & 45 & & \\ \text { CONECT } & 50 & 44 & & \end{array}$
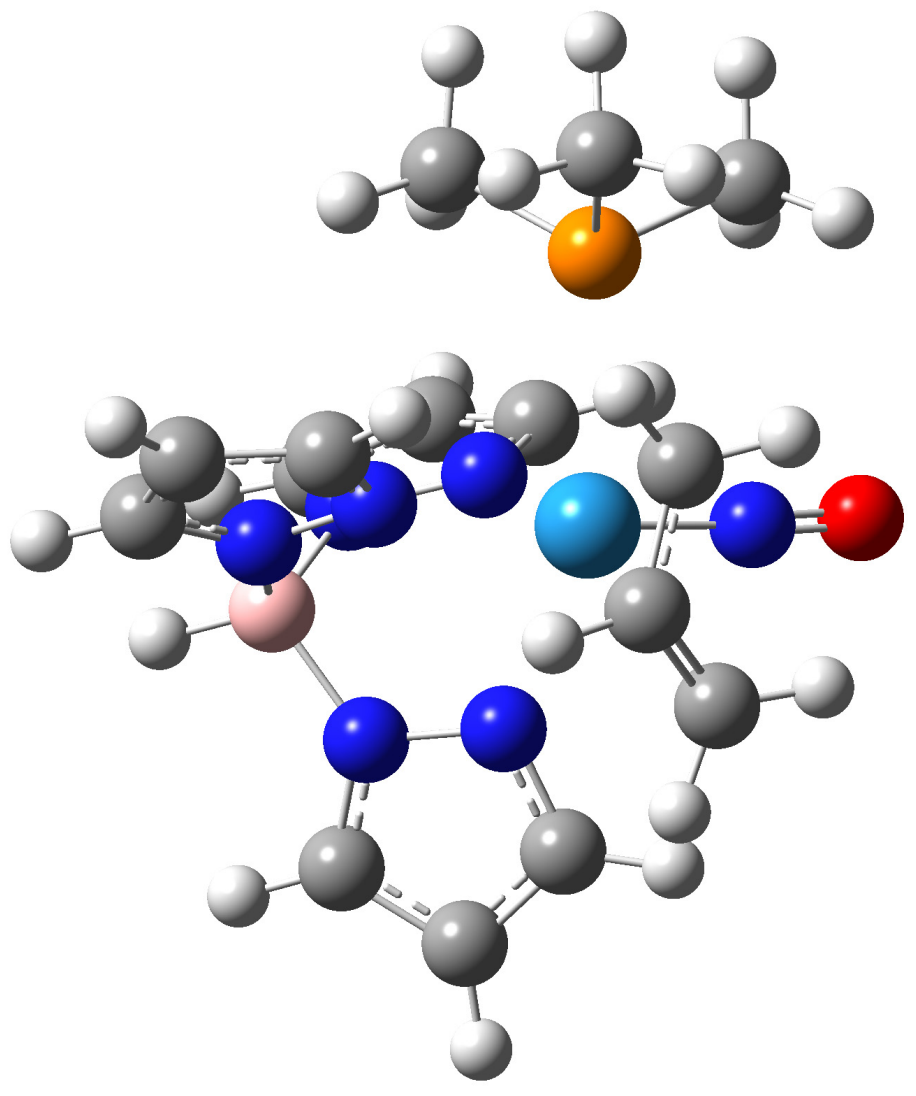
TITLE

REMARK 1 File created by GaussView 5.0.8

\begin{tabular}{|c|c|c|c|c|c|c|}
\hline HETATM & 1 & B & 0 & 2.149 & 1.588 & 0.239 \\
\hline HETATM & 2 & $\mathrm{~N}$ & 0 & 1.295 & 1.41 & 1.525 \\
\hline HETATM & 3 & $\mathrm{~N}$ & 0 & 2.747 & 0.189 & -0.055 \\
\hline HETATM & 4 & $\mathrm{~N}$ & 0 & 1.248 & 2.019 & -0.932 \\
\hline HETATM & 5 & $\mathrm{H}$ & 0 & 3.015 & 2.393 & 0.416 \\
\hline HETATM & 6 & $\mathrm{~N}$ & 0 & 0.365 & 0.414 & 1.567 \\
\hline HETATM & 7 & C & 0 & 0.084 & 0.188 & 2.863 \\
\hline HETATM & 8 & C & 0 & 0.816 & 1.06 & 3.673 \\
\hline HETATM & 9 & $C$ & 0 & 1.581 & 1.804 & 2.784 \\
\hline HETATM & 10 & C & 0 & 1.359 & 3.108 & -1.721 \\
\hline HETATM & 11 & C & 0 & 0.366 & 3.058 & -2.69 \\
\hline HETATM & 12 & C & 0 & -0.325 & 1.874 & -2.419 \\
\hline HETATM & 13 & $\mathrm{~N}$ & 0 & 0.207 & 1.244 & -1.353 \\
\hline HETATM & 14 & $C$ & 0 & 4.032 & -0.217 & 0.022 \\
\hline HETATM & 15 & C & 0 & 4.069 & -1.603 & -0.044 \\
\hline HETATM & 16 & C & 0 & 2.729 & -1.986 & -0.152 \\
\hline HETATM & 17 & $N$ & 0 & 1.935 & -0.902 & -0.164 \\
\hline HETATM & 18 & $\mathrm{H}$ & 0 & -0.615 & -0.587 & 3.143 \\
\hline HETATM & 19 & $\mathrm{H}$ & 0 & 0.805 & 1.128 & 4.751 \\
\hline HETATM & 20 & $\mathrm{H}$ & 0 & 2.321 & 2.572 & 2.959 \\
\hline HETATM & 21 & $\mathrm{H}$ & 0 & 2.136 & 3.836 & -1.54 \\
\hline HETATM & 22 & $\mathrm{H}$ & 0 & 0.176 & 3.77 & -3.478 \\
\hline HETATM & 23 & $\mathrm{H}$ & 0 & -1.166 & 1.451 & -2.948 \\
\hline HETATM & 24 & $\mathrm{H}$ & 0 & 4.827 & 0.507 & 0.128 \\
\hline HETATM & 25 & $\mathrm{H}$ & 0 & 4.938 & -2.243 & -0.012 \\
\hline HETATM & 26 & $\mathrm{H}$ & 0 & 2.302 & -2.977 & -0.219 \\
\hline HETATM & 27 & W & 0 & -0.277 & -0.666 & -0.24 \\
\hline HETATM & 28 & $P$ & 0 & -2.462 & 0.589 & 0.456 \\
\hline HETATM & 29 & C & 0 & -3.66 & 1.142 & -0.838 \\
\hline HETATM & 30 & $\mathrm{H}$ & 0 & -4.094 & 0.299 & -1.378 \\
\hline HETATM & 31 & $\mathrm{H}$ & 0 & -3.165 & 1.807 & -1.551 \\
\hline HETATM & 32 & $\mathrm{H}$ & 0 & -4.469 & 1.697 & -0.353 \\
\hline HETATM & 33 & $C$ & 0 & -3.52 & -0.399 & 1.596 \\
\hline HETATM & 34 & $\mathrm{H}$ & 0 & -2.964 & -0.659 & 2.5 \\
\hline HETATM & 35 & $\mathrm{H}$ & 0 & -3.826 & -1.328 & 1.107 \\
\hline HETATM & 36 & $\mathrm{H}$ & 0 & -4.414 & 0.167 & 1.881 \\
\hline HETATM & 37 & C & 0 & -2.191 & 2.186 & 1.342 \\
\hline HETATM & 38 & $\mathrm{H}$ & 0 & -1.669 & 2.036 & 2.288 \\
\hline HETATM & 39 & $\mathrm{H}$ & 0 & -3.158 & 2.659 & 1.54 \\
\hline HETATM & 40 & $\mathrm{H}$ & 0 & -1.593 & 2.853 & 0.713 \\
\hline HETATM & 41 & $\mathrm{~N}$ & 0 & -0.62 & -2.106 & 0.77 \\
\hline HETATM & 42 & $\mathrm{O}$ & 0 & -0.842 & -3.038 & 1.477 \\
\hline
\end{tabular}




\begin{tabular}{|c|c|c|c|c|c|c|}
\hline HETATM & 43 & $\mathrm{C}$ & 0 & -0.858 & -2.2 & -2.007 \\
\hline HETATM & 44 & C & 0 & 0.397 & -1.746 & -2.369 \\
\hline HETATM & 45 & $C$ & 0 & -1.899 & -1.248 & -1.805 \\
\hline HETATM & 46 & $\mathrm{H}$ & 0 & -2.851 & -1.639 & -1.455 \\
\hline HETATM & 47 & $\mathrm{H}$ & 0 & -0.977 & -3.218 & -1.642 \\
\hline HETATM & 48 & $\mathrm{H}$ & 0 & 1.247 & -2.418 & -2.377 \\
\hline HETATM & 49 & $\mathrm{H}$ & 0 & -1.998 & -0.453 & -2.54 \\
\hline HETATM & 50 & $\mathrm{H}$ & 0 & 0.519 & -0.821 & -2.923 \\
\hline \multicolumn{7}{|l|}{ END } \\
\hline CONECT & 1 & 2 & 3 & 4 & 5 & \\
\hline CONECT & 2 & 1 & 6 & 9 & & \\
\hline CONECT & 3 & 1 & 14 & 17 & & \\
\hline CONECT & 4 & 1 & 10 & 13 & & \\
\hline CONECT & 5 & 1 & & & & \\
\hline CONECT & 6 & 2 & 7 & & & \\
\hline CONECT & 7 & 6 & 8 & 18 & & \\
\hline CONECT & 8 & 7 & 9 & 19 & & \\
\hline CONECT & 9 & 2 & 8 & 20 & & \\
\hline CONECT & 10 & 4 & 11 & 21 & & \\
\hline CONECT & 11 & 10 & 12 & 22 & & \\
\hline CONECT & 12 & 11 & 13 & 23 & & \\
\hline CONECT & 13 & 4 & 12 & & & \\
\hline CONECT & 14 & 3 & 15 & 24 & & \\
\hline CONECT & 15 & 14 & 16 & 25 & & \\
\hline CONECT & 16 & 15 & 17 & 26 & & \\
\hline CONECT & 17 & 16 & 3 & & & \\
\hline CONECT & 18 & 7 & & & & \\
\hline CONECT & 19 & 8 & & & & \\
\hline CONECT & 20 & 9 & & & & \\
\hline CONECT & 21 & 10 & & & & \\
\hline CONECT & 22 & 11 & & & & \\
\hline CONECT & 23 & 12 & & & & \\
\hline CONECT & 24 & 14 & & & & \\
\hline CONECT & 25 & 15 & & & & \\
\hline CONECT & 26 & 16 & & & & \\
\hline CONECT & 27 & 41 & & & & \\
\hline CONECT & 28 & 29 & 33 & 37 & & \\
\hline CONECT & 29 & 28 & 30 & 31 & 32 & \\
\hline CONECT & 30 & 29 & & & & \\
\hline CONECT & 31 & 29 & & & & \\
\hline CONECT & 32 & 29 & & & & \\
\hline CONECT & 33 & 28 & 34 & 35 & 36 & \\
\hline CONECT & 34 & 33 & & & & \\
\hline CONECT & 35 & 33 & & & & \\
\hline
\end{tabular}




$\begin{array}{llllll}\text { CONECT } & 36 & 33 & & & \\ \text { CONECT } & 37 & 28 & 38 & 39 & 40 \\ \text { CONECT } & 38 & 37 & & & \\ \text { CONECT } & 39 & 37 & & & \\ \text { CONECT } & 40 & 37 & & & \\ \text { CONECT } & 41 & 27 & 42 & & \\ \text { CONECT } & 42 & 41 & & & \\ \text { CONECT } & 43 & 44 & 45 & 47 & \\ \text { CONECT } & 44 & 43 & 48 & 50 & \\ \text { CONECT } & 45 & 43 & 46 & 49 \\ \text { CONECT } & 46 & 45 & & & \\ \text { CONECT } & 47 & 43 & & & \\ \text { CONECT } & 48 & 44 & & & \\ \text { CONECT } & 49 & 45 & & \\ \text { CONECT } & 50 & 44 & & \end{array}$

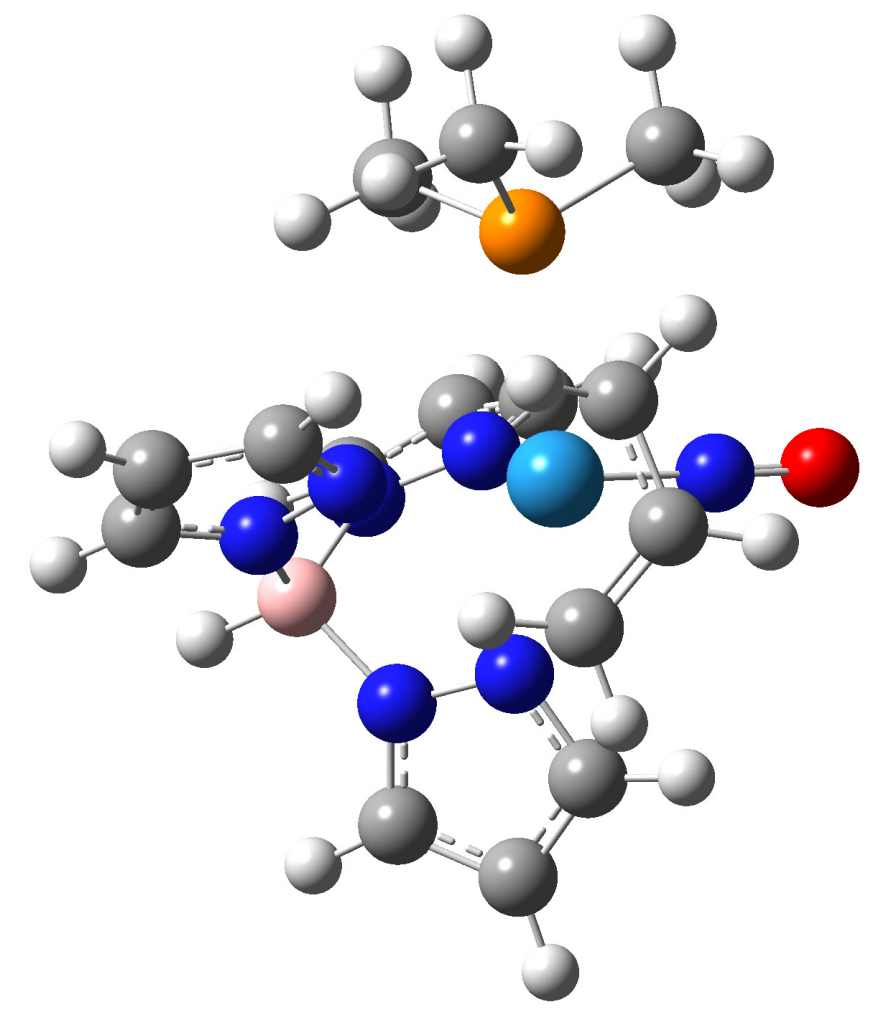




\begin{tabular}{|c|c|c|c|c|c|c|c|}
\hline TITLE & $11 k$ & & & & & & \\
\hline REMARK & 1 & & eat & y Gauss & View 5. & 0.8 & \\
\hline HETATM & 1 & $B$ & 0 & 2.634 & -1.04 & 0.143 & B \\
\hline HETATM & 2 & $\mathrm{~N}$ & 0 & 1.824 & -1.549 & -1.082 & $\mathrm{~N}$ \\
\hline HETATM & 3 & $\mathrm{~N}$ & 0 & 2.77 & 0.485 & -0.04 & \\
\hline HETATM & 4 & $\mathrm{~N}$ & 0 & 1.827 & -1.326 & 1.431 & $\mathrm{~N}$ \\
\hline HETATM & 5 & $\mathrm{H}$ & 0 & 3.704 & -1.571 & 0.193 & $\mathrm{H}$ \\
\hline HETATM & 6 & $\mathrm{~N}$ & 0 & 0.637 & -0.953 & -1.392 & $\mathrm{~N}$ \\
\hline HETATM & 7 & C & 0 & 0.281 & -1.392 & -2.619 & C \\
\hline HETATM & 8 & C & 0 & 1.228 & -2.298 & -3.094 & C \\
\hline HETATM & 9 & C & 0 & 2.194 & -2.356 & -2.092 & C \\
\hline HETATM & 10 & C & 0 & 2.201 & -1.99 & 2.542 & C \\
\hline HETATM & 11 & C & 0 & 1.167 & -1.93 & 3.469 & C \\
\hline HETATM & 12 & C & 0 & 0.17 & -1.19 & 2.829 & C \\
\hline HETATM & 13 & $\mathrm{~N}$ & 0 & 0.569 & -0.825 & 1.595 & $\mathrm{~N}$ \\
\hline HETATM & 14 & C & 0 & 3.872 & 1.25 & -0.196 & C \\
\hline HETATM & 15 & C & 0 & 3.475 & 2.552 & -0.469 & C \\
\hline HETATM & 16 & C & 0 & 2.077 & 2.505 & -0.471 & C \\
\hline HETATM & 17 & $\mathrm{~N}$ & 0 & 1.658 & 1.257 & -0.209 & $\mathrm{~N}$ \\
\hline HETATM & 18 & $\mathrm{H}$ & 0 & -0.615 & -1.016 & -3.09 & $\mathrm{H}$ \\
\hline HETATM & 19 & $\mathrm{H}$ & 0 & 1.226 & -2.824 & -4.037 & $\mathrm{H}$ \\
\hline HETATM & 20 & $\mathrm{H}$ & 0 & 3.122 & -2.907 & -2.04 & $\mathrm{H}$ \\
\hline HETATM & 21 & $\mathrm{H}$ & 0 & 3.174 & -2.456 & 2.599 & $\mathrm{H}$ \\
\hline HETATM & 22 & $\mathrm{H}$ & 0 & 1.144 & -2.357 & 4.46 & $\mathrm{H}$ \\
\hline HETATM & 23 & $\mathrm{H}$ & 0 & -0.802 & -0.896 & 3.2 & $\mathrm{H}$ \\
\hline HETATM & 24 & $\mathrm{H}$ & 0 & 4.856 & 0.814 & -0.105 & $\mathrm{H}$ \\
\hline HETATM & 25 & $\mathrm{H}$ & 0 & 4.107 & 3.409 & -0.647 & $\mathrm{H}$ \\
\hline HETATM & 26 & $\mathrm{H}$ & 0 & 1.358 & 3.293 & -0.644 & $\mathrm{H}$ \\
\hline HETATM & 27 & W & 0 & -0.382 & 0.389 & -0.026 & n \\
\hline HETATM & 28 & $P$ & 0 & -2.461 & -1.16 & 0.039 & $P$ \\
\hline HETATM & 29 & C & 0 & -3.718 & -0.745 & 1.325 & C \\
\hline HETATM & 30 & $\mathrm{H}$ & 0 & -4.1 & 0.268 & 1.161 & $\mathrm{H}$ \\
\hline HETATM & 31 & $\mathrm{H}$ & 0 & -3.277 & -0.795 & 2.325 & $\mathrm{H}$ \\
\hline HETATM & 32 & $\mathrm{H}$ & 0 & -4.555 & -1.449 & 1.274 & $\mathrm{H}$ \\
\hline HETATM & 33 & C & 0 & -3.433 & -1.165 & -1.525 & C \\
\hline HETATM & 34 & $\mathrm{H}$ & 0 & -2.843 & -1.618 & -2.327 & $\mathrm{H}$ \\
\hline HETATM & 35 & $\mathrm{H}$ & 0 & -3.679 & -0.14 & -1.817 & $\mathrm{H}$ \\
\hline HETATM & 36 & $\mathrm{H}$ & 0 & -4.356 & -1.74 & -1.401 & $\mathrm{H}$ \\
\hline HETATM & 37 & C & 0 & -2.073 & -2.935 & 0.34 & C \\
\hline HETATM & 38 & $\mathrm{H}$ & 0 & -1.392 & -3.296 & -0.435 & $\mathrm{H}$ \\
\hline HETATM & 39 & $\mathrm{H}$ & 0 & -2.988 & -3.536 & 0.323 & $\mathrm{H}$ \\
\hline HETATM & 40 & $\mathrm{H}$ & 0 & -1.582 & -3.051 & 1.31 & $\mathrm{H}$ \\
\hline HETATM & 41 & $\mathrm{~N}$ & 0 & -1.155 & 1.318 & -1.353 & $\mathrm{~N}$ \\
\hline HETATM & 42 & 0 & 0 & -1.683 & 1.904 & -2.244 & \\
\hline
\end{tabular}




\begin{tabular}{|c|c|c|c|c|c|c|}
\hline HETATM & 43 & C & 0 & -0.961 & 3.352 & 1.209 \\
\hline HETATM & 44 & C & 0 & -0.578 & 1.943 & 1.38 \\
\hline HETATM & 45 & C & 0 & -2.083 & 3.806 & 0.626 \\
\hline HETATM & 46 & $\mathrm{H}$ & 0 & 0.048 & 1.784 & 2.262 \\
\hline HETATM & 47 & $\mathrm{H}$ & 0 & -1.513 & 1.266 & 1.464 \\
\hline HETATM & 48 & $\mathrm{H}$ & 0 & -2.844 & 3.135 & 0.235 \\
\hline HETATM & 49 & $\mathrm{H}$ & 0 & -2.282 & 4.869 & 0.537 \\
\hline HETATM & 50 & $\mathrm{H}$ & 0 & -0.254 & 4.075 & 1.616 \\
\hline \multicolumn{7}{|l|}{ END } \\
\hline CONECT & 1 & 2 & 3 & 4 & 5 & \\
\hline CONECT & 2 & 1 & 6 & 9 & & \\
\hline CONECT & 3 & 1 & 14 & 17 & & \\
\hline CONECT & 4 & 1 & 10 & 13 & & \\
\hline CONECT & 5 & 1 & & & & \\
\hline CONECT & 6 & 2 & 7 & & & \\
\hline CONECT & 7 & 6 & 8 & 18 & & \\
\hline CONECT & 8 & 7 & 9 & 19 & & \\
\hline CONECT & 9 & 2 & 8 & 20 & & \\
\hline CONECT & 10 & 4 & 11 & 21 & & \\
\hline CONECT & 11 & 10 & 12 & 22 & & \\
\hline CONECT & 12 & 11 & 13 & 23 & & \\
\hline CONECT & 13 & 4 & 12 & & & \\
\hline CONECT & 14 & 3 & 15 & 24 & & \\
\hline CONECT & 15 & 14 & 16 & 25 & & \\
\hline CONECT & 16 & 15 & 17 & 26 & & \\
\hline CONECT & 17 & 3 & 16 & & & \\
\hline CONECT & 18 & 7 & & & & \\
\hline CONECT & 19 & 8 & & & & \\
\hline CONECT & 20 & 9 & & & & \\
\hline CONECT & 21 & 10 & & & & \\
\hline CONECT & 22 & 11 & & & & \\
\hline CONECT & 23 & 12 & & & & \\
\hline CONECT & 24 & 14 & & & & \\
\hline CONECT & 25 & 15 & & & & \\
\hline CONECT & 26 & 16 & & & & \\
\hline CONECT & 27 & 41 & 44 & & & \\
\hline CONECT & 28 & 29 & 33 & 37 & & \\
\hline CONECT & 29 & 28 & 30 & 31 & 32 & \\
\hline CONECT & 30 & 29 & & & & \\
\hline CONECT & 31 & 29 & & & & \\
\hline CONECT & 32 & 29 & & & & \\
\hline CONECT & 33 & 28 & 34 & 35 & 36 & \\
\hline CONECT & 34 & 33 & & & & \\
\hline CONECT & 35 & 33 & & & & \\
\hline
\end{tabular}




$\begin{array}{llllll}\text { CONECT } & 36 & 33 & & & \\ \text { CONECT } & 37 & 28 & 38 & 39 & 40 \\ \text { CONECT } & 38 & 37 & & & \\ \text { CONECT } & 39 & 37 & & & \\ \text { CONECT } & 40 & 37 & & & \\ \text { CONECT } & 41 & 27 & 42 & & \\ \text { CONECT } & 42 & 41 & & & \\ \text { CONECT } & 43 & 44 & 45 & 50 & \\ \text { CONECT } & 44 & 27 & 43 & 46 \\ \text { CONECT } & 45 & 43 & 48 & 49 \\ \text { CONECT } & 46 & 44 & & & \\ \text { CONECT } & 48 & 45 & & & \\ \text { CONECT } & 49 & 45 & & \\ \text { CONECT } & 50 & 43 & & \end{array}$
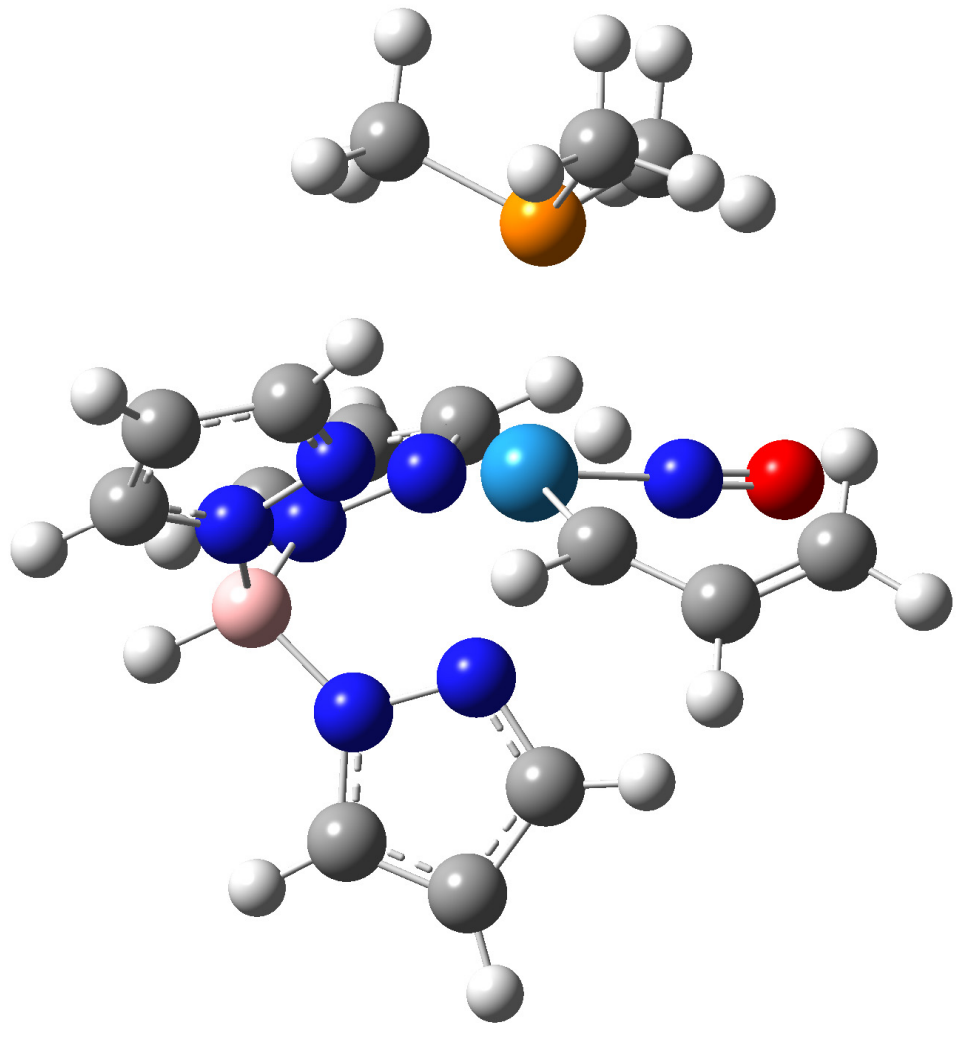
TITLE

HETATM

HETATM 2

HETATM 3

HETATM 4

HETATM

HETATM

HETATM

HETATM

HETATM

HETATM 10

HETATM 11

HETATM 12

HETATM 13

HETATM 14

HETATM 15

HETATM 16

HETATM 17

HETATM 18

HETATM 19

HETATM 20

HETATM 21

HETATM 22

HETATM 23

HETATM $24 \quad$

HETATM 25

HETATM 26

HETATM $27 \quad W \quad$

$\begin{array}{llll}\text { HETATM } & 28 & P & 0\end{array}$

$\begin{array}{llll}\text { HETATM } & 29 & \text { C } & 0\end{array}$

$\begin{array}{llll}\text { HETATM } & 30 & \mathrm{H} & 0\end{array}$

$\begin{array}{llll}\text { HETATM } & 31 & \mathrm{H} & 0\end{array}$

$\begin{array}{llll}\text { HETATM } & 32 & \mathrm{H} & 0\end{array}$

HETATM 33 C 0

$\begin{array}{llll}\text { HETATM } & 34 & \mathrm{H} & 0\end{array}$

HETATM 35 H 0

HETATM $36 \quad \mathrm{H} \quad 0$

$\begin{array}{llll}\text { HETATM } & 37 & \mathrm{C} & 0\end{array}$

$\begin{array}{llll}\text { HETATM } & 38 & \mathrm{H} & 0\end{array}$

HETATM $39 \quad \mathrm{H} \quad 0$

$\begin{array}{llll}\text { HETATM } & 40 & \mathrm{H} & 0\end{array}$

HETATM 41 N 0

$\begin{array}{llll}\text { HETATM } & 42 & 0 & 0\end{array}$ $\begin{array}{llll}0.711 & 2.758 & 0.396 & B\end{array}$

$\begin{array}{lll}1.562 & 2.122 & -0.738 \mathrm{~N}\end{array}$

$\begin{array}{lll}-0.762 & 2.667 & -0.074 N\end{array}$

$\begin{array}{llll}0.912 & 1.946 & 1.682 & N\end{array}$

$\begin{array}{lllll}1.027 & 3.899 & 0.567 \quad \mathrm{H}\end{array}$

$\begin{array}{llll}1.272 & 0.863 & -1.18 \quad \mathrm{~N}\end{array}$

$\begin{array}{lllll}1.903 & 0.707 & -2.359 \mathrm{C}\end{array}$

$\begin{array}{llll}2.628 & 1.857 & -2.677 \quad \mathrm{C}\end{array}$

$\begin{array}{llll}2.372 & 2.732 & -1.628 \mathrm{C}\end{array}$

$\begin{array}{lllll}1.457 & 2.337 & 2.851 & \mathrm{C}\end{array}$

$\begin{array}{lllll}1.544 & 1.233 & 3.689 & C\end{array}$

$\begin{array}{llll}1.02 & 0.182 & 2.933 \quad \mathrm{C}\end{array}$

$\begin{array}{llll}0.633 & 0.612 & 1.716 \quad \mathrm{~N}\end{array}$

$\begin{array}{llll}-1.558 & 3.674 & -0.492 C\end{array}$

$-2.6413 .138-1.175 \mathrm{C}$

$-2.4241 .758-1.146 \mathrm{C}$

$\begin{array}{lll}-1.298 & 1.472 & -0.469 \mathrm{~N}\end{array}$

$\begin{array}{llll}1.784 & -0.208 & -2.92 \quad \mathrm{H}\end{array}$

$\begin{array}{llll}3.236 & 2.035 & -3.551 \mathrm{H}\end{array}$

$\begin{array}{llll}2.696 & 3.749 & -1.461 \mathrm{H}\end{array}$

$\begin{array}{lllll}1.744 & 3.367 & 3.004 \quad \mathrm{H}\end{array}$

$1.9251 .198 \quad 4.698 \quad \mathrm{H}$

$\begin{array}{lllll}0.898 & -0.853 & 3.215 \quad \mathrm{H}\end{array}$

$\begin{array}{lll}-1.29 & 4.7 & -0.285 \mathrm{H}\end{array}$

$\begin{array}{lll}-3.461 & 3.67 & -1.634 \mathrm{H}\end{array}$

$-3.0120 .966-1.584 \mathrm{H}$

$-0.198-0.418-0.142 \mathrm{~W}$

$\begin{array}{llll}1.931 & -1.914 & -0.004 P\end{array}$

$\begin{array}{lllll}2.062 & -3.195 & 1.321 \quad C\end{array}$

$\begin{array}{lllll}1.242 & -3.914 & 1.254 \quad \mathrm{H}\end{array}$

$\begin{array}{llll}2.042 & -2.733 & 2.312 \quad \mathrm{H}\end{array}$

$\begin{array}{llll}3.01 & -3.732 & 1.21 \quad \mathrm{H}\end{array}$

$2.205-2.928-1.522 \mathrm{C}$

$2.373-2.289-2.391 \mathrm{H}$

$1.324-3.544-1.722 \mathrm{H}$

$\begin{array}{lllll}3.077 & -3.577 & -1.39 \quad \mathrm{H}\end{array}$

$\begin{array}{lllll}3.52 & -1.003 & 0.226 \quad C\end{array}$

$\begin{array}{lllll}3.692 & -0.309 & -0.6 \quad H\end{array}$

$\begin{array}{lllll}4.352 & -1.712 & 0.277 \quad H\end{array}$

$3.481-0.4321 .158 \quad \mathrm{H}$

$-0.609-1.193-1.697 \mathrm{~N}$

$-0.793-1.696-2.7670$ 


$\begin{array}{llllllll}\text { HETATM } & 43 & \mathrm{C} & 0 & -2.068 & -1.244 & 1.211 & \mathrm{C} \\ \text { HETATM } & 44 & \mathrm{C} & 0 & -0.847 & -2.057 & 1.195 & \mathrm{C} \\ \text { HETATM } & 45 & \mathrm{C} & 0 & -3.029 & -1.366 & 0.21 & \mathrm{C} \\ \text { HETATM } & 46 & \mathrm{H} & 0 & -0.369 & -2.134 & 2.168 & \mathrm{H} \\ \text { HETATM } & 47 & \mathrm{H} & 0 & -0.915 & -3.035 & 0.721 & \mathrm{H} \\ \text { HETATM } & 48 & \mathrm{C} & 0 & -2.376 & -0.373 & 2.424 & \mathrm{C} \\ \text { HETATM } & 49 & \mathrm{H} & 0 & -2.539 & 0.68 & 2.172 & \mathrm{H} \\ \text { HETATM } & 50 & \mathrm{H} & 0 & -3.284 & -0.737 & 2.918 & \mathrm{H} \\ \text { HETATM } & 51 & \mathrm{H} & 0 & -1.568 & -0.413 & 3.154 & \mathrm{H} \\ \text { HETATM } & 52 & \mathrm{C} & 0 & -3.088 & -2.473 & -0.805 & \mathrm{C} \\ \text { HETATM } & 53 & \mathrm{H} & 0 & -3.153 & -2.088 & -1.829 & \mathrm{H} \\ \text { HETATM } & 54 & \mathrm{H} & 0 & -2.252 & -3.169 & -0.763 & \mathrm{H} \\ \text { HETATM } & 55 & \mathrm{H} & 0 & -4.016 & -3.035 & -0.627 & \mathrm{H} \\ \text { HETATM } & 56 & \mathrm{C} & 0 & -4.286 & -0.537 & 0.263 & \mathrm{C} \\ \text { HETATM } & 57 & \mathrm{H} & 0 & -4.992 & -1.017 & 0.959 & \mathrm{H} \\ \text { HETATM } & 58 & \mathrm{H} & 0 & -4.125 & 0.48 & 0.624 & \mathrm{H} \\ \text { HETATM } & 59 & \mathrm{H} & 0 & -4.784 & -0.497 & -0.711 & \mathrm{H} \\ \text { END } & & & & & & & \\ \text { CONECT } & 1 & 2 & 3 & 4 & 5 & & \\ \text { CONECT } & 2 & 1 & 6 & 9 & & & \\ \text { CONECT } & 3 & 1 & 14 & 17 & & & \\ \text { CONECT } & 4 & 1 & 10 & 13 & & & \\ \text { CONECT } & 5 & 1 & & & & & \\ \text { CONECT } & 6 & 2 & 7 & & & & \\ \text { CONECT } & 7 & 6 & 8 & 18 & & & \\ \text { CONECT } & 8 & 7 & 9 & 19 & & & \\ \text { CONECT } & 9 & 2 & 8 & 20 & & & \\ \text { CONECT } & 10 & 4 & 11 & 21 & & & \\ \text { CONECT } & 11 & 10 & 12 & 22 & & & \\ \text { CONECT } & 12 & 11 & 13 & 23 & & & \\ \text { CONECT } & 13 & 4 & 12 & & & & \\ \text { CONECT } & 14 & 3 & 15 & 24 & & & \\ \text { CONECT } & 15 & 14 & 16 & 25 & & & \\ \text { CONECT } & 16 & 15 & 17 & 26 & & & \\ \text { CONECT } & 17 & 16 & 3 & & & & \\ \text { CONECT } & 18 & 7 & & & & & \\ \text { CONECT } & 19 & 8 & & & & & \\ \text { CONECT } & 20 & 9 & & & & & \\ \text { CONECT } & 21 & 10 & & & & & \\ \text { CONECT } & 22 & 11 & & & & & \\ \text { CONECT } & 23 & 12 & & & & & \\ \text { CONECT } & 24 & 14 & & & & & \\ \text { CONECT } & 25 & 15 & & & & & \\ \text { CONECT } & 26 & 16 & & & & & \end{array}$




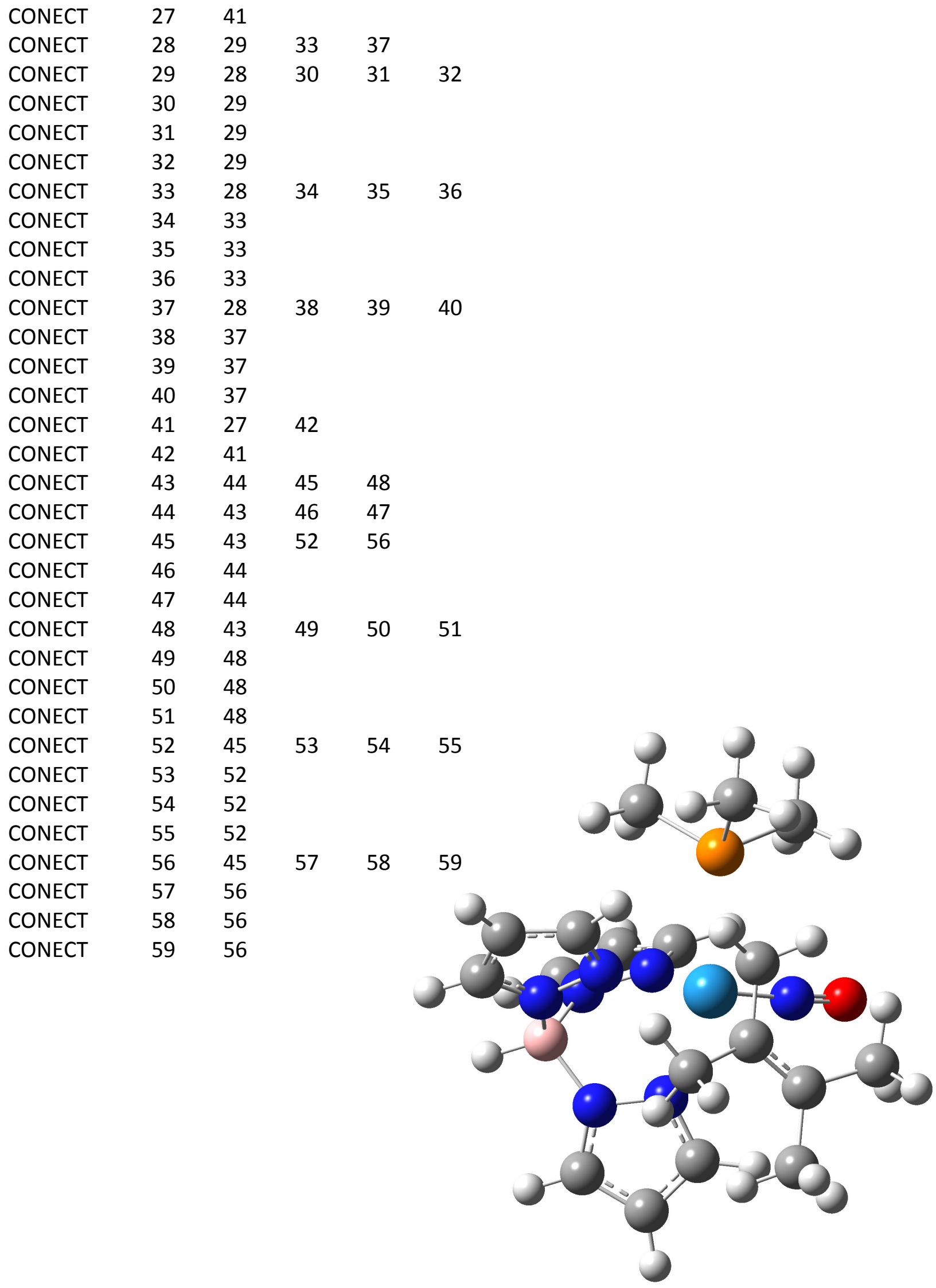


TITLE

REMARK 1 File created by GaussView 5.0.8

$\begin{array}{llllllll}\text { HETATM } & 1 & \text { B } & 0 & -0.54 & 2.721 & 0.558 & \text { B }\end{array}$

$\begin{array}{llllllll}\text { HETATM } & 2 & \mathrm{~N} & 0 & 0.613 & 2.599 & -0.477 \mathrm{~N}\end{array}$

$\begin{array}{lllllll}\text { HETATM } & 3 & \mathrm{~N} & 0 & -1.782 & 2.086 & -0.117 \mathrm{~N}\end{array}$

$\begin{array}{llllllll}\text { HETATM } & 4 & \mathrm{~N} & 0 & -0.163 & 1.937 & 1.824 & \mathrm{~N}\end{array}$

$\begin{array}{llllllllll}\text { HETATM } & 5 & \mathrm{H} & 0 & -0.747 & 3.869 & 0.824 & \mathrm{H}\end{array}$

$\begin{array}{llllllll}\text { HETATM } & 6 & \mathrm{~N} & 0 & 0.911 & 1.379 & -1.012 & \mathrm{~N}\end{array}$

$\begin{array}{llllllll}\text { HETATM } & 7 & \mathrm{C} & 0 & 1.646 & 1.603 & -2.117 \mathrm{C}\end{array}$

$\begin{array}{llllllll}\text { HETATM } & 8 & \mathrm{C} & 0 & 1.85 & 2.974 & -2.292 & \mathrm{C}\end{array}$

$\begin{array}{llllllll}\text { HETATM } & 9 & \mathrm{C} & 0 & 1.166 & 3.566 & -1.238 \mathrm{C}\end{array}$

$\begin{array}{llllllll}\text { HETATM } & 10 & \mathrm{C} & 0 & 0.037 & 2.402 & 3.074 & \mathrm{C}\end{array}$

$\begin{array}{llllllll}\text { HETATM } & 11 & \mathrm{C} & 0 & 0.469 & 1.351 & 3.873 & \mathrm{C}\end{array}$

$\begin{array}{llllllll}\text { HETATM } & 12 & \mathrm{C} & 0 & 0.509 & 0.253 & 3.01 & \mathrm{C}\end{array}$

$\begin{array}{llllllll}\text { HETATM } & 13 & \mathrm{~N} & 0 & 0.124 & 0.605 & 1.768 & \mathrm{~N}\end{array}$

$\begin{array}{llllllll}\text { HETATM } & 14 & \mathrm{C} & 0 & -2.869 & 2.718 & -0.612 & \mathrm{C}\end{array}$

$\begin{array}{llllllll}\text { HETATM } & 15 & \mathrm{C} & 0 & -3.54 & 1.857 & -1.469 & \mathrm{C}\end{array}$

$\begin{array}{llllllll}\text { HETATM } & 16 & \mathrm{C} & 0 & -2.773 & 0.689 & -1.464 \mathrm{C}\end{array}$

$\begin{array}{llllllll}\text { HETATM } & 17 & \mathrm{~N} & 0 & -1.721 & 0.822 & -0.638 \mathrm{~N}\end{array}$

$\begin{array}{lllllll}\text { HETATM } & 18 & \mathrm{H} & 0 & 1.974 & 0.775 & -2.728 \mathrm{H}\end{array}$

$\begin{array}{llllllll}\text { HETATM } & 19 & \mathrm{H} & 0 & 2.402 & 3.464 & -3.08 & \mathrm{H}\end{array}$

$\begin{array}{llllllll}\text { HETATM } & 20 & \mathrm{H} & 0 & 1.021 & 4.607 & -0.987 \mathrm{H}\end{array}$

$\begin{array}{llllllll}\text { HETATM } & 21 & \mathrm{H} & 0 & -0.14 & 3.442 & 3.306 & \mathrm{H}\end{array}$

$\begin{array}{llllllll}\text { HETATM } & 22 & \mathrm{H} & 0 & 0.712 & 1.377 & 4.924 & \mathrm{H}\end{array}$

$\begin{array}{lllllllll}\text { HETATM } & 23 & \mathrm{H} & 0 & 0.78 & -0.768 & 3.234 & \mathrm{H}\end{array}$

$\begin{array}{llllllll}\text { HETATM } & 24 & \mathrm{H} & 0 & -3.08 & 3.739 & -0.328 \mathrm{H}\end{array}$

$\begin{array}{llllllll}\text { HETATM } & 25 & \mathrm{H} & 0 & -4.445 & 2.051 & -2.024 \mathrm{H}\end{array}$

$\begin{array}{lllllllllllll}\text { HETATM } & 26 & \mathrm{H} & 0 & -2.922 & -0.231 & -2.009 \mathrm{H}\end{array}$

$\begin{array}{llllllll}\text { HETATM } & 27 & W & 0 & 0.017 & -0.48 & -0.229 & W\end{array}$

$\begin{array}{lllllllll}\text { HETATM } & 28 & P & 0 & 2.558 & -0.934 & 0.153 & P\end{array}$

$\begin{array}{llllllllll}\text { HETATM } & 29 & \mathrm{C} & 0 & 3.098 & -1.995 & 1.567 & \mathrm{C}\end{array}$

$\begin{array}{llllllllll}\text { HETATM } & 30 & \mathrm{H} & 0 & 2.642 & -2.986 & 1.525 & \mathrm{H}\end{array}$

$\begin{array}{llllllllll}\text { HETATM } & 31 & \mathrm{H} & 0 & 2.836 & -1.524 & 2.517 & \mathrm{H}\end{array}$

$\begin{array}{llllllll}\text { HETATM } & 32 & \mathrm{H} & 0 & 4.187 & -2.11 & 1.53 & \mathrm{H}\end{array}$

$\begin{array}{lllllllll}\text { HETATM } & 33 & \mathrm{C} & 0 & 3.333 & -1.783 & -1.288 \mathrm{C}\end{array}$

$\begin{array}{lllllll}\text { HETATM } & 34 & \mathrm{H} & 0 & 3.19 & -1.202 & -2.203 \mathrm{H}\end{array}$

$\begin{array}{llllllll}\text { HETATM } & 35 & \mathrm{H} & 0 & 2.858 & -2.758 & -1.435 \mathrm{H}\end{array}$

$\begin{array}{lllllllll}\text { HETATM } & 36 & \mathrm{H} & 0 & 4.405 & -1.928 & -1.121 \mathrm{H}\end{array}$

$\begin{array}{llllllll}\text { HETATM } & 37 & \mathrm{C} & 0 & 3.611 & 0.555 & 0.442 & \mathrm{C}\end{array}$

$\begin{array}{llllllll}\text { HETATM } & 38 & \mathrm{H} & 0 & 3.592 & 1.228 & -0.417 \mathrm{H}\end{array}$

$\begin{array}{llllllll}\text { HETATM } & 39 & \mathrm{H} & 0 & 4.643 & 0.243 & 0.63 & \mathrm{H}\end{array}$

$\begin{array}{llllllll}\text { HETATM } & 40 & \mathrm{H} & 0 & 3.238 & 1.097 & 1.317 & \mathrm{H}\end{array}$

$\begin{array}{llllllll}\text { HETATM } & 41 & \mathrm{~N} & 0 & 0.117 & -1.186 & -1.866 \mathrm{~N}\end{array}$

$\begin{array}{lllllllllll}\text { HETATM } & 42 & 0 & 0 & 0.254 & -1.605 & -2.976 & 0\end{array}$ 


\begin{tabular}{|c|c|c|c|c|c|c|}
\hline HETATM & 43 & C & 0 & -1.314 & -2.532 & 0.275 \\
\hline HETATM & 44 & C & 0 & 0.029 & -2.427 & 0.845 \\
\hline HETATM & 45 & C & 0 & -2.399 & -1.855 & 0.816 \\
\hline HETATM & 46 & $\mathrm{H}$ & 0 & 0.696 & -3.213 & 0.497 \\
\hline HETATM & 47 & C & 0 & -1.495 & -3.469 & -0.911 \\
\hline HETATM & 48 & $\mathrm{H}$ & 0 & -2.045 & -3.029 & -1.747 \\
\hline HETATM & 49 & $\mathrm{H}$ & 0 & -0.529 & -3.794 & -1.301 \\
\hline HETATM & 50 & $\mathrm{H}$ & 0 & -2.043 & -4.362 & -0.587 \\
\hline HETATM & 51 & C & 0 & -3.761 & -1.924 & 0.175 \\
\hline HETATM & 52 & $\mathrm{H}$ & 0 & -3.744 & -2.174 & -0.886 \\
\hline HETATM & 53 & $\mathrm{H}$ & 0 & -4.35 & -2.701 & 0.683 \\
\hline HETATM & 54 & $\mathrm{H}$ & 0 & -4.305 & -0.982 & 0.309 \\
\hline HETATM & 55 & $\mathrm{H}$ & 0 & 0.097 & -2.307 & 1.926 \\
\hline HETATM & 56 & C & 0 & -2.448 & -1.234 & 2.19 \\
\hline HETATM & 57 & $\mathrm{H}$ & 0 & -1.558 & -1.407 & 2.79 \\
\hline HETATM & 58 & $\mathrm{H}$ & 0 & -2.626 & -0.154 & 2.138 \\
\hline HETATM & 59 & $\mathrm{H}$ & 0 & -3.304 & -1.666 & 2.725 \\
\hline \multicolumn{7}{|l|}{ END } \\
\hline CONECT & 1 & 2 & 3 & 4 & 5 & \\
\hline CONECT & 2 & 1 & 6 & 9 & & \\
\hline CONECT & 3 & 1 & 14 & 17 & & \\
\hline CONECT & 4 & 1 & 10 & 13 & & \\
\hline CONECT & 5 & 1 & & & & \\
\hline CONECT & 6 & 2 & 7 & & & \\
\hline CONECT & 7 & 6 & 8 & 18 & & \\
\hline CONECT & 8 & 7 & 9 & 19 & & \\
\hline CONECT & 9 & 2 & 8 & 20 & & \\
\hline CONECT & 10 & 4 & 11 & 21 & & \\
\hline CONECT & 11 & 10 & 12 & 22 & & \\
\hline CONECT & 12 & 11 & 13 & 23 & & \\
\hline CONECT & 13 & 4 & 12 & & & \\
\hline CONECT & 14 & 3 & 15 & 24 & & \\
\hline CONECT & 15 & 14 & 16 & 25 & & \\
\hline CONECT & 16 & 15 & 17 & 26 & & \\
\hline CONECT & 17 & 3 & 16 & & & \\
\hline CONECT & 18 & 7 & & & & \\
\hline CONECT & 19 & 8 & & & & \\
\hline CONECT & 20 & 9 & & & & \\
\hline CONECT & 21 & 10 & & & & \\
\hline CONECT & 22 & 11 & & & & \\
\hline CONECT & 23 & 12 & & & & \\
\hline CONECT & 24 & 14 & & & & \\
\hline CONECT & 25 & 15 & & & & \\
\hline CONECT & 26 & 16 & & & & \\
\hline
\end{tabular}




\begin{tabular}{|c|c|c|c|c|c|}
\hline CONECT & 27 & 41 & & & \\
\hline CONECT & 28 & 29 & 33 & 37 & \\
\hline CONECT & 29 & 28 & 30 & 31 & 3. \\
\hline CONECT & 30 & 29 & & & \\
\hline CONECT & 31 & 29 & & & \\
\hline CONECT & 32 & 29 & & & \\
\hline CONECT & 33 & 28 & 34 & 35 & 3 \\
\hline CONECT & 34 & 33 & & & \\
\hline CONECT & 35 & 33 & & & \\
\hline CONECT & 36 & 33 & & & \\
\hline CONECT & 37 & 28 & 38 & 39 & 4 \\
\hline CONECT & 38 & 37 & & & \\
\hline CONECT & 39 & 37 & & & \\
\hline CONECT & 40 & 37 & & & \\
\hline CONECT & 41 & 27 & 42 & & \\
\hline CONECT & 42 & 41 & & & \\
\hline CONECT & 43 & 44 & 45 & 47 & \\
\hline CONECT & 44 & 43 & 46 & 55 & \\
\hline CONECT & 45 & 43 & 51 & 56 & \\
\hline CONECT & 46 & 44 & & & \\
\hline CONECT & 47 & 43 & 48 & 49 & 5 \\
\hline CONECT & 48 & 47 & & & \\
\hline CONECT & 49 & 47 & & & \\
\hline CONECT & 50 & 47 & & & \\
\hline CONECT & 51 & 45 & 52 & 53 & 5 \\
\hline CONECT & 52 & 51 & & & \\
\hline CONECT & 53 & 51 & & & \\
\hline CONECT & 54 & 51 & & & \\
\hline CONECT & 55 & 44 & & & \\
\hline CONECT & 56 & 45 & 57 & 58 & 5 \\
\hline CONECT & 57 & 56 & & & \\
\hline CONECT & 58 & 56 & & & \\
\hline CONECT & 59 & 56 & & & \\
\hline
\end{tabular}

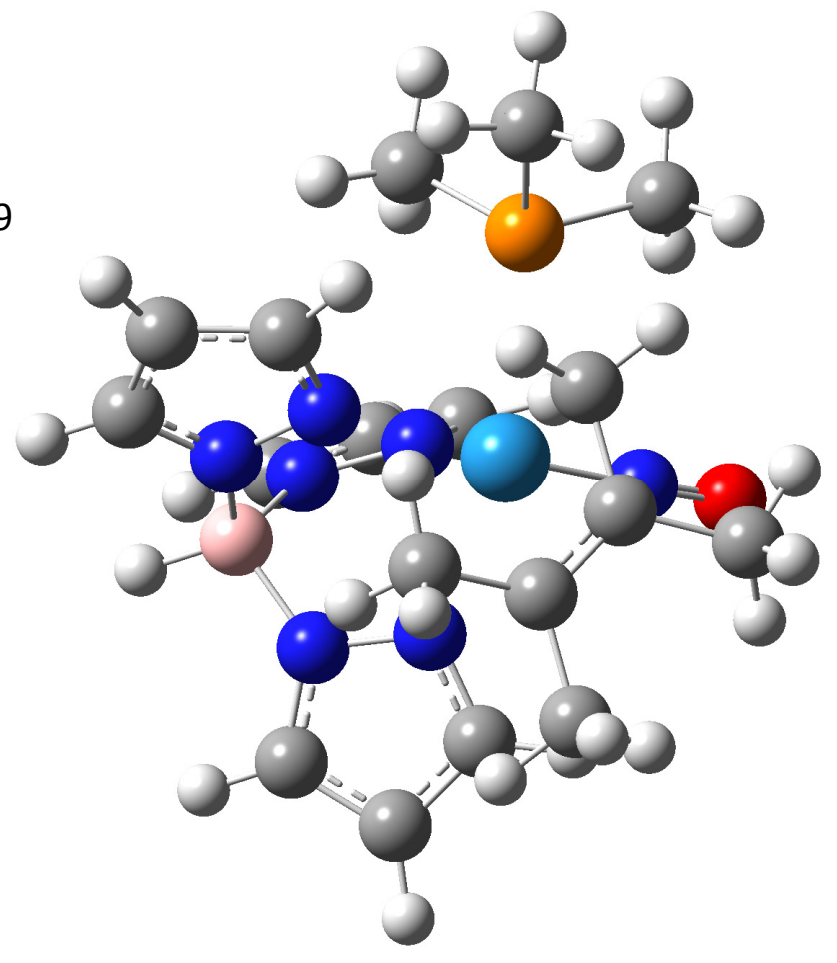




\begin{tabular}{|c|c|c|c|c|c|c|c|}
\hline TITLE & $12 \kappa$ & & & & & & \\
\hline REMARK & 1 & & $a t$ & y Gauss & View 5. & 0.8 & \\
\hline HETATM & 1 & B & 0 & -2.655 & -1.578 & 0.265 & B \\
\hline HETATM & 2 & $N$ & 0 & -2.817 & -0.482 & -0.822 & $\mathrm{~N}$ \\
\hline HETATM & 3 & $\mathrm{~N}$ & 0 & -1.501 & -2.485 & -0.214 & $\mathrm{~N}$ \\
\hline HETATM & 4 & $\mathrm{~N}$ & 0 & -2.256 & -0.908 & 1.599 & $\mathrm{~N}$ \\
\hline HETATM & 5 & $\mathrm{H}$ & 0 & -3.669 & -2.197 & 0.395 & $\mathrm{H}$ \\
\hline HETATM & 6 & $N$ & 0 & -1.724 & 0.229 & -1.22 & $\mathrm{~N}$ \\
\hline HETATM & 7 & C & 0 & -2.078 & 0.912 & -2.329 & C \\
\hline HETATM & 8 & C & 0 & -3.415 & 0.664 & -2.637 & C \\
\hline HETATM & 9 & C & 0 & -3.84 & -0.236 & -1.662 & C \\
\hline HETATM & 10 & C & 0 & -2.846 & -0.994 & 2.807 & C \\
\hline HETATM & 11 & C & 0 & -2.093 & -0.271 & 3.725 & C \\
\hline HETATM & 12 & C & 0 & -1.03 & 0.239 & 2.977 & C \\
\hline HETATM & 13 & $N$ & 0 & -1.13 & -0.146 & 1.689 & $\mathrm{~N}$ \\
\hline HETATM & 14 & C & 0 & -1.511 & -3.792 & -0.555 & C \\
\hline HETATM & 15 & C & 0 & -0.272 & -4.122 & -1.087 & C \\
\hline HETATM & 16 & C & 0 & 0.461 & -2.932 & -1.043 & C \\
\hline HETATM & 17 & $N$ & 0 & -0.283 & -1.95 & -0.512 & $\mathrm{~N}$ \\
\hline HETATM & 18 & $\mathrm{H}$ & 0 & -1.35 & 1.52 & -2.847 & $\mathrm{H}$ \\
\hline HETATM & 19 & $\mathrm{H}$ & 0 & -3.99 & 1.062 & -3.459 & $\mathrm{H}$ \\
\hline HETATM & 20 & $\mathrm{H}$ & 0 & -4.793 & -0.723 & -1.52 & $\mathrm{H}$ \\
\hline HETATM & 21 & $\mathrm{H}$ & 0 & -3.756 & -1.564 & 2.932 & $\mathrm{H}$ \\
\hline HETATM & 22 & $\mathrm{H}$ & 0 & -2.287 & -0.139 & 4.779 & $\mathrm{H}$ \\
\hline HETATM & 23 & $\mathrm{H}$ & 0 & -0.198 & 0.847 & 3.302 & $\mathrm{H}$ \\
\hline HETATM & 24 & $\mathrm{H}$ & 0 & -2.398 & -4.39 & -0.4 & $\mathrm{H}$ \\
\hline HETATM & 25 & $\mathrm{H}$ & 0 & 0.05 & -5.084 & -1.456 & $\mathrm{H}$ \\
\hline HETATM & 26 & $\mathrm{H}$ & 0 & 1.474 & -2.731 & -1.36 & $\mathrm{H}$ \\
\hline HETATM & 27 & W & 0 & 0.157 & 0.199 & -0.124 & w \\
\hline HETATM & 28 & $P$ & 0 & -0.049 & 2.746 & 0.284 & $\mathrm{P}$ \\
\hline HETATM & 29 & C & 0 & 0.961 & 3.465 & 1.653 & C \\
\hline HETATM & 30 & $\mathrm{H}$ & 0 & 2.026 & 3.302 & 1.464 & $\mathrm{H}$ \\
\hline HETATM & 31 & $\mathrm{H}$ & 0 & 0.699 & 2.997 & 2.605 & $\mathrm{H}$ \\
\hline HETATM & 32 & $\mathrm{H}$ & 0 & 0.778 & 4.542 & 1.729 & $\mathrm{H}$ \\
\hline HETATM & 33 & C & 0 & 0.416 & 3.772 & -1.173 & C \\
\hline HETATM & 34 & $\mathrm{H}$ & 0 & -0.262 & 3.565 & -2.005 & $\mathrm{H}$ \\
\hline HETATM & 35 & $\mathrm{H}$ & 0 & 1.431 & 3.524 & -1.494 & $\mathrm{H}$ \\
\hline HETATM & 36 & $\mathrm{H}$ & 0 & 0.359 & 4.838 & -0.929 & $\mathrm{H}$ \\
\hline HETATM & 37 & C & 0 & -1.767 & 3.274 & 0.69 & C \\
\hline HETATM & 38 & $\mathrm{H}$ & 0 & -2.444 & 2.971 & -0.114 & $\mathrm{H}$ \\
\hline HETATM & 39 & $\mathrm{H}$ & 0 & -1.816 & 4.361 & 0.812 & $\mathrm{H}$ \\
\hline HETATM & 40 & $\mathrm{H}$ & 0 & -2.092 & 2.79 & 1.615 & $\mathrm{H}$ \\
\hline HETATM & 41 & $N$ & 0 & 1.105 & 0.518 & -1.609 & $\mathrm{~N}$ \\
\hline HETATM & 42 & 0 & 0 & 1.69 & 0.766 & -2.62 & \\
\hline
\end{tabular}




$\begin{array}{llllllll}\text { HETATM } & 43 & \mathrm{C} & 0 & 3.1 & -1.145 & 0.677 & \mathrm{C} \\ \text { HETATM } & 44 & \mathrm{C} & 0 & 1.753 & -0.641 & 0.976 & \mathrm{C} \\ \text { HETATM } & 45 & \mathrm{C} & 0 & 4.108 & -0.409 & 0.124 & \mathrm{C} \\ \text { HETATM } & 46 & \mathrm{H} & 0 & 1.336 & -1.135 & 1.858 & \mathrm{H} \\ \text { HETATM } & 47 & \mathrm{H} & 0 & 1.715 & 0.507 & 1.144 & \mathrm{H} \\ \text { HETATM } & 48 & \mathrm{C} & 0 & 3.324 & -2.594 & 1.1 & \mathrm{C} \\ \text { HETATM } & 49 & \mathrm{H} & 0 & 2.397 & -3.051 & 1.455 & \mathrm{H} \\ \text { HETATM } & 50 & \mathrm{H} & 0 & 3.686 & -3.21 & 0.27 & \mathrm{H} \\ \text { HETATM } & 51 & \mathrm{H} & 0 & 4.061 & -2.673 & 1.908 & \mathrm{H} \\ \text { HETATM } & 52 & \mathrm{C} & 0 & 4.012 & 1.047 & -0.255 & \mathrm{C} \\ \text { HETATM } & 53 & \mathrm{H} & 0 & 3.857 & 1.156 & -1.336 & \mathrm{H} \\ \text { HETATM } & 54 & \mathrm{H} & 0 & 3.198 & 1.581 & 0.245 & \mathrm{H} \\ \text { HETATM } & 55 & \mathrm{H} & 0 & 4.949 & 1.562 & -0.012 & \mathrm{H} \\ \text { HETATM } & 56 & \mathrm{C} & 0 & 5.478 & -0.986 & -0.126 & \mathrm{C} \\ \text { HETATM } & 57 & \mathrm{H} & 0 & 5.532 & -2.069 & -0.01 & \mathrm{H} \\ \text { HETATM } & 58 & \mathrm{H} & 0 & 5.816 & -0.734 & -1.14 & \mathrm{H} \\ \text { HETATM } & 59 & \mathrm{H} & 0 & 6.21 & -0.537 & 0.56 & \mathrm{H} \\ \text { END } & & & & & & & \\ \text { CONECT } & 1 & 2 & 3 & 4 & 5 & & \\ \text { CONECT } & 2 & 1 & 6 & 9 & & & \\ \text { CONECT } & 3 & 1 & 14 & 17 & & & \\ \text { CONECT } & 4 & 1 & 10 & 13 & & & \\ \text { CONECT } & 5 & 1 & & & & & \\ \text { CONECT } & 6 & 2 & 7 & & & & \\ \text { CONECT } & 7 & 6 & 8 & 18 & & & \\ \text { CONECT } & 8 & 7 & 9 & 19 & & & \\ \text { CONECT } & 9 & 2 & 8 & 20 & & & \\ \text { CONECT } & 10 & 4 & 11 & 21 & & & \\ \text { CONECT } & 11 & 10 & 12 & 22 & & & \\ \text { CONECT } & 12 & 11 & 13 & 23 & & & \\ \text { CONECT } & 13 & 4 & 12 & & & & \\ \text { CONECT } & 14 & 3 & 15 & 24 & & & \\ \text { CONECT } & 15 & 14 & 16 & 25 & & & \\ \text { CONECT } & 16 & 15 & 17 & 26 & & & \\ \text { CONECT } & 17 & 16 & 3 & & & & \\ \text { CONECT } & 18 & 7 & & & & & \\ \text { CONECT } & 19 & 8 & & & & & \\ \text { CONECT } & 20 & 9 & & & & & \\ \text { CONECT } & 21 & 10 & & & & & \\ \text { CONECT } & 22 & 11 & & & & & \\ \text { CONECT } & 23 & 12 & & & & & \\ \text { CONECT } & 24 & 14 & & & & & \\ \text { CONECT } & 25 & 15 & & & & & \\ \text { CONECT } & 26 & 16 & & & & & \end{array}$




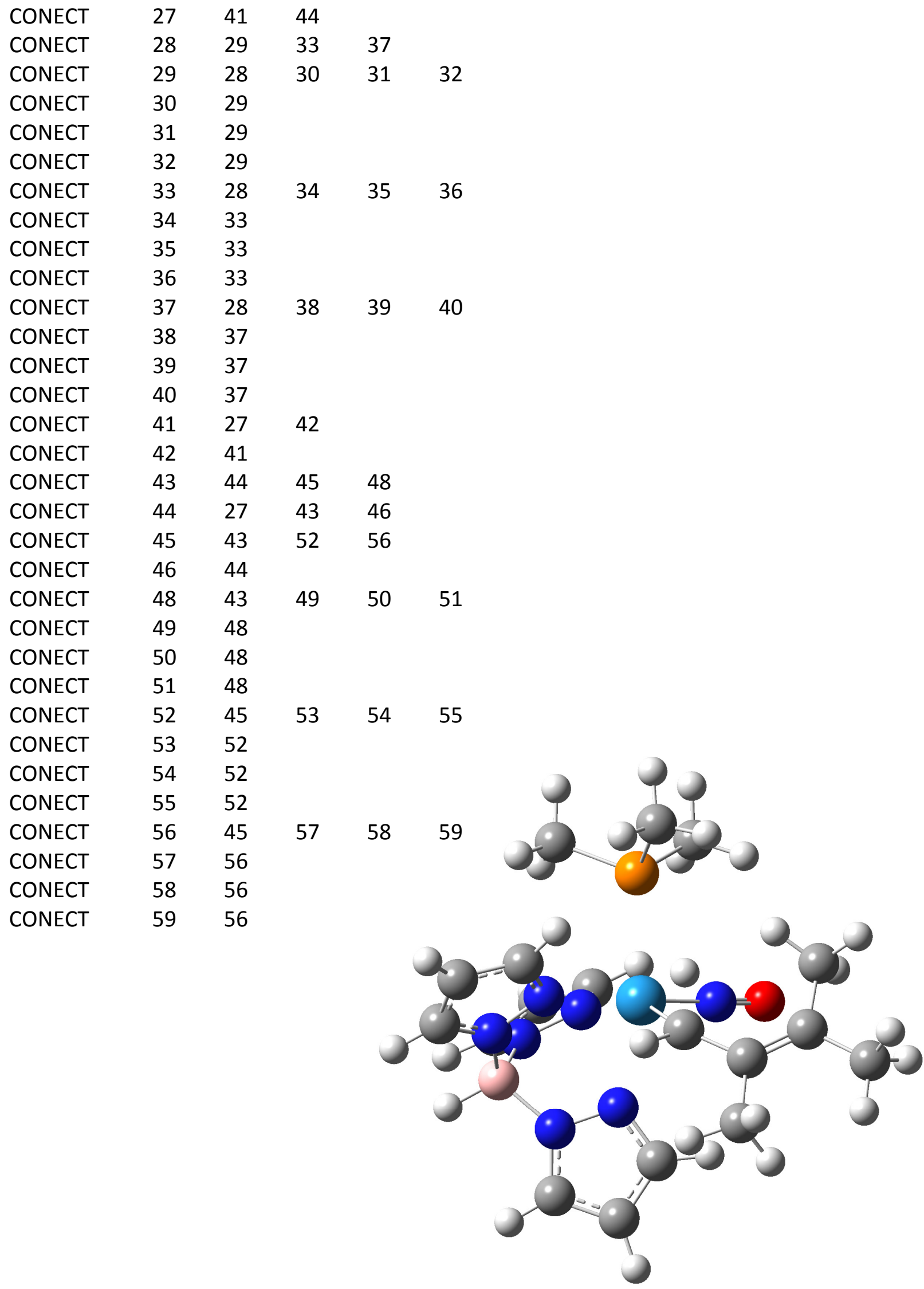




\begin{tabular}{|c|c|c|c|c|c|c|c|}
\hline TITLE & $12^{\circ}$ & & & & & & \\
\hline REMARK & 1 & & eat & y Gauss & View 5. & 0.8 & \\
\hline HETATM & 1 & B & 0 & -1.49 & -2.578 & 0.236 & B \\
\hline HETATM & 2 & $N$ & 0 & -2.361 & -1.717 & -0.709 & $\mathrm{~N}$ \\
\hline HETATM & 3 & $N$ & 0 & -0.089 & -2.728 & -0.398 & $\mathrm{~N}$ \\
\hline HETATM & 4 & $\mathrm{~N}$ & 0 & -1.36 & -1.849 & 1.592 & $\mathrm{~N}$ \\
\hline HETATM & 5 & $\mathrm{H}$ & 0 & -1.992 & -3.658 & 0.394 & $\mathrm{H}$ \\
\hline HETATM & 6 & $N$ & 0 & -1.951 & -0.47 & -1.067 & $\mathrm{~N}$ \\
\hline HETATM & 7 & C & 0 & -2.829 & -0.023 & -1.978 & C \\
\hline HETATM & 8 & C & 0 & -3.826 & -0.981 & -2.211 & C \\
\hline HETATM & 9 & C & 0 & -3.484 & -2.043 & -1.386 & C \\
\hline HETATM & 10 & C & 0 & -1.738 & -2.256 & 2.822 & C \\
\hline HETATM & 11 & C & 0 & -1.411 & -1.262 & 3.734 & C \\
\hline HETATM & 12 & C & 0 & -0.819 & -0.258 & 2.957 & C \\
\hline HETATM & 13 & $\mathrm{~N}$ & 0 & -0.792 & -0.615 & 1.664 & $\mathrm{~N}$ \\
\hline HETATM & 14 & C & 0 & 0.53 & -3.844 & -0.841 & C \\
\hline HETATM & 15 & C & 0 & 1.727 & -3.481 & -1.442 & C \\
\hline HETATM & 16 & C & 0 & 1.771 & -2.086 & -1.33 & C \\
\hline HETATM & 17 & $N$ & 0 & 0.677 & -1.636 & -0.697 & $\mathrm{~N}$ \\
\hline HETATM & 18 & $\mathrm{H}$ & 0 & -2.687 & 0.948 & -2.431 & $\mathrm{H}$ \\
\hline HETATM & 19 & $\mathrm{H}$ & 0 & -4.665 & -0.914 & -2.887 & $\mathrm{H}$ \\
\hline HETATM & 20 & $\mathrm{H}$ & 0 & -3.951 & -3.006 & -1.237 & $\mathrm{H}$ \\
\hline HETATM & 21 & $\mathrm{H}$ & 0 & -2.206 & -3.22 & 2.959 & $\mathrm{H}$ \\
\hline HETATM & 22 & $\mathrm{H}$ & 0 & -1.571 & -1.267 & 4.802 & $\mathrm{H}$ \\
\hline HETATM & 23 & $\mathrm{H}$ & 0 & -0.397 & 0.687 & 3.265 & $\mathrm{H}$ \\
\hline HETATM & 24 & $\mathrm{H}$ & 0 & 0.071 & -4.812 & -0.7 & $\mathrm{H}$ \\
\hline HETATM & 25 & $\mathrm{H}$ & 0 & 2.461 & -4.131 & -1.893 & $\mathrm{H}$ \\
\hline HETATM & 26 & $\mathrm{H}$ & 0 & 2.531 & -1.384 & -1.638 & $\mathrm{H}$ \\
\hline HETATM & 27 & W & 0 & -0.006 & 0.411 & -0.234 & w \\
\hline HETATM & 28 & $P$ & 0 & -1.135 & 2.587 & 0.395 & $P$ \\
\hline HETATM & 29 & C & 0 & -0.216 & 3.749 & 1.505 & C \\
\hline HETATM & 30 & $\mathrm{H}$ & 0 & 0.726 & 4.035 & 1.03 & $\mathrm{H}$ \\
\hline HETATM & 31 & $\mathrm{H}$ & 0 & 0.023 & 3.257 & 2.452 & $\mathrm{H}$ \\
\hline HETATM & 32 & $\mathrm{H}$ & 0 & -0.808 & 4.649 & 1.704 & $\mathrm{H}$ \\
\hline HETATM & 33 & C & 0 & -1.509 & 3.664 & -1.06 & C \\
\hline HETATM & 34 & $\mathrm{H}$ & 0 & -2.303 & 3.209 & -1.659 & $\mathrm{H}$ \\
\hline HETATM & 35 & $\mathrm{H}$ & 0 & -0.617 & 3.738 & -1.69 & $\mathrm{H}$ \\
\hline HETATM & 36 & $\mathrm{H}$ & 0 & -1.831 & 4.663 & -0.748 & $\mathrm{H}$ \\
\hline HETATM & 37 & C & 0 & -2.781 & 2.462 & 1.235 & C \\
\hline HETATM & 38 & $\mathrm{H}$ & 0 & -3.461 & 1.879 & 0.606 & $\mathrm{H}$ \\
\hline HETATM & 39 & $\mathrm{H}$ & 0 & -3.215 & 3.452 & 1.415 & $\mathrm{H}$ \\
\hline HETATM & 40 & $\mathrm{H}$ & 0 & -2.672 & 1.937 & 2.188 & $\mathrm{H}$ \\
\hline HETATM & 41 & $\mathrm{~N}$ & 0 & 0.553 & 1.216 & -1.725 & IN \\
\hline HETATM & 42 & 0 & 0 & 0.903 & 1.809 & -2.722 & \\
\hline
\end{tabular}




$\begin{array}{llllllll}\text { HETATM } & 43 & \mathrm{C} & 0 & 3.009 & 0.132 & 0.902 & \mathrm{C} \\ \text { HETATM } & 44 & \mathrm{C} & 0 & 1.787 & 1.016 & 0.98 & \mathrm{C} \\ \text { HETATM } & 45 & \mathrm{C} & 0 & 4.05 & 0.357 & 0.064 & \mathrm{C} \\ \text { HETATM } & 46 & \mathrm{H} & 0 & 1.474 & 1.048 & 2.036 & \mathrm{H} \\ \text { HETATM } & 47 & \mathrm{H} & 0 & 2.055 & 2.045 & 0.717 & \mathrm{H} \\ \text { HETATM } & 48 & \mathrm{C} & 0 & 2.975 & -1.02 & 1.889 & \mathrm{C} \\ \text { HETATM } & 49 & \mathrm{H} & 0 & 1.998 & -1.518 & 1.855 & \mathrm{H} \\ \text { HETATM } & 50 & \mathrm{H} & 0 & 3.738 & -1.782 & 1.717 & \mathrm{H} \\ \text { HETATM } & 51 & \mathrm{H} & 0 & 3.098 & -0.65 & 2.918 & \mathrm{H} \\ \text { HETATM } & 52 & \mathrm{C} & 0 & 4.077 & 1.519 & -0.91 & \mathrm{C} \\ \text { HETATM } & 53 & \mathrm{H} & 0 & 4.593 & 1.232 & -1.837 & \mathrm{H} \\ \text { HETATM } & 54 & \mathrm{H} & 0 & 3.084 & 1.872 & -1.193 & \mathrm{H} \\ \text { HETATM } & 55 & \mathrm{H} & 0 & 4.639 & 2.374 & -0.501 & \mathrm{H} \\ \text { HETATM } & 56 & \mathrm{C} & 0 & 5.323 & -0.461 & 0.024 & \mathrm{C} \\ \text { HETATM } & 57 & \mathrm{H} & 0 & 6.198 & 0.189 & 0.182 & \mathrm{H} \\ \text { HETATM } & 58 & \mathrm{H} & 0 & 5.371 & -1.253 & 0.774 & \mathrm{H} \\ \text { HETATM } & 59 & \mathrm{H} & 0 & 5.47 & -0.926 & -0.963 & \mathrm{H} \\ \text { END } & & & & & & & \\ \text { CONECT } & 1 & 2 & 3 & 4 & 5 & & \\ \text { CONECT } & 2 & 1 & 6 & 9 & & & \\ \text { CONECT } & 3 & 1 & 14 & 17 & & & \\ \text { CONECT } & 4 & 1 & 10 & 13 & & & \\ \text { CONECT } & 5 & 1 & & & & & \\ \text { CONECT } & 6 & 2 & 7 & & & & \\ \text { CONECT } & 7 & 6 & 8 & 18 & & & \\ \text { CONECT } & 8 & 7 & 9 & 19 & & & \\ \text { CONECT } & 9 & 2 & 8 & 20 & & & \\ \text { CONECT } & 10 & 4 & 11 & 21 & & & \\ \text { CONECT } & 11 & 10 & 12 & 22 & & & \\ \text { CONECT } & 12 & 11 & 13 & 23 & & & \\ \text { CONECT } & 13 & 4 & 12 & & & & \\ \text { CONECT } & 14 & 3 & 15 & 24 & & & \\ \text { CONECT } & 15 & 14 & 16 & 25 & & & \\ \text { CONECT } & 16 & 15 & 17 & 26 & & & \\ \text { CONECT } & 17 & 3 & 16 & & & & \\ \text { CONECT } & 18 & 7 & & & & & \\ \text { CONECT } & 19 & 8 & & & & & \\ \text { CONECT } & 20 & 9 & & & & & \\ \text { CONECT } & 21 & 10 & & & & & \\ \text { CONECT } & 22 & 11 & & & & & \\ \text { CONECT } & 23 & 12 & & & & & \\ \text { CONECT } & 24 & 14 & & & & & \\ \text { CONECT } & 25 & 15 & & & & & \\ \text { CONECT } & 26 & 16 & & & & & \end{array}$




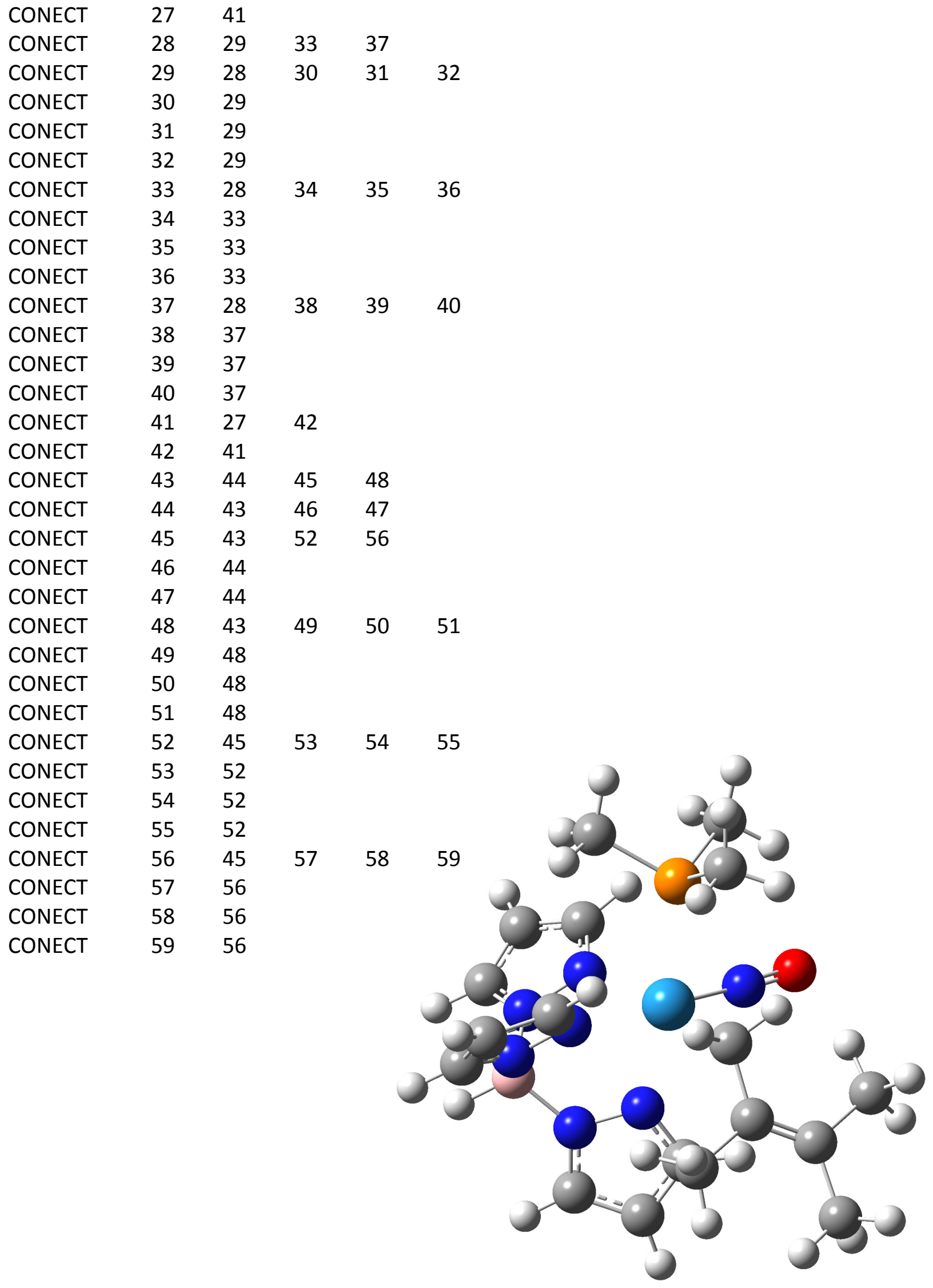




\begin{tabular}{|c|c|c|c|c|c|c|c|}
\hline TITLE & $12^{-}$ & & & & & & \\
\hline REMARK & 1 & & eat & y Gauss & View 5. & 0.8 & \\
\hline HETATM & 1 & $B$ & 0 & -1.522 & -2.56 & 0.236 & B \\
\hline HETATM & 2 & $\mathrm{~N}$ & 0 & -2.365 & -1.695 & -0.715 & $\mathrm{~N}$ \\
\hline HETATM & 3 & $\mathrm{~N}$ & 0 & -0.115 & -2.724 & -0.371 & $\mathrm{~N}$ \\
\hline HETATM & 4 & $\mathrm{~N}$ & 0 & -1.406 & -1.844 & 1.607 & $\mathrm{~N}$ \\
\hline HETATM & 5 & $\mathrm{H}$ & 0 & -2.038 & -3.639 & 0.391 & $\mathrm{H}$ \\
\hline HETATM & 6 & $\mathrm{~N}$ & 0 & -1.934 & -0.448 & -1.069 & $\mathrm{~N}$ \\
\hline HETATM & 7 & C & 0 & -2.767 & -0.024 & -2.035 & $C$ \\
\hline HETATM & 8 & C & 0 & -3.746 & -0.995 & -2.31 & C \\
\hline HETATM & 9 & C & 0 & -3.458 & -2.039 & -1.447 & $C$ \\
\hline HETATM & 10 & C & 0 & -1.779 & -2.267 & 2.834 & C \\
\hline HETATM & 11 & C & 0 & -1.445 & -1.285 & 3.758 & C \\
\hline HETATM & 12 & C & 0 & -0.853 & -0.273 & 2.986 & C \\
\hline HETATM & 13 & $\mathrm{~N}$ & 0 & -0.837 & -0.614 & 1.691 & $\mathrm{~N}$ \\
\hline HETATM & 14 & C & 0 & 0.483 & -3.847 & -0.843 & $C$ \\
\hline HETATM & 15 & C & 0 & 1.668 & -3.484 & -1.461 & C \\
\hline HETATM & 16 & C & 0 & 1.732 & -2.087 & -1.319 & \\
\hline HETATM & 17 & $\mathrm{~N}$ & 0 & 0.655 & -1.631 & -0.662 & $\mathrm{~N}$ \\
\hline HETATM & 18 & $\mathrm{H}$ & 0 & -2.61 & 0.948 & -2.479 & $\mathrm{H}$ \\
\hline HETATM & 19 & $\mathrm{H}$ & 0 & -4.552 & -0.937 & -3.028 & $\mathrm{H}$ \\
\hline HETATM & 20 & $\mathrm{H}$ & 0 & -3.942 & -2.993 & -1.289 & $\mathrm{H}$ \\
\hline HETATM & 21 & $\mathrm{H}$ & 0 & -2.249 & -3.232 & 2.958 & $\mathrm{H}$ \\
\hline HETATM & 22 & $\mathrm{H}$ & 0 & -1.599 & -1.303 & 4.827 & $\mathrm{H}$ \\
\hline HETATM & 23 & $\mathrm{H}$ & 0 & -0.426 & 0.671 & 3.295 & $\mathrm{H}$ \\
\hline HETATM & 24 & $\mathrm{H}$ & 0 & 0.018 & -4.812 & -0.699 & $\mathrm{H}$ \\
\hline HETATM & 25 & $\mathrm{H}$ & 0 & 2.391 & -4.133 & -1.934 & $\mathrm{H}$ \\
\hline HETATM & 26 & $\mathrm{H}$ & 0 & 2.498 & -1.388 & -1.618 & $3 \mathrm{H}$ \\
\hline HETATM & 27 & W & 0 & -0.042 & 0.448 & -0.228 & 3 \\
\hline HETATM & 28 & $P$ & 0 & -1.025 & 2.546 & 0.4 & $r$ \\
\hline HETATM & 29 & C & 0 & -0.084 & 3.857 & 1.379 & C \\
\hline HETATM & 30 & $\mathrm{H}$ & 0 & 0.812 & 4.14 & 0.816 & $\mathrm{H}$ \\
\hline HETATM & 31 & $\mathrm{H}$ & 0 & 0.249 & 3.435 & 2.334 & $\mathrm{H}$ \\
\hline HETATM & 32 & $\mathrm{H}$ & 0 & -0.686 & 4.755 & 1.571 & $\mathrm{H}$ \\
\hline HETATM & 33 & C & 0 & -1.554 & 3.609 & -1.037 & $7 \mathrm{C}$ \\
\hline HETATM & 34 & $\mathrm{H}$ & 0 & -2.433 & 3.16 & -1.511 & $\mathrm{LH}$ \\
\hline HETATM & 35 & $\mathrm{H}$ & 0 & -0.745 & 3.619 & -1.774 & $\mathrm{H}$ \\
\hline HETATM & 36 & $\mathrm{H}$ & 0 & -1.801 & 4.635 & -0.734 & $+\mathrm{H}$ \\
\hline HETATM & 37 & C & 0 & -2.604 & 2.548 & 1.407 & C \\
\hline HETATM & 38 & $\mathrm{H}$ & 0 & -3.363 & 1.97 & 0.869 & $\mathrm{H}$ \\
\hline HETATM & 39 & $\mathrm{H}$ & 0 & -2.98 & 3.565 & 1.584 & $\mathrm{H}$ \\
\hline HETATM & 40 & $\mathrm{H}$ & 0 & -2.436 & 2.06 & 2.372 & $\mathrm{H}$ \\
\hline HETATM & 41 & $\mathrm{~N}$ & 0 & 0.513 & 1.242 & -1.723 & $\mathrm{~N}$ \\
\hline HETATM & 42 & 0 & 0 & 0.865 & 1.833 & -2.745 & \\
\hline
\end{tabular}




\begin{tabular}{|c|c|c|c|c|c|c|}
\hline HETATM & 43 & C & 0 & 3.039 & 0.051 & 0.906 \\
\hline HETATM & 44 & C & 0 & 1.838 & 0.939 & 1.021 \\
\hline HETATM & 45 & $C$ & 0 & 4.071 & 0.28 & 0.052 \\
\hline HETATM & 46 & $\mathrm{H}$ & 0 & 1.521 & 0.939 & 2.078 \\
\hline HETATM & 47 & $\mathrm{H}$ & 0 & 2.115 & 1.975 & 0.795 \\
\hline HETATM & 48 & $C$ & 0 & 3.006 & -1.138 & 1.849 \\
\hline HETATM & 49 & $\mathrm{H}$ & 0 & 2.01 & -1.594 & 1.83 \\
\hline HETATM & 50 & $\mathrm{H}$ & 0 & 3.731 & -1.923 & 1.616 \\
\hline HETATM & 51 & $\mathrm{H}$ & 0 & 3.183 & -0.816 & 2.887 \\
\hline HETATM & 52 & $C$ & 0 & 4.073 & 1.48 & -0.878 \\
\hline HETATM & 53 & $\mathrm{H}$ & 0 & 4.728 & 1.302 & -1.743 \\
\hline HETATM & 54 & $\mathrm{H}$ & 0 & 3.077 & 1.712 & -1.266 \\
\hline HETATM & 55 & $\mathrm{H}$ & 0 & 4.454 & 2.39 & -0.383 \\
\hline HETATM & 56 & $C$ & 0 & 5.325 & -0.56 & -0.053 \\
\hline HETATM & 57 & $\mathrm{H}$ & 0 & 5.352 & -1.408 & 0.637 \\
\hline HETATM & 58 & $\mathrm{H}$ & 0 & 5.463 & -0.962 & -1.072 \\
\hline HETATM & 59 & $\mathrm{H}$ & 0 & 6.225 & 0.047 & 0.148 \\
\hline \multicolumn{7}{|l|}{ END } \\
\hline CONECT & 1 & 2 & 3 & 4 & 5 & \\
\hline CONECT & 2 & 1 & 6 & 9 & & \\
\hline CONECT & 3 & 1 & 14 & 17 & & \\
\hline CONECT & 4 & 1 & 10 & 13 & & \\
\hline CONECT & 5 & 1 & & & & \\
\hline CONECT & 6 & 2 & 7 & & & \\
\hline CONECT & 7 & 6 & 8 & 18 & & \\
\hline CONECT & 8 & 7 & 9 & 19 & & \\
\hline CONECT & 9 & 2 & 8 & 20 & & \\
\hline CONECT & 10 & 4 & 11 & 21 & & \\
\hline CONECT & 11 & 10 & 12 & 22 & & \\
\hline CONECT & 12 & 11 & 13 & 23 & & \\
\hline CONECT & 13 & 4 & 12 & & & \\
\hline CONECT & 14 & 3 & 15 & 24 & & \\
\hline CONECT & 15 & 14 & 16 & 25 & & \\
\hline CONECT & 16 & 15 & 17 & 26 & & \\
\hline CONECT & 17 & 3 & 16 & & & \\
\hline CONECT & 18 & 7 & & & & \\
\hline CONECT & 19 & 8 & & & & \\
\hline CONECT & 20 & 9 & & & & \\
\hline CONECT & 21 & 10 & & & & \\
\hline CONECT & 22 & 11 & & & & \\
\hline CONECT & 23 & 12 & & & & \\
\hline CONECT & 24 & 14 & & & & \\
\hline CONECT & 25 & 15 & & & & \\
\hline CONECT & 26 & 16 & & & & \\
\hline
\end{tabular}




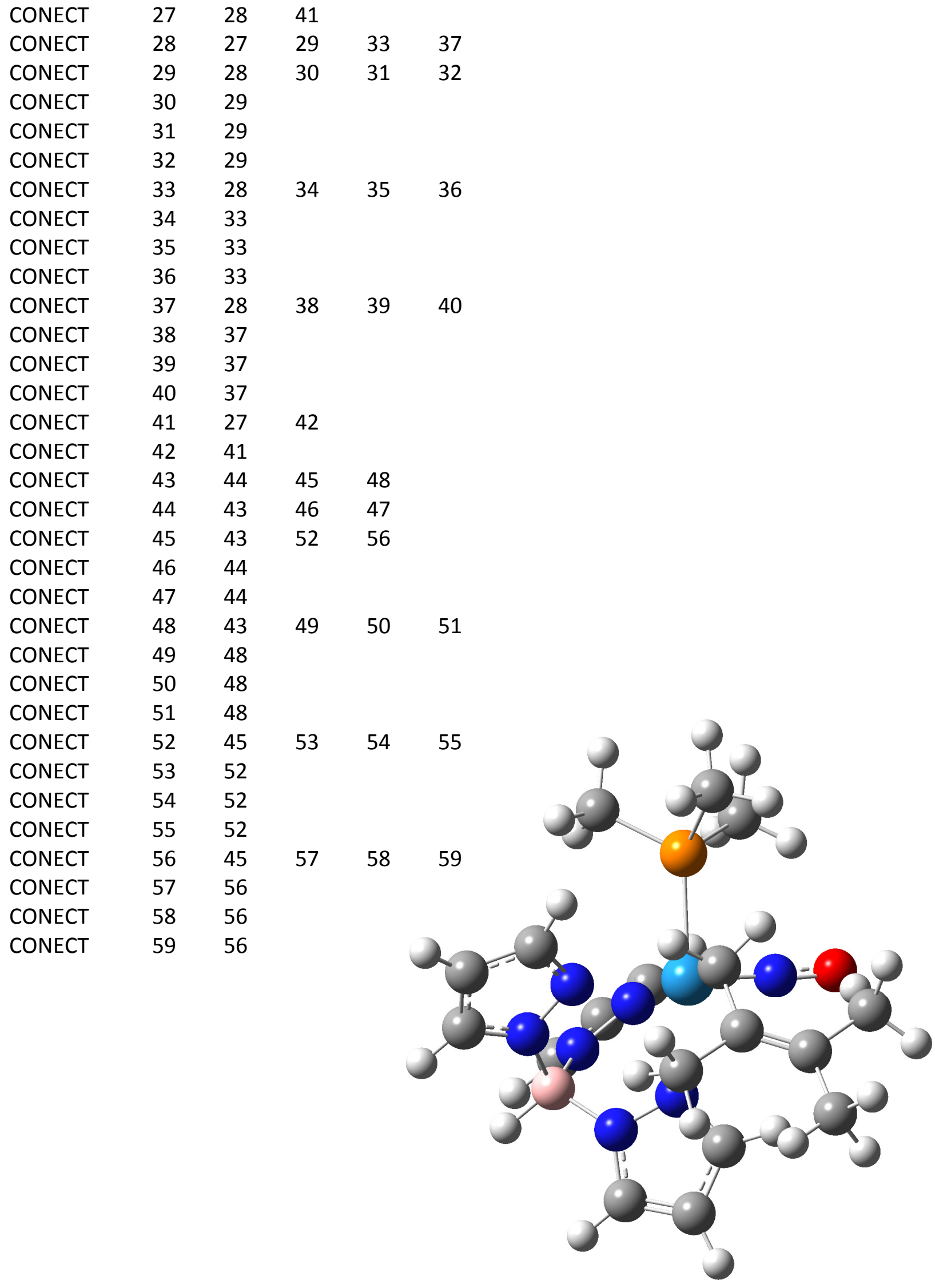




\begin{tabular}{|c|c|c|c|c|c|c|c|}
\hline TITLE & Exo & & & & & & \\
\hline REMARK & 1 & File & eat & y Gauss & View 5. & 0.8 & \\
\hline HETATM & 1 & $B$ & 0 & 3.199 & 0.353 & 0.225 & B \\
\hline HETATM & 2 & $\mathrm{~N}$ & 0 & 2.85 & -0.555 & -0.977 & $\mathrm{~N}$ \\
\hline HETATM & 3 & $N$ & 0 & 2.552 & 1.723 & -0.043 & $\mathrm{~N}$ \\
\hline HETATM & 4 & $\mathrm{~N}$ & 0 & 2.602 & -0.231 & 1.526 & $\mathrm{~N}$ \\
\hline HETATM & 5 & $\mathrm{H}$ & 0 & 4.39 & 0.456 & 0.336 & $\mathrm{H}$ \\
\hline HETATM & 6 & $N$ & 0 & 1.545 & -0.686 & -1.337 & $\mathrm{~N}$ \\
\hline HETATM & 7 & C & 0 & 1.53 & -1.193 & -2.579 & $C$ \\
\hline HETATM & 8 & C & 0 & 2.838 & -1.412 & -3.03 & C \\
\hline HETATM & 9 & C & 0 & 3.643 & -0.982 & -1.984 & C \\
\hline HETATM & 10 & C & 0 & 3.243 & -0.56 & 2.667 & C \\
\hline HETATM & 11 & C & 0 & 2.299 & -0.935 & 3.613 & C \\
\hline HETATM & 12 & C & 0 & 1.073 & -0.8 & 2.951 & C \\
\hline HETATM & 13 & $\mathrm{~N}$ & 0 & 1.257 & -0.376 & 1.69 & $\mathrm{~N}$ \\
\hline HETATM & 14 & C & 0 & 3.147 & 2.93 & -0.176 & $C$ \\
\hline HETATM & 15 & C & 0 & 2.171 & 3.877 & -0.438 & $C$ \\
\hline HETATM & 16 & C & 0 & 0.968 & 3.157 & -0.45 & C \\
\hline HETATM & 17 & $\mathrm{~N}$ & 0 & 1.202 & 1.858 & -0.213 & $\mathrm{~N}$ \\
\hline HETATM & 18 & $\mathrm{H}$ & 0 & 0.586 & -1.349 & -3.083 & $\mathrm{H}$ \\
\hline HETATM & 19 & $\mathrm{H}$ & 0 & 3.152 & -1.813 & -3.982 & \\
\hline HETATM & 20 & $\mathrm{H}$ & 0 & 4.718 & -0.934 & -1.888 & $\mathrm{H}$ \\
\hline HETATM & 21 & $\mathrm{H}$ & 0 & 4.319 & -0.495 & 2.724 & $\mathrm{H}$ \\
\hline HETATM & 22 & $\mathrm{H}$ & 0 & 2.472 & -1.251 & 4.631 & $\mathrm{H}$ \\
\hline HETATM & 23 & $\mathrm{H}$ & 0 & 0.077 & -0.978 & 3.327 & $\mathrm{H}$ \\
\hline HETATM & 24 & $\mathrm{H}$ & 0 & 4.219 & 3.02 & -0.071 & $\mathrm{H}$ \\
\hline HETATM & 25 & $\mathrm{H}$ & 0 & 2.304 & 4.937 & -0.592 & . $\mathrm{H}$ \\
\hline HETATM & 26 & $\mathrm{H}$ & 0 & -0.044 & 3.515 & -0.562 & H \\
\hline HETATM & 27 & W & 0 & -0.158 & 0.048 & -0.081 & L \\
\hline HETATM & 28 & $P$ & 0 & -0.819 & -2.406 & 0.002 & $r$ \\
\hline HETATM & 29 & C & 0 & -1.336 & -3.186 & 1.602 & C \\
\hline HETATM & 30 & $\mathrm{H}$ & 0 & -2.186 & -2.657 & 2.036 & $\mathrm{H}$ \\
\hline HETATM & 31 & $\mathrm{H}$ & 0 & -0.504 & -3.152 & 2.312 & $\mathrm{H}$ \\
\hline HETATM & 32 & $\mathrm{H}$ & 0 & -1.614 & -4.232 & 1.439 & $\mathrm{H}$ \\
\hline HETATM & 33 & C & 0 & -2.223 & -2.813 & -1.123 & $3 \mathrm{C}$ \\
\hline HETATM & 34 & $\mathrm{H}$ & 0 & -1.992 & -2.462 & -2.133 & $3 \mathrm{H}$ \\
\hline HETATM & 35 & $\mathrm{H}$ & 0 & -3.112 & -2.272 & -0.789 & $\mathrm{H}$ \\
\hline HETATM & 36 & $\mathrm{H}$ & 0 & -2.424 & -3.889 & -1.14 & $\mathrm{H}$ \\
\hline HETATM & 37 & C & 0 & 0.474 & -3.635 & -0.508 & $3 C$ \\
\hline HETATM & 38 & $\mathrm{H}$ & 0 & 0.777 & -3.476 & -1.545 & $\mathrm{H}$ \\
\hline HETATM & 39 & $\mathrm{H}$ & 0 & 0.094 & -4.657 & -0.399 & $\mathrm{H}$ \\
\hline HETATM & 40 & $\mathrm{H}$ & 0 & 1.36 & -3.514 & 0.124 & $\mathrm{H}$ \\
\hline HETATM & 41 & $N$ & 0 & -1.121 & 0.23 & -1.566 & $5 \mathrm{~N}$ \\
\hline HETATM & 42 & 0 & 0 & -1.727 & 0.266 & -2.614 & \\
\hline
\end{tabular}




\begin{tabular}{|c|c|c|c|c|c|c|}
\hline HETATM & 43 & C & 0 & -1.396 & 1.461 & 1.138 \\
\hline HETATM & 44 & C & 0 & -1.896 & 0.123 & 1.394 \\
\hline HETATM & 45 & C & 0 & -2.244 & 2.418 & 0.403 \\
\hline HETATM & 46 & $\mathrm{H}$ & 0 & -0.757 & 1.935 & 1.882 \\
\hline HETATM & 47 & $\mathrm{H}$ & 0 & -1.609 & -0.31 & 2.353 \\
\hline HETATM & 48 & C & 0 & -3.499 & 1.889 & -0.297 \\
\hline HETATM & 49 & $\mathrm{H}$ & 0 & -3.252 & 1.768 & -1.364 \\
\hline HETATM & 50 & C & 0 & -4.051 & 0.589 & 0.235 \\
\hline HETATM & 51 & C & 0 & -3.284 & -0.204 & 1.001 \\
\hline HETATM & 52 & $\mathrm{H}$ & 0 & -3.706 & -1.136 & 1.378 \\
\hline HETATM & 53 & 0 & 0 & -1.989 & 3.62 & 0.339 \\
\hline HETATM & 54 & $\mathrm{H}$ & 0 & -4.248 & 2.69 & -0.256 \\
\hline HETATM & 55 & C & 0 & -5.461 & 0.238 & -0.157 \\
\hline HETATM & 56 & $\mathrm{H}$ & 0 & -5.778 & -0.717 & 0.276 \\
\hline HETATM & 57 & $\mathrm{H}$ & 0 & -6.171 & 1.011 & 0.172 \\
\hline HETATM & 58 & $\mathrm{H}$ & 0 & -5.566 & 0.166 & -1.249 \\
\hline \multicolumn{7}{|l|}{ END } \\
\hline CONECT & 1 & 2 & 3 & 4 & 5 & \\
\hline CONECT & 2 & 1 & 6 & 9 & & \\
\hline CONECT & 3 & 1 & 14 & 17 & & \\
\hline CONECT & 4 & 1 & 10 & 13 & & \\
\hline CONECT & 5 & 1 & & & & \\
\hline CONECT & 6 & 2 & 7 & & & \\
\hline CONECT & 7 & 6 & 8 & 18 & & \\
\hline CONECT & 8 & 7 & 9 & 19 & & \\
\hline CONECT & 9 & 8 & 2 & 20 & & \\
\hline CONECT & 10 & 4 & 11 & 21 & & \\
\hline CONECT & 11 & 10 & 12 & 22 & & \\
\hline CONECT & 12 & 11 & 13 & 23 & & \\
\hline CONECT & 13 & 4 & 12 & & & \\
\hline CONECT & 14 & 3 & 15 & 24 & & \\
\hline CONECT & 15 & 14 & 16 & 25 & & \\
\hline CONECT & 16 & 15 & 17 & 26 & & \\
\hline CONECT & 17 & 3 & 16 & & & \\
\hline CONECT & 18 & 7 & & & & \\
\hline CONECT & 19 & 8 & & & & \\
\hline CONECT & 20 & 9 & & & & \\
\hline CONECT & 21 & 10 & & & & \\
\hline CONECT & 22 & 11 & & & & \\
\hline CONECT & 23 & 12 & & & & \\
\hline CONECT & 24 & 14 & & & & \\
\hline CONECT & 25 & 15 & & & & \\
\hline CONECT & 26 & 16 & & & & \\
\hline CONECT & 27 & 41 & & & & \\
\hline
\end{tabular}




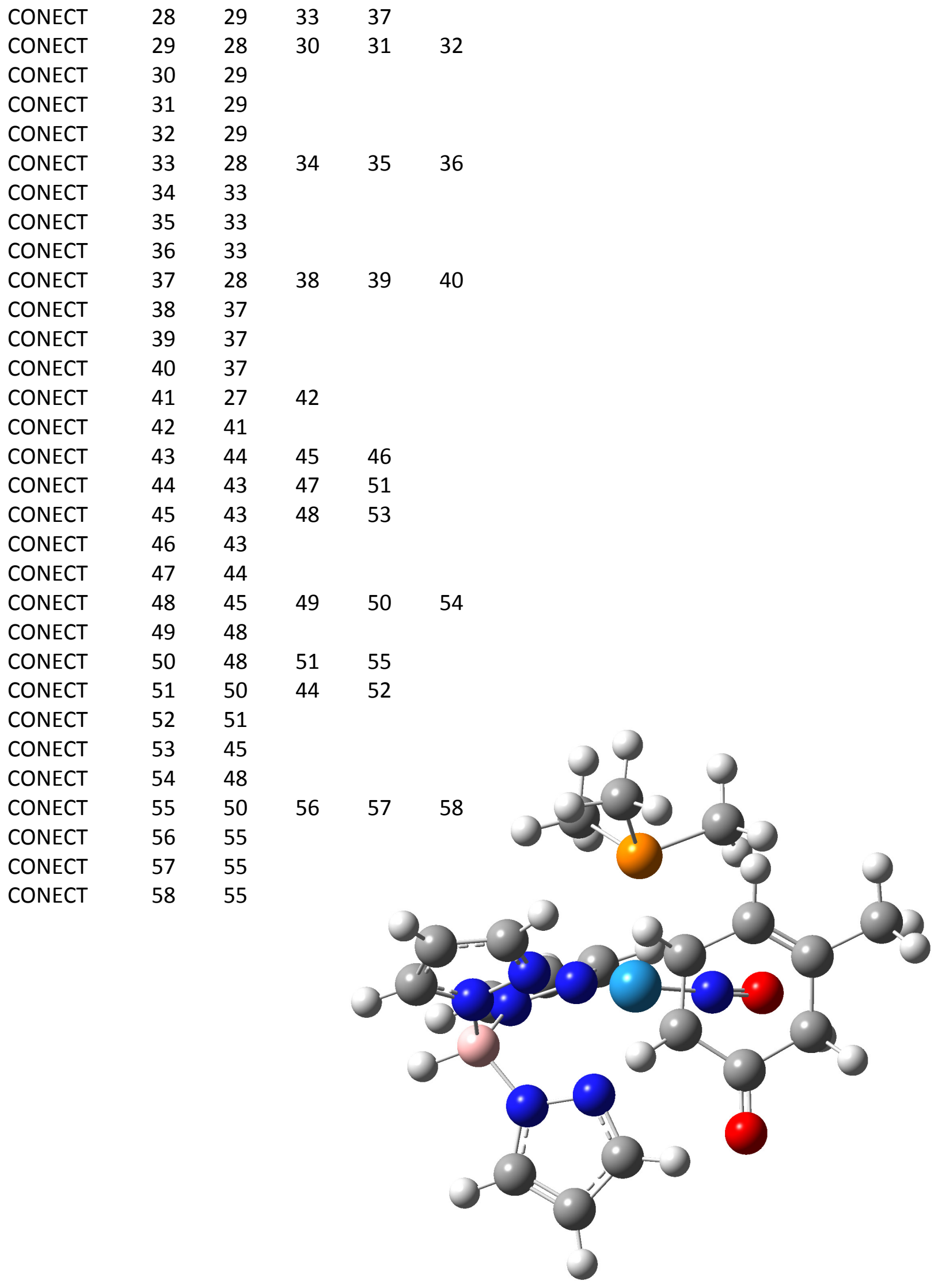




\begin{tabular}{|c|c|c|c|c|c|c|c|}
\hline TITLE & Exo & & & & & & \\
\hline REMARK & 1 & File & eat & y Gauss & View $5 . C$ & 0.8 & \\
\hline HETATM & 1 & $B$ & 0 & 1.561 & 2.62 & 0.368 & B \\
\hline HETATM & 2 & $N$ & 0 & 2.308 & 1.805 & -0.719 & 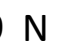 \\
\hline HETATM & 3 & $N$ & 0 & 0.117 & 2.823 & -0.168 & \\
\hline HETATM & 4 & $\mathrm{~N}$ & 0 & 1.512 & 1.827 & 1.685 & $\mathrm{~N}$ \\
\hline HETATM & 5 & $\mathrm{H}$ & 0 & 2.095 & 3.677 & 0.539 & $\mathrm{H}$ \\
\hline HETATM & 6 & $N$ & 0 & 1.779 & 0.63 & -1.169 & $\mathrm{~N}$ \\
\hline HETATM & 7 & C & 0 & 2.401 & 0.336 & -2.323 & $C$ \\
\hline HETATM & 8 & C & 0 & 3.358 & 1.312 & -2.621 & C \\
\hline HETATM & 9 & C & 0 & 3.255 & 2.229 & -1.584 & C \\
\hline HETATM & 10 & C & 0 & 1.954 & 2.191 & 2.906 & C \\
\hline HETATM & 11 & C & 0 & 1.652 & 1.178 & 3.806 & C \\
\hline HETATM & 12 & C & 0 & 1.008 & 0.207 & 3.034 & C \\
\hline HETATM & 13 & $\mathrm{~N}$ & 0 & 0.924 & 0.599 & 1.749 & $\mathrm{~N}$ \\
\hline HETATM & 14 & C & 0 & -0.459 & 3.957 & -0.622 & $C$ \\
\hline HETATM & 15 & C & 0 & -1.618 & 3.626 & -1.31 & C \\
\hline HETATM & 16 & C & 0 & -1.676 & 2.229 & -1.244 & C \\
\hline HETATM & 17 & $N$ & 0 & -0.637 & 1.749 & -0.543 & $\mathrm{~N}$ \\
\hline HETATM & 18 & $\mathrm{H}$ & 0 & 2.125 & -0.549 & -2.878 & $\mathrm{H}$ \\
\hline HETATM & 19 & $\mathrm{H}$ & 0 & 4.019 & 1.352 & -3.474 & $\mathrm{H}$ \\
\hline HETATM & 20 & $\mathrm{H}$ & 0 & 3.776 & 3.159 & -1.408 & $\mathrm{H}$ \\
\hline HETATM & 21 & $\mathrm{H}$ & 0 & 2.449 & 3.141 & 3.048 & $\mathrm{H}$ \\
\hline HETATM & 22 & $\mathrm{H}$ & 0 & 1.866 & 1.149 & 4.864 & $\mathrm{H}$ \\
\hline HETATM & 23 & $\mathrm{H}$ & 0 & 0.599 & -0.742 & 3.346 & $\mathrm{H}$ \\
\hline HETATM & 24 & $\mathrm{H}$ & 0 & 0.004 & 4.916 & -0.436 & $5 \mathrm{H}$ \\
\hline HETATM & 25 & $\mathrm{H}$ & 0 & -2.306 & 4.297 & -1.801 & $\mathrm{LH}$ \\
\hline HETATM & 26 & $\mathrm{H}$ & 0 & -2.399 & 1.55 & -1.671 & $\mathrm{LH}$ \\
\hline HETATM & 27 & W & 0 & 0.059 & -0.329 & -0.142 & W \\
\hline HETATM & 28 & $P$ & 0 & 1.805 & -2.223 & 0.127 & $P$ \\
\hline HETATM & 29 & C & 0 & 1.796 & -3.253 & 1.664 & C \\
\hline HETATM & 30 & $\mathrm{H}$ & 0 & 0.85 & -3.782 & 1.788 & $\mathrm{H}$ \\
\hline HETATM & 31 & $\mathrm{H}$ & 0 & 1.964 & -2.617 & 2.538 & $\mathrm{H}$ \\
\hline HETATM & 32 & $\mathrm{H}$ & 0 & 2.604 & -3.99 & 1.614 & $\mathrm{H}$ \\
\hline HETATM & 33 & C & 0 & 1.71 & -3.488 & -1.211 & L \\
\hline HETATM & 34 & $\mathrm{H}$ & 0 & 1.867 & -3.016 & -2.185 & $\mathrm{H}$ \\
\hline HETATM & 35 & $\mathrm{H}$ & 0 & 0.713 & -3.937 & -1.216 & $5 \mathrm{H}$ \\
\hline HETATM & 36 & $\mathrm{H}$ & 0 & 2.463 & -4.27 & -1.068 & $3 \mathrm{H}$ \\
\hline HETATM & 37 & C & 0 & 3.576 & -1.695 & 0.119 & C \\
\hline HETATM & 38 & $\mathrm{H}$ & 0 & 3.843 & -1.217 & -0.825 & $\mathrm{H}$ \\
\hline HETATM & 39 & $\mathrm{H}$ & 0 & 4.225 & -2.562 & 0.278 & $\mathrm{H}$ \\
\hline HETATM & 40 & $\mathrm{H}$ & 0 & 3.742 & -0.975 & 0.927 & $\mathrm{H}$ \\
\hline HETATM & 41 & $N$ & 0 & -0.579 & -0.99 & -1.671 & $\mathrm{~N}$ \\
\hline HETATM & 42 & 0 & 0 & -1.021 & -1.415 & -2.704 & \\
\hline
\end{tabular}




\begin{tabular}{|c|c|c|c|c|c|c|}
\hline HETATM & 43 & C & 0 & -1.868 & -0.558 & 1.097 \\
\hline HETATM & 44 & C & 0 & -1.054 & -1.778 & 1.148 \\
\hline HETATM & 45 & C & 0 & -3.07 & -0.517 & 0.339 \\
\hline HETATM & 46 & $\mathrm{H}$ & 0 & -1.773 & 0.139 & 1.921 \\
\hline HETATM & 47 & $\mathrm{H}$ & 0 & -0.596 & -1.956 & 2.119 \\
\hline HETATM & 48 & C & 0 & -3.431 & -1.667 & -0.581 \\
\hline HETATM & 49 & $\mathrm{H}$ & 0 & -3.224 & -1.382 & -1.627 \\
\hline HETATM & 50 & C & 0 & -2.702 & -2.947 & -0.268 \\
\hline HETATM & 51 & $\mathrm{H}$ & 0 & -3.093 & -3.845 & -0.737 \\
\hline HETATM & 52 & C & 0 & -1.627 & -2.989 & 0.525 \\
\hline HETATM & 53 & $\mathrm{H}$ & 0 & -1.149 & -3.945 & 0.727 \\
\hline HETATM & 54 & $\mathrm{H}$ & 0 & -4.512 & -1.842 & -0.527 \\
\hline HETATM & 55 & $\mathrm{~N}$ & 0 & -3.931 & 0.501 & 0.448 \\
\hline HETATM & 56 & C & 0 & -3.754 & 1.538 & 1.478 \\
\hline HETATM & 57 & $\mathrm{H}$ & 0 & -4.615 & 2.204 & 1.455 \\
\hline HETATM & 58 & $\mathrm{H}$ & 0 & -3.696 & 1.083 & 2.472 \\
\hline HETATM & 59 & $\mathrm{H}$ & 0 & -2.85 & 2.123 & 1.292 \\
\hline HETATM & 60 & C & 0 & -5.142 & 0.612 & -0.378 \\
\hline HETATM & 61 & $\mathrm{H}$ & 0 & -4.992 & 0.156 & -1.356 \\
\hline HETATM & 62 & $\mathrm{H}$ & 0 & -6.001 & 0.139 & 0.113 \\
\hline HETATM & 63 & $\mathrm{H}$ & 0 & -5.366 & 1.669 & -0.529 \\
\hline \multicolumn{7}{|l|}{ END } \\
\hline CONECT & 1 & 2 & 3 & 4 & 5 & \\
\hline CONECT & 2 & 1 & 6 & 9 & & \\
\hline CONECT & 3 & 1 & 14 & 17 & & \\
\hline CONECT & 4 & 1 & 10 & 13 & & \\
\hline CONECT & 5 & 1 & & & & \\
\hline CONECT & 6 & 2 & 7 & & & \\
\hline CONECT & 7 & 6 & 8 & 18 & & \\
\hline CONECT & 8 & 7 & 9 & 19 & & \\
\hline CONECT & 9 & 2 & 8 & 20 & & \\
\hline CONECT & 10 & 4 & 11 & 21 & & \\
\hline CONECT & 11 & 10 & 12 & 22 & & \\
\hline CONECT & 12 & 11 & 13 & 23 & & \\
\hline CONECT & 13 & 4 & 12 & & & \\
\hline CONECT & 14 & 3 & 15 & 24 & & \\
\hline CONECT & 15 & 14 & 16 & 25 & & \\
\hline CONECT & 16 & 15 & 17 & 26 & & \\
\hline CONECT & 17 & 16 & 3 & & & \\
\hline CONECT & 18 & 7 & & & & \\
\hline CONECT & 19 & 8 & & & & \\
\hline CONECT & 20 & 9 & & & & \\
\hline CONECT & 21 & 10 & & & & \\
\hline CONECT & 22 & 11 & & & & \\
\hline
\end{tabular}




\begin{tabular}{|c|c|c|c|c|c|}
\hline CONECT & 23 & 12 & & & \\
\hline CONECT & 24 & 14 & & & \\
\hline CONECT & 25 & 15 & & & \\
\hline CONECT & 26 & 16 & & & \\
\hline CONECT & 27 & 41 & & & \\
\hline CONECT & 28 & 29 & 33 & 37 & \\
\hline CONECT & 29 & 28 & 30 & 31 & 32 \\
\hline CONECT & 30 & 29 & & & \\
\hline CONECT & 31 & 29 & & & \\
\hline CONECT & 32 & 29 & & & \\
\hline CONECT & 33 & 28 & 34 & 35 & 36 \\
\hline CONECT & 34 & 33 & & & \\
\hline CONECT & 35 & 33 & & & \\
\hline CONECT & 36 & 33 & & & \\
\hline CONECT & 37 & 28 & 38 & 39 & 40 \\
\hline CONECT & 38 & 37 & & & \\
\hline CONECT & 39 & 37 & & & \\
\hline CONECT & 40 & 37 & & & \\
\hline CONECT & 41 & 27 & 42 & & \\
\hline CONECT & 42 & 41 & & & \\
\hline CONECT & 43 & 44 & 45 & 46 & \\
\hline CONECT & 44 & 43 & 47 & 52 & \\
\hline CONECT & 45 & 43 & 48 & 55 & \\
\hline CONECT & 46 & 43 & & & \\
\hline CONECT & 47 & 44 & & & \\
\hline CONECT & 48 & 45 & 49 & 50 & 54 \\
\hline CONECT & 49 & 48 & & & \\
\hline CONECT & 50 & 48 & 51 & 52 & \\
\hline CONECT & 51 & 50 & & & \\
\hline CONECT & 52 & 44 & 50 & 53 & \\
\hline CONECT & 53 & 52 & & & \\
\hline CONECT & 54 & 48 & & & \\
\hline CONECT & 55 & 45 & 56 & 60 & \\
\hline CONECT & 56 & 55 & 57 & 58 & 59 \\
\hline CONECT & 57 & 56 & & & \\
\hline CONECT & 58 & 56 & & & \\
\hline CONECT & 59 & 56 & & & \\
\hline CONECT & 60 & 55 & 61 & 62 & 63 \\
\hline CONECT & 61 & 60 & & & \\
\hline CONECT & 62 & 60 & & & \\
\hline CONECT & 63 & 60 & & & \\
\hline
\end{tabular}




\begin{tabular}{|c|c|c|c|c|c|c|c|}
\hline TITLE & 18 & & & & & & \\
\hline REMARK & 1 & & eat & y Gauss & View 5. & 0.8 & \\
\hline HETATM & 1 & $B$ & 0 & 3.032 & 0.219 & 0.496 & B \\
\hline HETATM & 2 & $N$ & 0 & 2.721 & -0.722 & -0.703 & $\mathrm{~N}$ \\
\hline HETATM & 3 & $N$ & 0 & 2.487 & 1.612 & 0.08 & $N$ \\
\hline HETATM & 4 & $\mathrm{~N}$ & 0 & 2.292 & -0.266 & 1.75 & $N$ \\
\hline HETATM & 5 & $\mathrm{H}$ & 0 & 4.21 & 0.268 & 0.694 & $\mathrm{H}$ \\
\hline HETATM & 6 & $N$ & 0 & 1.451 & -0.793 & -1.2 & $\mathrm{~N}$ \\
\hline HETATM & 7 & C & 0 & 1.536 & -1.333 & -2.429 & C \\
\hline HETATM & 8 & C & 0 & 2.866 & -1.637 & -2.731 & C \\
\hline HETATM & 9 & C & 0 & 3.582 & -1.221 & -1.616 & C \\
\hline HETATM & 10 & C & 0 & 2.79 & -0.595 & 2.958 & C \\
\hline HETATM & 11 & C & 0 & 1.734 & -0.922 & 3.798 & C \\
\hline HETATM & 12 & C & 0 & 0.595 & -0.762 & 3.006 & C \\
\hline HETATM & 13 & $\mathrm{~N}$ & 0 & 0.933 & -0.365 & 1.762 & $\mathrm{~N}$ \\
\hline HETATM & 14 & C & 0 & 3.191 & 2.706 & -0.28 & C \\
\hline HETATM & 15 & C & 0 & 2.349 & 3.584 & -0.948 & C \\
\hline HETATM & 16 & C & 0 & 1.114 & 2.933 & -0.976 & \\
\hline HETATM & 17 & $\mathrm{~N}$ & 0 & 1.193 & 1.75 & -0.34 & $\mathrm{~N}$ \\
\hline HETATM & 18 & $\mathrm{H}$ & 0 & 0.647 & -1.47 & -3.027 & \\
\hline HETATM & 19 & $\mathrm{H}$ & 0 & 3.253 & -2.082 & -3.635 & \\
\hline HETATM & 20 & $\mathrm{H}$ & 0 & 4.643 & -1.228 & -1.415 & $\mathrm{H}$ \\
\hline HETATM & 21 & $\mathrm{H}$ & 0 & 3.856 & -0.57 & 3.137 & $\mathrm{H}$ \\
\hline HETATM & 22 & $\mathrm{H}$ & 0 & 1.783 & -1.228 & 4.832 & \\
\hline HETATM & 23 & $\mathrm{H}$ & 0 & -0.44 & -0.907 & 3.276 & $\mathrm{H}$ \\
\hline HETATM & 24 & $\mathrm{H}$ & 0 & 4.244 & 2.777 & -0.046 & $\mathrm{H}$ \\
\hline HETATM & 25 & $\mathrm{H}$ & 0 & 2.597 & 4.55 & -1.363 & \\
\hline HETATM & 26 & $\mathrm{H}$ & 0 & 0.185 & 3.247 & -1.428 & $\mathrm{H}$ \\
\hline HETATM & 27 & W & 0 & -0.246 & 0.089 & -0.118 & \\
\hline HETATM & 28 & $P$ & 0 & -0.849 & -2.474 & -0.105 & \\
\hline HETATM & 29 & C & 0 & -1.801 & -3.169 & 1.313 & C \\
\hline HETATM & 30 & $\mathrm{H}$ & 0 & -2.798 & -2.725 & 1.332 & $\mathrm{H}$ \\
\hline HETATM & 31 & $\mathrm{H}$ & 0 & -1.28 & -2.981 & 2.256 & $\mathrm{H}$ \\
\hline HETATM & 32 & $\mathrm{H}$ & 0 & -1.889 & -4.253 & 1.178 & $\mathrm{H}$ \\
\hline HETATM & 33 & C & 0 & -1.844 & -2.992 & -1.564 & C \\
\hline HETATM & 34 & $\mathrm{H}$ & 0 & -1.343 & -2.716 & -2.496 & $\Pi$ \\
\hline HETATM & 35 & $\mathrm{H}$ & 0 & -2.813 & -2.488 & -1.517 & $\mathrm{H}$ \\
\hline HETATM & 36 & $\mathrm{H}$ & 0 & -2 & -4.076 & -1.551 & \\
\hline HETATM & 37 & C & 0 & 0.624 & -3.589 & -0.108 & $C$ \\
\hline HETATM & 38 & $\mathrm{H}$ & 0 & 1.213 & -3.481 & -1.019 & $\mathrm{H}$ \\
\hline HETATM & 39 & $\mathrm{H}$ & 0 & 0.287 & -4.628 & -0.026 & \\
\hline HETATM & 40 & $\mathrm{H}$ & 0 & 1.261 & -3.358 & 0.751 & $\mathrm{H}$ \\
\hline HETATM & 41 & $\mathrm{~N}$ & 0 & -1.095 & 0.406 & -1.658 & \\
\hline HETATM & 42 & 0 & 0 & -1.635 & 0.629 & -2.701 & \\
\hline
\end{tabular}




\begin{tabular}{|c|c|c|c|c|c|c|}
\hline HETATM & 43 & C & 0 & -1.912 & 2.513 & 0.633 \\
\hline HETATM & 44 & C & 0 & -1.564 & 1.414 & 1.391 \\
\hline HETATM & 45 & $C$ & 0 & -3.001 & 2.443 & -0.405 \\
\hline HETATM & 46 & $\mathrm{H}$ & 0 & -2.554 & 2.436 & -1.409 \\
\hline HETATM & 47 & $\mathrm{H}$ & 0 & -0.918 & 1.568 & 2.252 \\
\hline HETATM & 48 & $C$ & 0 & -3.954 & 1.247 & -0.242 \\
\hline HETATM & 49 & $\mathrm{H}$ & 0 & -4.442 & 0.999 & -1.188 \\
\hline HETATM & 50 & $C$ & 0 & -3.357 & -0.031 & 0.335 \\
\hline HETATM & 51 & $C$ & 0 & -2.099 & 0.079 & 1.13 \\
\hline HETATM & 52 & $\mathrm{H}$ & 0 & -2.084 & -0.6 & 1.978 \\
\hline HETATM & 53 & $\mathrm{O}$ & 0 & -3.937 & -1.099 & 0.191 \\
\hline HETATM & 54 & $\mathrm{H}$ & 0 & -4.759 & 1.516 & 0.457 \\
\hline HETATM & 55 & $\mathrm{H}$ & 0 & -3.57 & 3.381 & -0.36 \\
\hline HETATM & 56 & $C$ & 0 & -1.358 & 3.869 & 0.948 \\
\hline HETATM & 57 & $\mathrm{H}$ & 0 & -2.1 & 4.413 & 1.552 \\
\hline HETATM & 58 & $\mathrm{H}$ & 0 & -0.426 & 3.824 & 1.516 \\
\hline HETATM & 59 & $\mathrm{H}$ & 0 & -1.204 & 4.469 & 0.044 \\
\hline \multicolumn{7}{|l|}{ END } \\
\hline CONECT & 1 & 2 & 3 & 4 & 5 & \\
\hline CONECT & 2 & 1 & 6 & 9 & & \\
\hline CONECT & 3 & 1 & 14 & 17 & & \\
\hline CONECT & 4 & 1 & 10 & 13 & & \\
\hline CONECT & 5 & 1 & & & & \\
\hline CONECT & 6 & 2 & 7 & & & \\
\hline CONECT & 7 & 6 & 8 & 18 & & \\
\hline CONECT & 8 & 7 & 9 & 19 & & \\
\hline CONECT & 9 & 8 & 2 & 20 & & \\
\hline CONECT & 10 & 4 & 11 & 21 & & \\
\hline CONECT & 11 & 10 & 12 & 22 & & \\
\hline CONECT & 12 & 11 & 13 & 23 & & \\
\hline CONECT & 13 & 4 & 12 & & & \\
\hline CONECT & 14 & 3 & 15 & 24 & & \\
\hline CONECT & 15 & 14 & 16 & 25 & & \\
\hline CONECT & 16 & 15 & 17 & 26 & & \\
\hline CONECT & 17 & 16 & 3 & & & \\
\hline CONECT & 18 & 7 & & & & \\
\hline CONECT & 19 & 8 & & & & \\
\hline CONECT & 20 & 9 & & & & \\
\hline CONECT & 21 & 10 & & & & \\
\hline CONECT & 22 & 11 & & & & \\
\hline CONECT & 23 & 12 & & & & \\
\hline CONECT & 24 & 14 & & & & \\
\hline CONECT & 25 & 15 & & & & \\
\hline CONECT & 26 & 16 & & & & \\
\hline
\end{tabular}




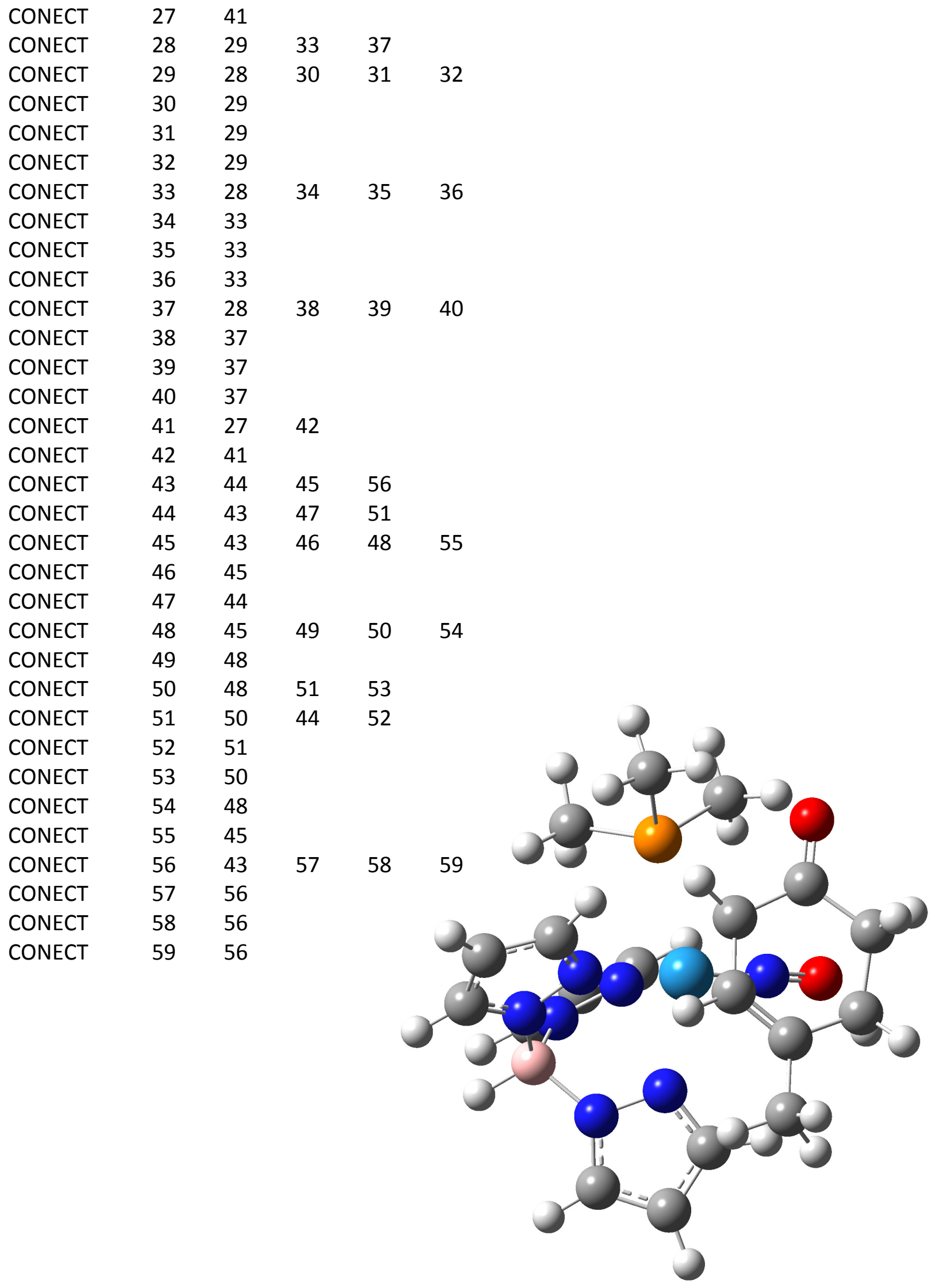




\begin{tabular}{|c|c|c|c|c|c|c|c|}
\hline TITLE & $20 p$ & & & & & & \\
\hline REMARK & 1 & & eat & y Gauss & View 5. & 0.8 & \\
\hline HETATM & 1 & $B$ & 0 & 2.93 & 0.589 & 0.274 & \\
\hline HETATM & 2 & $\mathrm{~N}$ & 0 & 2.578 & -0.171 & -1.034 & \\
\hline HETATM & 3 & $\mathrm{~N}$ & 0 & 2.177 & 1.927 & 0.189 & \\
\hline HETATM & 4 & $\mathrm{~N}$ & 0 & 2.421 & -0.216 & 1.486 & \\
\hline HETATM & 5 & $\mathrm{H}$ & 0 & 4.111 & 0.758 & 0.358 & $\mathrm{H}$ \\
\hline HETATM & 6 & $\mathrm{~N}$ & 0 & 1.267 & -0.303 & -1.382 & $\mathrm{~N}$ \\
\hline HETATM & 7 & C & 0 & 1.233 & -0.605 & -2.695 & C \\
\hline HETATM & 8 & C & 0 & 2.532 & -0.696 & -3.199 & C \\
\hline HETATM & 9 & C & 0 & 3.35 & -0.398 & -2.115 & C \\
\hline HETATM & 10 & C & 0 & 3.125 & -0.723 & 2.517 & C \\
\hline HETATM & 11 & C & 0 & 2.248 & -1.369 & 3.38 & C \\
\hline HETATM & 12 & C & 0 & 0.995 & -1.202 & 2.789 & C \\
\hline HETATM & 13 & $\mathrm{~N}$ & 0 & 1.097 & -0.5 & 1.641 & $\mathrm{~N}$ \\
\hline HETATM & 14 & C & 0 & 2.681 & 3.175 & 0.08 & C \\
\hline HETATM & 15 & C & 0 & 1.644 & 4.053 & -0.197 & C \\
\hline HETATM & 16 & C & 0 & 0.5 & 3.252 & -0.254 & C \\
\hline HETATM & 17 & $\mathrm{~N}$ & 0 & 0.824 & 1.967 & -0.016 & $\mathrm{~N}$ \\
\hline HETATM & 18 & $\mathrm{H}$ & 0 & 0.289 & -0.722 & -3.206 & $\mathrm{H}$ \\
\hline HETATM & 19 & $\mathrm{H}$ & 0 & 2.833 & -0.925 & -4.21 & $\mathrm{H}$ \\
\hline HETATM & 20 & $\mathrm{H}$ & 0 & 4.425 & -0.314 & -2.043 & $\mathrm{H}$ \\
\hline HETATM & 21 & $\mathrm{H}$ & 0 & 4.195 & -0.585 & 2.568 & $\mathrm{H}$ \\
\hline HETATM & 22 & $\mathrm{H}$ & 0 & 2.484 & -1.874 & 4.305 & $\mathrm{H}$ \\
\hline HETATM & 23 & $\mathrm{H}$ & 0 & 0.035 & -1.549 & 3.143 & $\mathrm{H}$ \\
\hline HETATM & 24 & $\mathrm{H}$ & 0 & 3.74 & 3.345 & 0.206 & $\mathrm{H}$ \\
\hline HETATM & 25 & $\mathrm{H}$ & 0 & 1.704 & 5.121 & -0.338 & $\mathrm{H}$ \\
\hline HETATM & 26 & $\mathrm{H}$ & 0 & -0.531 & 3.536 & -0.422 & $\mathrm{H}$ \\
\hline HETATM & 27 & W & 0 & -0.377 & 0.083 & 0.018 & \\
\hline HETATM & 28 & $P$ & 0 & -0.563 & -2.526 & -0.316 & $\mathrm{P}$ \\
\hline HETATM & 29 & C & 0 & -1.306 & -3.656 & 0.95 & C \\
\hline HETATM & 30 & $\mathrm{H}$ & 0 & -2.395 & -3.566 & 0.972 & $\mathrm{H}$ \\
\hline HETATM & 31 & $\mathrm{H}$ & 0 & -0.908 & -3.448 & 1.947 & $\mathrm{H}$ \\
\hline HETATM & 32 & $\mathrm{H}$ & 0 & -1.057 & -4.689 & 0.689 & $\mathrm{H}$ \\
\hline HETATM & 33 & C & 0 & -1.494 & -3 & -1.835 & C \\
\hline HETATM & 34 & $\mathrm{H}$ & 0 & -1.035 & -2.558 & -2.722 & $\mathrm{H}$ \\
\hline HETATM & 35 & $\mathrm{H}$ & 0 & -2.524 & -2.638 & -1.77 & $\mathrm{H}$ \\
\hline HETATM & 36 & $\mathrm{H}$ & 0 & -1.504 & -4.089 & -1.949 & $\mathrm{H}$ \\
\hline HETATM & 37 & C & 0 & 1.093 & -3.317 & -0.527 & C \\
\hline HETATM & 38 & $\mathrm{H}$ & 0 & 1.622 & -2.903 & -1.386 & $\mathrm{H}$ \\
\hline HETATM & 39 & $\mathrm{H}$ & 0 & 0.963 & -4.394 & -0.675 & $\mathrm{H}$ \\
\hline HETATM & 40 & $\mathrm{H}$ & 0 & 1.695 & -3.154 & 0.371 & $\mathrm{H}$ \\
\hline HETATM & 41 & $\mathrm{~N}$ & 0 & -1.399 & 0.36 & -1.426 & $\mathrm{~N}$ \\
\hline HETATM & 42 & 0 & 0 & -2.028 & 0.448 & -2.433 & \\
\hline
\end{tabular}




\begin{tabular}{|c|c|c|c|c|c|c|}
\hline HETATM & 43 & C & 0 & -1.834 & 0.371 & 1.882 \\
\hline HETATM & 44 & C & 0 & -2.552 & -0.617 & 1.209 \\
\hline HETATM & 45 & C & 0 & -1.671 & 1.627 & 1.255 \\
\hline HETATM & 46 & $\mathrm{H}$ & 0 & -1.072 & 2.383 & 1.749 \\
\hline HETATM & 47 & $\mathrm{H}$ & 0 & -1.287 & 0.143 & 2.791 \\
\hline HETATM & 48 & $\mathrm{H}$ & 0 & -2.574 & -1.599 & 1.666 \\
\hline HETATM & 49 & C & 0 & -2.743 & 2.136 & 0.344 \\
\hline HETATM & 50 & C & 0 & -3.972 & 1.246 & 0.182 \\
\hline HETATM & 51 & $\mathrm{H}$ & 0 & -4.452 & 1.499 & -0.766 \\
\hline HETATM & 52 & C & 0 & -3.721 & -0.273 & 0.3 \\
\hline HETATM & 53 & $\mathrm{H}$ & 0 & -3.603 & -0.713 & -0.695 \\
\hline HETATM & 54 & $\mathrm{H}$ & 0 & -4.61 & -0.752 & 0.731 \\
\hline HETATM & 55 & $\mathrm{H}$ & 0 & -4.657 & 1.564 & 0.98 \\
\hline HETATM & 56 & 0 & 0 & -2.675 & 3.237 & -0.176 \\
\hline \multicolumn{7}{|l|}{ END } \\
\hline CONECT & 1 & 2 & 3 & 4 & 5 & \\
\hline CONECT & 2 & 1 & 6 & 9 & & \\
\hline CONECT & 3 & 1 & 14 & 17 & & \\
\hline CONECT & 4 & 1 & 10 & 13 & & \\
\hline CONECT & 5 & 1 & & & & \\
\hline CONECT & 6 & 2 & 7 & & & \\
\hline CONECT & 7 & 6 & 8 & 18 & & \\
\hline CONECT & 8 & 7 & 9 & 19 & & \\
\hline CONECT & 9 & 8 & 2 & 20 & & \\
\hline CONECT & 10 & 4 & 11 & 21 & & \\
\hline CONECT & 11 & 10 & 12 & 22 & & \\
\hline CONECT & 12 & 11 & 13 & 23 & & \\
\hline CONECT & 13 & 4 & 12 & & & \\
\hline CONECT & 14 & 3 & 15 & 24 & & \\
\hline CONECT & 15 & 14 & 16 & 25 & & \\
\hline CONECT & 16 & 15 & 17 & 26 & & \\
\hline CONECT & 17 & 3 & 16 & & & \\
\hline CONECT & 18 & 7 & & & & \\
\hline CONECT & 19 & 8 & & & & \\
\hline CONECT & 20 & 9 & & & & \\
\hline CONECT & 21 & 10 & & & & \\
\hline CONECT & 22 & 11 & & & & \\
\hline CONECT & 23 & 12 & & & & \\
\hline CONECT & 24 & 14 & & & & \\
\hline CONECT & 25 & 15 & & & & \\
\hline CONECT & 26 & 16 & & & & \\
\hline CONECT & 27 & 41 & & & & \\
\hline CONECT & 28 & 29 & 33 & 37 & & \\
\hline CONECT & 29 & 28 & 30 & 31 & 32 & \\
\hline
\end{tabular}




$\begin{array}{llllll}\text { CONECT } & 30 & 29 & & & \\ \text { CONECT } & 31 & 29 & & & \\ \text { CONECT } & 32 & 29 & & & \\ \text { CONECT } & 33 & 28 & 34 & 35 & 36 \\ \text { CONECT } & 34 & 33 & & & \\ \text { CONECT } & 35 & 33 & & & \\ \text { CONECT } & 36 & 33 & & & \\ \text { CONECT } & 37 & 28 & 38 & 39 & 40 \\ \text { CONECT } & 38 & 37 & & & \\ \text { CONECT } & 39 & 37 & & & \\ \text { CONECT } & 40 & 37 & & & \\ \text { CONECT } & 41 & 27 & 42 & & \\ \text { CONECT } & 42 & 41 & & & \\ \text { CONECT } & 43 & 44 & 45 & 47 & \\ \text { CONECT } & 44 & 43 & 48 & 52 & \\ \text { CONECT } & 45 & 43 & 46 & 49 & \\ \text { CONECT } & 46 & 45 & & & \\ \text { CONECT } & 47 & 43 & & & \\ \text { CONECT } & 48 & 44 & & & \\ \text { CONECT } & 49 & 45 & 50 & 56 & \\ \text { CONECT } & 50 & 49 & 51 & 52 & 55 \\ \text { CONECT } & 51 & 50 & & & \\ \text { CONECT } & 52 & 44 & 50 & 53 & 54 \\ \text { CONECT } & 53 & 52 & & & \\ \text { CONECT } & 54 & 52 & & & \\ \text { CONECT } & 55 & 50 & & & \\ \text { CONECT } & 56 & 49 & & & \end{array}$
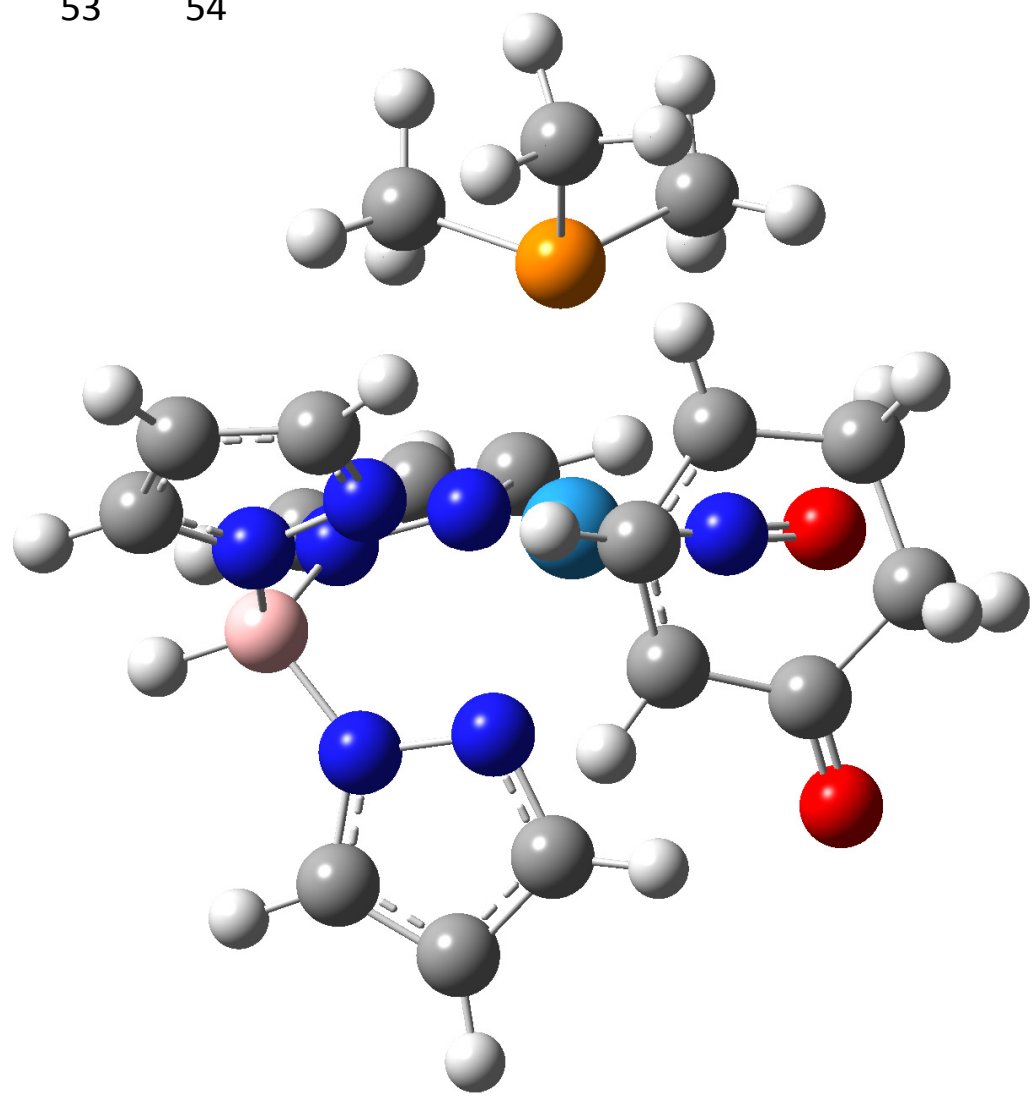


\begin{tabular}{|c|c|c|c|c|c|c|c|}
\hline TITLE & 20d & & & & & & \\
\hline REMARK & 1 & & $d t$ & y Gauss & View 5. & 0.8 & \\
\hline HETATM & 1 & B & 0 & 3.014 & -0.252 & 0.413 & $B$ \\
\hline HETATM & 2 & $N$ & 0 & 2.512 & -0.915 & -0.899 & $N$ \\
\hline HETATM & 3 & $\mathrm{~N}$ & 0 & 2.717 & 1.261 & 0.251 & $\mathrm{~N}$ \\
\hline HETATM & 4 & $N$ & 0 & 2.229 & -0.801 & 1.612 & $\mathrm{~N}$ \\
\hline HETATM & 5 & $\mathrm{H}$ & 0 & 4.186 & -0.441 & 0.556 & $\mathrm{H}$ \\
\hline HETATM & 6 & $N$ & 0 & 1.237 & -0.68 & -1.324 & $\mathrm{~N}$ \\
\hline HETATM & 7 & C & 0 & 1.185 & -1.016 & -2.627 & C \\
\hline HETATM & 8 & C & 0 & 2.427 & -1.495 & -3.049 & C \\
\hline HETATM & 9 & C & 0 & 3.24 & -1.4 & -1.926 & C \\
\hline HETATM & 10 & C & 0 & 2.691 & -1.399 & 2.729 & C \\
\hline HETATM & 11 & C & 0 & 1.616 & -1.66 & 3.568 & C \\
\hline HETATM & 12 & C & 0 & 0.505 & -1.177 & 2.874 & C \\
\hline HETATM & 13 & $\mathrm{~N}$ & 0 & 0.876 & -0.656 & 1.687 & $\mathrm{~N}$ \\
\hline HETATM & 14 & C & 0 & 3.598 & 2.267 & 0.076 & C \\
\hline HETATM & 15 & C & 0 & 2.914 & 3.394 & -0.358 & C \\
\hline HETATM & 16 & C & 0 & 1.582 & 2.985 & -0.44 & C \\
\hline HETATM & 17 & $\mathrm{~N}$ & 0 & 1.459 & 1.699 & -0.059 & \\
\hline HETATM & 18 & $\mathrm{H}$ & 0 & 0.27 & -0.891 & -3.187 & $\mathrm{H}$ \\
\hline HETATM & 19 & $\mathrm{H}$ & 0 & 2.699 & -1.845 & -4.034 & \\
\hline HETATM & 20 & $\mathrm{H}$ & 0 & 4.287 & -1.628 & -1.791 & $\mathrm{H}$ \\
\hline HETATM & 21 & $\mathrm{H}$ & 0 & 3.747 & -1.591 & 2.849 & $\mathrm{H}$ \\
\hline HETATM & 22 & $\mathrm{H}$ & 0 & 1.636 & -2.128 & 4.541 & $\mathrm{H}$ \\
\hline HETATM & 23 & $\mathrm{H}$ & 0 & -0.531 & -1.173 & 3.179 & $\mathrm{H}$ \\
\hline HETATM & 24 & $\mathrm{H}$ & 0 & 4.65 & 2.108 & 0.264 & $\mathrm{H}$ \\
\hline HETATM & 25 & $\mathrm{H}$ & 0 & 3.322 & 4.367 & -0.588 & $\mathrm{H}$ \\
\hline HETATM & 26 & $\mathrm{H}$ & 0 & 0.719 & 3.545 & -0.765 & $\mathrm{H}$ \\
\hline HETATM & 27 & W & 0 & -0.26 & 0.276 & -0.037 & \\
\hline HETATM & 28 & $P$ & 0 & -1.268 & -2.136 & -0.356 & $\mathrm{P}$ \\
\hline HETATM & 29 & C & 0 & -2.307 & -2.88 & 0.973 & C \\
\hline HETATM & 30 & $\mathrm{H}$ & 0 & -3.229 & -2.306 & 1.079 & $\mathrm{H}$ \\
\hline HETATM & 31 & $\mathrm{H}$ & 0 & -1.761 & -2.903 & 1.921 & $\mathrm{H}$ \\
\hline HETATM & 32 & $\mathrm{H}$ & 0 & -2.552 & -3.91 & 0.691 & $\mathrm{H}$ \\
\hline HETATM & 33 & C & 0 & -2.34 & -2.284 & -1.844 & $C$ \\
\hline HETATM & 34 & $\mathrm{H}$ & 0 & -1.806 & -1.973 & -2.745 & $\mathrm{H}$ \\
\hline HETATM & 35 & $\mathrm{H}$ & 0 & -3.216 & -1.643 & -1.708 & $\mathrm{H}$ \\
\hline HETATM & 36 & $\mathrm{H}$ & 0 & -2.67 & -3.321 & -1.966 & $\mathrm{H}$ \\
\hline HETATM & 37 & C & 0 & 0.022 & -3.442 & -0.56 & $C$ \\
\hline HETATM & 38 & $\mathrm{H}$ & 0 & 0.618 & -3.285 & -1.46 & $\mathrm{H}$ \\
\hline HETATM & 39 & $\mathrm{H}$ & 0 & -0.467 & -4.419 & -0.626 & $\mathrm{H}$ \\
\hline HETATM & 40 & $\mathrm{H}$ & 0 & 0.688 & -3.44 & 0.308 & $\mathrm{H}$ \\
\hline HETATM & 41 & $\mathrm{~N}$ & 0 & -1.083 & 0.887 & -1.505 & $\mathrm{~N}$ \\
\hline HETATM & 42 & $\mathrm{O}$ & 0 & -1.601 & 1.267 & -2.509 & \\
\hline
\end{tabular}




\begin{tabular}{|c|c|c|c|c|c|c|}
\hline HETATM & 43 & C & 0 & -1.319 & 2.598 & 0.914 \\
\hline HETATM & 44 & C & 0 & -1.303 & 1.467 & 1.708 \\
\hline HETATM & 45 & C & 0 & -2.474 & 2.978 & 0.032 \\
\hline HETATM & 46 & $\mathrm{H}$ & 0 & -2.157 & 3.095 & -1.011 \\
\hline HETATM & 47 & $\mathrm{H}$ & 0 & -0.575 & 3.364 & 1.112 \\
\hline HETATM & 48 & $\mathrm{H}$ & 0 & -0.622 & 1.408 & 2.551 \\
\hline HETATM & 49 & C & 0 & -3.669 & 2.008 & 0.133 \\
\hline HETATM & 50 & $\mathrm{H}$ & 0 & -4.248 & 1.99 & -0.794 \\
\hline HETATM & 51 & C & 0 & -3.341 & 0.569 & 0.517 \\
\hline HETATM & 52 & C & 0 & -2.104 & 0.332 & 1.32 \\
\hline HETATM & 53 & $\mathrm{H}$ & 0 & -2.195 & -0.482 & 2.03 \\
\hline HETATM & 54 & 0 & 0 & -4.105 & -0.343 & 0.232 \\
\hline HETATM & 55 & $\mathrm{H}$ & 0 & -4.354 & 2.354 & 0.92 \\
\hline HETATM & 56 & $\mathrm{H}$ & 0 & -2.786 & 3.983 & 0.348 \\
\hline \multicolumn{7}{|l|}{ END } \\
\hline CONECT & 1 & 2 & 3 & 4 & 5 & \\
\hline CONECT & 2 & 1 & 6 & 9 & & \\
\hline CONECT & 3 & 1 & 14 & 17 & & \\
\hline CONECT & 4 & 1 & 10 & 13 & & \\
\hline CONECT & 5 & 1 & & & & \\
\hline CONECT & 6 & 2 & 7 & & & \\
\hline CONECT & 7 & 6 & 8 & 18 & & \\
\hline CONECT & 8 & 7 & 9 & 19 & & \\
\hline CONECT & 9 & 8 & 2 & 20 & & \\
\hline CONECT & 10 & 4 & 11 & 21 & & \\
\hline CONECT & 11 & 10 & 12 & 22 & & \\
\hline CONECT & 12 & 11 & 13 & 23 & & \\
\hline CONECT & 13 & 4 & 12 & & & \\
\hline CONECT & 14 & 3 & 15 & 24 & & \\
\hline CONECT & 15 & 14 & 16 & 25 & & \\
\hline CONECT & 16 & 15 & 17 & 26 & & \\
\hline CONECT & 17 & 3 & 16 & & & \\
\hline CONECT & 18 & 7 & & & & \\
\hline CONECT & 19 & 8 & & & & \\
\hline CONECT & 20 & 9 & & & & \\
\hline CONECT & 21 & 10 & & & & \\
\hline CONECT & 22 & 11 & & & & \\
\hline CONECT & 23 & 12 & & & & \\
\hline CONECT & 24 & 14 & & & & \\
\hline CONECT & 25 & 15 & & & & \\
\hline CONECT & 26 & 16 & & & & \\
\hline CONECT & 27 & 41 & & & & \\
\hline CONECT & 28 & 29 & 33 & 37 & & \\
\hline CONECT & 29 & 28 & 30 & 31 & 32 & \\
\hline
\end{tabular}




$\begin{array}{llllll}\text { CONECT } & 30 & 29 & & & \\ \text { CONECT } & 31 & 29 & & & \\ \text { CONECT } & 32 & 29 & & & \\ \text { CONECT } & 33 & 28 & 34 & 35 & 36 \\ \text { CONECT } & 34 & 33 & & & \\ \text { CONECT } & 35 & 33 & & & \\ \text { CONECT } & 36 & 33 & & & \\ \text { CONECT } & 37 & 28 & 38 & 39 & 40 \\ \text { CONECT } & 38 & 37 & & & \\ \text { CONECT } & 39 & 37 & & & \\ \text { CONECT } & 40 & 37 & & & \\ \text { CONECT } & 41 & 27 & 42 & & \\ \text { CONECT } & 42 & 41 & & & \\ \text { CONECT } & 43 & 44 & 45 & 47 & \\ \text { CONECT } & 44 & 43 & 48 & 52 & \\ \text { CONECT } & 45 & 43 & 46 & 49 & 56 \\ \text { CONECT } & 46 & 45 & & & \\ \text { CONECT } & 47 & 43 & & & \\ \text { CONECT } & 48 & 44 & & & \\ \text { CONECT } & 49 & 45 & 50 & 51 & 55 \\ \text { CONECT } & 50 & 49 & & & \\ \text { CONECT } & 51 & 49 & 52 & 54 & \\ \text { CONECT } & 52 & 51 & 44 & 53 & \\ \text { CONECT } & 53 & 52 & & & \\ \text { CONECT } & 54 & 51 & & & \\ \text { CONECT } & 55 & 49 & & & \\ \text { CONECT } & 56 & 45 & & & \end{array}$

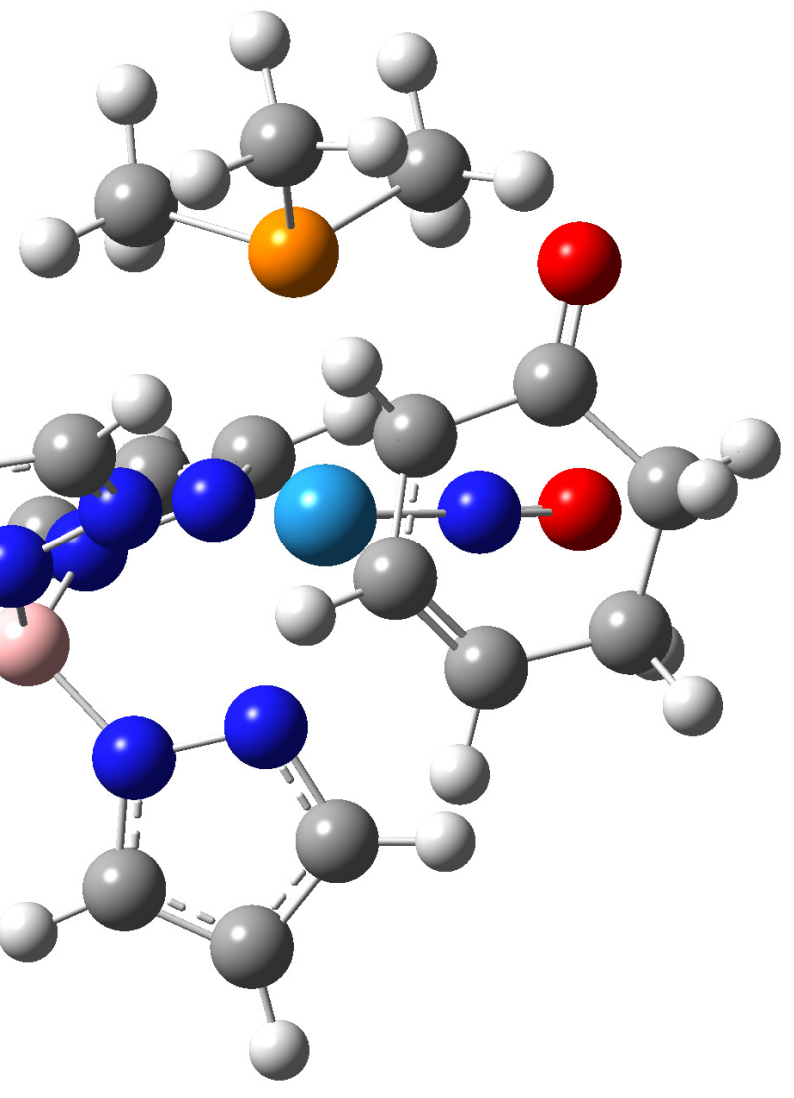




\begin{tabular}{|c|c|c|c|c|c|c|c|}
\hline TITLE & 21d' & & & & & & \\
\hline REMARK & 1 & File & ate & / Gauss I & View 5.0 & 0.8 & \\
\hline HETATM & 1 & W & 0 & -0.048 & -0.168 & 0.003 & \\
\hline HETATM & 2 & $P$ & 0 & 1.265 & -2.451 & -0.366 & $P$ \\
\hline HETATM & 3 & C & 0 & 0.823 & -3.286 & -1.949 & $C$ \\
\hline HETATM & 4 & $\mathrm{H}$ & 0 & 1.354 & -4.24 & -2.02 & $\mathrm{H}$ \\
\hline HETATM & 5 & $\mathrm{H}$ & 0 & -0.254 & -3.475 & -1.996 & $\mathrm{H}$ \\
\hline HETATM & 6 & $\mathrm{H}$ & 0 & 1.102 & -2.673 & -2.808 & $\mathrm{H}$ \\
\hline HETATM & 7 & C & 0 & 1.168 & -3.847 & 0.842 & C \\
\hline HETATM & 8 & $\mathrm{H}$ & 0 & 1.852 & -4.633 & 0.507 & $\mathrm{H}$ \\
\hline HETATM & 9 & $\mathrm{H}$ & 0 & 1.482 & -3.529 & 1.839 & $\mathrm{H}$ \\
\hline HETATM & 10 & $\mathrm{H}$ & 0 & 0.162 & -4.272 & 0.894 & $\mathrm{H}$ \\
\hline HETATM & 11 & C & 0 & 3.087 & -2.193 & -0.454 & C \\
\hline HETATM & 12 & $\mathrm{H}$ & 0 & 3.576 & -3.161 & -0.607 & $\mathrm{H}$ \\
\hline HETATM & 13 & $\mathrm{H}$ & 0 & 3.355 & -1.529 & -1.277 & $\mathrm{H}$ \\
\hline HETATM & 14 & $\mathrm{H}$ & 0 & 3.447 & -1.762 & 0.484 & $\mathrm{H}$ \\
\hline HETATM & 15 & $N$ & 0 & -1.054 & -0.561 & -1.432 & $\mathrm{~N}$ \\
\hline HETATM & 16 & $\mathrm{O}$ & 0 & -1.632 & -0.878 & -2.424 & $\mathrm{O}$ \\
\hline HETATM & 17 & C & 0 & -1.206 & -1.804 & 1.447 & C \\
\hline HETATM & 18 & C & 0 & -1.4 & -0.508 & 1.928 & C \\
\hline HETATM & 19 & $N$ & 0 & 2.53 & 1.279 & -0.994 & $\mathrm{~N}$ \\
\hline HETATM & 20 & $N$ & 0 & 1.541 & 0.406 & -1.357 & $\mathrm{~N}$ \\
\hline HETATM & 21 & $N$ & 0 & 2.403 & 1.151 & 1.516 & $\mathrm{~N}$ \\
\hline HETATM & 22 & $N$ & 0 & 1.481 & 0.153 & 1.624 & $\mathrm{~N}$ \\
\hline HETATM & 23 & C & 0 & 3.307 & 1.536 & -2.064 & C \\
\hline HETATM & 24 & $\mathrm{H}$ & 0 & 4.136 & 2.224 & -1.983 & $\mathrm{H}$ \\
\hline HETATM & 25 & C & 0 & 1.713 & 0.141 & -2.673 & C \\
\hline HETATM & 26 & $\mathrm{H}$ & 0 & 1.022 & -0.501 & -3.198 & $\mathrm{H}$ \\
\hline HETATM & 27 & C & 0 & 3.25 & 1.112 & 2.563 & C \\
\hline HETATM & 28 & $\mathrm{H}$ & 0 & 4.048 & 1.835 & 2.65 & $\mathrm{H}$ \\
\hline HETATM & 29 & C & 0 & 1.777 & -0.512 & 2.764 & C \\
\hline HETATM & 30 & $\mathrm{H}$ & 0 & 1.184 & -1.356 & 3.086 & $\mathrm{H}$ \\
\hline HETATM & 31 & C & 0 & 2.828 & 0.818 & -3.157 & C \\
\hline HETATM & 32 & $\mathrm{H}$ & 0 & 3.225 & 0.806 & -4.161 & $\mathrm{H}$ \\
\hline HETATM & 33 & $B$ & 0 & 2.383 & 2.103 & 0.316 & B \\
\hline HETATM & 34 & $\mathrm{H}$ & 0 & 3.249 & 2.919 & 0.403 & $\mathrm{H}$ \\
\hline HETATM & 35 & C & 0 & 2.884 & 0.056 & 3.391 & C \\
\hline HETATM & 36 & $\mathrm{H}$ & 0 & 3.35 & -0.247 & 4.317 & $\mathrm{H}$ \\
\hline HETATM & 37 & $N$ & 0 & -0.13 & 2.041 & -0.051 & $\mathrm{~N}$ \\
\hline HETATM & 38 & $N$ & 0 & 0.991 & 2.781 & 0.209 & N \\
\hline HETATM & 39 & C & 0 & -1.087 & 2.911 & -0.434 & C \\
\hline HETATM & 40 & $\mathrm{H}$ & 0 & -2.06 & 2.551 & -0.729 & $\mathrm{H}$ \\
\hline HETATM & 41 & C & 0 & 0.721 & 4.086 & 0.007 & C \\
\hline HETATM & 42 & $\mathrm{H}$ & 0 & 1.489 & 4.832 & 0.154 & \\
\hline
\end{tabular}




\begin{tabular}{|c|c|c|c|c|c|c|}
\hline HETATM & 43 & $C$ & 0 & -0.603 & 4.217 & -0.397 \\
\hline HETATM & 44 & $\mathrm{H}$ & 0 & -1.129 & 5.128 & -0.64 \\
\hline HETATM & 45 & C & 0 & -2.235 & 0.381 & 1.216 \\
\hline HETATM & 46 & C & 0 & -3.359 & -0.137 & 0.458 \\
\hline HETATM & 47 & $\mathrm{H}$ & 0 & -0.558 & -2.444 & 2.033 \\
\hline HETATM & 48 & $C$ & 0 & -2.337 & -2.521 & 0.734 \\
\hline HETATM & 49 & $\mathrm{H}$ & 0 & -2.823 & -3.157 & 1.487 \\
\hline HETATM & 50 & $\mathrm{H}$ & 0 & -1.955 & -3.206 & -0.028 \\
\hline HETATM & 51 & $C$ & 0 & -3.406 & -1.603 & 0.088 \\
\hline HETATM & 52 & $\mathrm{H}$ & 0 & -3.329 & -1.664 & -1.005 \\
\hline HETATM & 53 & $\mathrm{H}$ & 0 & -4.401 & -1.986 & 0.335 \\
\hline HETATM & 54 & $\mathrm{H}$ & 0 & -0.826 & -0.113 & 2.759 \\
\hline HETATM & 55 & $\mathrm{H}$ & 0 & -2.244 & 1.411 & 1.539 \\
\hline HETATM & 56 & $\mathrm{~N}$ & 0 & -4.379 & 0.621 & 0.097 \\
\hline HETATM & 57 & C & 0 & -5.508 & 0.091 & -0.702 \\
\hline HETATM & 58 & $\mathrm{H}$ & 0 & -5.997 & 0.93 & -1.198 \\
\hline HETATM & 59 & $\mathrm{H}$ & 0 & -5.158 & -0.606 & -1.462 \\
\hline HETATM & 60 & $\mathrm{H}$ & 0 & -6.233 & -0.404 & -0.047 \\
\hline HETATM & 61 & C & 0 & -4.589 & 2.027 & 0.501 \\
\hline HETATM & 62 & $\mathrm{H}$ & 0 & -4.504 & 2.676 & -0.375 \\
\hline HETATM & 63 & $\mathrm{H}$ & 0 & -5.603 & 2.111 & 0.901 \\
\hline HETATM & 64 & $\mathrm{H}$ & 0 & -3.889 & 2.343 & 1.269 \\
\hline \multicolumn{7}{|l|}{ END } \\
\hline CONECT & 1 & 15 & & & & \\
\hline CONECT & 2 & 3 & 7 & 11 & & \\
\hline CONECT & 3 & 2 & 4 & 5 & 6 & \\
\hline CONECT & 4 & 3 & & & & \\
\hline CONECT & 5 & 3 & & & & \\
\hline CONECT & 6 & 3 & & & & \\
\hline CONECT & 7 & 2 & 8 & 9 & 10 & \\
\hline CONECT & 8 & 7 & & & & \\
\hline CONECT & 9 & 7 & & & & \\
\hline CONECT & 10 & 7 & & & & \\
\hline CONECT & 11 & 2 & 12 & 13 & 14 & \\
\hline CONECT & 12 & 11 & & & & \\
\hline CONECT & 13 & 11 & & & & \\
\hline CONECT & 14 & 11 & & & & \\
\hline CONECT & 15 & 1 & 16 & & & \\
\hline CONECT & 16 & 15 & & & & \\
\hline CONECT & 17 & 18 & 47 & 48 & & \\
\hline CONECT & 18 & 17 & 45 & 54 & & \\
\hline CONECT & 19 & 20 & 23 & 33 & & \\
\hline CONECT & 20 & 19 & 25 & & & \\
\hline CONECT & 21 & 22 & 27 & 33 & & \\
\hline
\end{tabular}




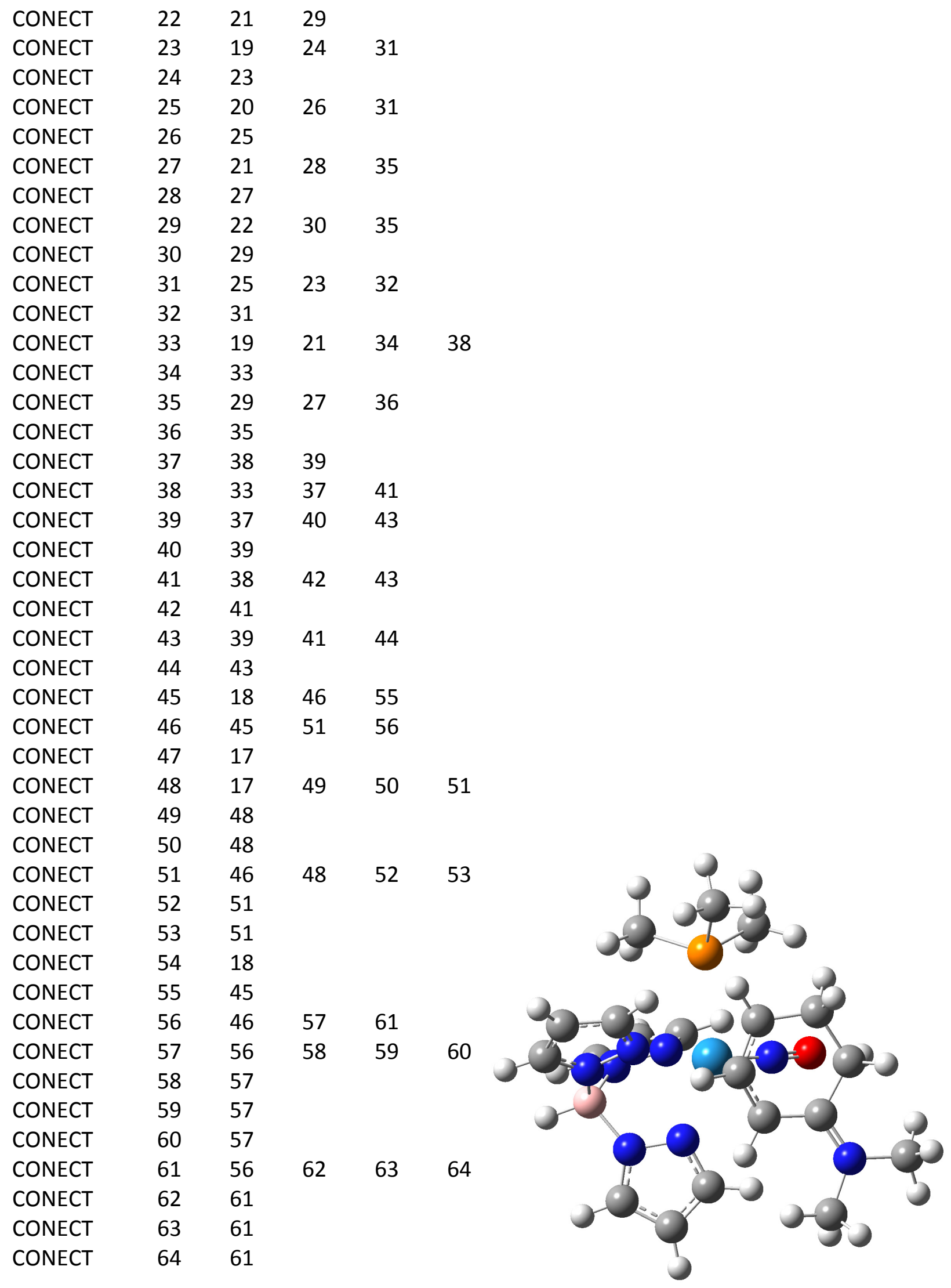




$\begin{array}{llllllll}\text { TITLE } & 21 d & & & & & & \\ \text { REMARK } & 1 & \text { File created by } & \text { Gauss View } & 5.0 .8 & \\ \text { HETATM } & 1 & \mathrm{~B} & 0 & 1.338 & 2.702 & 0.305 & \mathrm{~B} \\ \text { HETATM } & 2 & \mathrm{~N} & 0 & 2.09 & 1.922 & -0.805 & \mathrm{~N} \\ \text { HETATM } & 3 & \mathrm{~N} & 0 & -0.138 & 2.771 & -0.171 & \mathrm{~N} \\ \text { HETATM } & 4 & \mathrm{~N} & 0 & 1.404 & 1.916 & 1.622 & \mathrm{~N} \\ \text { HETATM } & 5 & \mathrm{H} & 0 & 1.794 & 3.795 & 0.442 & \mathrm{H} \\ \text { HETATM } & 6 & \mathrm{~N} & 0 & 1.605 & 0.715 & -1.221 & \mathrm{~N} \\ \text { HETATM } & 7 & \mathrm{C} & 0 & 2.173 & 0.449 & -2.417 & \mathrm{C} \\ \text { HETATM } & 8 & \mathrm{C} & 0 & 3.052 & 1.473 & -2.767 & \mathrm{C} \\ \text { HETATM } & 9 & \mathrm{C} & 0 & 2.956 & 2.391 & -1.726 & \mathrm{C} \\ \text { HETATM } & 10 & \mathrm{C} & 0 & 1.882 & 2.322 & 2.814 & \mathrm{C} \\ \text { HETATM } & 11 & \mathrm{C} & 0 & 1.713 & 1.296 & 3.735 & \mathrm{C} \\ \text { HETATM } & 12 & \mathrm{C} & 0 & 1.11 & 0.273 & 3.009 & \mathrm{C} \\ \text { HETATM } & 13 & \mathrm{~N} & 0 & 0.917 & 0.641 & 1.72 & \mathrm{~N} \\ \text { HETATM } & 14 & \mathrm{C} & 0 & -0.835 & 3.839 & -0.61 & \mathrm{C} \\ \text { HETATM } & 15 & \mathrm{C} & 0 & -1.977 & 3.394 & -1.264 & \mathrm{C} \\ \text { HETATM } & 16 & \mathrm{C} & 0 & -1.899 & 2.002 & -1.2 & \mathrm{C} \\ \text { HETATM } & 17 & \mathrm{~N} & 0 & -0.796 & 1.628 & -0.523 & \mathrm{~N} \\ \text { HETATM } & 40 & \mathrm{H} & 0 & 3.709 & -0.316 & 1.071 & \mathrm{H} \\ \text { HETATM } & 41 & \mathrm{~N} & 0 & -0.538 & -1.017 & -1.682 & \mathrm{~N} \\ \text { HETATM } & 42 & \mathrm{O} & 0 & -0.925 & -1.397 & -2.738 & \mathrm{O}\end{array}$




\begin{tabular}{|c|c|c|c|c|c|c|}
\hline HETATM & 43 & C & 0 & -1.884 & -0.564 & 1.168 \\
\hline HETATM & 44 & C & 0 & -1.041 & -1.72 & 1.413 \\
\hline HETATM & 45 & C & 0 & -3.097 & -0.733 & 0.401 \\
\hline HETATM & 46 & $\mathrm{H}$ & 0 & -0.458 & -1.789 & 2.322 \\
\hline HETATM & 47 & C & 0 & -3.232 & -1.894 & -0.565 \\
\hline HETATM & 48 & $\mathrm{H}$ & 0 & -3.191 & -1.494 & -1.586 \\
\hline HETATM & 49 & $\mathrm{H}$ & 0 & -4.23 & -2.329 & -0.452 \\
\hline HETATM & 50 & $N$ & 0 & -4.098 & 0.121 & 0.517 \\
\hline HETATM & 51 & C & 0 & -4.109 & 1.203 & 1.528 \\
\hline HETATM & 52 & $\mathrm{H}$ & 0 & -3.805 & 0.817 & 2.503 \\
\hline HETATM & 53 & $\mathrm{H}$ & 0 & -3.45 & 2.02 & 1.222 \\
\hline HETATM & 54 & $\mathrm{H}$ & 0 & -5.128 & 1.579 & 1.612 \\
\hline HETATM & 55 & C & 0 & -5.32 & 0.065 & -0.313 \\
\hline HETATM & 56 & $\mathrm{H}$ & 0 & -5.163 & -0.509 & -1.223 \\
\hline HETATM & 57 & $\mathrm{H}$ & 0 & -6.14 & -0.375 & 0.264 \\
\hline HETATM & 58 & $\mathrm{H}$ & 0 & -5.594 & 1.086 & -0.589 \\
\hline HETATM & 59 & C & 0 & -1.038 & -2.751 & 0.493 \\
\hline HETATM & 60 & $\mathrm{H}$ & 0 & -0.342 & -3.573 & 0.626 \\
\hline HETATM & 61 & $C$ & 0 & -2.177 & -3.019 & -0.435 \\
\hline HETATM & 62 & $\mathrm{H}$ & 0 & -2.652 & -3.927 & -0.032 \\
\hline HETATM & 63 & $\mathrm{H}$ & 0 & -1.821 & -3.306 & -1.43 \\
\hline HETATM & 64 & $\mathrm{H}$ & 0 & -1.873 & 0.203 & 1.93 \\
\hline \multicolumn{7}{|l|}{ END } \\
\hline CONECT & 1 & 2 & 3 & 4 & 5 & \\
\hline CONECT & 2 & 1 & 6 & 9 & & \\
\hline CONECT & 3 & 1 & 14 & 17 & & \\
\hline CONECT & 4 & 1 & 10 & 13 & & \\
\hline CONECT & 5 & 1 & & & & \\
\hline CONECT & 6 & 2 & 7 & & & \\
\hline CONECT & 7 & 6 & 8 & 18 & & \\
\hline CONECT & 8 & 7 & 9 & 19 & & \\
\hline CONECT & 9 & 2 & 8 & 20 & & \\
\hline CONECT & 10 & 4 & 11 & 21 & & \\
\hline CONECT & 11 & 10 & 12 & 22 & & \\
\hline CONECT & 12 & 11 & 13 & 23 & & \\
\hline CONECT & 13 & 4 & 12 & & & \\
\hline CONECT & 14 & 3 & 15 & 24 & & \\
\hline CONECT & 15 & 14 & 16 & 25 & & \\
\hline CONECT & 16 & 15 & 17 & 26 & & \\
\hline CONECT & 17 & 16 & 3 & & & \\
\hline CONECT & 18 & 7 & & & & \\
\hline CONECT & 19 & 8 & & & & \\
\hline CONECT & 20 & 9 & & & & \\
\hline CONECT & 21 & 10 & & & & \\
\hline
\end{tabular}




\begin{tabular}{|c|c|c|c|c|c|}
\hline CONECT & 22 & 11 & & & \\
\hline CONECT & 23 & 12 & & & \\
\hline CONECT & 24 & 14 & & & \\
\hline CONECT & 25 & 15 & & & \\
\hline CONECT & 26 & 16 & & & \\
\hline CONECT & 27 & 41 & & & \\
\hline CONECT & 28 & 29 & 33 & 37 & \\
\hline CONECT & 29 & 28 & 30 & 31 & 32 \\
\hline CONECT & 30 & 29 & & & \\
\hline CONECT & 31 & 29 & & & \\
\hline CONECT & 32 & 29 & & & \\
\hline CONECT & 33 & 28 & 34 & 35 & 36 \\
\hline CONECT & 34 & 33 & & & \\
\hline CONECT & 35 & 33 & & & \\
\hline CONECT & 36 & 33 & & & \\
\hline CONECT & 37 & 28 & 38 & 39 & 40 \\
\hline CONECT & 38 & 37 & & & \\
\hline CONECT & 39 & 37 & & & \\
\hline CONECT & 40 & 37 & & & \\
\hline CONECT & 41 & 27 & 42 & & \\
\hline CONECT & 42 & 41 & & & \\
\hline CONECT & 43 & 44 & 45 & 64 & \\
\hline CONECT & 44 & 43 & 46 & 59 & \\
\hline CONECT & 45 & 43 & 47 & 50 & \\
\hline CONECT & 46 & 44 & & & \\
\hline CONECT & 47 & 45 & 48 & 49 & 61 \\
\hline CONECT & 48 & 47 & & & \\
\hline CONECT & 49 & 47 & & & \\
\hline CONECT & 50 & 45 & 51 & 55 & \\
\hline CONECT & 51 & 50 & 52 & 53 & 54 \\
\hline CONECT & 52 & 51 & & & \\
\hline CONECT & 53 & 51 & & & \\
\hline CONECT & 54 & 51 & & & \\
\hline CONECT & 55 & 50 & 56 & 57 & 58 \\
\hline CONECT & 56 & 55 & & & \\
\hline CONECT & 57 & 55 & & & \\
\hline CONECT & 58 & 55 & & & \\
\hline CONECT & 59 & 44 & 60 & 61 & \\
\hline CONECT & 60 & 59 & & & \\
\hline CONECT & 61 & 47 & 59 & 62 & 63 \\
\hline CONECT & 62 & 61 & & & \\
\hline CONECT & 63 & 61 & & & \\
\hline CONECT & 64 & 43 & & & \\
\hline
\end{tabular}


Theoretical Tungsten Species:

TITLE $\quad\left[\left(\mathrm{NH}_{3}\right)_{5} \mathbf{W}\left(\mathrm{C}_{3} \mathrm{H}_{5}\right)\right]^{+}$

\begin{tabular}{llllllll} 
REMARK & 1 & \multicolumn{7}{l}{ File created by Gauss View 5.0 .8} \\
HETATM & 1 & $\mathrm{~N}$ & 0 & 0.564 & 1.774 & -1.362 & $\mathrm{~N}$ \\
HETATM & 2 & $\mathrm{H}$ & 0 & 1.315 & 1.459 & -1.984 & $\mathrm{H}$ \\
HETATM & 3 & $\mathrm{H}$ & 0 & -0.161 & 2.13 & -1.989 & $\mathrm{H}$ \\
HETATM & 4 & $\mathrm{~N}$ & 0 & 0.52 & 1.486 & 1.672 & $\mathrm{~N}$ \\
HETATM & 5 & $\mathrm{H}$ & 0 & 1.266 & 1.058 & 2.232 & $\mathrm{H}$ \\
HETATM & 6 & $\mathrm{H}$ & 0 & 0.893 & 2.394 & 1.375 & $\mathrm{H}$ \\
HETATM & 7 & $\mathrm{~N}$ & 0 & -2.155 & 1.095 & -0.007 & $\mathrm{~N}$ \\
HETATM & 8 & $\mathrm{H}$ & 0 & -2.189 & 1.862 & -0.68 & $\mathrm{H}$ \\
HETATM & 9 & $\mathrm{H}$ & 0 & -2.967 & 0.507 & -0.22 & $\mathrm{H}$ \\
HETATM & 10 & $\mathrm{~N}$ & 0 & -1.236 & -1.271 & -1.539 & $\mathrm{~N}$ \\
HETATM & 11 & $\mathrm{H}$ & 0 & -0.493 & -1.835 & -1.949 & $\mathrm{H}$ \\
HETATM & 12 & $\mathrm{H}$ & 0 & -1.938 & -1.934 & -1.193 & $\mathrm{H}$ \\
HETATM & 13 & $\mathrm{~N}$ & 0 & -1.304 & -1.379 & 1.378 & $\mathrm{~N}$ \\
HETATM & 14 & $\mathrm{H}$ & 0 & -2.15 & -1.835 & 1.017 & $\mathrm{H}$ \\
HETATM & 15 & $\mathrm{H}$ & 0 & -0.63 & -2.12 & 1.565 & $\mathrm{H}$ \\
HETATM & 16 & $\mathrm{H}$ & 0 & -1.563 & -1.01 & 2.295 & $\mathrm{H}$ \\
HETATM & 17 & $\mathrm{H}$ & 0 & 0.938 & 2.601 & -0.885 & $\mathrm{H}$ \\
HETATM & 18 & $\mathrm{H}$ & 0 & -0.22 & 1.711 & 2.342 & $\mathrm{H}$ \\
HETATM & 19 & $\mathrm{H}$ & 0 & -2.363 & 1.526 & 0.896 & $\mathrm{H}$ \\
HETATM & 20 & $\mathrm{H}$ & 0 & -1.682 & -0.79 & -2.325 & $\mathrm{H}$ \\
HETATM & 21 & $\mathrm{C}$ & 0 & 2.082 & -0.367 & 0 & $\mathrm{C}$ \\
HETATM & 22 & $\mathrm{H}$ & 0 & 2.851 & 0.403 & 0.098 & $\mathrm{H}$ \\
HETATM & 23 & $\mathrm{C}$ & 0 & 1.57 & -0.861 & -1.255 & $\mathrm{C}$ \\
HETATM & 24 & $\mathrm{H}$ & 0 & 1.551 & -1.951 & -1.393 & $\mathrm{H}$ \\
HETATM & 25 & $\mathrm{H}$ & 0 & 1.91 & -0.363 & -2.174 & $\mathrm{H}$ \\
HETATM & 26 & $\mathrm{~W}$ & 0 & -0.047 & 0.017 & -0.003 & $\mathrm{~W}$ \\
HETATM & 27 & $\mathrm{C}$ & 0 & 1.519 & -1.11 & 1.103 & $\mathrm{C}$ \\
HETATM & 28 & $\mathrm{H}$ & 0 & 1.815 & -0.805 & 2.117 & $\mathrm{H}$ \\
HETATM & 29 & $\mathrm{H}$ & 0 & 1.514 & -2.205 & 1.016 & $\mathrm{H}$ \\
HENA & & & & & & &
\end{tabular}

END

$\begin{array}{lllll}\text { CONECT } & 1 & 2 & 3 & 17 \\ \text { CONECT } & 2 & 1 & & \\ \text { CONECT } & 3 & 1 & & \\ \text { CONECT } & 4 & 5 & 6 & 18 \\ \text { CONECT } & 5 & 4 & & \\ \text { CONECT } & 6 & 4 & & \\ \text { CONECT } & 7 & 8 & 9 & 19 \\ \text { CONECT } & 8 & 7 & & \\ \text { CONECT } & 9 & 7 & & \\ \text { CONECT } & 10 & 11 & 12 & 20 \\ \text { CONECT } & 11 & 10 & & \end{array}$



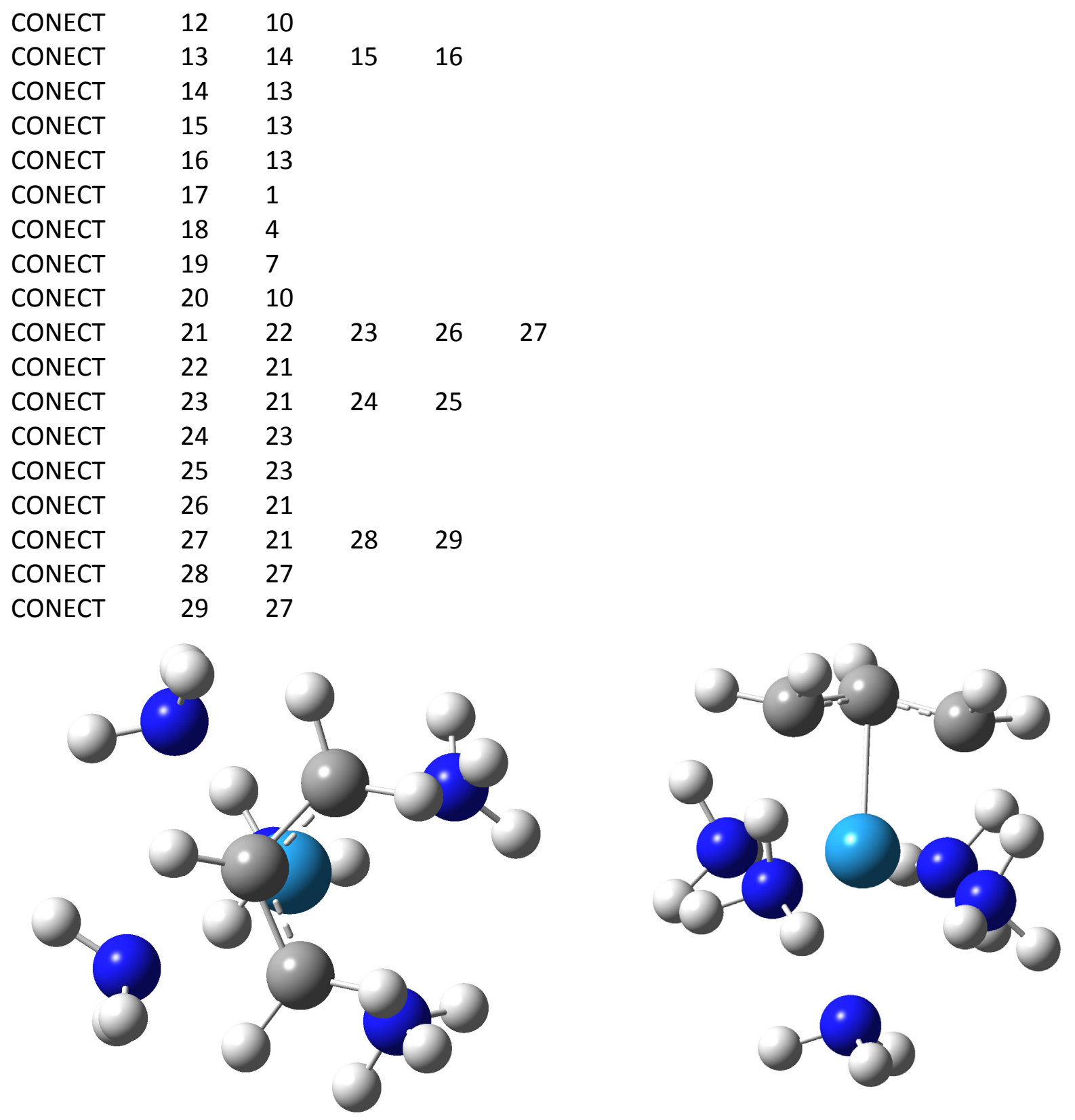


\begin{tabular}{|c|c|c|c|c|c|c|c|}
\hline TITLE & \multicolumn{7}{|c|}{$\left[\left(\mathrm{NH}_{3}\right)_{4} \mathrm{~W}(\mathrm{NO})\left(\mathrm{C}_{3} \mathrm{H}_{5}\right)\right]^{2+}$ Terminus 1} \\
\hline REMARK & 1 & \multicolumn{6}{|c|}{ File created by GaussView 5.0.8 } \\
\hline HETATM & 1 & W & 0 & 0.105 & -0.042 & 0.022 & W \\
\hline HETATM & 2 & $\mathrm{~N}$ & 0 & -0.125 & 1.738 & -0.058 & $\mathrm{~N}$ \\
\hline HETATM & 3 & $\mathrm{O}$ & 0 & -0.245 & 2.906 & -0.151 & $\mathrm{O}$ \\
\hline HETATM & 4 & $\mathrm{C}$ & 0 & -1.435 & -0.545 & -1.564 & C \\
\hline HETATM & 5 & $\mathrm{H}$ & 0 & -1.729 & 0.353 & -2.107 & $\mathrm{H}$ \\
\hline HETATM & 6 & $\mathrm{H}$ & 0 & -1.225 & -1.394 & -2.215 & $\mathrm{H}$ \\
\hline HETATM & 7 & $C$ & 0 & -2.101 & -0.823 & -0.33 & C \\
\hline HETATM & 8 & $\mathrm{H}$ & 0 & -2.231 & -1.847 & 0.016 & $\mathrm{H}$ \\
\hline HETATM & 9 & C & 0 & -2.398 & 0.225 & 0.527 & C \\
\hline HETATM & 10 & $\mathrm{H}$ & 0 & -2.773 & 0.032 & 1.529 & $\mathrm{H}$ \\
\hline HETATM & 11 & $\mathrm{~N}$ & 0 & 2.282 & 0.301 & 0.693 & $\mathrm{~N}$ \\
\hline HETATM & 12 & $\mathrm{H}$ & 0 & 2.933 & -0.49 & 0.678 & $\mathrm{H}$ \\
\hline HETATM & 13 & $\mathrm{H}$ & 0 & 2.733 & 1.039 & 0.142 & $\mathrm{H}$ \\
\hline HETATM & 14 & $\mathrm{~N}$ & 0 & 1.215 & 0.028 & -1.978 & $\mathrm{~N}$ \\
\hline HETATM & 15 & $\mathrm{H}$ & 0 & 2.214 & 0.252 & -1.948 & $\mathrm{H}$ \\
\hline HETATM & 16 & $\mathrm{H}$ & 0 & 1.144 & -0.821 & -2.545 & $\mathrm{H}$ \\
\hline HETATM & 17 & $\mathrm{~N}$ & 0 & 0.589 & -2.348 & 0.027 & $\mathrm{~N}$ \\
\hline HETATM & 18 & $\mathrm{H}$ & 0 & 1.568 & -2.607 & -0.127 & $\mathrm{H}$ \\
\hline HETATM & 19 & $\mathrm{H}$ & 0 & 0.326 & -2.848 & 0.881 & $\mathrm{H}$ \\
\hline HETATM & 20 & $\mathrm{~N}$ & 0 & -0.138 & -0.275 & 2.293 & $\mathrm{~N}$ \\
\hline HETATM & 21 & $\mathrm{H}$ & 0 & 0.709 & -0.55 & 2.799 & $\mathrm{H}$ \\
\hline HETATM & 22 & $\mathrm{H}$ & 0 & -0.438 & 0.605 & 2.722 & $\mathrm{H}$ \\
\hline HETATM & 23 & $\mathrm{H}$ & 0 & 0.797 & 0.764 & -2.554 & $\mathrm{H}$ \\
\hline HETATM & 24 & $\mathrm{H}$ & 0 & 0.078 & -2.835 & -0.717 & $\mathrm{H}$ \\
\hline HETATM & 25 & $\mathrm{H}$ & 0 & 2.329 & 0.666 & 1.649 & $\mathrm{H}$ \\
\hline HETATM & 26 & $\mathrm{H}$ & 0 & -0.844 & -0.96 & 2.578 & $\mathrm{H}$ \\
\hline HETATM & 27 & $\mathrm{H}$ & 0 & -2.546 & 1.234 & 0.155 & $\mathrm{H}$ \\
\hline \multicolumn{8}{|l|}{ END } \\
\hline CONECT & 1 & 2 & & & & & \\
\hline CONECT & 2 & 1 & 3 & & & & \\
\hline CONECT & 3 & 2 & & & & & \\
\hline CONECT & 4 & 5 & 6 & 7 & & & \\
\hline CONECT & 5 & 4 & & & & & \\
\hline CONECT & 6 & 4 & & & & & \\
\hline CONECT & 7 & 4 & 8 & 9 & & & \\
\hline CONECT & 8 & 7 & & & & & \\
\hline CONECT & 9 & 7 & 10 & 27 & & & \\
\hline CONECT & 10 & 9 & & & & & \\
\hline CONECT & 11 & 12 & 13 & 25 & & & \\
\hline CONECT & 12 & 11 & & & & & \\
\hline CONECT & 13 & 11 & & & & & \\
\hline CONECT & 14 & 15 & 16 & 23 & & & \\
\hline
\end{tabular}




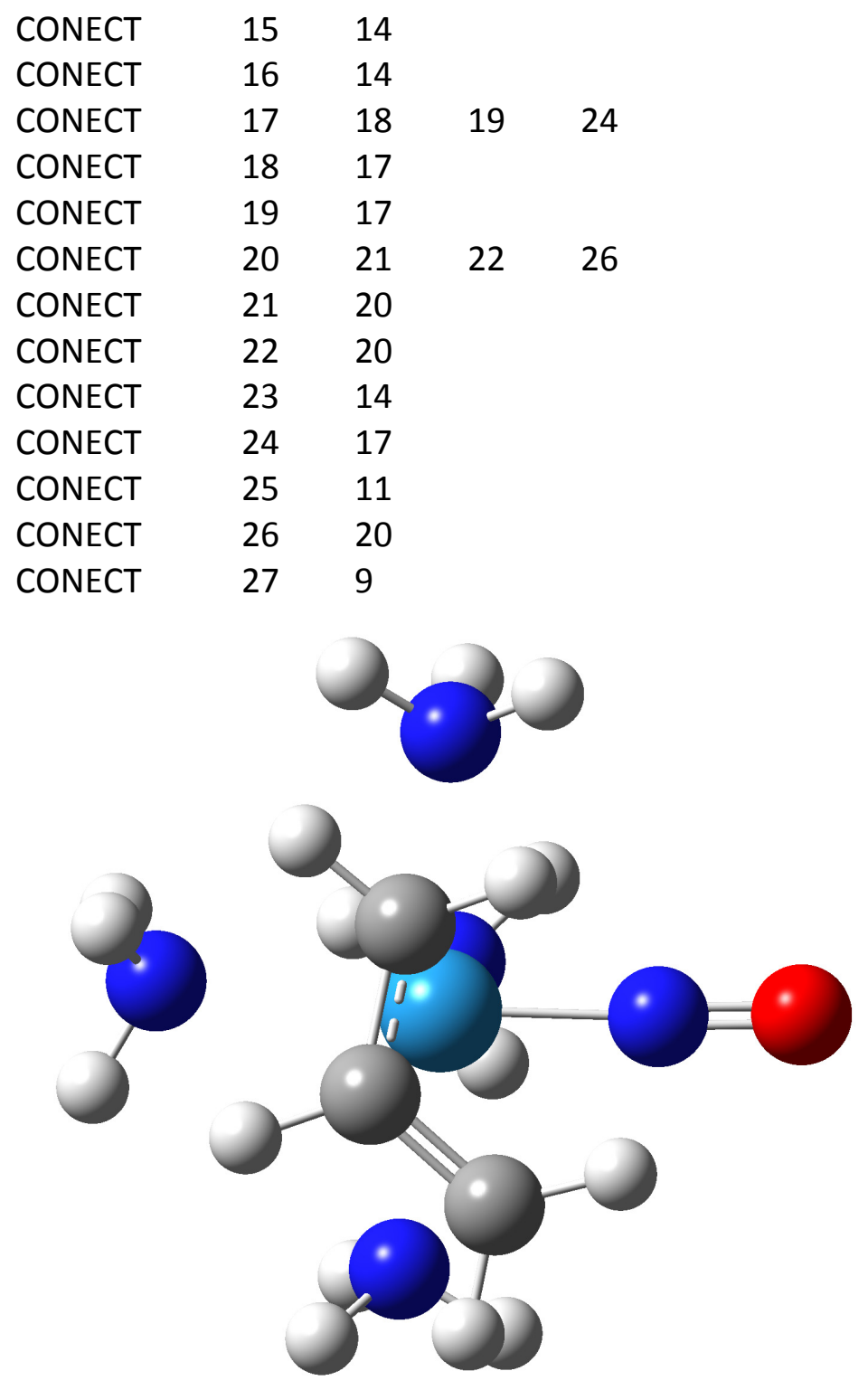




\begin{tabular}{|c|c|c|c|c|c|c|c|}
\hline TITLE & \multicolumn{7}{|c|}{$\left[\left(\mathrm{NH}_{3}\right)_{4} \mathrm{~W}(\mathrm{NO})\left(\mathrm{C}_{3} \mathrm{H}_{5}\right)\right]^{2+}$ Terminus 2} \\
\hline REMARK & 1 & \multicolumn{6}{|c|}{ File created by GaussView 5.0.8 } \\
\hline HETATM & 1 & W & 0 & -0.105 & -0.041 & 0.022 & W \\
\hline HETATM & 2 & $\mathrm{~N}$ & 0 & 0.128 & 1.737 & -0.059 & $\mathrm{~N}$ \\
\hline HETATM & 3 & $\mathrm{O}$ & 0 & 0.251 & 2.905 & -0.153 & 0 \\
\hline HETATM & 4 & C & 0 & 2.099 & -0.827 & -0.33 & C \\
\hline HETATM & 5 & $\mathrm{H}$ & 0 & 2.228 & -1.851 & 0.017 & $\mathrm{H}$ \\
\hline HETATM & 6 & C & 0 & 1.434 & -0.549 & -1.564 & C \\
\hline HETATM & 7 & $\mathrm{H}$ & 0 & 1.223 & -1.398 & -2.214 & $\mathrm{H}$ \\
\hline HETATM & 8 & $\mathrm{~N}$ & 0 & -2.282 & 0.306 & 0.693 & $\mathrm{~N}$ \\
\hline HETATM & 9 & $\mathrm{H}$ & 0 & -2.933 & -0.485 & 0.679 & $\mathrm{H}$ \\
\hline HETATM & 10 & $\mathrm{H}$ & 0 & -2.327 & 0.673 & 1.649 & $\mathrm{H}$ \\
\hline HETATM & 11 & $\mathrm{~N}$ & 0 & 0.138 & -0.274 & 2.293 & $N$ \\
\hline HETATM & 12 & $\mathrm{H}$ & 0 & -0.71 & -0.547 & 2.799 & $\mathrm{H}$ \\
\hline HETATM & 13 & $\mathrm{H}$ & 0 & 0.842 & -0.96 & 2.578 & $\mathrm{H}$ \\
\hline HETATM & 14 & $\mathrm{~N}$ & 0 & -0.594 & -2.347 & 0.027 & $\mathrm{~N}$ \\
\hline HETATM & 15 & $\mathrm{H}$ & 0 & -1.573 & -2.604 & -0.128 & $\mathrm{H}$ \\
\hline HETATM & 16 & $\mathrm{H}$ & 0 & -0.082 & -2.835 & -0.715 & $\mathrm{H}$ \\
\hline HETATM & 17 & $\mathrm{~N}$ & 0 & -1.215 & 0.029 & -1.978 & $\mathrm{~N}$ \\
\hline HETATM & 18 & $\mathrm{H}$ & 0 & -2.213 & 0.253 & -1.948 & $\mathrm{H}$ \\
\hline HETATM & 19 & $\mathrm{H}$ & 0 & -0.797 & 0.766 & -2.554 & $\mathrm{H}$ \\
\hline HETATM & 20 & $\mathrm{H}$ & 0 & 0.439 & 0.606 & 2.722 & $\mathrm{H}$ \\
\hline HETATM & 21 & $\mathrm{H}$ & 0 & -0.333 & -2.847 & 0.882 & $\mathrm{H}$ \\
\hline HETATM & 22 & $\mathrm{H}$ & 0 & -2.732 & 1.043 & 0.141 & $\mathrm{H}$ \\
\hline HETATM & 23 & $\mathrm{H}$ & 0 & -1.144 & -0.819 & -2.545 & $\mathrm{H}$ \\
\hline HETATM & 24 & $\mathrm{H}$ & 0 & 1.73 & 0.348 & -2.108 & $\mathrm{H}$ \\
\hline HETATM & 25 & C & 0 & 2.398 & 0.221 & 0.527 & C \\
\hline HETATM & 26 & $\mathrm{H}$ & 0 & 2.773 & 0.028 & 1.529 & $\mathrm{H}$ \\
\hline HETATM & 27 & $\mathrm{H}$ & 0 & 2.548 & 1.23 & 0.154 & $\mathrm{H}$ \\
\hline \multicolumn{8}{|l|}{ END } \\
\hline CONECT & 1 & 2 & & & & & \\
\hline CONECT & 2 & 1 & 3 & & & & \\
\hline CONECT & 3 & 2 & & & & & \\
\hline CONECT & 4 & 5 & 6 & 25 & & & \\
\hline CONECT & 5 & 4 & & & & & \\
\hline CONECT & 6 & 4 & 7 & 24 & & & \\
\hline CONECT & 7 & 6 & & & & & \\
\hline CONECT & 8 & 9 & 10 & 22 & & & \\
\hline CONECT & 9 & 8 & & & & & \\
\hline CONECT & 10 & 8 & & & & & \\
\hline CONECT & 11 & 12 & 13 & 20 & & & \\
\hline CONECT & 12 & 11 & & & & & \\
\hline CONECT & 13 & 11 & & & & & \\
\hline CONECT & 14 & 15 & 16 & 21 & & & \\
\hline
\end{tabular}




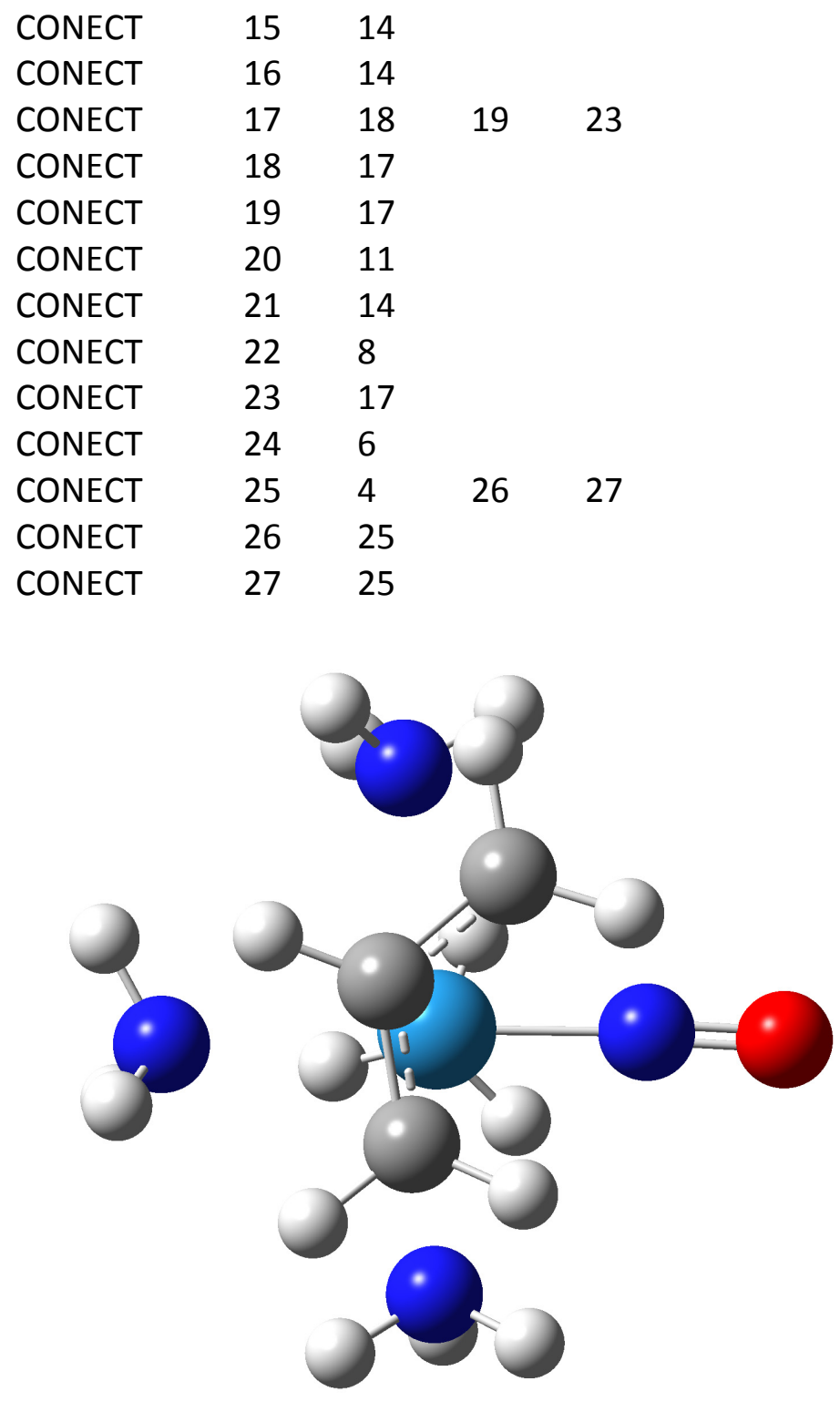




\begin{tabular}{|c|c|c|c|c|c|c|c|}
\hline TITLE & {$[(\mathbf{N}$} & W(I & $\left(C_{3}\right)$ & ${ }^{2+}$ Trans & ition Sta & ate & \\
\hline REMARK & 1 & File & ate & y Gauss & View 5.0 & 0.8 & \\
\hline HETATM & 1 & W & 0 & -0.096 & -0.061 & 0 & W \\
\hline HETATM & 2 & $\mathrm{~N}$ & 0 & -0.215 & 1.724 & 0.001 & $\mathrm{~N}$ \\
\hline HETATM & 3 & 0 & 0 & -0.297 & 2.899 & 0.001 & 0 \\
\hline HETATM & 4 & $C$ & 0 & 1.979 & 0.203 & -1.218 & C \\
\hline HETATM & 5 & C & 0 & 2.286 & -0.425 & -0.005 & C \\
\hline HETATM & 6 & C & 0 & 1.984 & 0.204 & 1.21 & C \\
\hline HETATM & 7 & $\mathrm{~N}$ & 0 & -0.564 & -0.14 & 2.248 & $\mathrm{~N}$ \\
\hline HETATM & 8 & $\mathrm{~N}$ & 0 & -2.392 & -0.282 & 0.003 & $\mathrm{~N}$ \\
\hline HETATM & 9 & $\mathrm{~N}$ & 0 & -0.574 & -0.139 & -2.245 & $\mathrm{~N}$ \\
\hline HETATM & 10 & $\mathrm{~N}$ & 0 & -0.054 & -2.419 & 0 & $\mathrm{~N}$ \\
\hline HETATM & 11 & $\mathrm{H}$ & 0 & 2.071 & 1.284 & 1.3 & $\mathrm{H}$ \\
\hline HETATM & 12 & $\mathrm{H}$ & 0 & 2.134 & -0.354 & 2.131 & $\mathrm{H}$ \\
\hline HETATM & 13 & $\mathrm{H}$ & 0 & 2.546 & -1.483 & -0.005 & $\mathrm{H}$ \\
\hline HETATM & 14 & $\mathrm{H}$ & 0 & 2.125 & -0.356 & -2.139 & $\mathrm{H}$ \\
\hline HETATM & 15 & $\mathrm{H}$ & 0 & 2.065 & 1.284 & -1.309 & $\mathrm{H}$ \\
\hline HETATM & 16 & $\mathrm{H}$ & 0 & -1.564 & -0.033 & -2.482 & $\mathrm{H}$ \\
\hline HETATM & 17 & $\mathrm{H}$ & 0 & -0.118 & 0.651 & -2.71 & $\mathrm{H}$ \\
\hline HETATM & 18 & $\mathrm{H}$ & 0 & -0.258 & -0.967 & -2.757 & $\mathrm{H}$ \\
\hline HETATM & 19 & $\mathrm{H}$ & 0 & -2.791 & -0.76 & 0.816 & $\mathrm{H}$ \\
\hline HETATM & 20 & $\mathrm{H}$ & 0 & -2.79 & -0.786 & -0.795 & $\mathrm{H}$ \\
\hline HETATM & 21 & $\mathrm{H}$ & 0 & -2.841 & 0.64 & -0.012 & $\mathrm{H}$ \\
\hline HETATM & 22 & $\mathrm{H}$ & 0 & 0.439 & -2.814 & 0.807 & $\mathrm{H}$ \\
\hline HETATM & 23 & $\mathrm{H}$ & 0 & 0.437 & -2.815 & -0.807 & $\mathrm{H}$ \\
\hline HETATM & 24 & $\mathrm{H}$ & 0 & -0.964 & -2.889 & 0.002 & $\mathrm{H}$ \\
\hline HETATM & 25 & $\mathrm{H}$ & 0 & -0.254 & -0.972 & 2.757 & $\mathrm{H}$ \\
\hline HETATM & 26 & $\mathrm{H}$ & 0 & -1.553 & -0.025 & 2.489 & $\mathrm{H}$ \\
\hline HETATM & 27 & $\mathrm{H}$ & 0 & -0.1 & 0.645 & 2.713 & $\mathrm{H}$ \\
\hline END & & & & & & & \\
\hline CONECT & 1 & 2 & & & & & \\
\hline CONECT & 2 & 1 & 3 & & & & \\
\hline CONECT & 3 & 2 & & & & & \\
\hline CONECT & 4 & 5 & 14 & 15 & & & \\
\hline CONECT & 5 & 4 & 6 & 13 & & & \\
\hline CONECT & 6 & 5 & 11 & 12 & & & \\
\hline CONECT & 7 & 25 & 26 & 27 & & & \\
\hline CONECT & 8 & 19 & 20 & 21 & & & \\
\hline CONECT & 9 & 16 & 17 & 18 & & & \\
\hline CONECT & 10 & 22 & 23 & 24 & & & \\
\hline CONECT & 11 & 6 & & & & & \\
\hline CONECT & 12 & 6 & & & & & \\
\hline CONECT & 13 & 5 & & & & & \\
\hline CONECT & 14 & 4 & & & & & \\
\hline
\end{tabular}




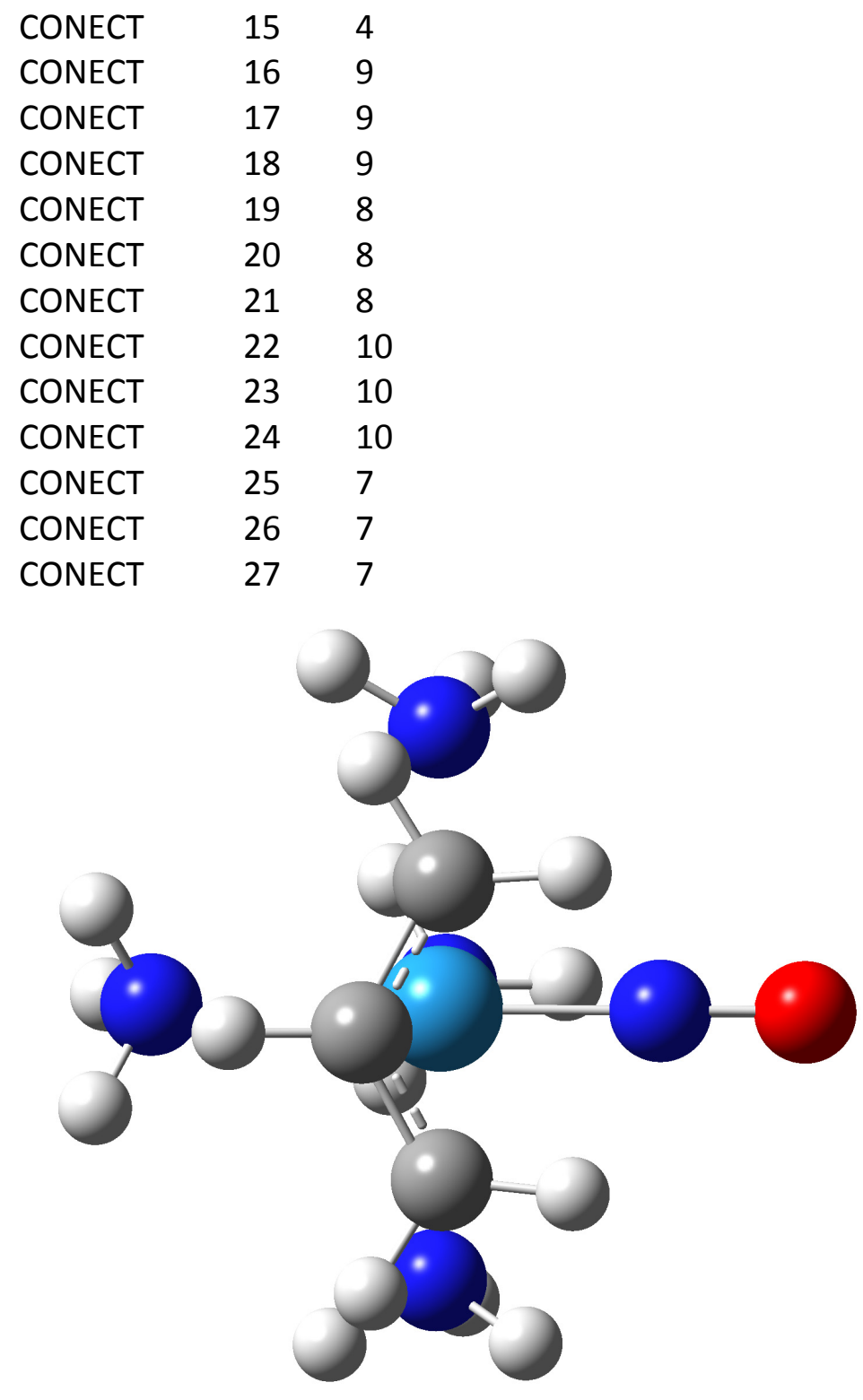




\begin{tabular}{|c|c|c|c|c|c|c|c|}
\hline TITLE & \multicolumn{7}{|c|}{$\left[\left(\mathrm{NH}_{3}\right)_{4} \mathrm{~W}(\mathrm{NO})\left(\mathrm{C}_{6} \mathrm{H}_{9}\right)\right]^{2+}$ Terminus 1} \\
\hline REMARK & 1 & \multicolumn{6}{|c|}{ File created by GaussView 5.0.8 } \\
\hline HETATM & 1 & W & 0 & 0.537 & 0.002 & -0.001 & W \\
\hline HETATM & 2 & $\mathrm{~N}$ & 0 & -0.327 & 1.383 & -0.739 & $\mathrm{~N}$ \\
\hline HETATM & 3 & $\mathrm{O}$ & 0 & -0.835 & 2.333 & -1.235 & $\mathrm{O}$ \\
\hline HETATM & 4 & C & 0 & -1.103 & -1.521 & 0.114 & C \\
\hline HETATM & 5 & $\mathrm{H}$ & 0 & -0.693 & -2.534 & 0.105 & $\mathrm{H}$ \\
\hline HETATM & 6 & C & 0 & -1.066 & -0.87 & 1.408 & C \\
\hline HETATM & 7 & $\mathrm{H}$ & 0 & -0.56 & -1.296 & 2.274 & $\mathrm{H}$ \\
\hline HETATM & 8 & C & 0 & -1.768 & 0.323 & 1.55 & C \\
\hline HETATM & 9 & $\mathrm{H}$ & 0 & -1.653 & 0.888 & 2.476 & $\mathrm{H}$ \\
\hline HETATM & 10 & $\mathrm{~N}$ & 0 & 2.552 & 0.795 & -0.827 & $N$ \\
\hline HETATM & 11 & $\mathrm{H}$ & 0 & 3.366 & 0.713 & -0.21 & $\mathrm{H}$ \\
\hline HETATM & 12 & $\mathrm{H}$ & 0 & 2.863 & 0.382 & -1.711 & $\mathrm{H}$ \\
\hline HETATM & 13 & $\mathrm{~N}$ & 0 & 0.618 & -1.05 & -2.02 & $N$ \\
\hline HETATM & 14 & $\mathrm{H}$ & 0 & 1.113 & -0.554 & -2.767 & $\mathrm{H}$ \\
\hline HETATM & 15 & $\mathrm{H}$ & 0 & 0.981 & -2.007 & -2.023 & $\mathrm{H}$ \\
\hline HETATM & 16 & $\mathrm{~N}$ & 0 & 1.879 & -1.743 & 0.873 & $N$ \\
\hline HETATM & 17 & $\mathrm{H}$ & 0 & 2.632 & -2.069 & 0.259 & $\mathrm{H}$ \\
\hline HETATM & 18 & $\mathrm{H}$ & 0 & 2.346 & -1.541 & 1.762 & $\mathrm{H}$ \\
\hline HETATM & 19 & $\mathrm{~N}$ & 0 & 1.209 & 1.214 & 1.83 & $\mathrm{~N}$ \\
\hline HETATM & 20 & $\mathrm{H}$ & 0 & 2.193 & 1.497 & 1.839 & $\mathrm{H}$ \\
\hline HETATM & 21 & $\mathrm{H}$ & 0 & 0.685 & 2.093 & 1.88 & $\mathrm{H}$ \\
\hline HETATM & 22 & $\mathrm{H}$ & 0 & -0.344 & -1.132 & -2.358 & $\mathrm{H}$ \\
\hline HETATM & 23 & $\mathrm{H}$ & 0 & 1.321 & -2.581 & 1.063 & $\mathrm{H}$ \\
\hline HETATM & 24 & $\mathrm{H}$ & 0 & 2.461 & 1.796 & -1.032 & $\mathrm{H}$ \\
\hline HETATM & 25 & $\mathrm{H}$ & 0 & 1.054 & 0.759 & 2.734 & $\mathrm{H}$ \\
\hline HETATM & 26 & $C$ & 0 & -2.411 & -1.416 & -0.673 & C \\
\hline HETATM & 27 & C & 0 & -2.891 & 0.745 & 0.665 & C \\
\hline HETATM & 28 & $\mathrm{H}$ & 0 & -2.875 & 1.831 & 0.505 & $\mathrm{H}$ \\
\hline HETATM & 29 & $\mathrm{H}$ & 0 & -3.777 & 0.583 & 1.307 & $\mathrm{H}$ \\
\hline HETATM & 30 & $C$ & 0 & -3.032 & -0.007 & -0.675 & C \\
\hline HETATM & 31 & $\mathrm{H}$ & 0 & -2.281 & -1.761 & -1.707 & $\mathrm{H}$ \\
\hline HETATM & 32 & $\mathrm{H}$ & 0 & -3.105 & -2.139 & -0.223 & $\mathrm{H}$ \\
\hline HETATM & 33 & $\mathrm{H}$ & 0 & -4.093 & -0.081 & -0.931 & $\mathrm{H}$ \\
\hline HETATM & 34 & $\mathrm{H}$ & 0 & -2.58 & 0.597 & -1.465 & $\mathrm{H}$ \\
\hline \multicolumn{8}{|l|}{ END } \\
\hline CONECT & 1 & 2 & & & & & \\
\hline CONECT & 2 & 1 & 3 & & & & \\
\hline CONECT & 3 & 2 & & & & & \\
\hline CONECT & 4 & 5 & 6 & 26 & & & \\
\hline CONECT & 5 & 4 & & & & & \\
\hline CONECT & 6 & 4 & 7 & 8 & & & \\
\hline CONECT & 7 & 6 & & & & & \\
\hline
\end{tabular}




$\begin{array}{llllll}\text { CONECT } & 8 & 6 & 9 & 27 & \\ \text { CONECT } & 9 & 8 & & & \\ \text { CONECT } & 10 & 11 & 12 & 24 & \\ \text { CONECT } & 11 & 10 & & & \\ \text { CONECT } & 12 & 10 & & & \\ \text { CONECT } & 13 & 14 & 15 & 22 & \\ \text { CONECT } & 14 & 13 & & & \\ \text { CONECT } & 15 & 13 & & & \\ \text { CONECT } & 16 & 17 & 18 & 23 & \\ \text { CONECT } & 17 & 16 & & & \\ \text { CONECT } & 18 & 16 & & & \\ \text { CONECT } & 19 & 20 & 21 & 25 & \\ \text { CONECT } & 20 & 19 & & & \\ \text { CONECT } & 21 & 19 & & & \\ \text { CONECT } & 22 & 13 & & & \\ \text { CONECT } & 23 & 16 & & & \\ \text { CONECT } & 24 & 10 & & & \\ \text { CONECT } & 25 & 19 & & & \\ \text { CONECT } & 26 & 4 & 30 & 31 & 32 \\ \text { CONECT } & 27 & 8 & 28 & 29 & 30 \\ \text { CONECT } & 28 & 27 & & & \\ \text { CONECT } & 29 & 27 & & & \\ \text { CONECT } & 30 & 26 & 27 & 33 & 34 \\ \text { CONECT } & 31 & 26 & & & \\ \text { CONECT } & 32 & 26 & & & \\ \text { CONECT } & 33 & 30 & & & \\ \text { CONECT } & 34 & 30 & & & \end{array}$
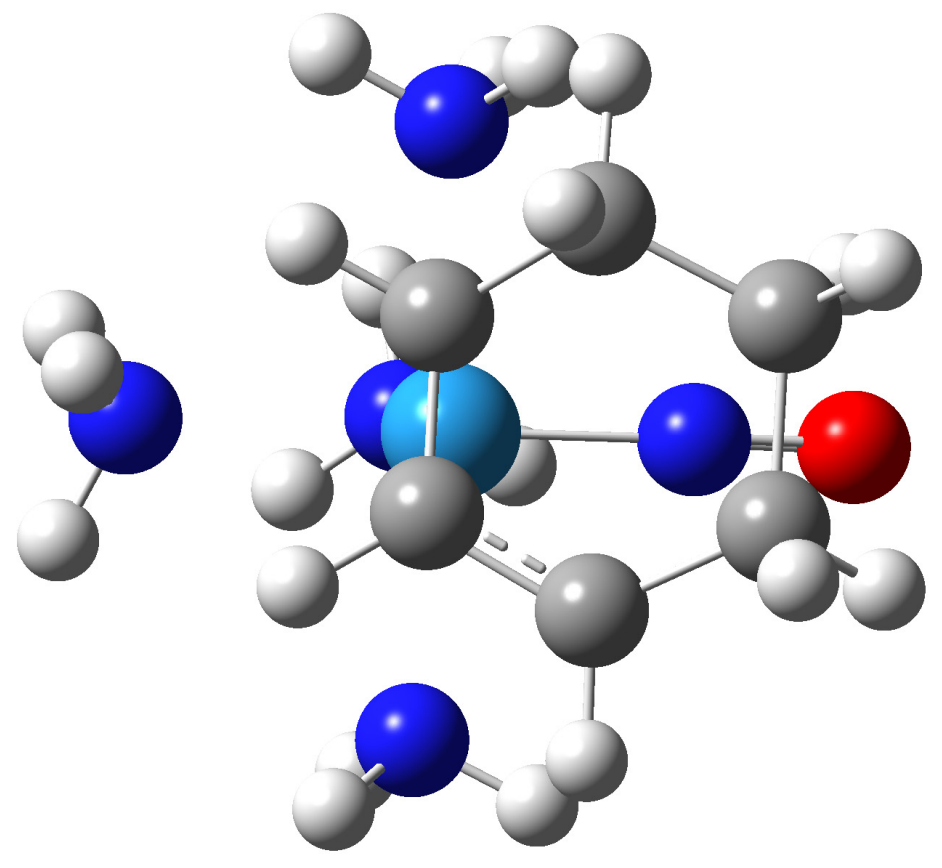


\begin{tabular}{|c|c|c|c|c|c|c|c|}
\hline TITLE & \multicolumn{7}{|c|}{$\left[\left(\mathrm{NH}_{3}\right)_{4} \mathrm{~W}(\mathrm{NO})\left(\mathrm{C}_{6} \mathrm{H}_{9}\right)\right]^{2+}$ Terminus 2} \\
\hline REMARK & 1 & \multicolumn{6}{|c|}{ File created by GaussView 5.0.8 } \\
\hline HETATM & 1 & W & 0 & -0.54 & 0.006 & 0.004 & W \\
\hline HETATM & 2 & $\mathrm{~N}$ & 0 & 0.328 & 1.407 & -0.689 & $\mathrm{~N}$ \\
\hline HETATM & 3 & 0 & 0 & 0.842 & 2.367 & -1.161 & $\mathrm{O}$ \\
\hline HETATM & 4 & $\mathrm{C}$ & 0 & 1.072 & -0.9 & 1.384 & $\mathrm{C}$ \\
\hline HETATM & 5 & $\mathrm{H}$ & 0 & 0.574 & -1.345 & 2.244 & $\mathrm{H}$ \\
\hline HETATM & 6 & C & 0 & 1.094 & -1.519 & 0.072 & C \\
\hline HETATM & 7 & $\mathrm{H}$ & 0 & 0.677 & -2.53 & 0.046 & $\mathrm{H}$ \\
\hline HETATM & 8 & C & 0 & 2.398 & -1.405 & -0.72 & C \\
\hline HETATM & 9 & $\mathrm{~N}$ & 0 & -2.523 & 0.841 & -0.849 & $\mathrm{~N}$ \\
\hline HETATM & 10 & $\mathrm{H}$ & 0 & -3.31 & 0.197 & -0.971 & $\mathrm{H}$ \\
\hline HETATM & 11 & $\mathrm{H}$ & 0 & -2.893 & 1.61 & -0.284 & $\mathrm{H}$ \\
\hline HETATM & 12 & $\mathrm{~N}$ & 0 & -1.215 & 1.133 & 1.888 & $N$ \\
\hline HETATM & 13 & $\mathrm{H}$ & 0 & -0.799 & 2.069 & 1.916 & $\mathrm{H}$ \\
\hline HETATM & 14 & $\mathrm{H}$ & 0 & -2.223 & 1.286 & 1.984 & $\mathrm{H}$ \\
\hline HETATM & 15 & $\mathrm{~N}$ & 0 & -1.885 & -1.765 & 0.821 & $N$ \\
\hline HETATM & 16 & $\mathrm{H}$ & 0 & -2.592 & -1.505 & 1.515 & $\mathrm{H}$ \\
\hline HETATM & 17 & $\mathrm{H}$ & 0 & -2.408 & -2.273 & 0.102 & $\mathrm{H}$ \\
\hline HETATM & 18 & $\mathrm{~N}$ & 0 & -0.632 & -1.004 & -2.037 & $N$ \\
\hline HETATM & 19 & $\mathrm{H}$ & 0 & -1.34 & -0.676 & -2.699 & $\mathrm{H}$ \\
\hline HETATM & 20 & $\mathrm{H}$ & 0 & 0.264 & -0.833 & -2.5 & $\mathrm{H}$ \\
\hline HETATM & 21 & $\mathrm{H}$ & 0 & -0.934 & 0.687 & 2.765 & $\mathrm{H}$ \\
\hline HETATM & 22 & $\mathrm{H}$ & 0 & -1.324 & -2.487 & 1.283 & $\mathrm{H}$ \\
\hline HETATM & 23 & $\mathrm{H}$ & 0 & -2.37 & 1.271 & -1.766 & $\mathrm{H}$ \\
\hline HETATM & 24 & $\mathrm{H}$ & 0 & -0.723 & -2.023 & -2.015 & $\mathrm{H}$ \\
\hline HETATM & 25 & C & 0 & 1.79 & 0.279 & 1.554 & $C$ \\
\hline HETATM & 26 & $C$ & 0 & 3.027 & 0 & -0.692 & C \\
\hline HETATM & 27 & $\mathrm{H}$ & 0 & 2.572 & 0.627 & -1.462 & $\mathrm{H}$ \\
\hline HETATM & 28 & $\mathrm{H}$ & 0 & 4.085 & -0.074 & -0.961 & $\mathrm{H}$ \\
\hline HETATM & 29 & $C$ & 0 & 2.904 & 0.718 & 0.667 & C \\
\hline HETATM & 30 & $\mathrm{H}$ & 0 & 3.796 & 0.536 & 1.295 & $\mathrm{H}$ \\
\hline HETATM & 31 & $\mathrm{H}$ & 0 & 2.892 & 1.808 & 0.537 & $\mathrm{H}$ \\
\hline HETATM & 32 & $\mathrm{H}$ & 0 & 2.257 & -1.725 & -1.76 & $\mathrm{H}$ \\
\hline HETATM & 33 & $\mathrm{H}$ & 0 & 3.092 & -2.141 & -0.289 & $\mathrm{H}$ \\
\hline HETATM & 34 & $\mathrm{H}$ & 0 & 1.69 & 0.82 & 2.496 & $\mathrm{H}$ \\
\hline \multicolumn{8}{|l|}{ END } \\
\hline CONECT & 1 & 2 & & & & & \\
\hline CONECT & 2 & 1 & 3 & & & & \\
\hline CONECT & 3 & 2 & & & & & \\
\hline CONECT & 4 & 5 & 6 & 25 & & & \\
\hline CONECT & 5 & 4 & & & & & \\
\hline CONECT & 6 & 4 & 7 & 8 & & & \\
\hline CONECT & 7 & 6 & & & & & \\
\hline
\end{tabular}




$\begin{array}{llllll}\text { CONECT } & 8 & 6 & 26 & 32 & 33 \\ \text { CONECT } & 9 & 10 & 11 & 23 & \\ \text { CONECT } & 10 & 9 & & & \\ \text { CONECT } & 11 & 9 & & & \\ \text { CONECT } & 12 & 13 & 14 & 21 & \\ \text { CONECT } & 13 & 12 & & & \\ \text { CONECT } & 14 & 12 & & & \\ \text { CONECT } & 15 & 16 & 17 & 22 & \\ \text { CONECT } & 16 & 15 & & & \\ \text { CONECT } & 17 & 15 & & & \\ \text { CONECT } & 18 & 19 & 20 & 24 & \\ \text { CONECT } & 19 & 18 & & & \\ \text { CONECT } & 20 & 18 & & & \\ \text { CONECT } & 21 & 12 & & & \\ \text { CONECT } & 22 & 15 & & & \\ \text { CONECT } & 23 & 9 & & & \\ \text { CONECT } & 24 & 18 & & & \\ \text { CONECT } & 25 & 4 & 29 & 34 & \\ \text { CONECT } & 26 & 8 & 27 & 28 & 29 \\ \text { CONECT } & 27 & 26 & & & \\ \text { CONECT } & 28 & 26 & & & \\ \text { CONECT } & 29 & 26 & 25 & 30 & 31 \\ \text { CONECT } & 30 & 29 & & & \\ \text { CONECT } & 31 & 29 & & & \\ \text { CONECT } & 32 & 8 & & & \\ \text { CONECT } & 33 & 8 & & & \\ \text { CONECT } & 34 & 25 & & & \end{array}$
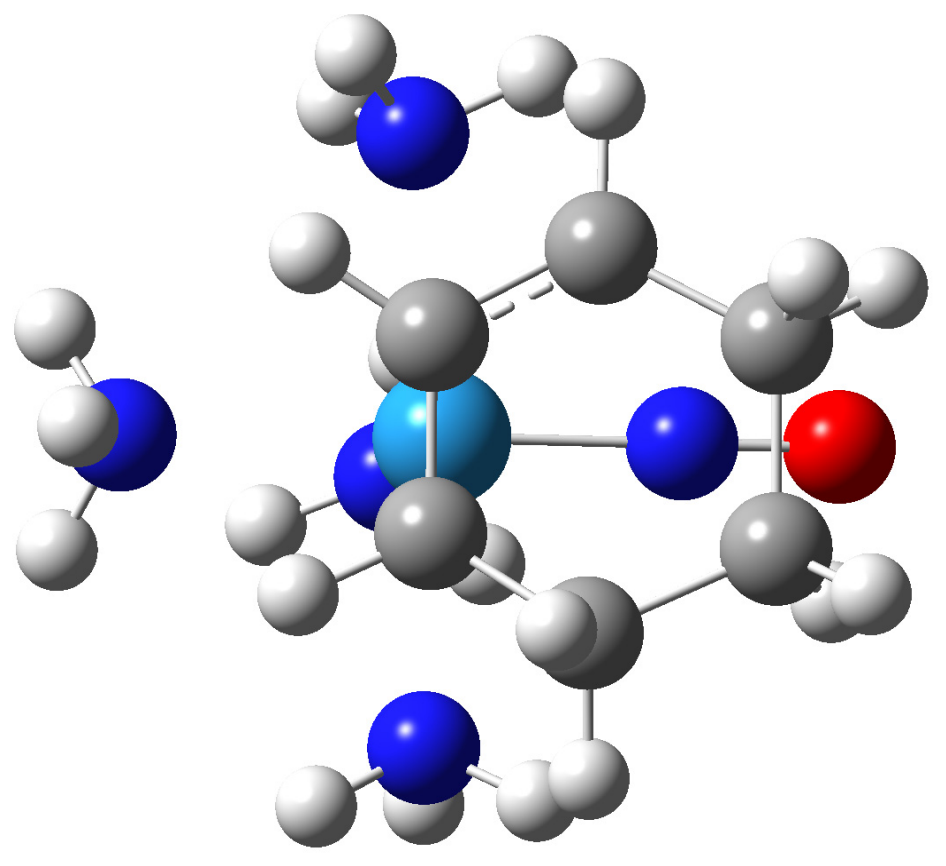


\begin{tabular}{|c|c|c|c|c|c|c|c|}
\hline TITLE & \multicolumn{7}{|c|}{$\left[\left(\mathrm{NH}_{3}\right)_{4} \mathrm{~W}(\mathrm{NO})\left(\mathrm{C}_{6} \mathrm{H}_{9}\right)\right]^{2+}$ Transition State } \\
\hline REMARK & 1 & \multicolumn{6}{|c|}{ File created by GaussView 5.0.8 } \\
\hline HETATM & 1 & W & 0 & -0.51 & -0.028 & -0.001 & W \\
\hline HETATM & 2 & $\mathrm{~N}$ & 0 & 0.272 & 1.576 & -0.03 & $\mathrm{~N}$ \\
\hline HETATM & 3 & $\mathrm{O}$ & 0 & 0.614 & 2.711 & -0.048 & $\mathrm{O}$ \\
\hline HETATM & 4 & $\mathrm{C}$ & 0 & 1.417 & -1.016 & 1.221 & C \\
\hline HETATM & 5 & C & 0 & 1.134 & -1.71 & 0.036 & C \\
\hline HETATM & 6 & C & 0 & 1.42 & -1.065 & -1.177 & C \\
\hline HETATM & 7 & $\mathrm{~N}$ & 0 & -2.516 & 1.119 & -0.019 & $N$ \\
\hline HETATM & 8 & $\mathrm{~N}$ & 0 & -0.916 & 0.171 & 2.248 & $N$ \\
\hline HETATM & 9 & $\mathrm{~N}$ & 0 & -1.915 & -1.936 & 0.037 & $\mathrm{~N}$ \\
\hline HETATM & 10 & $\mathrm{~N}$ & 0 & -0.906 & 0.082 & -2.259 & $N$ \\
\hline HETATM & 11 & $C$ & 0 & 2.63 & -0.111 & 1.315 & C \\
\hline HETATM & 12 & $\mathrm{C}$ & 0 & 3.079 & 0.552 & -0.008 & C \\
\hline HETATM & 13 & $C$ & 0 & 2.632 & -0.164 & -1.305 & C \\
\hline HETATM & 14 & $\mathrm{H}$ & 0 & 1.121 & -1.499 & 2.153 & $\mathrm{H}$ \\
\hline HETATM & 15 & $\mathrm{H}$ & 0 & 0.609 & -2.663 & 0.055 & $\mathrm{H}$ \\
\hline HETATM & 16 & $\mathrm{H}$ & 0 & 1.126 & -1.586 & -2.089 & $\mathrm{H}$ \\
\hline HETATM & 17 & $\mathrm{H}$ & 0 & -3.151 & 0.936 & 0.763 & $\mathrm{H}$ \\
\hline HETATM & 18 & $\mathrm{H}$ & 0 & -3.102 & 0.989 & -0.849 & $\mathrm{H}$ \\
\hline HETATM & 19 & $\mathrm{H}$ & 0 & -2.33 & 2.128 & 0.017 & $\mathrm{H}$ \\
\hline HETATM & 20 & $\mathrm{H}$ & 0 & -2.921 & -1.748 & 0.029 & $\mathrm{H}$ \\
\hline HETATM & 21 & $\mathrm{H}$ & 0 & -1.765 & -2.569 & -0.755 & $\mathrm{H}$ \\
\hline HETATM & 22 & $\mathrm{H}$ & 0 & -1.77 & -2.53 & 0.86 & $\mathrm{H}$ \\
\hline HETATM & 23 & $\mathrm{H}$ & 0 & -1.702 & 0.662 & -2.539 & $\mathrm{H}$ \\
\hline HETATM & 24 & $\mathrm{H}$ & 0 & -0.099 & 0.523 & -2.706 & $\mathrm{H}$ \\
\hline HETATM & 25 & $\mathrm{H}$ & 0 & -1.033 & -0.807 & -2.75 & $\mathrm{H}$ \\
\hline HETATM & 26 & $\mathrm{H}$ & 0 & -1.713 & 0.762 & 2.5 & $\mathrm{H}$ \\
\hline HETATM & 27 & $\mathrm{H}$ & 0 & -1.047 & -0.698 & 2.774 & $\mathrm{H}$ \\
\hline HETATM & 28 & $\mathrm{H}$ & 0 & 2.725 & 1.582 & -0.03 & $\mathrm{H}$ \\
\hline HETATM & 29 & $\mathrm{H}$ & 0 & 4.17 & 0.622 & -0.009 & $\mathrm{H}$ \\
\hline HETATM & 30 & $\mathrm{H}$ & 0 & 3.423 & -0.767 & 1.706 & $\mathrm{H}$ \\
\hline HETATM & 31 & $\mathrm{H}$ & 0 & 2.493 & 0.655 & 2.088 & $\mathrm{H}$ \\
\hline HETATM & 32 & $\mathrm{H}$ & 0 & 2.496 & 0.57 & -2.108 & $\mathrm{H}$ \\
\hline HETATM & 33 & $\mathrm{H}$ & 0 & 3.426 & -0.835 & -1.668 & $\mathrm{H}$ \\
\hline HETATM & 34 & $\mathrm{H}$ & 0 & -0.111 & 0.628 & 2.683 & $\mathrm{H}$ \\
\hline \multicolumn{8}{|l|}{ END } \\
\hline CONECT & 1 & 2 & & & & & \\
\hline CONECT & 2 & 1 & 3 & & & & \\
\hline CONECT & 3 & 2 & & & & & \\
\hline CONECT & 4 & 5 & 11 & 14 & & & \\
\hline CONECT & 5 & 4 & 6 & 15 & & & \\
\hline CONECT & 6 & 5 & 13 & 16 & & & \\
\hline CONECT & 7 & 17 & 18 & 19 & & & \\
\hline
\end{tabular}




$\begin{array}{llllll}\text { CONECT } & 8 & 26 & 27 & 34 & \\ \text { CONECT } & 9 & 20 & 21 & 22 & \\ \text { CONECT } & 10 & 23 & 24 & 25 & \\ \text { CONECT } & 11 & 4 & 12 & 30 & 31 \\ \text { CONECT } & 12 & 11 & 13 & 28 & 29 \\ \text { CONECT } & 13 & 6 & 12 & 32 & 33 \\ \text { CONECT } & 14 & 4 & & & \\ \text { CONECT } & 15 & 5 & & & \\ \text { CONECT } & 16 & 6 & & & \\ \text { CONECT } & 17 & 7 & & & \\ \text { CONECT } & 18 & 7 & & & \\ \text { CONECT } & 19 & 7 & & & \\ \text { CONECT } & 20 & 9 & & & \\ \text { CONECT } & 21 & 9 & & & \\ \text { CONECT } & 22 & 9 & & \\ \text { CONECT } & 23 & 10 & & \\ \text { CONECT } & 24 & 10 & & \\ \text { CONECT } & 25 & 10 & & \\ \text { CONECT } & 26 & 8 & & \\ \text { CONECT } & 27 & 8 & & \\ \text { CONECT } & 28 & 12 & & \\ \text { CONECT } & 29 & 12 & & \\ \text { CONECT } & 30 & 11 & & \\ \text { CONECT } & 31 & 11 & & \\ \text { CONECT } & 32 & 13 & & \\ \text { CONECT } & 33 & 13 & & \\ \text { CONECT } & 34 & 8 & & & \end{array}$


TITLE

REMARK 1 File created

HETATM

HETATM

HETATM

HETATM

HETATM

HETATM

HETATM

HETATM

HETATM

HETATM 10

HETATM 11

HETATM 12

HETATM 13

HETATM 14

HETATM 15

HETATM 16

HETATM 17

HETATM 18

HETATM 19

HETATM $20 \quad \mathrm{~N}$

HETATM 21

HETATM 22

HETATM 23

HETATM $24 \quad \mathrm{H}$

HETATM 25

HETATM $26 \quad$

HETATM 27

HETATM $28 \quad \mathrm{H}$

HETATM 29

HETATM 30

HETATM 31

HETATM 32

HETATM 33

HETATM 34

HETATM 35

HETATM 36

HETATM 37

HETATM 38

HETATM 39

$\begin{array}{llll}\text { HETATM } & 40 & \mathrm{H} & 0\end{array}$

HETATM 41 C 0

$\begin{array}{llll}\text { HETATM } & 42 & \mathrm{H} & 0\end{array}$ $\begin{array}{lll}-0.37 & -0.414 & -0.51 \quad W\end{array}$

$\begin{array}{llll}-2.621 & 0.387 & -0.038 P\end{array}$

$\begin{array}{llll}-3.743 & -0.888 & 0.706 \quad C\end{array}$

$\begin{array}{lllll}-4.785 & -0.549 & 0.737 \quad \mathrm{H}\end{array}$

$\begin{array}{llll}-3.671 & -1.8130 .127 \quad H\end{array}$

$-3.413-1.1071 .726 \quad \mathrm{H}$

$-3.7141 .013-1.421 \mathrm{C}$

$-4.7091 .307-1.063 \mathrm{H}$

$-3.2481 .878-1.906 \mathrm{H}$

$\begin{array}{lll}-3.827 & 0.225 & -2.173 \mathrm{H}\end{array}$

$\begin{array}{llll}-2.794 & 1.791 & 1.17 \quad \mathrm{C}\end{array}$

$\begin{array}{llll}-3.844 & 2.052 & 1.343 \quad \mathrm{H}\end{array}$

$\begin{array}{llll}-2.333 & 1.502 & 2.12 \quad \mathrm{H}\end{array}$

$\begin{array}{llll}-2.265 & 2.673 & 0.796 \quad H\end{array}$

$\begin{array}{llll}-1.022 & -2.082 & -0.589 \mathrm{~N}\end{array}$

$-1.514-3.187-0.6610$

$\begin{array}{llll}1.442 & 0.15 & 1.865 \quad \mathrm{~N}\end{array}$

$\begin{array}{lllll}0.26 & -0.456 & 1.526 \quad \mathrm{~N}\end{array}$

$\begin{array}{llll}1.61 & 1.949 & 0.086 & N\end{array}$

$\begin{array}{lll}0.447 & 1.689 & -0.574 \mathrm{~N}\end{array}$

$\begin{array}{llll}1.676 & -0.03 & 3.19 \quad \mathrm{C}\end{array}$

$\begin{array}{lllll}2.559 & 0.393 & 3.646 \quad \mathrm{H}\end{array}$

$-0.221-1.0212 .645 \quad C$

$\begin{array}{lllll}-1.15 & -1.569 & 2.608 \quad \mathrm{H}\end{array}$

$\begin{array}{lll}1.942 & 3.245 & -0.069 \mathrm{C}\end{array}$

$\begin{array}{lllll}2.838 & 3.638 & 0.391 & \mathrm{H}\end{array}$

$0.0612 .844 \quad-1.143 \mathrm{C}$

$\begin{array}{lll}-0.85 & 2.88 & -1.724 \mathrm{H}\end{array}$

$\begin{array}{lllll}0.642 & -0.779 & 3.724 \quad C\end{array}$

$0.523 \quad-1.102 \quad 4.748 \quad \mathrm{H}$

$\begin{array}{lllll}2.367 & 0.802 & 0.812 & B\end{array}$

$\begin{array}{llll}3.352 & 1.24 & 1.342 \quad \mathrm{H}\end{array}$

$\begin{array}{llll}0.976 & 3.863 & -0.855 \mathrm{C}\end{array}$

$0.9414 .895-1.168 \mathrm{H}$

$1.749-0.909-0.916 \mathrm{~N}$

$2.741-0.263-0.239 \mathrm{~N}$

$\begin{array}{lllll}2.349 & -1.81 & -1.71 & C\end{array}$

$1.749-2.455-2.337 \mathrm{H}$

$3.946-0.752-0.612 \mathrm{C}$

$\begin{array}{lllll}4.854 & -0.356 & -0.18 \quad \mathrm{H}\end{array}$

$3.741-1.748-1.555 \mathrm{C}$

$4.49-2.345-2.055 \mathrm{H}$ 
END

\begin{tabular}{|c|c|c|c|c|c|}
\hline CONECT & 1 & 2 & 15 & & \\
\hline CONECT & 2 & 1 & 3 & 7 & 11 \\
\hline CONECT & 3 & 2 & 4 & 5 & 6 \\
\hline CONECT & 4 & 3 & & & \\
\hline CONECT & 5 & 3 & & & \\
\hline CONECT & 6 & 3 & & & \\
\hline CONECT & 7 & 2 & 8 & 9 & 10 \\
\hline CONECT & 8 & 7 & & & \\
\hline CONECT & 9 & 7 & & & \\
\hline CONECT & 10 & 7 & & & \\
\hline CONECT & 11 & 2 & 12 & 13 & 14 \\
\hline CONECT & 12 & 11 & & & \\
\hline CONECT & 13 & 11 & & & \\
\hline CONECT & 14 & 11 & & & \\
\hline CONECT & 15 & 1 & 16 & & \\
\hline CONECT & 16 & 15 & & & \\
\hline CONECT & 17 & 18 & 21 & 31 & \\
\hline CONECT & 18 & 17 & 23 & & \\
\hline CONECT & 19 & 20 & 25 & 31 & \\
\hline CONECT & 20 & 19 & 27 & & \\
\hline CONECT & 21 & 17 & 22 & 29 & \\
\hline CONECT & 22 & 21 & & & \\
\hline CONECT & 23 & 18 & 24 & 29 & \\
\hline CONECT & 24 & 23 & & & \\
\hline CONECT & 25 & 19 & 26 & 33 & \\
\hline CONECT & 26 & 25 & & & \\
\hline CONECT & 27 & 20 & 28 & 33 & \\
\hline CONECT & 28 & 27 & & & \\
\hline CONECT & 29 & 23 & 21 & 30 & \\
\hline CONECT & 30 & 29 & & & \\
\hline CONECT & 31 & 19 & 17 & 32 & 36 \\
\hline CONECT & 32 & 31 & & & \\
\hline CONECT & 33 & 25 & 27 & 34 & \\
\hline CONECT & 34 & 33 & & & \\
\hline CONECT & 35 & 36 & 37 & & \\
\hline CONECT & 36 & 35 & 31 & 39 & \\
\hline CONECT & 37 & 35 & 38 & 41 & \\
\hline CONECT & 38 & 37 & & & \\
\hline CONECT & 39 & 36 & 40 & 41 & \\
\hline CONECT & 40 & 39 & & & \\
\hline CONECT & 41 & 37 & 39 & 42 & \\
\hline CONECT & 42 & 41 & & & \\
\hline
\end{tabular}

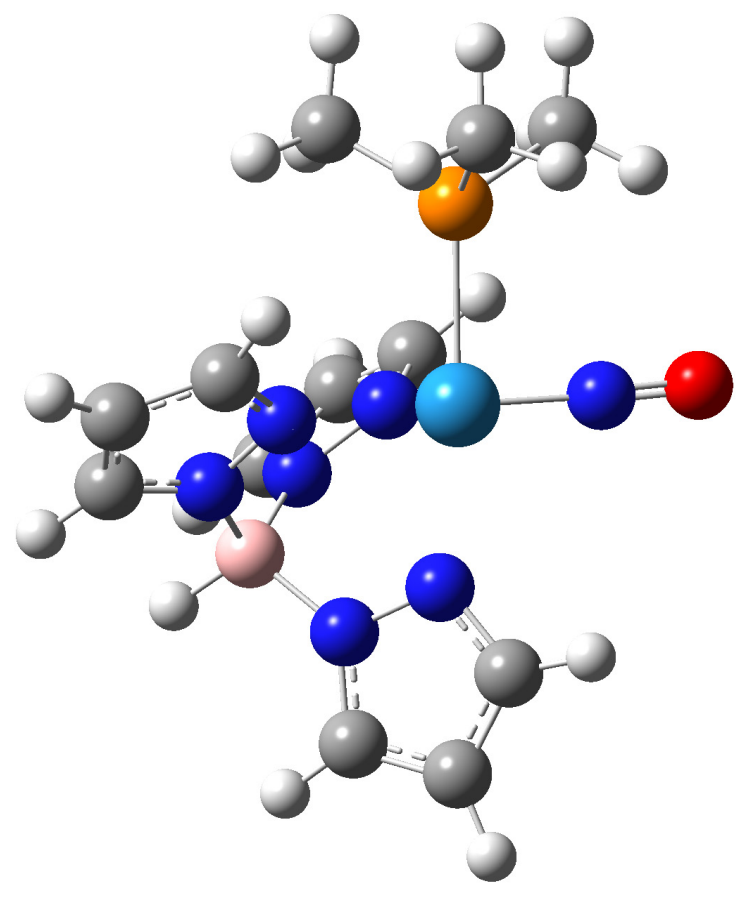


HOMO of $\left\{\mathrm{TpW}(\mathrm{NO})\left(\mathrm{PMe}_{3}\right)\right\}$

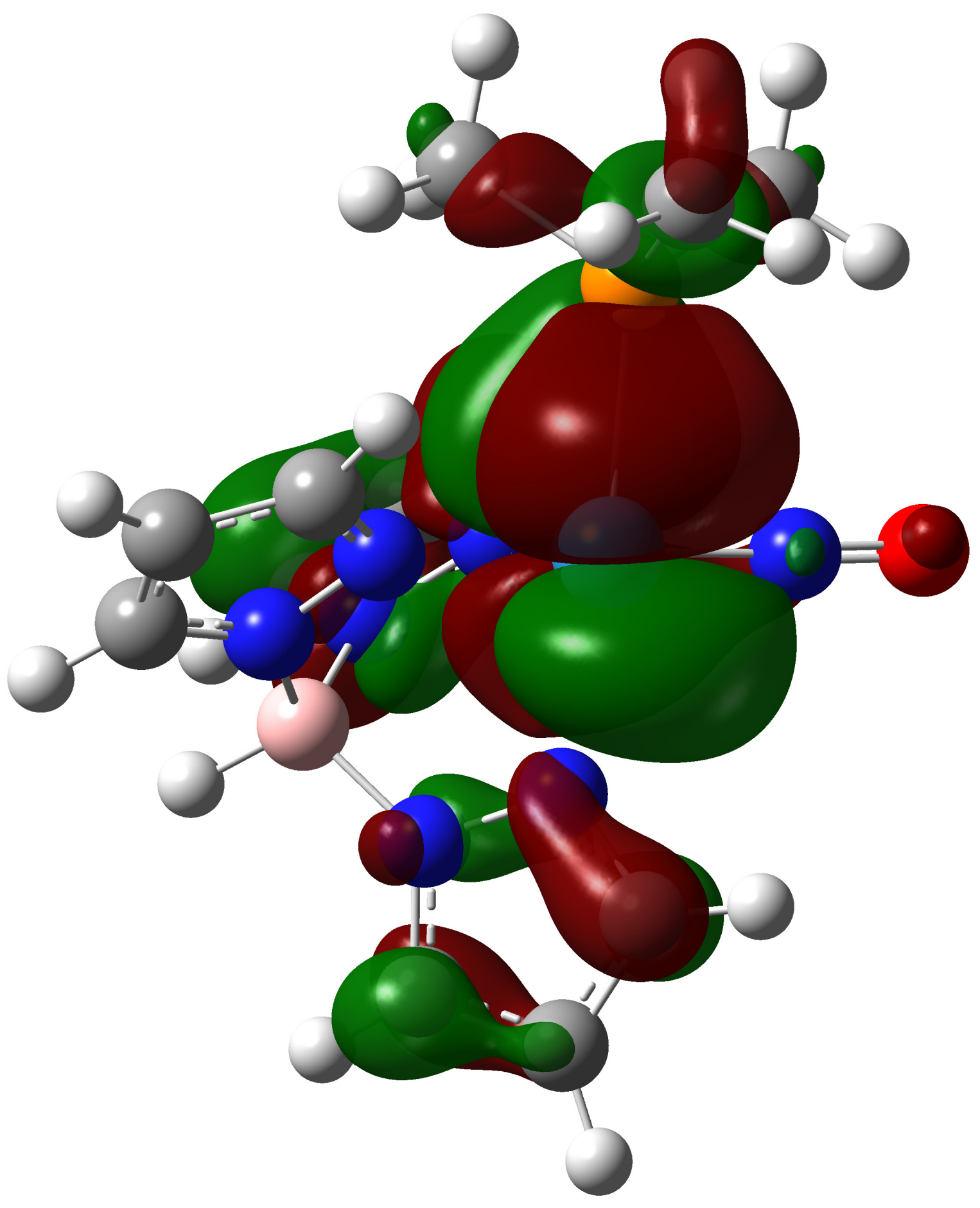




\begin{tabular}{|c|c|c|c|c|c|c|c|}
\hline TITLE & \multicolumn{7}{|c|}{$\left[\left(\mathrm{NH}_{3}\right)_{3} \mathrm{~W}\left(\mathrm{PMe}_{3}\right)(\mathrm{NO})\left(\mathrm{C}_{6} \mathrm{H}_{9}\right)\right]^{2+}$ Distal } \\
\hline REMARK & 1 & Fil & eat & y Gauss & View $5 .($ & 0.8 & \\
\hline HETATM & 1 & W & 0 & -0.156 & -0.647 & -0.021 & W \\
\hline HETATM & 2 & $\mathrm{~N}$ & 0 & -0.611 & 0.036 & 1.57 & $\mathrm{~N}$ \\
\hline HETATM & 3 & $\mathrm{O}$ & 0 & -0.867 & 0.434 & 2.659 & $\mathrm{O}$ \\
\hline HETATM & 4 & $\mathrm{C}$ & 0 & -0.703 & 1.077 & -1.416 & $\mathrm{C}$ \\
\hline HETATM & 5 & $\mathrm{H}$ & 0 & -0.1 & 1.064 & -2.325 & $\mathrm{H}$ \\
\hline HETATM & 6 & $C$ & 0 & -1.799 & 0.131 & -1.418 & $C$ \\
\hline HETATM & 7 & $\mathrm{H}$ & 0 & -1.937 & -0.614 & -2.201 & $\mathrm{H}$ \\
\hline HETATM & 8 & C & 0 & -2.731 & 0.222 & -0.388 & $\mathrm{C}$ \\
\hline HETATM & 9 & $\mathrm{H}$ & 0 & -3.493 & -0.553 & -0.302 & $\mathrm{H}$ \\
\hline HETATM & 10 & $\mathrm{~N}$ & 0 & 1.272 & -2.106 & 1.063 & $\mathrm{~N}$ \\
\hline HETATM & 11 & $\mathrm{H}$ & 0 & 0.88 & -3.039 & 1.215 & $\mathrm{H}$ \\
\hline HETATM & 12 & $\mathrm{H}$ & 0 & 2.191 & -2.264 & 0.647 & $\mathrm{H}$ \\
\hline HETATM & 13 & $\mathrm{~N}$ & 0 & 0.621 & -1.675 & -1.997 & $\mathrm{~N}$ \\
\hline HETATM & 14 & $\mathrm{H}$ & 0 & 1.583 & -2.022 & -1.966 & $\mathrm{H}$ \\
\hline HETATM & 15 & $\mathrm{H}$ & 0 & 0.067 & -2.468 & -2.334 & $\mathrm{H}$ \\
\hline HETATM & 16 & $\mathrm{~N}$ & 0 & -1.485 & -2.551 & 0.174 & $\mathrm{~N}$ \\
\hline HETATM & 17 & $\mathrm{H}$ & 0 & -1.022 & -3.455 & 0.051 & $\mathrm{H}$ \\
\hline HETATM & 18 & $\mathrm{H}$ & 0 & -1.914 & -2.586 & 1.103 & $\mathrm{H}$ \\
\hline HETATM & 19 & $\mathrm{H}$ & 0 & 0.612 & -1.005 & -2.772 & $\mathrm{H}$ \\
\hline HETATM & 20 & $\mathrm{H}$ & 0 & 1.46 & -1.75 & 2.005 & $\mathrm{H}$ \\
\hline HETATM & 21 & $\mathrm{H}$ & 0 & -2.271 & -2.572 & -0.48 & $\mathrm{H}$ \\
\hline HETATM & 22 & C & 0 & -1.057 & 2.495 & -0.958 & $\mathrm{C}$ \\
\hline HETATM & 23 & C & 0 & -2.977 & 1.459 & 0.413 & $C$ \\
\hline HETATM & 24 & $\mathrm{H}$ & 0 & -3.214 & 1.21 & 1.455 & $\mathrm{H}$ \\
\hline HETATM & 25 & $\mathrm{H}$ & 0 & -3.931 & 1.833 & -0.001 & $\mathrm{H}$ \\
\hline HETATM & 26 & C & 0 & -1.881 & 2.54 & 0.338 & $C$ \\
\hline HETATM & 27 & $\mathrm{H}$ & 0 & -0.159 & 3.111 & -0.846 & $\mathrm{H}$ \\
\hline HETATM & 28 & $\mathrm{H}$ & 0 & -1.623 & 2.953 & -1.781 & $\mathrm{H}$ \\
\hline HETATM & 29 & $\mathrm{H}$ & 0 & -2.344 & 3.526 & 0.44 & $\mathrm{H}$ \\
\hline HETATM & 30 & $\mathrm{H}$ & 0 & -1.221 & 2.427 & 1.201 & $\mathrm{H}$ \\
\hline HETATM & 31 & $P$ & 0 & 1.994 & 0.823 & 0.01 & $P$ \\
\hline HETATM & 32 & C & 0 & 1.898 & 2.199 & 1.228 & $C$ \\
\hline HETATM & 33 & $\mathrm{H}$ & 0 & 1.657 & 1.807 & 2.22 & $\mathrm{H}$ \\
\hline HETATM & 34 & $\mathrm{H}$ & 0 & 2.863 & 2.715 & 1.273 & $\mathrm{H}$ \\
\hline HETATM & 35 & $\mathrm{H}$ & 0 & 1.128 & 2.919 & 0.947 & $\mathrm{H}$ \\
\hline HETATM & 36 & C & 0 & 3.547 & -0.042 & 0.536 & $\mathrm{C}$ \\
\hline HETATM & 37 & $\mathrm{H}$ & 0 & 3.808 & -0.865 & -0.138 & $\mathrm{H}$ \\
\hline HETATM & 38 & $\mathrm{H}$ & 0 & 4.374 & 0.675 & 0.507 & $\mathrm{H}$ \\
\hline HETATM & 39 & $\mathrm{H}$ & 0 & 3.479 & -0.413 & 1.563 & $\mathrm{H}$ \\
\hline HETATM & 40 & C & 0 & 2.55 & 1.612 & -1.562 & $\mathrm{C}$ \\
\hline HETATM & 41 & $\mathrm{H}$ & 0 & 3.483 & 2.155 & -1.38 & $\mathrm{H}$ \\
\hline HETATM & 42 & $\mathrm{H}$ & 0 & 2.741 & 0.859 & -2.333 & $\mathrm{H}$ \\
\hline
\end{tabular}


S118

$\begin{array}{lllllll}\text { HETATM } & 43 & \text { H } & 0 & 1.808 & 2.32 & -1.936 \text { H } \\ \text { END } & & & & & & \\ \text { CONECT } & 1 & 2 & & & & \\ \text { CONECT } & 2 & 1 & 3 & & & \\ \text { CONECT } & 3 & 2 & & & & \\ \text { CONECT } & 4 & 5 & 6 & 22 & \\ \text { CONECT } & 5 & 4 & & & \\ \text { CONECT } & 6 & 4 & 7 & 8 & \\ \text { CONECT } & 7 & 6 & & & \\ \text { CONECT } & 8 & 6 & 9 & 23 & \\ \text { CONECT } & 9 & 8 & & & \\ \text { CONECT } & 10 & 11 & 12 & 20 & \\ \text { CONECT } & 11 & 10 & & & \\ \text { CONECT } & 12 & 10 & & & \\ \text { CONECT } & 13 & 14 & 15 & 19 & \\ \text { CONECT } & 14 & 13 & & & \\ \text { CONECT } & 15 & 13 & & & \\ \text { CONECT } & 16 & 17 & 18 & 21 & \\ \text { CONECT } & 17 & 16 & & & \\ \text { CONECT } & 18 & 16 & & & \\ \text { CONECT } & 19 & 13 & & & \\ \text { CONECT } & 20 & 10 & & & \\ \text { CONECT } & 21 & 16 & & & \\ \text { CONECT } & 22 & 4 & 26 & 27 & 28 \\ \text { CONECT } & 23 & 8 & 24 & 25 & 26 \\ \text { CONECT } & 24 & 23 & & & \\ \text { CONECT } & 25 & 23 & & & \\ \text { CONECT } & 26 & 22 & 23 & 29 & 30 \\ \text { CONECT } & 27 & 22 & & & \\ \text { CONECT } & 28 & 22 & & & \\ \text { CONECT } & 29 & 26 & & & \\ \text { CONECT } & 30 & 26 & & & \\ \text { CONECT } & 31 & 32 & 36 & 40 & \\ \text { CONECT } & 32 & 31 & 33 & 34 & 35 \\ \text { CONECT } & 33 & 32 & & & \\ \text { CONECT } & 34 & 32 & & & \\ \text { CONECT } & 35 & 32 & & & \\ \text { CONECT } & 36 & 31 & 37 & 38 & 39 \\ \text { CONECT } & 37 & 36 & & & \\ \text { CONECT } & 38 & 36 & & & \\ \text { CONECT } & 39 & 36 & & & \\ \text { CONECT } & 40 & 31 & 41 & 42 & 43 \\ \text { CONECT } & 41 & 40 & & & \\ \text { CONECT } & 42 & 40 & & & & \\ & & & & & \end{array}$


$\begin{array}{lll}\text { CONECT } & 43 \quad 40\end{array}$

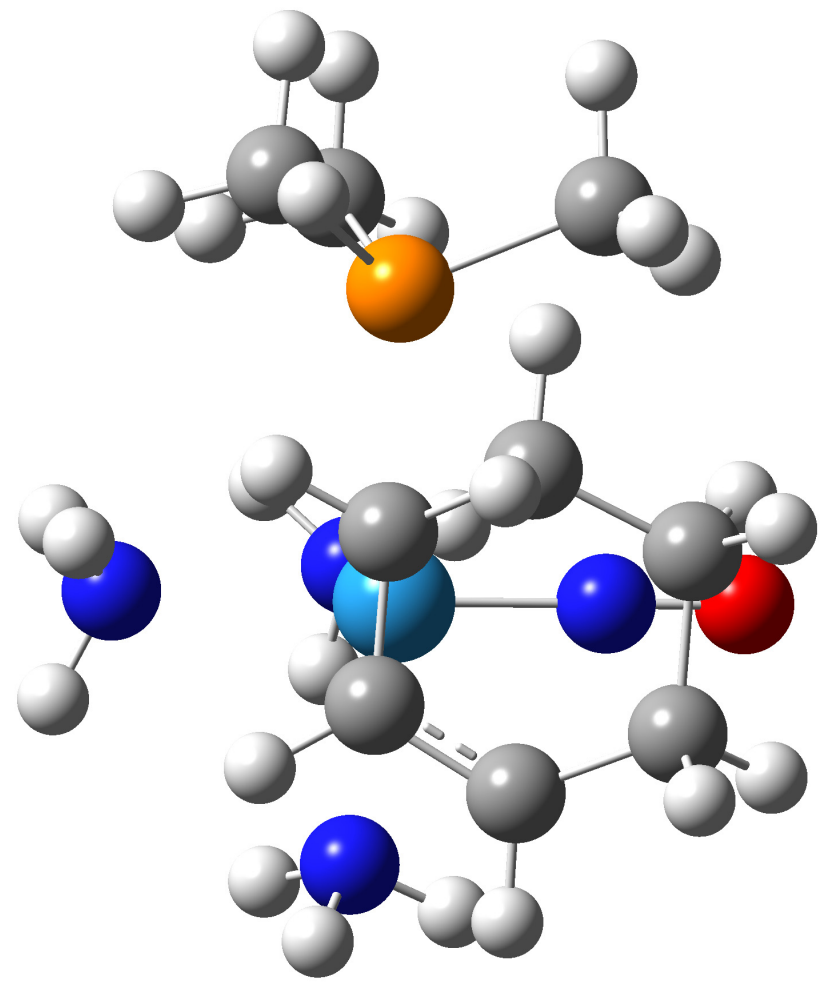




\begin{tabular}{|c|c|c|c|c|c|c|c|}
\hline TITLE & \multicolumn{7}{|c|}{$\left[\left(\mathrm{NH}_{3}\right)_{3} \mathrm{~W}\left(\mathrm{PMe}_{3}\right)(\mathrm{NO})\left(\mathrm{C}_{6} \mathrm{H}_{9}\right)\right]^{2+}$ Proximal } \\
\hline REMARK & 1 & File & & y Gauss & View 5.0 & & \\
\hline HETATM & 1 & W & 0 & -0.105 & -0.569 & 0.019 & \\
\hline HETATM & 2 & $\mathrm{~N}$ & 0 & -0.559 & 0.227 & 1.555 & \\
\hline HETATM & 3 & $\mathrm{O}$ & 0 & -0.809 & 0.713 & 2.61 & \\
\hline HETATM & 4 & $\mathrm{C}$ & 0 & -0.872 & 2.039 & -0.754 & \\
\hline HETATM & 5 & C & 0 & -0.922 & 0.923 & -1.578 & \\
\hline HETATM & 6 & $\mathrm{C}$ & 0 & -1.892 & -0.102 & -1.269 & \\
\hline HETATM & 7 & $\mathrm{C}$ & 0 & -3.23 & 0.367 & -0.696 & ; \\
\hline HETATM & 8 & C & 0 & -3.101 & 1.438 & 0.401 & \\
\hline HETATM & 9 & $C$ & 0 & -1.987 & 2.473 & 0.139 & \\
\hline HETATM & 10 & $\mathrm{~N}$ & 0 & -1.593 & -2.285 & 0.438 & \\
\hline HETATM & 11 & $\mathrm{~N}$ & 0 & 1.133 & -2.137 & 1.162 & \\
\hline HETATM & 12 & $C$ & 0 & 3.655 & -0.177 & 0.375 & \\
\hline HETATM & 13 & $P$ & 0 & 2.139 & 0.788 & -0.075 & \\
\hline HETATM & 14 & C & 0 & 2.21 & 2.194 & 1.113 & \\
\hline HETATM & 15 & $C$ & 0 & 2.62 & 1.507 & -1.705 & \\
\hline HETATM & 16 & $\mathrm{~N}$ & 0 & 0.58 & -1.813 & -1.879 & \\
\hline HETATM & 17 & $\mathrm{H}$ & 0 & -0.086 & 2.773 & -0.925 & \\
\hline HETATM & 18 & $\mathrm{H}$ & 0 & -0.259 & 0.876 & -2.439 & \\
\hline HETATM & 19 & $\mathrm{H}$ & 0 & -2.005 & -0.864 & -2.045 & \\
\hline HETATM & 20 & $\mathrm{H}$ & 0 & -3.814 & 0.755 & -1.542 & \\
\hline HETATM & 21 & $\mathrm{H}$ & 0 & -3.818 & -0.479 & -0.316 & \\
\hline HETATM & 22 & $\mathrm{H}$ & 0 & -2.917 & 0.961 & 1.366 & \\
\hline HETATM & 23 & $\mathrm{H}$ & 0 & -4.055 & 1.962 & 0.508 & \\
\hline HETATM & 24 & $\mathrm{H}$ & 0 & -2.388 & 3.362 & -0.382 & \\
\hline HETATM & 25 & $\mathrm{H}$ & 0 & -1.595 & 2.872 & 1.084 & \\
\hline HETATM & 26 & $\mathrm{H}$ & 0 & 1.494 & 2.973 & 0.841 & \\
\hline HETATM & 27 & $\mathrm{H}$ & 0 & 1.965 & 1.845 & 2.121 & $\mathrm{H}$ \\
\hline HETATM & 28 & $\mathrm{H}$ & 0 & 3.215 & 2.628 & 1.119 & \\
\hline HETATM & 29 & $\mathrm{H}$ & 0 & 3.782 & -1.064 & -0.254 & $t$ \\
\hline HETATM & 30 & $\mathrm{H}$ & 0 & 4.537 & 0.456 & 0.232 & 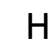 \\
\hline HETATM & 31 & $\mathrm{H}$ & 0 & 3.643 & -0.468 & 1.432 & \\
\hline HETATM & 32 & $\mathrm{H}$ & 0 & 3.55 & 2.075 & -1.599 & \\
\hline HETATM & 33 & $\mathrm{H}$ & 0 & 2.785 & 0.715 & -2.442 & \\
\hline HETATM & 34 & $\mathrm{H}$ & 0 & 1.85 & 2.183 & -2.088 & \\
\hline HETATM & 35 & $\mathrm{H}$ & 0 & 0.416 & -1.297 & -2.747 & 7 \\
\hline HETATM & 36 & $\mathrm{H}$ & 0 & 1.572 & -2.061 & -1.907 & 7 \\
\hline HETATM & 37 & $\mathrm{H}$ & 0 & 0.084 & -2.699 & -2.013 & \\
\hline HETATM & 38 & $\mathrm{H}$ & 0 & 1.045 & -3.106 & 0.845 & \\
\hline HETATM & 39 & $\mathrm{H}$ & 0 & 2.136 & -1.944 & 1.156 & $\Pi$ \\
\hline HETATM & 40 & $\mathrm{H}$ & 0 & 0.87 & -2.139 & 2.152 & \\
\hline HETATM & 41 & $\mathrm{H}$ & 0 & -1.829 & -2.897 & -0.347 & \\
\hline HETATM & 42 & $\mathrm{H}$ & 0 & -1.377 & -2.906 & 1.222 & \\
\hline
\end{tabular}




$\begin{array}{lllllll}\text { HETATM } & 43 & \text { H } & 0 & -2.476 & -1.8360 .693 \\ \text { END } & & & & & \\ \text { CONECT } & 1 & 2 & & & \\ \text { CONECT } & 2 & 1 & 3 & & \\ \text { CONECT } & 3 & 2 & & & \\ \text { CONECT } & 4 & 5 & 9 & 17 & \\ \text { CONECT } & 5 & 4 & 6 & 18 & \\ \text { CONECT } & 6 & 5 & 7 & 19 & \\ \text { CONECT } & 7 & 6 & 8 & 20 & 21 \\ \text { CONECT } & 8 & 7 & 9 & 22 & 23 \\ \text { CONECT } & 9 & 8 & 4 & 24 & 25 \\ \text { CONECT } & 10 & 41 & 42 & 43 & \\ \text { CONECT } & 11 & 38 & 39 & 40 & \\ \text { CONECT } & 12 & 13 & 29 & 30 & 31 \\ \text { CONECT } & 13 & 12 & 14 & 15 & \\ \text { CONECT } & 14 & 13 & 26 & 27 & 28 \\ \text { CONECT } & 15 & 13 & 32 & 33 & 34 \\ \text { CONECT } & 16 & 35 & 36 & 37 & \\ \text { CONECT } & 17 & 4 & & & \\ \text { CONECT } & 18 & 5 & & & \\ \text { CONECT } & 19 & 6 & & & \\ \text { CONECT } & 20 & 7 & & & \\ \text { CONECT } & 21 & 7 & & & \\ \text { CONECT } & 22 & 8 & & & \\ \text { CONECT } & 23 & 8 & & & \\ \text { CONECT } & 24 & 9 & & & \\ \text { CONECT } & 25 & 9 & & & \\ \text { CONECT } & 26 & 14 & & & \\ \text { CONECT } & 27 & 14 & & & \\ \text { CONECT } & 28 & 14 & & & \\ \text { CONECT } & 29 & 12 & & & \\ \text { CONECT } & 30 & 12 & & & \\ \text { CONECT } & 31 & 12 & & & \\ \text { CONECT } & 32 & 15 & & & \\ \text { CONECT } & 33 & 15 & & & \\ \text { CONECT } & 34 & 15 & & & \\ \text { CONECT } & 35 & 16 & & & \\ \text { CONECT } & 36 & 16 & & & \\ \text { CONECT } & 37 & 16 & & & \\ \text { CONECT } & 38 & 11 & & & \\ \text { CONECT } & 39 & 11 & & & \\ \text { CONECT } & 40 & 11 & & & \\ \text { CONECT } & 41 & 10 & & & \\ \text { CONECT } & 42 & 10 & & & \\ & & & & & \end{array}$


$\begin{array}{lll}\text { CONECT } & 43 \quad 10\end{array}$
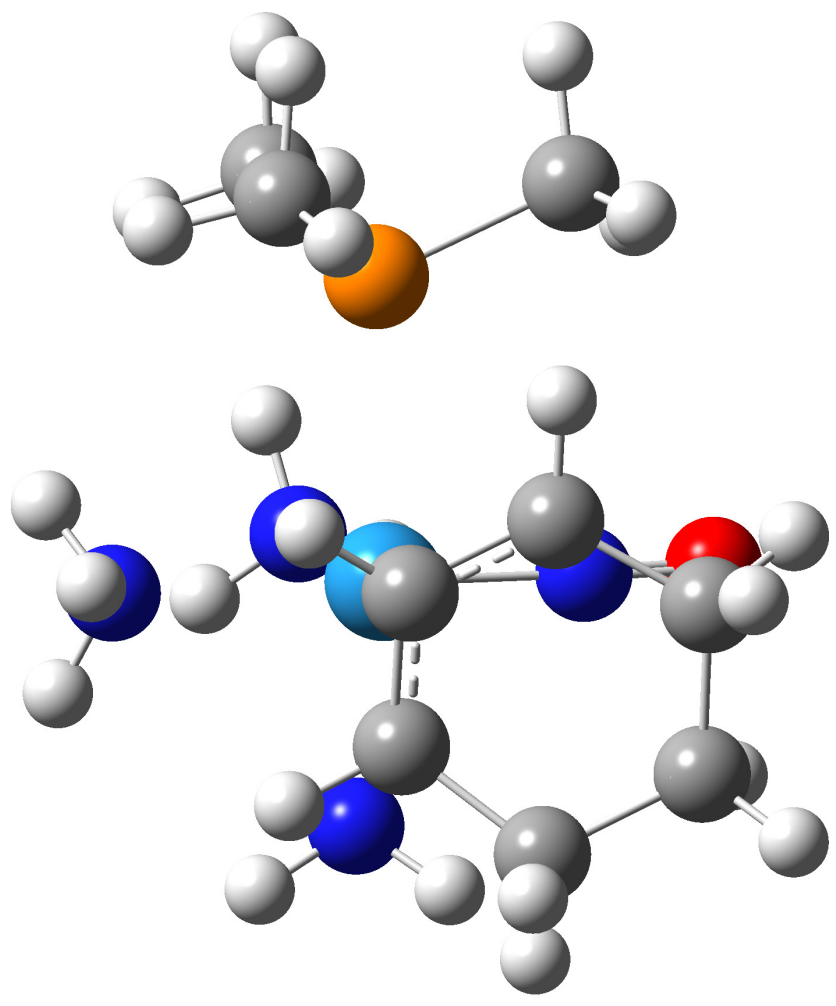


\begin{tabular}{|c|c|c|c|c|c|c|c|}
\hline TITLE & {$[(\mathbf{N}$} & & & $\left.\left(\mathrm{C}_{6} \mathrm{H}_{9}\right)\right]^{2}$ & ${ }^{++}$Transi & ition Sta & ate \\
\hline REMARK & 1 & & & y Gauss & View 5. & 0.8 & \\
\hline HETATM & 1 & W & 0 & -0.144 & -0.587 & -0.022 & \\
\hline HETATM & 2 & $\mathrm{~N}$ & 0 & -0.57 & 0.068 & 1.587 & \\
\hline HETATM & 3 & 0 & 0 & -0.749 & 0.377 & 2.718 & \\
\hline HETATM & 4 & $C$ & 0 & -0.648 & 1.553 & -1.146 & \\
\hline HETATM & 5 & C & 0 & -1.408 & 0.487 & -1.678 & \\
\hline HETATM & 6 & C & 0 & -2.466 & -0.011 & -0.916 & \\
\hline HETATM & 7 & C & 0 & -3.273 & 0.867 & 0.009 & \\
\hline HETATM & 8 & C & 0 & -2.536 & 2.108 & 0.553 & \\
\hline HETATM & 9 & C & 0 & -1.375 & 2.614 & -0.328 & \\
\hline HETATM & 10 & $N$ & 0 & -1.533 & -2.433 & 0.254 & \\
\hline HETATM & 11 & $\mathrm{~N}$ & 0 & 0.667 & -1.713 & -1.941 & \\
\hline HETATM & 12 & $N$ & 0 & 1.156 & -2.113 & 1.104 & \\
\hline HETATM & 13 & C & 0 & 2.864 & 1.099 & -1.665 & \\
\hline HETATM & 14 & $P$ & 0 & 2.069 & 0.802 & -0.023 & \\
\hline HETATM & 15 & C & 0 & 1.979 & 2.462 & 0.765 & \\
\hline HETATM & 16 & C & 0 & 3.477 & 0.035 & 0.91 & \\
\hline HETATM & 17 & $\mathrm{H}$ & 0 & 0.145 & 1.932 & -1.784 & \\
\hline HETATM & 18 & $\mathrm{H}$ & 0 & -1.134 & 0.012 & -2.617 & \\
\hline HETATM & 19 & $\mathrm{H}$ & 0 & -2.985 & -0.887 & -1.305 & \\
\hline HETATM & 20 & $\mathrm{H}$ & 0 & -3.704 & 0.288 & 0.834 & \\
\hline HETATM & 21 & $\mathrm{H}$ & 0 & -4.139 & 1.168 & -0.604 & \\
\hline HETATM & 22 & $\mathrm{H}$ & 0 & -2.163 & 1.893 & 1.554 & \\
\hline HETATM & 23 & $\mathrm{H}$ & 0 & -3.258 & 2.92 & 0.679 & \\
\hline HETATM & 24 & $\mathrm{H}$ & 0 & -1.754 & 3.319 & -1.084 & \\
\hline HETATM & 25 & $\mathrm{H}$ & 0 & -0.683 & 3.193 & 0.288 & \\
\hline HETATM & 26 & $\mathrm{H}$ & 0 & 1.448 & 3.169 & 0.125 & \\
\hline HETATM & 27 & $\mathrm{H}$ & 0 & 1.462 & 2.398 & 1.727 & \\
\hline HETATM & 28 & $\mathrm{H}$ & 0 & 2.993 & 2.839 & 0.93 & \\
\hline HETATM & 29 & $\mathrm{H}$ & 0 & 3.802 & -0.915 & 0.469 & \\
\hline HETATM & 30 & $\mathrm{H}$ & 0 & 4.335 & 0.713 & 0.864 & \\
\hline HETATM & 31 & $\mathrm{H}$ & 0 & 3.231 & -0.102 & 1.968 & \\
\hline HETATM & 32 & $\mathrm{H}$ & 0 & 3.746 & 1.735 & -1.534 & \\
\hline HETATM & 33 & $\mathrm{H}$ & 0 & 3.201 & 0.155 & -2.108 & \\
\hline HETATM & 34 & $\mathrm{H}$ & 0 & 2.188 & 1.6 & -2.364 & \\
\hline HETATM & 35 & $\mathrm{H}$ & 0 & 1.04 & -1.05 & -2.626 & \\
\hline HETATM & 36 & $\mathrm{H}$ & 0 & 1.434 & -2.369 & -1.774 & \\
\hline HETATM & 37 & $\mathrm{H}$ & 0 & -0.034 & -2.253 & -2.457 & \\
\hline HETATM & 38 & $\mathrm{H}$ & 0 & 2.129 & -1.829 & 1.231 & \\
\hline HETATM & 39 & $\mathrm{H}$ & 0 & 1.201 & -3.058 & 0.713 & \\
\hline HETATM & 40 & $\mathrm{H}$ & 0 & 0.794 & -2.219 & 2.057 & \\
\hline HETATM & 41 & $\mathrm{H}$ & 0 & -1.148 & -3.205 & 0.805 & \\
\hline HETATM & 42 & $\mathrm{H}$ & 0 & -2.364 & -2.133 & 0.771 & \\
\hline
\end{tabular}




$\begin{array}{lllllll}\text { HETATM } & 43 & \text { H } & 0 & -1.893 & -2.867-0.6 & \text { H } \\ \text { END } & & & & & \\ \text { CONECT } & 1 & 2 & & & \\ \text { CONECT } & 2 & 1 & 3 & & \\ \text { CONECT } & 3 & 2 & & & \\ \text { CONECT } & 4 & 5 & 9 & 17 & \\ \text { CONECT } & 5 & 4 & 6 & 18 & \\ \text { CONECT } & 6 & 5 & 7 & 19 & \\ \text { CONECT } & 7 & 6 & 8 & 20 & 21 \\ \text { CONECT } & 8 & 7 & 9 & 22 & 23 \\ \text { CONECT } & 9 & 8 & 4 & 24 & 25 \\ \text { CONECT } & 10 & 41 & 42 & 43 & \\ \text { CONECT } & 11 & 35 & 36 & 37 & \\ \text { CONECT } & 12 & 38 & 39 & 40 & \\ \text { CONECT } & 13 & 14 & 32 & 33 & 34 \\ \text { CONECT } & 14 & 13 & 15 & 16 & \\ \text { CONECT } & 15 & 14 & 26 & 27 & 28 \\ \text { CONECT } & 16 & 14 & 29 & 30 & 31 \\ \text { CONECT } & 17 & 4 & & & \\ \text { CONECT } & 18 & 5 & & & \\ \text { CONECT } & 19 & 6 & & & \\ \text { CONECT } & 20 & 7 & & & \\ \text { CONECT } & 21 & 7 & & & \\ \text { CONECT } & 22 & 8 & & & \\ \text { CONECT } & 23 & 8 & & & \\ \text { CONECT } & 24 & 9 & & & \\ \text { CONECT } & 25 & 9 & & & \\ \text { CONECT } & 26 & 15 & & & \\ \text { CONECT } & 27 & 15 & & & \\ \text { CONECT } & 28 & 15 & & & \\ \text { CONECT } & 29 & 16 & & & \\ \text { CONECT } & 30 & 16 & & & \\ \text { CONECT } & 31 & 16 & & & \\ \text { CONECT } & 32 & 13 & & & \\ \text { CONECT } & 33 & 13 & & & \\ \text { CONECT } & 34 & 13 & & & \\ \text { CONECT } & 35 & 11 & & & \\ \text { CONECT } & 36 & 11 & & & \\ \text { CONECT } & 37 & 11 & & & \\ \text { CONECT } & 38 & 12 & & & \\ \text { CONECT } & 39 & 12 & & & \\ \text { CONECT } & 40 & 12 & & & \\ \text { CONECT } & 41 & 10 & & & \\ \text { CONECT } & 42 & 10 & & & \\ & & & & & \end{array}$


$\begin{array}{lll}\text { CONECT } & 43 \quad 10\end{array}$

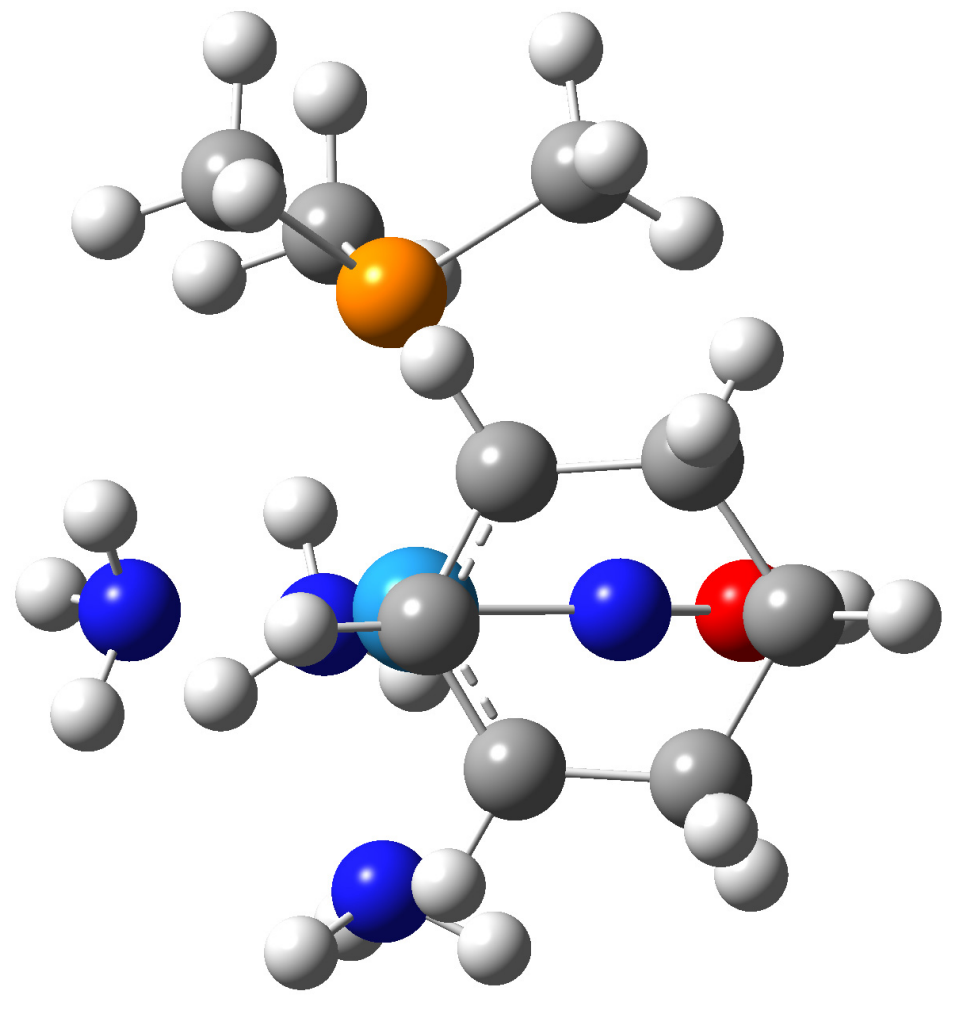




\section{TITLE $\quad\left[\mathrm{TpW}(\mathrm{NO})\left(\mathrm{NH}_{3}\right)\left(\mathrm{C}_{6} \mathrm{H}_{9}\right)\right]^{+}$Distal}

\begin{tabular}{|c|c|c|c|c|c|c|c|}
\hline REMARK & 1 & \multicolumn{6}{|c|}{ File created by GaussView 5.0.8 } \\
\hline HETATM & 1 & B & 0 & -2.749 & 0.147 & 0.593 & \\
\hline HETATM & 2 & $\mathrm{~N}$ & 0 & -2.583 & -0.493 & -0.811 & \\
\hline HETATM & 3 & $\mathrm{~N}$ & 0 & -1.935 & -0.732 & 1.575 & \\
\hline HETATM & 4 & $\mathrm{~N}$ & 0 & -2.166 & 1.571 & 0.562 & \\
\hline HETATM & 5 & $\mathrm{H}$ & 0 & -3.901 & 0.173 & 0.91 & \\
\hline HETATM & 6 & $\mathrm{~N}$ & 0 & -1.334 & -0.711 & -1.309 & \\
\hline HETATM & 7 & C & 0 & -1.478 & -1.465 & -2.418 & \\
\hline HETATM & 8 & C & 0 & -2.829 & -1.724 & -2.65 & \\
\hline HETATM & 9 & C & 0 & -3.492 & -1.098 & -1.6 & \\
\hline HETATM & 10 & C & 0 & -2.774 & 2.747 & 0.812 & \\
\hline HETATM & 11 & C & 0 & -1.846 & 3.769 & 0.652 & \\
\hline HETATM & 12 & C & 0 & -0.665 & 3.115 & 0.297 & \\
\hline HETATM & 13 & $N$ & 0 & -0.857 & 1.78 & 0.241 & \\
\hline HETATM & 14 & C & 0 & -2.413 & -1.54 & 2.544 & \\
\hline HETATM & 15 & C & 0 & -1.39 & -2.369 & 2.984 & \\
\hline HETATM & 16 & C & 0 & -0.288 & -2.009 & 2.208 & \\
\hline HETATM & 17 & $N$ & 0 & -0.611 & -1.011 & 1.364 & \\
\hline HETATM & 18 & $\mathrm{H}$ & 0 & -0.608 & -1.803 & -2.966 & \\
\hline HETATM & 19 & $\mathrm{H}$ & 0 & -3.263 & -2.299 & -3.454 & \\
\hline HETATM & 20 & $\mathrm{H}$ & 0 & -4.544 & -1.052 & -1.357 & \\
\hline HETATM & 21 & $\mathrm{H}$ & 0 & -3.818 & 2.776 & 1.087 & \\
\hline HETATM & 22 & $\mathrm{H}$ & 0 & -2.003 & 4.829 & 0.779 & \\
\hline HETATM & 23 & $\mathrm{H}$ & 0 & 0.308 & 3.542 & 0.1 & \\
\hline HETATM & 24 & $\mathrm{H}$ & 0 & -3.448 & -1.474 & 2.845 & \\
\hline HETATM & 25 & $\mathrm{H}$ & 0 & -1.438 & -3.127 & 3.751 & \\
\hline HETATM & 26 & $\mathrm{H}$ & 0 & 0.709 & -2.423 & 2.208 & \\
\hline HETATM & 27 & W & 0 & 0.462 & 0.005 & -0.273 & \\
\hline HETATM & 28 & $\mathrm{~N}$ & 0 & 1.4 & -1.401 & -0.849 & \\
\hline HETATM & 29 & $\mathrm{O}$ & 0 & 2.022 & -2.325 & -1.278 & \\
\hline HETATM & 30 & C & 0 & 2.351 & -0.187 & 1.67 & \\
\hline HETATM & 31 & C & 0 & 1.935 & 1.056 & 1.22 & \\
\hline HETATM & 32 & C & 0 & 3.593 & -0.849 & 1.129 & \\
\hline HETATM & 33 & $\mathrm{H}$ & 0 & 3.371 & -1.76 & 0.56 & \\
\hline HETATM & 34 & $\mathrm{H}$ & 0 & 1.284 & 1.665 & 1.84 & \\
\hline HETATM & 35 & C & 0 & 4.414 & 0.144 & 0.285 & $C$ \\
\hline HETATM & 36 & $\mathrm{H}$ & 0 & 5.234 & -0.381 & -0.216 & \\
\hline HETATM & 37 & C & 0 & 3.563 & 0.906 & -0.747 & 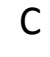 \\
\hline HETATM & 38 & C & 0 & 2.251 & 1.425 & -0.133 & 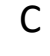 \\
\hline HETATM & 39 & $\mathrm{H}$ & 0 & 2.002 & 2.451 & -0.404 & \\
\hline HETATM & 40 & $\mathrm{H}$ & 0 & 4.87 & 0.875 & 0.964 & \\
\hline HETATM & 41 & $\mathrm{H}$ & 0 & 4.182 & -1.183 & 1.993 & 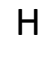 \\
\hline HETATM & 42 & $\mathrm{H}$ & 0 & 1.941 & -0.555 & 2.606 & \\
\hline
\end{tabular}




\begin{tabular}{|c|c|c|c|c|c|c|}
\hline HETATM & 43 & $\mathrm{H}$ & 0 & 4.142 & 1.758 & $-1.122 \mathrm{H}$ \\
\hline HETATM & 44 & $\mathrm{H}$ & 0 & 3.377 & 0.258 & $-1.615 \mathrm{H}$ \\
\hline HETATM & 45 & $\mathrm{~N}$ & 0 & 0.594 & 1.018 & $-2.297 \mathrm{~N}$ \\
\hline HETATM & 46 & H & 0 & 0.294 & 1.992 & $-2.237 \mathrm{H}$ \\
\hline HETATM & 47 & $\mathrm{H}$ & 0 & 1.539 & 1.014 & $-2.684 \mathrm{H}$ \\
\hline HETATM & 48 & $\mathrm{H}$ & 0 & -0.019 & 0.569 & $-2.98 \mathrm{H}$ \\
\hline \multicolumn{7}{|l|}{ END } \\
\hline CONECT & 1 & 2 & 3 & 4 & 5 & \\
\hline CONECT & 2 & 1 & 6 & 9 & & \\
\hline CONECT & 3 & 1 & 14 & 17 & & \\
\hline CONECT & 4 & 1 & 10 & 13 & & \\
\hline CONECT & 5 & 1 & & & & \\
\hline CONECT & 6 & 2 & 7 & & & \\
\hline CONECT & 7 & 6 & 8 & 18 & & \\
\hline CONECT & 8 & 7 & 9 & 19 & & \\
\hline CONECT & 9 & 2 & 8 & 20 & & \\
\hline CONECT & 10 & 4 & 11 & 21 & & \\
\hline CONECT & 11 & 10 & 12 & 22 & & \\
\hline CONECT & 12 & 11 & 13 & 23 & & \\
\hline CONECT & 13 & 4 & 12 & & & \\
\hline CONECT & 14 & 3 & 15 & 24 & & \\
\hline CONECT & 15 & 14 & 16 & 25 & & \\
\hline CONECT & 16 & 15 & 17 & 26 & & \\
\hline CONECT & 17 & 3 & 16 & & & \\
\hline CONECT & 18 & 7 & & & & \\
\hline CONECT & 19 & 8 & & & & \\
\hline CONECT & 20 & 9 & & & & \\
\hline CONECT & 21 & 10 & & & & \\
\hline CONECT & 22 & 11 & & & & \\
\hline CONECT & 23 & 12 & & & & \\
\hline CONECT & 24 & 14 & & & & \\
\hline CONECT & 25 & 15 & & & & \\
\hline CONECT & 26 & 16 & & & & \\
\hline CONECT & 27 & 28 & & & & \\
\hline CONECT & 28 & 27 & 29 & & & \\
\hline CONECT & 29 & 28 & & & & \\
\hline CONECT & 30 & 31 & 32 & 42 & & \\
\hline CONECT & 31 & 30 & 34 & 38 & & \\
\hline CONECT & 32 & 30 & 33 & 35 & 41 & \\
\hline CONECT & 33 & 32 & & & & \\
\hline CONECT & 34 & 31 & & & & \\
\hline CONECT & 35 & 32 & 36 & 37 & 40 & \\
\hline CONECT & 36 & 35 & & & & \\
\hline CONECT & 37 & 35 & 38 & 43 & 44 & \\
\hline
\end{tabular}




$\begin{array}{lllll}\text { CONECT } & 38 & 37 & 31 & 39 \\ \text { CONECT } & 39 & 38 & & \\ \text { CONECT } & 40 & 35 & & \\ \text { CONECT } & 41 & 32 & & \\ \text { CONECT } & 42 & 30 & & \\ \text { CONECT } & 43 & 37 & & \\ \text { CONECT } & 44 & 37 & & \\ \text { CONECT } & 45 & 46 & 47 & 48 \\ \text { CONECT } & 46 & 45 & & \\ \text { CONECT } & 47 & 45 & & \\ \text { CONECT } & 48 & 45 & & \end{array}$

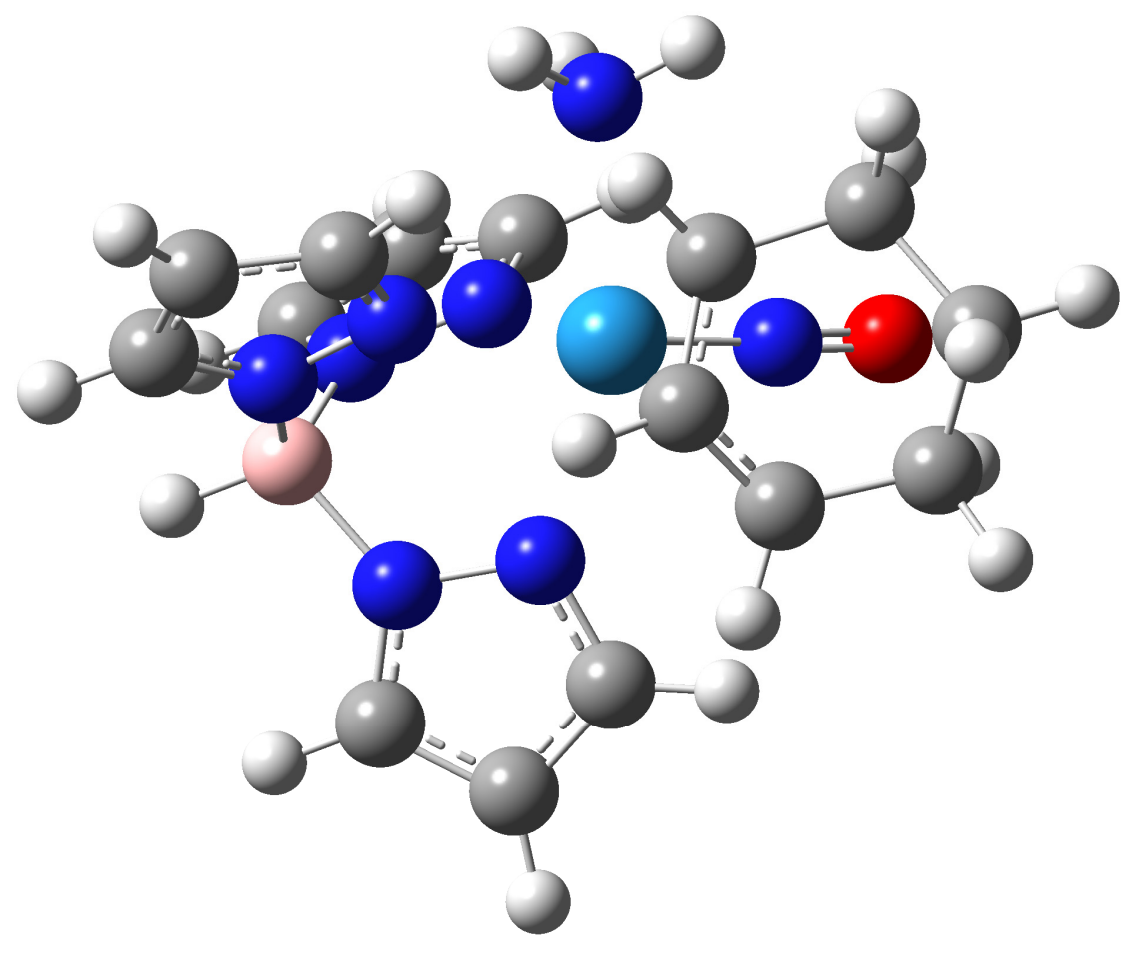




\begin{tabular}{|c|c|c|c|c|c|c|c|}
\hline \multirow{3}{*}{$\begin{array}{l}\text { TITLE } \\
\text { REMARK } \\
\text { HETATM }\end{array}$} & \multicolumn{7}{|c|}{$\left[\mathrm{TpW}(\mathrm{NO})\left(\mathrm{NH}_{3}\right)\left(\mathrm{C}_{6} \mathrm{H}_{9}\right)\right]^{+}$Proximal } \\
\hline & \multirow{2}{*}{$\begin{array}{l}1 \\
1\end{array}$} & \multicolumn{6}{|c|}{ File created by GaussView 5.0 .8} \\
\hline & & W & 0 & 0.479 & 0.14 & -0.352 & W \\
\hline HETATM & 2 & $\mathrm{~N}$ & 0 & 1.434 & -1.208 & -1.038 & $\mathrm{~N}$ \\
\hline HETATM & 3 & 0 & 0 & 1.983 & -2.129 & -1.563 & $\mathrm{O}$ \\
\hline HETATM & 4 & $\mathrm{~N}$ & 0 & -0.377 & -1.108 & 1.249 & $\mathrm{~N}$ \\
\hline HETATM & 5 & $\mathrm{~N}$ & 0 & -1.685 & -0.983 & 1.619 & $\mathrm{~N}$ \\
\hline HETATM & 6 & $\mathrm{~N}$ & 0 & -1.334 & -0.734 & -1.268 & $\mathrm{~N}$ \\
\hline HETATM & 7 & $\mathrm{~N}$ & 0 & -2.558 & -0.681 & -0.676 & $\mathrm{~N}$ \\
\hline HETATM & 8 & $\mathrm{~N}$ & 0 & -1.007 & 1.721 & 0.335 & $\mathrm{~N}$ \\
\hline HETATM & 9 & $\mathrm{~N}$ & 0 & -2.241 & 1.336 & 0.776 & $\mathrm{~N}$ \\
\hline HETATM & 10 & B & 0 & -2.669 & -0.145 & 0.776 & B \\
\hline HETATM & 11 & $\mathrm{H}$ & 0 & -3.787 & -0.255 & 1.186 & $\mathrm{H}$ \\
\hline HETATM & 12 & $C$ & 0 & 0.146 & -2.104 & 1.981 & $C$ \\
\hline HETATM & 13 & $\mathrm{H}$ & 0 & 1.175 & -2.397 & 1.844 & $\mathrm{H}$ \\
\hline HETATM & 14 & C & 0 & -0.82 & -2.626 & 2.847 & C \\
\hline HETATM & 15 & $\mathrm{H}$ & 0 & -0.701 & -3.427 & 3.561 & $\mathrm{H}$ \\
\hline HETATM & 16 & C & 0 & -1.965 & -1.892 & 2.58 & $\mathrm{C}$ \\
\hline HETATM & 17 & $\mathrm{H}$ & 0 & -2.96 & -1.956 & 2.994 & $\mathrm{H}$ \\
\hline HETATM & 18 & $C$ & 0 & -0.995 & 3.069 & 0.396 & $C$ \\
\hline HETATM & 19 & $\mathrm{H}$ & 0 & -0.121 & 3.634 & 0.105 & $\mathrm{H}$ \\
\hline HETATM & 20 & C & 0 & -2.211 & 3.557 & 0.875 & $\mathrm{C}$ \\
\hline HETATM & 21 & $\mathrm{H}$ & 0 & -2.494 & 4.586 & 1.037 & $\mathrm{H}$ \\
\hline HETATM & 22 & $C$ & 0 & -2.972 & 2.419 & 1.104 & $C$ \\
\hline HETATM & 23 & $\mathrm{H}$ & 0 & -3.98 & 2.304 & 1.476 & $\mathrm{H}$ \\
\hline HETATM & 24 & $C$ & 0 & -1.479 & -1.421 & -2.419 & $C$ \\
\hline HETATM & 25 & $\mathrm{H}$ & 0 & -0.622 & -1.63 & -3.044 & $\mathrm{H}$ \\
\hline HETATM & 26 & C & 0 & -2.813 & -1.8 & -2.584 & $C$ \\
\hline HETATM & 27 & $\mathrm{H}$ & 0 & -3.245 & -2.359 & -3.401 & $\mathrm{H}$ \\
\hline HETATM & 28 & C & 0 & -3.458 & -1.32 & -1.449 & $C$ \\
\hline HETATM & 29 & $\mathrm{H}$ & 0 & -4.489 & -1.397 & -1.137 & $\mathrm{H}$ \\
\hline HETATM & 30 & $C$ & 0 & 2.07 & 1.668 & 0.358 & $C$ \\
\hline HETATM & 31 & $\mathrm{H}$ & 0 & 1.627 & 2.656 & 0.289 & $\mathrm{H}$ \\
\hline HETATM & 32 & $C$ & 0 & 2.931 & 1.231 & -0.636 & $\mathrm{C}$ \\
\hline HETATM & 33 & $\mathrm{H}$ & 0 & 3.069 & 1.858 & -1.518 & $\mathrm{H}$ \\
\hline HETATM & 34 & C & 0 & 1.758 & 0.741 & 1.419 & $\mathrm{C}$ \\
\hline HETATM & 35 & $\mathrm{H}$ & 0 & 1.119 & 1.139 & 2.207 & $\mathrm{H}$ \\
\hline HETATM & 36 & $C$ & 0 & 2.929 & -0.097 & 1.931 & C \\
\hline HETATM & 37 & $\mathrm{H}$ & 0 & 2.577 & -0.869 & 2.621 & $\mathrm{H}$ \\
\hline HETATM & 38 & $\mathrm{H}$ & 0 & 3.559 & 0.572 & 2.536 & $\mathrm{H}$ \\
\hline HETATM & 39 & C & 0 & 3.973 & 0.168 & -0.423 & $\mathrm{C}$ \\
\hline HETATM & 40 & $\mathrm{H}$ & 0 & 4.914 & 0.737 & -0.333 & $\mathrm{H}$ \\
\hline HETATM & 41 & $\mathrm{H}$ & 0 & 4.096 & -0.443 & -1.325 & $\mathrm{H}$ \\
\hline
\end{tabular}




\begin{tabular}{|c|c|c|c|c|c|c|}
\hline HETATM & 42 & C & 0 & 3.766 & -0.74 & $0.812 C$ \\
\hline HETATM & 43 & $\mathrm{H}$ & 0 & 4.745 & -1.03 & 1.207 \\
\hline HETATM & 44 & $\mathrm{H}$ & 0 & 3.287 & -1.665 & 0.484 \\
\hline HETATM & 45 & $\mathrm{~N}$ & 0 & 0.363 & 1.326 & $-2.288 N$ \\
\hline HETATM & 46 & $\mathrm{H}$ & 0 & 0.334 & 2.338 & -2.154 \\
\hline HETATM & 47 & $\mathrm{H}$ & 0 & 1.108 & 1.131 & -2.958 \\
\hline HETATM & 48 & $\mathrm{H}$ & 0 & -0.519 & 1.073 & -2.742 \\
\hline \multicolumn{7}{|l|}{ END } \\
\hline CONECT & 1 & 2 & & & & \\
\hline CONECT & 2 & 1 & 3 & & & \\
\hline CONECT & 3 & 2 & & & & \\
\hline CONECT & 4 & 5 & 12 & & & \\
\hline CONECT & 5 & 4 & 10 & 16 & & \\
\hline CONECT & 6 & 7 & 24 & & & \\
\hline CONECT & 7 & 6 & 10 & 28 & & \\
\hline CONECT & 8 & 9 & 18 & & & \\
\hline CONECT & 9 & 8 & 10 & 22 & & \\
\hline CONECT & 10 & 7 & 5 & 9 & 11 & \\
\hline CONECT & 11 & 10 & & & & \\
\hline CONECT & 12 & 4 & 13 & 14 & & \\
\hline CONECT & 13 & 12 & & & & \\
\hline CONECT & 14 & 12 & 15 & 16 & & \\
\hline CONECT & 15 & 14 & & & & \\
\hline CONECT & 16 & 5 & 14 & 17 & & \\
\hline CONECT & 17 & 16 & & & & \\
\hline CONECT & 18 & 8 & 19 & 20 & & \\
\hline CONECT & 19 & 18 & & & & \\
\hline CONECT & 20 & 18 & 21 & 22 & & \\
\hline CONECT & 21 & 20 & & & & \\
\hline CONECT & 22 & 9 & 20 & 23 & & \\
\hline CONECT & 23 & 22 & & & & \\
\hline CONECT & 24 & 6 & 25 & 26 & & \\
\hline CONECT & 25 & 24 & & & & \\
\hline CONECT & 26 & 24 & 27 & 28 & & \\
\hline CONECT & 27 & 26 & & & & \\
\hline CONECT & 28 & 7 & 26 & 29 & & \\
\hline CONECT & 29 & 28 & & & & \\
\hline CONECT & 30 & 31 & 32 & 34 & & \\
\hline CONECT & 31 & 30 & & & & \\
\hline CONECT & 32 & 30 & 33 & 39 & & \\
\hline CONECT & 33 & 32 & & & & \\
\hline CONECT & 34 & 30 & 35 & 36 & & \\
\hline CONECT & 35 & 34 & & & & \\
\hline CONECT & 36 & 34 & 37 & 38 & 42 & \\
\hline
\end{tabular}




$\begin{array}{llllll}\text { CONECT } & 37 & 36 & & & \\ \text { CONECT } & 38 & 36 & & & \\ \text { CONECT } & 39 & 32 & 40 & 41 & 42 \\ \text { CONECT } & 40 & 39 & & & \\ \text { CONECT } & 41 & 39 & & & \\ \text { CONECT } & 42 & 39 & 36 & 43 & 44 \\ \text { CONECT } & 43 & 42 & & & \\ \text { CONECT } & 44 & 42 & & & \\ \text { CONECT } & 45 & 46 & 47 & 48 & \\ \text { CONECT } & 46 & 45 & & & \\ \text { CONECT } & 47 & 45 & & & \\ \text { CONECT } & 48 & 45 & & & \end{array}$

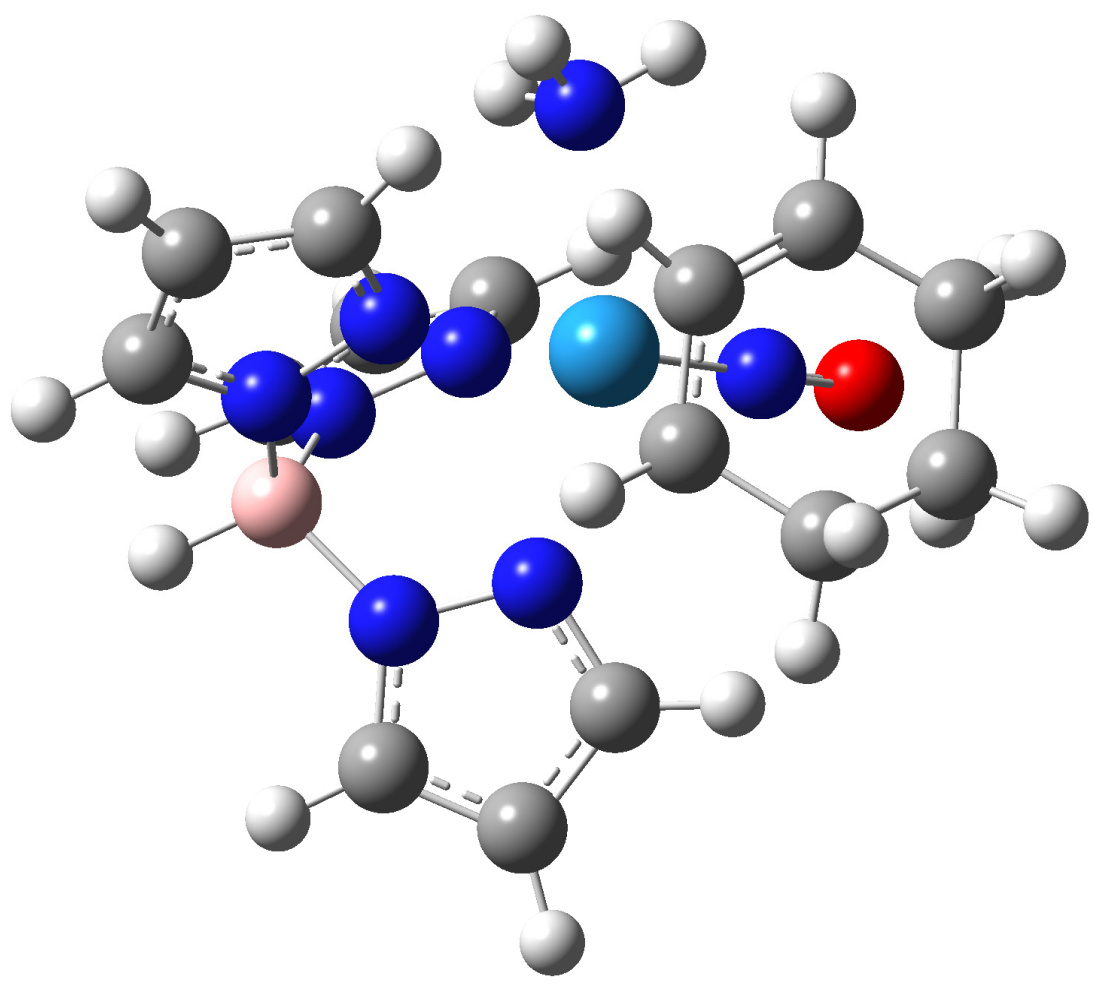


Figure S2: Energy versus reaction coordinate diagrams for distorted allyl isomers and selected transition state structures optimized using B3LYP LANL2DZ and 6-31G(d) hybrid basis set (energies are zero-point corrected)
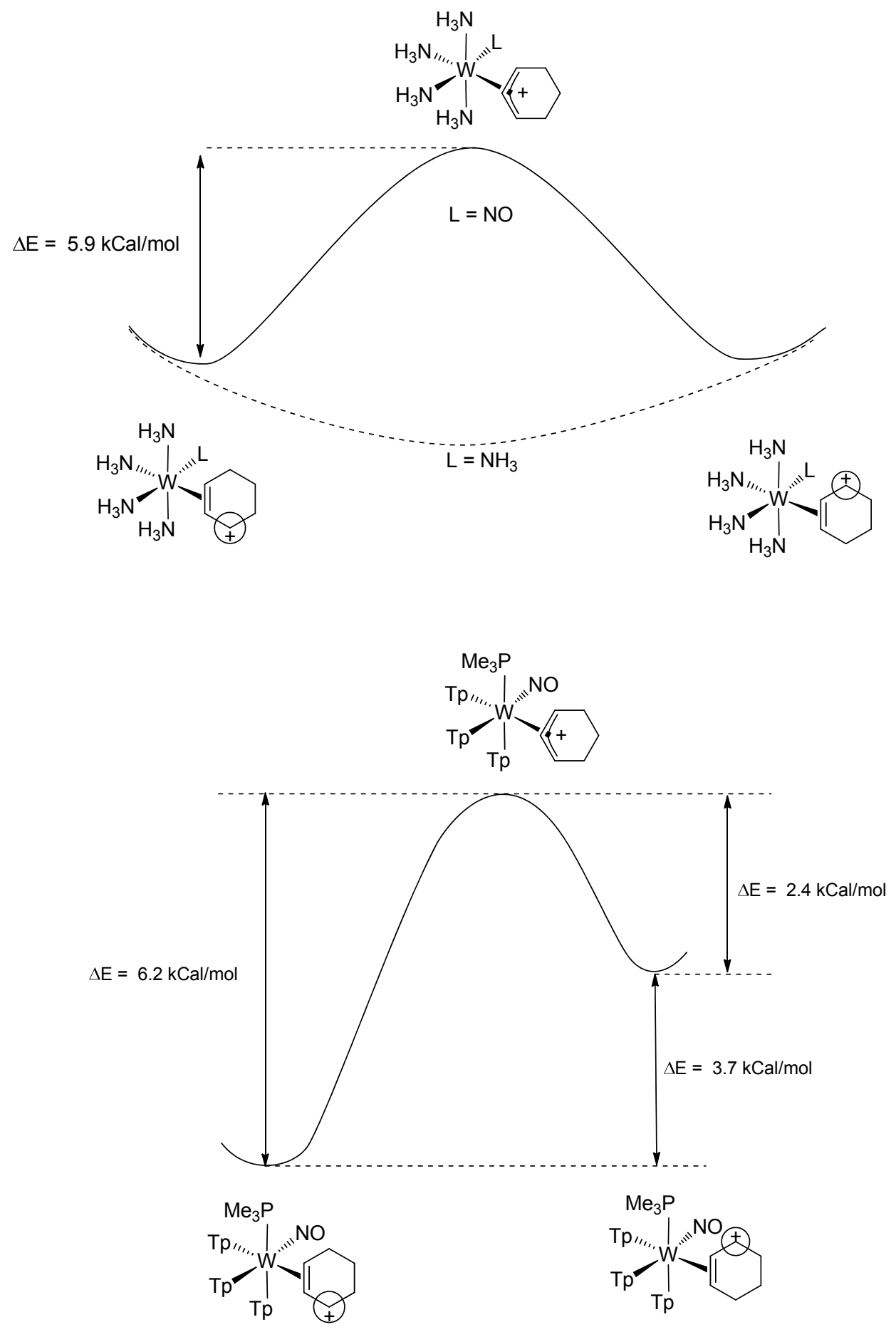
Figure S2 (continued):

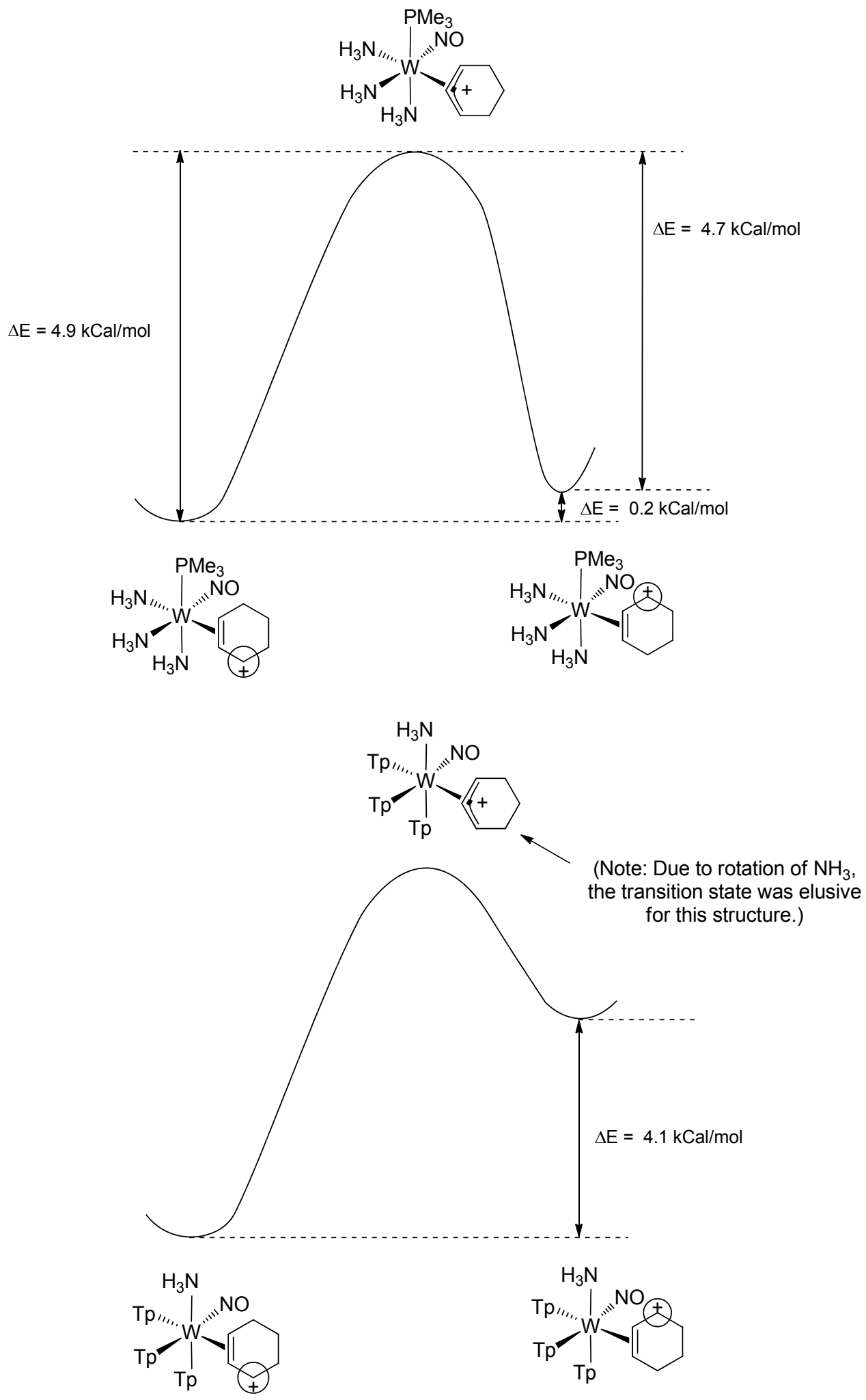




\section{Supplemental Information References:}

(1) Welch, K. D.; Harrison, D. P.; Lis, E. C.; Liu, W.; Salomon, R. J.; Harman, W. D.; Myers, W. H., Organometallics 2007, 26 (10), 2791-2794.

(2) Harrison, D. P.; Sabat, M.; Myers, W. H.; Harman, W. D., J. Am. Chem. Soc. 2010, 132, 17282-17295.

(3) Harrison, D. P.; Zottig, V. E.; Kosturko, G. W.; Welch, K. D.; Sabat, M.; Myers, W. H.; Harman, W. D., Organometallics 2009, 28 (19), 5682-5690.

(4) Lis, E. C.; Delafuente, D. A.; Lin, Y.; Mocella, C. J.; Todd, M. A.; Liu, W.; Sabat, M.; Myers, W. H.; Harman, W. D., Organometallics 2006, 25 (21), 5051-5058.

(5) Salomon, R. J.; Todd, M. A.; Sabat, M.; Myers, W. H.; Harman, W. D., Organometallics 2010, 29 (4), 707-

(6) Todd, M. A.; Sabat, M.; Myers, W. H.; Harman, W. D., Journal of the American Chemical Society 2007, 129 (36), 11010-11011.

(7) Y. Shao; L.F. Molnar; Y. Jung; J. Kussmann; C. Ochsenfeld; S.T. Brown; A.T.B. Gilbert; L.V. Slipchenko; S.V. Levchenko; D.P. O’Neill; R.A. DiStasio Jr.; R.C. Lochan; T. Wang; G.J.O. Beran; N.A. Besley; J.M. Herbert; C.Y. Lin; T. Van Voorhis; S.H. Chien; A. Sodt; R.P. Steele; V.A. Rassolov; P.E. Maslen; P.P. Korambath; R.D. Adamson; B. Austin; J. Baker; E.F.C. Byrd; H. Dachsel; R.J. Doerksen; A. Dreuw; B.D. Dunietz; A.D. Dutoi; T.R. Furlani; S.R. Gwaltney; A. Heyden; S. Hirata; C-P. Hsu; G. Kedziora; R.Z. Khalliulin; P. Klunzinger; A.M. Lee; M.S. Lee; W.Z. Liang; I. Lotan; N. Nair; B. Peters; E.I. Proynov; P.A. Pieniazek; Y.M. Rhee; J. Ritchie; E. Rosta; C.D. Sherrill; A.C. Simmonett; J.E. Subotnik; H.L. Woodcock III; W. Zhang; A.T. Bell; A.K. Chakraborty; D.M. Chipman; F.J. Keil; A.Warshel; W.J. Hehre; H.F. Schaefer; J. Kong; A.I. Krylov; Gill, P. M. W.; Head-Gordon, M., Phys. Chem. Chem. Phys. 2006, 8, 3172.

(8) http://www.gaussian.com/g tech/gv5ref/gv5ref toc.htm.

(9) M. J. Frisch; G. W. Trucks; H. B. Schlegel; G. E. Scuseria; M. A. Robb; J. R. Cheeseman; G. Scalmani; V. Barone; B. Mennucci; G. A. Petersson; H. Nakatsuji; M. Caricato; X. Li; H. P. Hratchian; A. F. Izmaylov; J. Bloino; G. Zheng; J. L. Sonnenberg; M. Hada; M. Ehara; K. Toyota; R. Fukuda; J. Hasegawa; M. Ishida; T. Nakajima; Y. Honda; O. Kitao; H. Nakai; T. Vreven; J. A. Montgomery, J.; J. E. Peralta; F. Ogliaro; M. Bearpark; J. J. Heyd; E. Brothers; K. N. Kudin; V. N. Staroverov; R. Kobayashi; J. Normand; K. Raghavachari; A. Rendell; J. C. Burant; S. S. Iyengar; J. Tomasi; M. Cossi; N. Rega; J. M. Millam; M. Klene; J. E. Knox; J. B. Cross; V. Bakken; C. Adamo; J. Jaramillo; R. Gomperts; R. E. Stratmann; O. Yazyev; A. J. Austin; R. Cammi; C. Pomelli; J. W. Ochterski; R. L. Martin; K. Morokuma; V. G. Zakrzewski; G. A. Voth; P. Salvador; J. J. Dannenberg; S. Dapprich; A. D. Daniels; O. Farkas; J. B. Foresman; J. V. Ortiz; J. Cioslowski; Fox, D. J. Gaussian 09, Revision A.1; Gaussian, Inc., Wallingford CT, 2009. 